FACULTAD DE CIENCIAS

Universidad de Salamanca

\title{
Design and development of a high power high repetition rate ultrashort pulse laser for proton acceleration
}

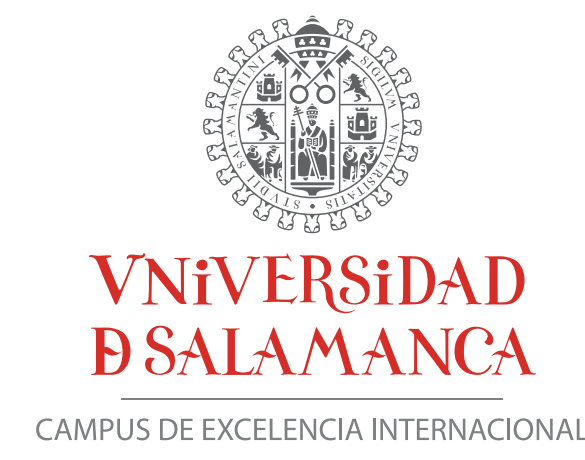

\author{
Tesis Doctoral \\ Roberto Lera Matellanes
}

Directores

Luis Roso

José María Benlloch

Salamanca, Noviembre de 2017 

LUIS ROSO FRANCO, Catedrático del Área de Óptica del departamento de Física Aplicada de la Universidad de Salamanca, CERTIFICA:

Que la presente memoria, "Design and development of a high power high repetition rate ultrashort laser for proton acceleration" / "Diseño y desarrollo de un láser de alta potencia y alta tasa de repetición para la aceleración de protones" ha sido realizada bajo su dirección por el Licenciado Roberto Lera Matellanes y constituye su Tesis Doctoral para optar al grado de Doctor en Física.

En Salamanca, a 28 de Noviembre de 2017 


\section{Agradecimientos}

Todo el trabajo plasmado en esta tesis no habría sido posible sin la aportación de todas y cada una de las personas que me han acompañado durante esta etapa de mi vida, de manera más o menos directa.

En primer lugar, dar las gracias a los dos directores de esta tesis, Luis Roso y José María Benlloch, por su paciencia a la hora de supervisar esta tesis así como por todo el apoyo y la confianza recibidos y por brindarme la oportunidad de comenzar una carrera investigadora.

Me gustaría mostrar mi gratitud a todos los profesores del grupo de óptica de la Universidad de Salamanca por inculcar en mi la pasión por la óptica que tan bien supisteis transmitir. También a la gente del CLPU, que me ha tratado de manera excelente, en especial a Fran, con quien compartí los primeros pasos de mi vida laboral.

Dar las gracias a todo el equipo de Proton Laser que durante todo este tiempo hemos vivido dentro de la sala blanca. A Abel e Isidre, por enseñarme que la electrónica no está tan mal y que los condensadores pueden volar. A Joaquín "el forro" (no sé si se puede calificar de electrónica a alguien que no sabe soldar) gracias por mostrarme todo un vocabulario nuevo tan rico. A Alex, por estos años de convivencia y a Juan, experto en hacer josemarías, así como a Isa y Paula porque he podido aprender mucho con vosotros y hemos podido compartir tantos y tantos momentos dentro y fuera del laboratorio, sin olvidarme de Pablo, un aficionado más a la óptica nuclear. Qué decir de Salomón, que tanto te monta un láser como que te recoge unas naranjas. A Miguel Galán, por demostrarme que uno no puede confiar en los electrónicos. A Delfí por darme la oportunidad de trabajar en este proyecto tan bonito. 
Felipe merece una mención aparte pues a él le debo una buena parte de esta tesis. Confió en mi desde el principio y ha estado pendiente de la realización de este trabajo hasta el final, aparte de haber sido compañero de tantos viajes en los que sufrimos ataques ornitológicos y disfrutamos de suculentos banquetes. Gracias por todo.

Por último, dar las gracias a toda mi familia y amigos porque nada de esto habría sido posible sin vosotros. A mis cuatro padres y a mi hermano Miki, que algún día será ingenierico. No sé si agradecer algo a Ainhoa, puesto que dudo de que haya hecho suficientes méritos como para ser mencionada aquí. Y gracias a Leticia, por cada día, por crecer juntos. 


\title{
List of acronyms
}

\author{
ASE Amplified Spontaneous Emission \\ CPA Chirped Pulse Amplification \\ CW Continuous Wave
}

DASB 3-amino-4-(2-dimethylaminomethylphenylsulfanyl)-benzonitrile

DTBZ Dihydrotetrabenazine

FDG 2-fluoro-2-deoxyglucose

FWHM Full Width Half-Maximum

GDD Group Delay Dispersion

HEM Heat Exchanger Method

KDP Potassium Dihydrogen Phosphate

KTP Potassium Titanyl Phosphate

MOPA Master Oscillator Power Amplifier

OAP Off-Axis Parabola

OPA Optical Parametric Amplifier

OPCPA Optical Parametric Chirped-pulse Amplification

PET Positron Emission Tomography

PMT Photo-Multiplier Tube 
QCW Quasi-Continuous Wave

RCF Radiochromic Film

RF Radio-Frequency

ROC Radius of Curvature

RMS Root Mean Square

SHG Second Harmonic Generation

TOD Third Order Dispersion

TOF Time of Flight

VCSEL Vertical-Cavity Surface-Emitting Laser

VMAT2 Vesicular Monoamine Transporter 2

YAG Yttrium Aluminium Garnet

YLF Yttrium Lithium Fluoride 


\section{Contents}

List of publications xiii

1 Introduction and motivation 1

2 Amplification of pulses 11

2.1 Frantz-Nodvik equation . . . . . . . . . . . . . 13

2.2 Amplification limits . . . . . . . . . . . . . . . . 16

2.3 Deformation of the spatial profile . . . . . . . . . . 18

2.4 Deformation of the temporal profile . . . . . . . . . . . . . 21

2.4 .1 Gain narrowing . . . . . . . . . . . . . . 23

2.4 .2 Contrast . . . . . . . . . . . . . . . . 24

2.5 Non-linear effects . . . . . . . . . . . . . . . . . 26

3 Diode pumping heads $\quad 29$

3.1 Nd-doped materials . . . . . . . . . . . . . . . . . . . . . . . 29

3.1 .1 Nd:YAG . . . . . . . . . . . . . . . . 30

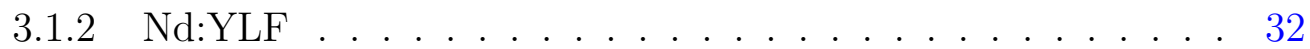

3.2 Diode Pumping heads: design . . . . . . . . . . . . . . . . 33

3.2.1 High power pumping head: the PH5400 . . . . . . . . . . . . 34

3.2 .2 Laser diodes . . . . . . . . . . . . . . . . . . . . . 35

3.2.3 Simulations of pumping distribution . . . . . . . . . . . . 38

3.3 Thermal induced effects . . . . . . . . . . . . . . . . . . . . . . 49

3.3.1 Thermal lensing . . . . . . . . . . . . . . . . . . . 49

3.3.2 Birefringence . . . . . . . . . . . . . . . 52

3.4 Diode Pumping heads: QCW performance . . . . . . . . . . . 53

3.4.1 Theoretical description of QCW performance. . . . . . . . 53 
3.4 .2 Nd:YAG QCW performance . . . . . . . . . . . . . . 59

3.4 .3 Nd:YLF QCW performance . . . . . . . . . . . . 61

4 Development of a nanosecond laser $\quad 65$

4.1 Diode Pumping heads: Q-switching performance . . . . . . . . . 66

4.1 .1 Q-switch theory . . . . . . . . . . . . . . 66

4.1.2 Nd:YAG Q-switching performance . . . . . . . . . . . . . 69

4.1.3 Nd:YLF Q-switching performance . . . . . . . . . . . . 75

4.2 Nanosecond Nd:YAG MOPA . . . . . . . . . . . . . . . . 77

4.3 Nanosecond Nd:YLF MOPA . . . . . . . . . . . . . . . . . . . 79

5 Development of an ultrashort pulse laser $\quad 81$

5.1 Titanium:Sapphire . . . . . . . . . . . . . . . . . . . 82

5.2 CPA laser . . . . . . . . . . . . . . . . . 85

5.2 .1 Femtosecond oscillator . . . . . . . . . . . . 88

$5.2 .2 \quad$ Stretcher . . . . . . . . . . . . . . . . . . . 88

5.2 .3 Regenerative amplifier . . . . . . . . . . . . . . 90

$5.2 .4 \quad$ First multipass amplifier . . . . . . . . . . . . . . . 94

5.2 .5 Second multipass amplifier . . . . . . . . . . . . . 98

5.2 .6 Compressor . . . . . . . . . . . . . . . . . . 103

5.3 Improvement of contrast . . . . . . . . . . . . . . 105

6 Laser acceleration experiments 107

6.1 Proton acceleration mechanisms . . . . . . . . . . . . . . 107

6.2 Experimental setup . . . . . . . . . . . . . . . . . . . 111

6.2 .1 Target holder . . . . . . . . . . . . . . . . . . . 111

6.2.2 Focusing and target positioning . . . . . . . . . . 112

6.3 Proton detection experiments . . . . . . . . . . . . . 115

$6.3 .1 \quad$ CR-39 . . . . . . . . . . . . . . . . . . . . 115

6.3.2 Radiochromic films . . . . . . . . . . . . . . . . . . 116

6.3.3 Scintillator and time-of-flight . . . . . . . . . . . . . 117

6.3.4 Thomson Parabola . . . . . . . . . . . . . . . . . . . . 120

6.4 Experimental results . . . . . . . . . . . . . . . . . . . . 121

6.4.1 Low contrast regime . . . . . . . . . . . . . . . . 122 
6.4.2 High contrast regime . . . . . . . . . . . . . . . 124

6.5 Discussion . . . . . . . . . . . . . . . . . 127

$\begin{array}{ll}\text { Conclusions } & 131\end{array}$

Spanish summary / Resumen en español 135

$\begin{array}{lr}\text { Bibliography } & 155\end{array}$ 


\section{List of publications}

This report is based on the following publications:

1. "Simulations of the gain profile in a diode side-pumped Nd:YAG laser"

R. Lera, F. Valle-Brozas, S. Torres-Peiró, A. Ruiz-de-la-Cruz, M. Galán, P. Bellido, M. Seimetz, JM. Benlloch and L. Roso

Applied Optics 55 (33), 9573-9576 (2016)

2. "Characterization of protons accelerated from a 3 TW table-top laser system" P. Bellido, R. Lera, M. Seimetz, A. Ruiz-de la Cruz, S. Torres-Peirò, M. Galán, P. Mur, I. Sánchez, R. Zaffino, L. Vidal, A. Soriano, S. Sánchez, F. Sánchez, MJ. Rodríguez-Álvarez, JP. Rigla, L. Moliner, A. Iborra, L. Hernández, D Grau-Ruiz, AJ. González, JJ. García-Garrigos, E. Díaz-Caballero, P. Conde, A. Aguilar, L. Roso and JM. Benlloch Journal of Instrumentation 12 (05), T05001 (2017)

3. "Development of a few TW Ti:Sa laser system delivering $100 \mathrm{~mJ}$ pulses at a $100 \mathrm{~Hz}$ repetition rate"

R. Lera, S. Torres-Peiró, P. Bellido, P. Mur, M. Seimetz, JM. Benlloch, L. Roso and A. Ruiz-de-la-Cruz

Submitted 


\section{Chapter 1}

\section{Introduction and motivation}

The invention and development of the laser made a profound impact on science and life in the past century. Since the accomplishment of the first amplified stimulated emission in 1960 by Maiman [1], these devices found their way to our homes (CD-DVD readers), hospitals, military, contributing to several Nobel prizes along the way (Townes, Basov and Prokhorov in 1964, Gabor in 1971, Bloembergen and Schawlow in 1981, Chu, Cohen-Tannoudji and Phillips in 1997, Zewail in 1999). In the present century the impact of lasers is expected to be greater.

During the past decades, the technological progress has pushed the development of increasingly greater laser power and shorter pulse duration. The technique of Q-Switching enabled pulses with a duration in the range of a nanosecond and intensities up to hundreds of MWs. It is based on an abrupt change of the so-called quality factor of the cavity while the energy builds up in the laser medium. When the losses are suddenly removed the accumulated energy is released in a short span of time, resulting in high intensities. There have been several methods devised for Q-switching which are divided into active, when there is external control over the losses of the cavity (Pockels cells, acousto-optic), or passive, where a self-regulated process creates the modulation of the quality factor.

Shorter pulses were achieved with the advent of the mode-locking technique [2]. It takes advantage of the fact that a cavity has a discrete collection of resonant modes separated in frequency; these modes can be amplified if they fall within the bandwidth of the amplification medium. This comb of modes will generally produce an apparently random signal, however, if the modes have some correlation 


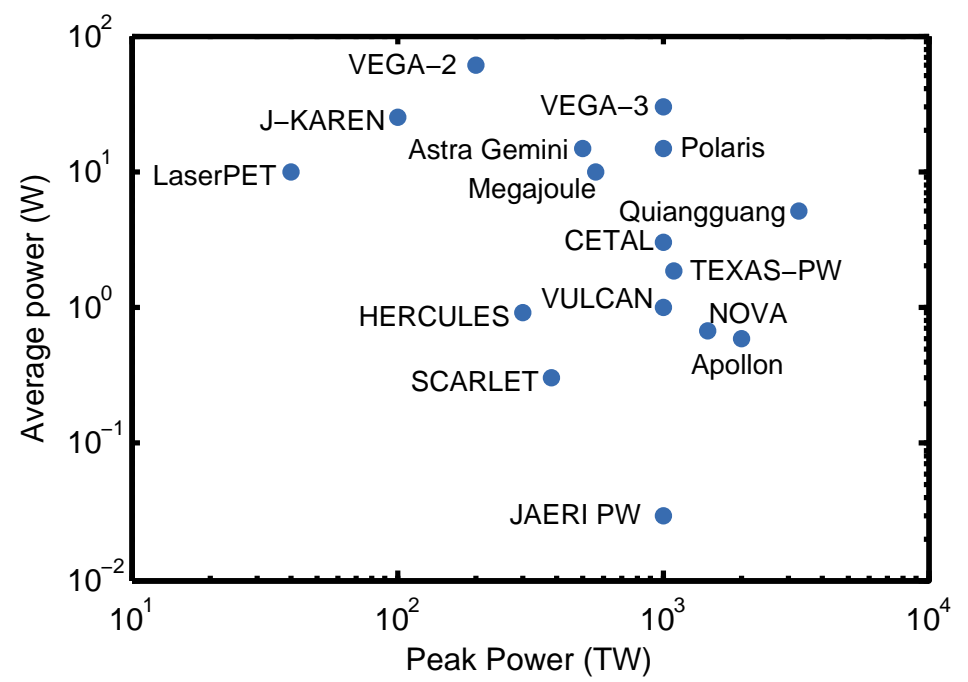

Figure 1.1: Plot of a selection of representative ultraintense lasers operative around the world. LaserPET (Spain), J-KAREN (Japan), VEGA2-3 (Spain), Astra Gemini (UK), Polaris (Germany), Megajoule (France), Quiangguang (China), CETAL (Romania), TEXAS-PW (USA), VULCAN (UK), HERCULES (USA), Apollon (France), NOVA (USA), SCARLET (USA) and JAERI PW (Japan).

between their absolute phase a train of short pulses will be produced, the wider the bandwidth, the shorter the pulse. With mode-locking, pulses of picosecond and femtosecond duration [3] and several GWs of peak power have been produced. In order to achieve this lock of phases, there are several techniques that use passive effects, external injection modulation or non-linear effects, like Kerr lens mode-locking [4]. Mode locking and post compression techniques have enabled the generation of pulses close to an optical cycle [5].

The introduction of ultrashort pulses represented a challenge to laser technology because amplification of such short pulses caused damage to all materials since they broke down under high peak power. The solution to this issue was the introduction of Chirped Pulse Amplification (CPA). Developed in 1985 [6], the idea behind the technique is to subject the laser pulse to a stage of temporal stretching, one or several stages of amplification, and a stage of temporal re-compression.

This technique allowed the peak power of lasers to grow from Gigawatts to Terawatts and beyond. As of the writing of this thesis, Pettawatt laser systems are starting to become a common instrument in research laboratories all over the world, some of them represented in figure 1.1. Laser light can be focused onto an area of 
a few square microns which combined with the peak power that can be achieved in CPA systems, generates intensities at the focus of the order of $10^{21} \mathrm{~W} / \mathrm{cm}^{2}$. The ability to reach such high intensities opens up many applications in the field of high energy physics. One of the most promising applications of lasers is their potential use as novel sources of accelerated particles or extreme light coherent beams. At these extremes levels of intensity, when an ultra-intense laser shines on a material, atoms are ionized and a plasma is formed. In laser-plasma interaction, electrons are accelerated thanks to the longitudinal electric field generated by the ponderomotive force of an ultra-short and ultra-intense laser. This rapidly oscillating field is not enough to accelerate protons nor ions, but the separation of charge produced by the plasma dynamics creates a capacitor-like electric field that can accelerate protons and ions.

The characteristic features of laser-driven particle beams compared with conventional radio-frequency $(\mathrm{RF})$ accelerators are that the effective source size of ion emission is extremely small, in the order of microns, as well as the bunch temporal width, in the order of picoseconds. However, the most remarkable feature of the laser acceleration process is that the gradients are extraordinarily high of the order of $\mathrm{MeV}$ per micron, compared to $\mathrm{MeV}$ per meter provided by conventional accelerators [7]. This high acceleration gradient promises to diminish greatly the size (and thus the cost) of current acceleration facilities. As an example, $1 \mathrm{GeV}$ electrons were generated in less than $3 \mathrm{~cm}$ [8], whereas the same electron energy requires diameters up to $100 \mathrm{~m}$ in conventional accelerators such as synchrotrons.

However, accelerated particle beams generated with lasers lack some of the features provided by RF accelerators, such as spectral and angular control of the beam; although transversal and longitudinal electric fields may be present in the laser plasma interaction that focus the beam, such a passive method usually falls behind more active beam shaping devices like quadrupole magnets, hard to implement but fit nicely into the RF amplification chain. Also, conversion efficiency from laser energy to the ion beam, emittance, current, as well as stability of the acceleration parameters are some weaknesses of laser acceleration with respect to RF acceleration. Moreover, most laser systems are not able to reach high enough repetition rates, less than $10 \mathrm{~Hz}$, in contrast to $\mathrm{MHz}$ repetition rates in $\mathrm{RF}$ accelerators. 
In spite of these drawbacks, the advantages of laser particle acceleration position it as an attractive alternative for a future substitute of RF accelerators in certain applications. One of them is ion beam therapy, the treatment of tumours with proton or ion beams. This therapy offers advantages with respect to conventional photon or electron radiotherapy thanks to the properties of a hadron beam interacting with matter. When a proton enters a material it slows down losing energy quasiconstantly throughout its path until it is slow enough and is absorbed completely in a very short length. This leads to a well defined and localized peak of energy deposition known as the Bragg peak; the position of the Bragg peak depends on the energy and ion species as well as the characteristics of the traversed material, therefore it is possible to control the depth inside the body of the Bragg peak. This therapy is specially indicated for the treatment of tumours that are located in the close vicinity of a vital organ. The dose deposited by an ion beam would minimize the dose received by that organ and maximize the dose at the tumorous mass. The most frequently used ions in therapy are protons and carbon ions. Carbon ions are favoured because of their higher mass, that results in an increase of dose efficiency by up to a factor of 3 when compared to protons or conventional radiotherapy.

Currently, as of April 2017 there are about 62 proton and 11 carbon therapy facilities worldwide in operation, compared to 42 and 8 respectively in 2015 [9]. This relatively low number is primarily caused by the huge cost and size of the infrastructure needed to house a RF accelerator that produces $200 \mathrm{MeV}$ protons or $1 \mathrm{GeV}$ carbon ions, as well as the engineering needed to guide an ion beam from the accelerator to the position of the patient, using heavy magnets integrated inside a gantry that must be able to rotate around the patient. The substitution of one of these RF accelerators for a laser-based accelerator would reduce significantly the required area and the proton or ion source could be located very close to the patient. The heavy magnets inside the gantry could be substituted by mirrors that can be rotated around the patient and would pose no radiation hazard to the patient or operators, reducing the shielding requirements to a minimum. Furthermore, the protons are grouped in bunches of similar duration to that of the laser pulse which may reach tens of femtoseconds. This feature might have higher radio-biological effectiveness than conventional RF-produced beams [10].

However, there is another medical application of lasers that requires less strin- 


\begin{tabular}{llll}
\hline Radioisotope & Half-life $(\min )$ & Production reaction & Labelled molecule \\
\hline${ }^{11} \mathrm{C}$ & 20.3 & ${ }^{11} \mathrm{~B}(\mathrm{p}, \mathrm{n}){ }^{11} \mathrm{C}$ & DASB, DTBZ \\
${ }^{13} \mathrm{~N}$ & 10.0 & ${ }^{13} \mathrm{C}(\mathrm{p}, \mathrm{n}){ }^{13} \mathrm{~N}$ & Ammonia \\
${ }^{15} \mathrm{O}$ & 2.0 & ${ }^{15} \mathrm{~N}(\mathrm{p}, \mathrm{n}){ }^{15} \mathrm{O}$ & Water \\
${ }^{18} \mathrm{~F}$ & 109.8 & ${ }^{18} \mathrm{O}(\mathrm{p}, \mathrm{n}){ }^{18} \mathrm{~F}$ & FDG \\
\hline
\end{tabular}

Table 1.1: Half-life, nuclear reaction and main labelled molecules of the most commonly used radioisotopes in PET imaging.

gent parameters and may be more feasible in the near future: the production of radionuclides for PET diagnostic. PET is an imaging technique that stands for Positron Emission Tomography and requires the production of short-lived positron emitting isotopes that must be combined with some acceptor organic molecule, which is then called a radiopharmaceutical or radiotracer. A PET scanner consists of a ring of scintillating detectors that gather the gamma ray pairs produced by electron-positron annihilations inside the body. The exact position of the radiotracer is traced by coincidence, the simultaneous detection of the gamma ray photons generated in the annihilation events.

The most common positron-emitting isotopes are ${ }^{11} \mathrm{C},{ }^{13} \mathrm{~N},{ }^{15} \mathrm{O}$ and especially ${ }^{18} \mathrm{~F}$; their properties are summarized in table 1.1. Carbon-11 can label any compound containing carbon, which is vital in biology, so it has the potential to be a very powerful diagnostic and research tool, especially in neuroscience. DASB is a compound that binds to the serotonin transporter, and so it can be used to study several mental health disorders such as depression [11]; DTBZ is a compound with affinity for VMAT2, a protein considered as a biomarker for Parkinson's disease [12]; Raclopride is a compound that acts as a selective antagonist on $\mathrm{D}_{2}$ dopamine receptors, which can be useful for the diagnosis of movement disorders [13]. Due to its extremely short lifetime, Oxygen-15 is the least used radioisotope due to technological challenges that involve its distribution to a patient. It can label simple molecules such as water; its distribution and clearance are completely dependent on the rate of blood flow, so it has been used to measure cerebral blood flow and myocardium perfusion [14]. Ammonia $\left(\mathrm{NH}_{3}\right)$, labelled with nitrogen-13, is administered intravenously and is delivered to several organs, such as the liver, 
kidneys, brain and myocardium [15]. It is retained in the cells following conversion to glutamine by glutamine synthetase, an enzyme that plays a key role in cancer cell proliferation [16]. The most used radiotracer by far is 2-fluoro-2-deoxyglucose, commonly known as FDG, labelled with ${ }^{18} \mathrm{~F}$. The importance of this molecule lies in that it is a glucose analogue, and as such it can be incorporated into the glucose metabolism, therefore it is crucial to study cerebral and myocardial glucose intake or diseases with an abnormal metabolic rate such as most kinds of tumours [17], including breast, colon, pancreatic, oesophageal, head and neck, lung cancer, lymphoma and melanoma [18]. The prevalence of this compound in the PET studies is due to its economic advantage and the long lifetime of the ${ }^{18} \mathrm{~F}$, which allows it to be transported to PET scanning centres as far as 3 hours away by road.

The major obstacle in the development of PET research and diagnostics resides in the manufacturing of the radiotracers. The labelling radioisotopes are the final products of nuclear reactions triggered by protons, i.e $(\mathrm{p}, \mathrm{n})$ or $(\mathrm{p}, \alpha)$. Cyclotron facilities are currently in charge of the production of the radioisotopes that are then distributed to hospitals or PET scanning facilities. However cyclotrons have some drawbacks that make them less than ideal for their installation in a hospital or clinic. These facilities have a big footprint (more than $100 \mathrm{~m}^{2}$ ) and require additional space for other critical components such as the control room and power supply. Additionally, these machines require strict radiation shielding not only from Bremsstrahlung radiation but especially from neutrons generated in the target area, which translates into thick concrete walls and additional measures such as radiation labyrinths. Maintenance is complicated in these machines, since they can weight up to 40 tons. Another disadvantage of cyclotrons is their lack of versatility: the properties of the ion species they can successfully accelerate are fixed.

Strikingly, many of these obstacles can be circumvented substituting the cyclotron with a laser accelerator. The installation of a cyclotron in a hospital with PET scanning capabilities would require a huge investment, both in terms of space and money. That is the reason why FDG has overshadowed all other radiotracers, sadly relegating the others to research or use only in facilities where a cyclotron is adjacent. Recent advances in technology promise that table-top lasers capable of producing clinical doses of PET radioisotopes would be less space-restrictive, filling a volume smaller than conventional cyclotrons and minimizing the radiation 


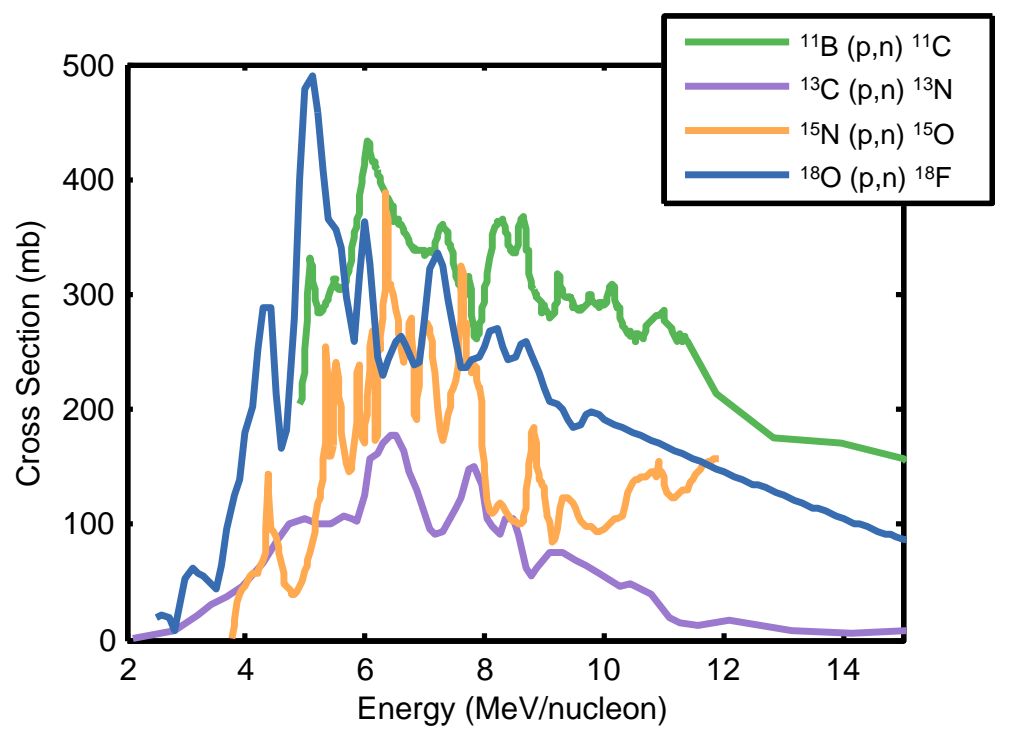

Figure 1.2: Cross sections of the proton-triggered nuclear reactions where the most used radioisotopes in PET tracers are generated. Data gathered from the EXFOR library [19].

shielding requirements to a very confined area, where the protons and radiotracers are generated. Laser accelerators also shine in their versatility; the same laser could deliver various ion species without the modification of any parameter of the laser, just by changing the primary target, which can be a solid foil or a gas. The chemistry required to bond the isotope to the organic molecule is identical to the one currently used in cyclotron-produced radiotracers; the only difference would be the dosage: cyclotrons currently produce batches of radioisotopes to distribute to several patients but a laser would produce a single dose for a single patient. The localized manufacture of radioisotopes would boost the research and implementation not only of FDG but also other less used radiotracers, that are not currently practical to use because of their short lifetime.

The cross sections of the principal nuclear reactions that produce the radioisotopes used in PET (figure 1.2) have a maximum for proton energies between 5-8 $\mathrm{MeV}$. Laser accelerated protons have already reached these energies and beyond using table-top lasers [20]. Furthermore, proof-of-principle experiments of the production of PET radioisotopes have already been realized [21-23]. However, as of the writing of this thesis, state-of-the-art lasers cannot produce a human clinical dose of a PET radioisotope. It has been estimated that reaching radioisotope 
production capabilities requires moderate laser peak power, around tens of TW, an intensity of $10^{20} \mathrm{~W} / \mathrm{cm}^{2}$ but average power in excess of $500 \mathrm{~W}[24,25]$.

This thesis pretends to be a step forward towards the realization of a laser capable of fulfilling the requirements for radioisotope production, advancing the frontiers of laser technology. Currently there are several viable laser materials for ultraintense operation: Ti:Sa, Yb-ion, Nd:glass, etc. and other techniques that might be useful for that purpose such as OPCPA [26]. Amongst them all, Titanium:Sapphire (Ti:Sa) stands out due to its broad bandwidth, the hardiness of its host crystal and the maturity of the technology. The absorption bandwidth of this material extends through most of the visible spectrum, but currently there is a lack of efficient pump sources in that zone of the spectrum. Presently, the most successful way of pumping a Ti:Sa laser is the second harmonic line of a Nd-doped laser. The majority of the most powerful lasers in the world in figure 1.1 are based on Ti:Sa. These solid-state lasers are limited to $10 \mathrm{~Hz}$ repetition rate because they are pumped by flashlamps, the main reason for the lack of pump lasers with repetition rate higher than $100 \mathrm{~Hz}$.

Hence, new advancements in solid-state pumping technology must be developed for the new generation of high average power ultraintense lasers. Laser diodes were first used as pump sources a short time after the invention of the laser [27], but technological obstacles did not make them commercially viable until the nineties. They present a series of characteristics that make them excellent candidates as a high repetition rate pumping source [28]: high plug efficiency, very long lifetime, compactness and monocromaticity; the main disadvantages of laser diodes are their high cost and low output power compared to flashlamps, issues that are continuously improving as technology advances.

This thesis reports the construction of a TW-class high average power Titanium:Sapphire laser at $100 \mathrm{~Hz}$ repetition rate powered by diode-pumped Nd-doped lasers. In order to develop the lasers that would pump the Ti:Sa laser, a collaboration with the company Monocrom S.L ${ }^{1}$ resulted in the design and construction of diode side-pumped heads, called PH5400, that serve as a building block for the development of high average power pump lasers suited to pump a Ti:Sa crystal. In order to prove the concept, a particle acceleration experiment was carried out in

\footnotetext{
${ }^{1}$ Monocrom S.L, Spain, monocrom.com
} 
order to validate the completed TW laser as a table top proton source.

The second chapter introduces the theory behind pulse amplification and some challenges associated to ultrashort pulse amplification, specially gain narrowing. The main goal of this chapter is to develop a series of simulation tools of the amplification process, according to the theory of Frantz-Nodvik, in order to be employed during the development of the laser. It is valid for the amplification of both Nd:YAG nanosecond pulses and Ti:Sa stretched pulses.

The third chapter introduces the PH5400, a diode-powered pumping head that can reach up to $1 \mathrm{kHz}$ repetition rate. An analysis of the diode arrangement inside the pumping chamber is made, involving simulations of the absorbed power distribution. The performance of these laser modules when used with Nd:YAG and Nd:YLF is investigated in pulse mode and issues that appear at high power operation are discussed.

The fourth chapter reviews the concept and theory of Q-switch and how this technique can be applied to obtain nanosecond pulses from Nd:YAG and Nd:YLF. This chapter also covers the development of a high average power nanosecond laser, built with the PH5400 pumping heads, suitable for pumping the Ti:Sa CPA chain.

The fifth chapter reports on the construction and performance of the ultraintense Ti:Sa laser. First, the laser system is briefly described and each stage of the CPA chain is detailed, supported by the simulations of amplification and spectrum developed in chapter 2 .

In the sixth and last chapter, the ultraintense laser developed in this thesis is used as a source of accelerated protons. The experimental setup and the nuclear detectors used are reviewed. Experimental results show the dependence of the proton beam energy as a function of target material and thickness during a low contrast and a high contrast campaign. The results are compared to that of other proton acceleration experiments published in literature. 


\section{Chapter 2}

\section{Amplification of pulses}

The production of energetic short pulses that support broad spectra is one of the current hot topics in laser technology. The amplification of light requires a beam and a gain medium and relies in one of the fundamental interactions of light and matter: stimulated emission of light. The gain medium can take multitude of different forms and materials that provide singular properties to the laser emission and research in this field is constantly generating novel materials.

The formalism of amplification is well-known and can be pretty simple. The incident laser is considered as a bunch of $n$ photons. The incident flux is defined as $\phi_{i}=n / A$, while the active medium can be described as a volume $A \cdot L$, where $A$ is the total area and $L$ the total length of the active medium. The change in the photon flux is proportional to $N$, the number of excited atoms and the probability that one of these atoms is de-excited, parametrized by the emission cross section $\sigma$, per unit length $z$ of the gain medium:

$$
d \phi=\phi \sigma N d z
$$

Assuming that the atoms are uniformly distributed along the active medium, equation 2.1 can be integrated to obtain the flux of photons after traversing the active medium:

$$
\phi_{f}=\phi_{i} \exp (\sigma N L)
$$

This is the so-called linear regime of amplification, because the output flux is 


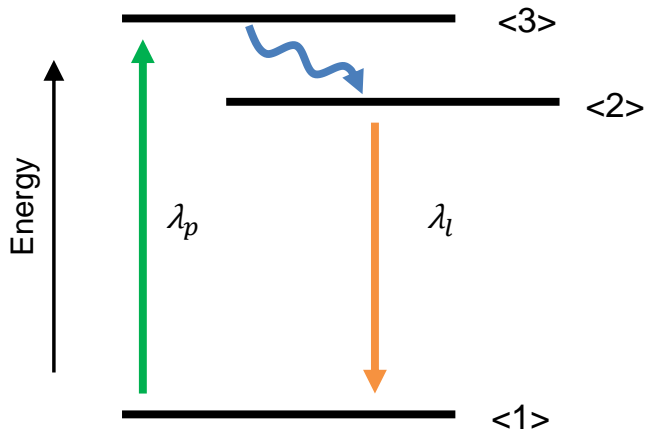

(a)

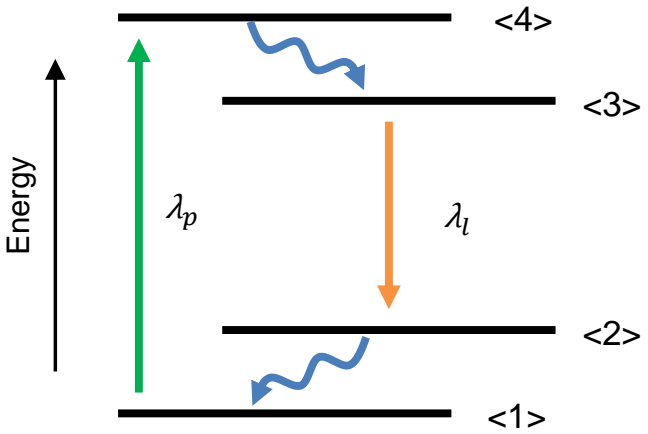

(b)

Figure 2.1: a) Scheme of a three level system. b) Scheme of a four level system.

proportional to the input through a constant that depends only on parameters of the active medium. The term $\sigma N$ is usually called small-signal gain represented as $g$ :

$$
g=\sigma N
$$

The total gain, defined as the ratio of the output and input flux is:

$$
G=\exp (g L)
$$

The parameter $N$ represents the population inversion between the two atomic levels which constitute the laser transition, $N=N_{2}-N_{1}$. Equation 2.2 shows that there is amplification only if $N_{2}>N_{1}$ while if $N_{2}<N_{1}$ the input flux is attenuated. However not all atomic systems can support population inversion. For example, the simplest possible scheme, a two level system, cannot have inversion of population when in thermal equilibrium, because the population of each energy level is ruled by the Boltzmann distribution, which establishes that the occupation diminishes exponentially with the energy associated to the level, so necessarily the population of the upper laser level must be lower than that of the fundamental state, $N_{2}<N_{1}$.

More complex systems like three and four level systems do have the properties needed to support population inversion. In a three level system (figure 2.1(a)) the pump excites the excites the fundamental level to an excited state that decays 
quickly to another level, which is de-excited to the fundamental level emitting a photon. This scheme requires high pump power to achieve inversion of population. $\mathrm{Cr}^{3+}$ ion based lasers are good representatives of this scheme.

In a four level system (figure 2.1(b)) light is pumped from the fundamental level to an excited state which rapidly decays as non-radiative radiation to another excited level. The emission of a photon de-excites this state to the lower laser level which rapidly decays to the fundamental state. Thus the lower laser level will be almost empty at all times which provides inversion of population even with low pump intensity. Nd:YAG, Nd:YLF and Ti:Sa, the materials employed in this thesis can all be well described as a four level system.

\subsection{Frantz-Nodvik equation}

Note that we could perform the integration of equation 2.1 because we made the assumption that the population inversion is independent of the incident flux. However, when a laser has a high enough flux of photons it will be able to modify the balance of the populations, and eventually will enter a regime of saturation when the upper state population is depleted.

A general description of amplification can be deduced using the rate equations. We will assume that the active medium has an initial population inversion $N_{0}$ and that the event of amplification is instantaneous compared to the pumping rate and the spontaneous emission lifetime. We can write the rate of population inversion as:

$$
\frac{d N}{d t}=\frac{\sigma}{h \nu} N I
$$

This equation can be integrated with respect to time:

$$
\left.N(z, t)=N(z, t=-\infty) \exp \left(-\frac{\sigma}{h \nu} \int_{-\infty}^{t} I\left(z, t^{\prime}\right)\right) d t^{\prime}\right)
$$

where $N(z, t=-\infty)$ represents population inversion at the position $\mathrm{z}$ before the pulse arrives, that is, $N_{0}$. And the evolution of intensity

$$
\frac{\partial I}{\partial z}-\frac{1}{c} \frac{\partial I}{\partial t}=\sigma N I-\alpha I
$$


where $\alpha$ represents absorption losses in the amplifier. This equation can be integrated in time:

$$
\int_{-\infty}^{\infty}\left(\frac{\partial I}{\partial z}-\frac{1}{c} \frac{\partial I}{\partial t}\right) d t=\int_{-\infty}^{\infty}(\sigma N-\alpha) I d t
$$

The equation can be simplified as the pulse vanishes asymptotically, $\int_{-\infty}^{\infty} \frac{\partial I}{\partial t}=$ $I(z, t=\infty)-I(z, t=-\infty)=0$. We will define a new magnitude called fluence $F$ as:

$$
F(z)=\int_{-\infty}^{\infty} I(z, t) d t
$$

Introducing equations 2.6 and 2.9 into 2.8 we get,

$$
\frac{d F(z)}{d z}=\sigma N_{0}(z) \int_{-\infty}^{\infty} I(z, t) \exp \left(-\frac{\sigma}{h \nu} \int_{-\infty}^{t} I\left(z, t^{\prime}\right) d t^{\prime}\right) d t-\alpha F(z)
$$

Which can be written as,

$$
\frac{d F(z)}{d z}=h \nu N_{0}(z)\left(1-\exp \left(-\frac{\sigma}{h \nu} F(z)\right)\right)-\alpha F(z)
$$

We will define a new magnitude as saturation fluence,

$$
F_{\text {sat }}=\frac{h \nu}{\sigma}
$$

Recalling the definition of small signal gain (equation 2.3)and introducing equation 2.12 into 2.11 ,

$$
\frac{d F}{d z}=g F_{\text {sat }}\left(1-\exp \left(F / F_{\text {sat }}\right)\right)-\alpha F
$$

Equation 2.13 can be solved numerically but it is only possible to obtain an analytical solution if $\alpha=0$. In that case, it can be expressed as:

$$
F(z)=F_{\text {sat }} \ln \left(1+\exp (g z)\left(\exp \left(\frac{F(0)}{F_{\text {sat }}}\right)-1\right)\right)
$$

where $F(0)=F_{\text {in }}$ is the fluence of the input beam; when $z=L$ the laser has completed its passage through the amplifier, hence $F(z=L)=F_{\text {out }}$ is the output fluence. This equation is known as the Frantz-Nodvik equation [29]. We can 
reproduce equation 2.2 in the limit $F_{\text {in }} \ll F_{\text {sat }}$. Expanding in a Taylor series $\exp \left(F_{\text {in }} / F_{\text {sat }}\right) \approx 1+F_{\text {in }} / F_{\text {sat }}$ and substituting in equation 2.14 ,

$$
F_{\text {out }}=F_{\text {sat }} \ln \left(1+\frac{F_{\text {in }}}{F_{\text {sat }}} \exp (g L)\right)
$$

Again, making the approximation $\ln (1+x) \approx x$ when $x \ll 1$,

$$
F_{\text {out }}=F_{\text {in }} \exp (g L)
$$

Which is equivalent to equation 2.2. The other regime, called saturation regime is defined when the input fluence is comparable or greater than the saturation fluence: $\exp \left(F_{\text {in }} / F_{\text {sat }}\right) \gg 1$. Introducing this approximation in the Frantz-Nodvik equation (2.14):

$$
F_{\text {out }}=F_{\text {sat }}\left(g L+\frac{F_{\text {in }}}{F_{\text {sat }}}\right)=F_{\text {in }}+F_{\text {sat }} g L
$$

In this case, the output fluence is no longer proportional to the input fluence. The factor $F_{\text {sat }} g L$ represents the maximum energy density extractable from the active medium. From now on, we shall call this factor $F_{\text {sto }}$. In the saturation regime, gain takes the form:

$$
G=\frac{F_{\text {out }}}{F_{\text {in }}}=1+\frac{F_{\text {sat }} g L}{F_{\text {in }}} \approx 1
$$

Therefore, it is not possible to achieve simultaneously high gain and high extraction efficiency in an amplifier.

In a single-pass amplifier, the Frantz-Nodvik equation can finally be written as

$$
F_{\text {out }}=F_{\text {sat }} \ln \left(1+\exp \left(\frac{F_{\text {sto }}}{F_{\text {sat }}}\right)\left(\exp \left(\frac{F_{\text {in }}}{F_{\text {sat }}}\right)-1\right)\right)
$$

An important consequence of this equation is that the process of amplification is not influenced by the longitudinal distribution of stored energy inside the active medium. In order to prove it, the amplifier is divided in $M$ portions in z, each having its own stored fluence $F_{\text {sto }}^{i}$. Equation 2.19 is applied at each longitudinal slice: 


$$
F_{\text {out }}^{i}=F_{\text {sat }} \ln \left(1+\exp \left(\frac{F_{\text {sto }}^{i}}{F_{\text {sat }}}\right)\left(\exp \left(\frac{F_{\text {in }}^{i}}{F_{\text {sat }}}\right)-1\right)\right)
$$

Assuming no losses within the active medium, the output fluence of a slice is the input fluence of the next: $F_{i n}^{i+1}=F_{\text {out }}^{i}$. Operating in equation 2.20 the output fluence of the last slice $M$ can be expressed as:

$$
F_{\text {out }}^{M}=F_{\text {sat }} \ln \left(1+\exp \left(\sum_{i=1}^{M} \frac{F_{\text {sto }}^{i}}{F_{\text {sat }}}\right)\left(\exp \left(\frac{F_{\text {in }}^{1}}{F_{\text {sat }}}\right)-1\right)\right)
$$

The term $\sum_{i=1}^{M} \frac{F_{s t o}^{i}}{F_{s a t}}$ represents the sum of the stored fluences in each slice, which is equal to the total stored fluence in the active medium. Therefore, we can finally write:

$$
F_{\text {out }}^{M}=F_{\text {sat }} \ln \left(1+\exp \left(\frac{F_{\text {sto }}}{F_{\text {sat }}}\right)\left(\exp \left(\frac{F_{\text {in }}^{1}}{F_{\text {sat }}}\right)-1\right)\right)
$$

It can be concluded from equation 2.22 , that the amplification process is independent of the longitudinal energy distribution within the active medium. However, this should not downplay the importance of the pump distribution, since it influences other parameters such as temperature gradients or mechanical stresses inside the active medium.

\section{$2.2 \quad$ Amplification limits}

It is not always possible to fulfil the conditions to meet a regime of maximum efficiency of amplification, as stored fluence and output fluence are subjected to certain constraints that may limit the performance of the amplifier.

The stored energy in an amplifier was defined as the total energy available for amplification, which is determined by the number of excited atoms in the active medium. Once every atom in the active medium is excited, absorption saturates and the medium becomes transparent to the pump energy, hence the volume and the doping concentration of laser medium dictates a hard cap for the maximum energy storable. Doping concentration cannot be arbitrarily large, since it can affect the integrity of the crystalline structures of the medium. The quality of the laser medium also influences the performance of an amplifier as the presence of 
impurities, that absorb the laser light and contribute to an increase the thermal load, while scattering centres increase diffraction losses that diminish the output fluence.

The power and repetition rate of the pump laser can also limit the performance of an amplifier. Non-radiative transition between levels convert a portion of the pump energy into heat, a percentage that can be $25 \%$ for Nd:YAG and $40 \%$ for Ti:Sa. This effect creates high thermal loads and mechanical stresses that either damage the crystal or limit the amplification because of very powerful thermal lenses or high depolarization. These thermal induced effects will be reviewed in chapter 3 .

The fluorescence lifetime of the active medium limits the amount of energy that is stored with continuous pumping. When a medium is pumped, it also releases a certain amount proportional to the power it receives via spontaneous emission which leads to a stationary behaviour when the pumping time has surpassed the fluorescence lifetime of the material; all the additional energy pumped into the medium is either released as photons or contributes to the heating of active the medium. This is the reason why lasers that have a very short fluorescence lifetime, such as Ti:Sa, must be pumped using nanosecond lasers. The repetition rate of a pulsed laser can also limit the available stored energy in a continuously pumped laser if its period is less than the fluorescence lifetime.

Damage in the crystal can also appear for high optical fluences in the amplifier. One of the most limiting factors is damage in the faces of the active medium. The damage threshold in typical polished raw materials like YAG and sapphire is $>100 \mathrm{~J} / \mathrm{cm}^{2}$ for nanosecond pulses $[30,31]$ and scales non-linearly for lower pulse duration [32]. However, for laser operation an anti-reflection coating is often applied to the faces of these materials and the damage threshold falls to $10 \mathrm{~J} / \mathrm{cm}^{2}$, for $10 \mathrm{~ns}$ pulses at $1064 \mathrm{~nm}$ [33], which is the state of the art for most manufacturers. Damage threshold is a value that decreases linearly with wavelength and non-linearly with pulse duration. 


\subsection{Deformation of the spatial profile}

The Frantz-Nodvik equation was derived under the assumption that the transversal components of the input beam and gain were uniform throughout the entire surface of the active medium. In a real amplifier, this assumption is often incorrect and the spatial profile of either the input beam or the pump beam are far from uniform, which can affect the amplification process. The Frantz-Nodvik equation can be modified to take into account the effects of the transversal dimensions.

Without any loss of generality, we can extend equation 2.19 to the transversal dimensions $\mathrm{x}, \mathrm{y}$. In this new formalism, fluence is a local magnitude and each part of the beam experiences a different gain based on the stored fluence distribution and the local input fluence:

$$
F_{\text {out }}(x, y)=F_{\text {sat }} \ln \left(1+\exp \left(\frac{F_{\text {sto }}(x, y)}{F_{\text {sat }}}\right)\left(\exp \left(\frac{F_{\text {in }}(x, y)}{F_{\text {sat }}}\right)-1\right)\right)
$$

This model considers only geometrical propagation (diffraction effects, for example, are neglected) but it is a reasonable approach for the present study. This equation was solved numerically using a Matlab script that treated the active medium as a volume that was divided into $N_{x} \cdot N_{y}$ nodes. The initial conditions are the stored fluence distribution inside the active medium and the input fluence profiles. Finally, equation 2.23 was solved for each node separately. The output energy per pulse is just the integral over the surface of the fluence:

$$
E_{\text {out }}=\iint F_{\text {out }}(x, y) d x d y
$$

The fact that fluence is a local magnitude has an important consequence: the regime of amplification can be different in several parts of the beam; the parts of the beam where the fluence is high may experience saturation while the parts where the fluence is low experience linear amplification. As a result, the performance of an amplifier depends on the shape of the input beam as well as the distribution of gain inside the active medium, while the output beam profile will also be dependent on the regime of amplification and may suffer alterations. In order to study the influence of amplification in the shape of the output beam we performed some 
simulations where a gaussian beam is amplified in different gain profile scenarios. The results can be observed in figure 2.2 .

If the input fluence is low, the regime is linear so the gain only depends on the profile of the stored fluence. If the pump profile is constant, flat-top like, such as in figure 2.2(a), the output profile is not modified because every part of the beam experiences the same gain and the output profile remains gaussian shaped. However, if the stored fluence profile is gaussian-shaped, as in figure 2.2(c), gain is no longer homogeneous and in this case is higher around the centre. Therefore, the centre part of the input beam experiences higher gain than the outer parts of the beam. The principal consequence is a decrease in the size of the beam; the magnitude of this effect is hard to quantify but must be taken into account in order to avoid optical damage related problems during amplification.

On the other hand, if the input fluence and the gain are high, the amplification process enters the saturated regime. In the case of a gaussian shaped input and uniform gain as in figure 2.2(b), the effect of saturation is more prominent in the centre of the beam where the fluence is higher. As the gain is now higher in the less saturated parts, the beam experiences an increase in size while the peak of the gaussian is flattened. A different behaviour can be observed in figure 2.2(d) for the case of a gaussian-shaped gain. The output beam profile has barely been modified and fits perfectly with a gaussian shape. We can conclude that the effect of amplification in the saturation regime tends to modify the size and shape of the input beam to resemble the profile of the gain distribution. Furthermore, in order to preserve a gaussian beam during amplification, the gain distribution must have the shape of a gaussian curve too.

It is apparent that the spatial profile of gain is of maximum importance in the design of an amplifier, therefore it is important to design pump lasers that can create the optimum gain distribution inside the active medium. The importance of the gain profile is not exclusive to amplification, it also plays a fundamental role in the performance of a laser oscillator $[34,35]$. 


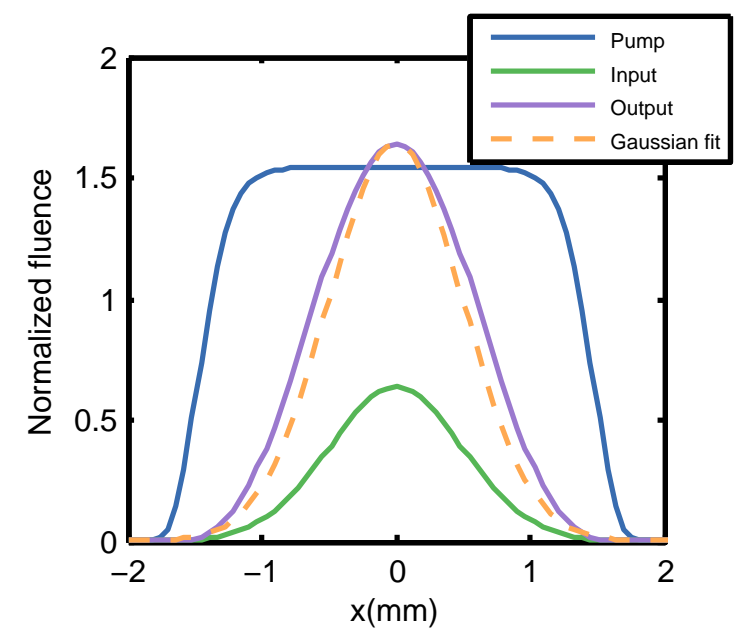

(a)

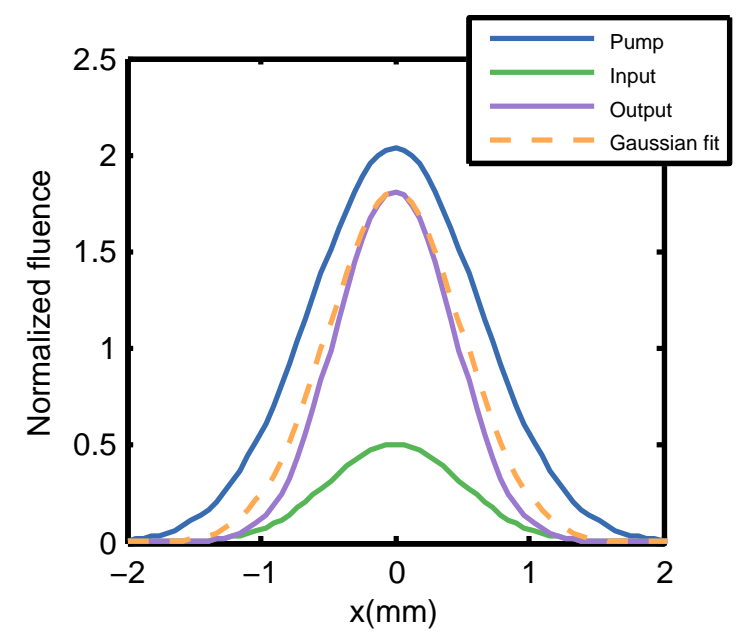

(c)

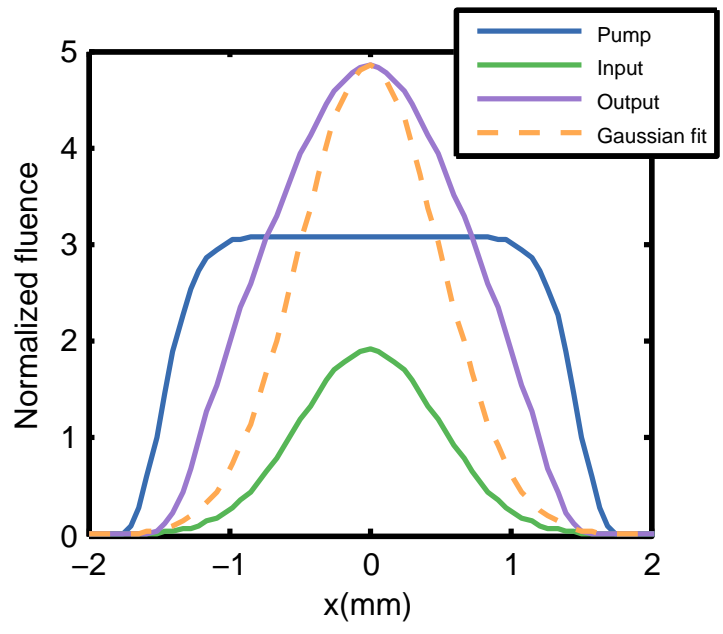

(b)

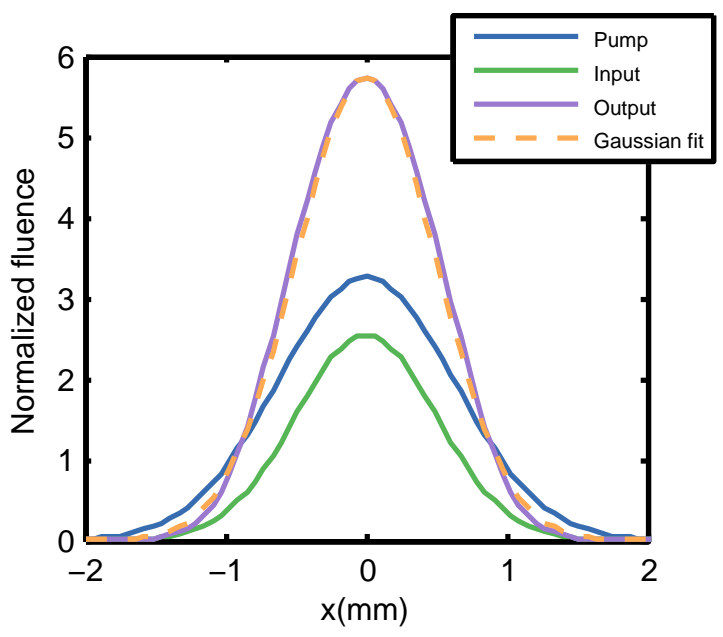

(d)

Figure 2.2: The input gaussian beam (green) is amplified by a gain profile (blue). The output beam (purple) is compared to a gaussian beam (orange dashed line). a) Linear regime for a flat-top gain profile. b) Saturation regime for a flat-top gain profile. (c) Linear regime for a gaussian gain profile. (d) Saturation regime for a flat-top gain profile. 


\subsection{Deformation of the temporal profile}

In order to arrive at the Frantz-Nodvik equation 2.14 it was assumed that the amplification process was instantaneous and time was removed through integration. In order to deal with the time-dependent fluence, we can differentiate eq. 2.19 with respect to time to get the intensity evolution [36]:

$$
I_{\text {out }}(t)=\frac{\exp \left(F_{\text {in }}(t) / F_{\text {sat }}\right)\left(G(t) I_{\text {in }}(t)+F_{\text {sat }} \partial G(t) / \partial t\right)-F_{\text {sat }} \partial G(t) / \partial t}{1+\left(\exp \left(F_{\text {in }}(t) / F_{\text {sat }}-1\right) G(t)\right.}
$$

The gain $G(t)$ becomes time dependent since it is depleted as the pulse travels through the amplifier. It was defined as:

$$
G(t)=\exp \left(\frac{F_{\text {sto }}-\left(F_{\text {out }}(t)-F_{\text {in }}(t)\right)}{F_{\text {sat }}}\right)
$$

While the time-dependent fluences are defined by:

$$
\begin{aligned}
F_{\text {in }}(t) & =\int_{-\infty}^{t} I_{\text {in }}\left(t^{\prime}\right) d t^{\prime} \\
F_{\text {out }}(t) & =\int_{-\infty}^{t} I_{\text {out }}\left(t^{\prime}\right) d t^{\prime}
\end{aligned}
$$

The temporal profile of the pulse plays a role in the performance of amplification, for example, if the fluorescence lifetime is comparable to the duration of the pulse. It is also relevant in OPA or OPCPA amplification chains, where both the temporal profile of the pump and the seed pulse must be able to overlap precisely [37]. In an ultrashort CPA laser the temporal shape of the pulse is fundamental for an efficient compression and it represents a crucial parameter in many applications that require a pulse interacting with matter [38,39].

Just as with the spatial profile, amplification can modify the pulse shape. The front and rear part of the pulse may not experience the same gain, as the photons that arrive first to the amplifier deplete a part of the stored energy and the trailing part of the pulse finds the active medium depleted of energy, creating an unbalance between the front are rear part of the pulse, modifying its shape. In order to 


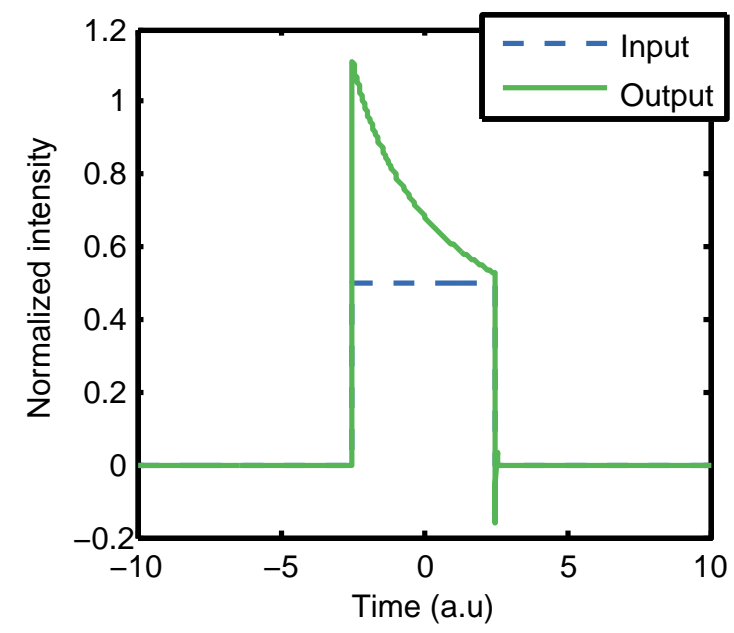

(a)

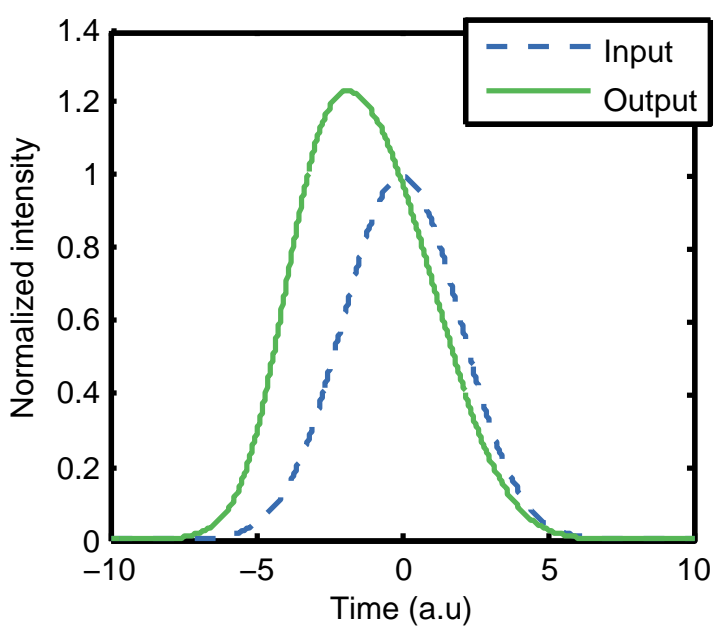

(b)

Figure 2.3: (a) Input pulse (dashed) and amplified pulse (solid) in the case of a rectangularly shaped pulse. (b) Input pulse (dashed) and amplified pulse (solid) in the case of a gaussian shaped pulse.

investigate these effects, equation 2.25 was solved numerically with time as the only dimension for several shapes of the input pulse. The results are pictured in figure 2.3.

An input pulse with a rectangular shape would experience higher gain at the front of the pulse. In figure 2.3(a), the intensity of the front part of the pulse is high enough to deplete notably the energy stored in the active medium, hence the rear part of the pulse experiences low gain. The output pulse becomes trapezoidal-like, and depending on the intensity, the pulse length can diminish.

The case of a gaussian shaped pulse is pictured in figure 2.3(b). For low input intensity, the pulse shape is conserved; the front part of the pulse experiences high gain but the fluence is low and the depletion of the stored energy is not noticeable before the peak of the pulse arrives. However, in the case of saturation and high gain, the front part of the pulse can extract a notable part of the stored fluence and the depletion is not negligible; therefore the front part of the gaussian pulse experiences higher gain than the trailing part, which has the effect of shifting the pulse towards the front of the pulse. 


\subsubsection{Gain narrowing}

The generation of femtosecond pulses require a large gain spectrum. There is a fundamental relation between the temporal duration of a pulse and its spectral bandwidth:

$$
\Delta t \Delta \nu \geq K
$$

$K$ is a parameter dependent on the temporal shape of the pulse. For instance, $K=0.441$ for a gaussian profile and $K=0.315$ for a hyperbolic secant. When the equality on equation 2.29 is satisfied, the pulse is said to be Fourier-limited, which represents the lowest pulse duration possible for a given spectral width. In other cases, the pulse is said to be chirped.

Equation 2.29 also implies that the wider the spectre, the shorter the pulse. However, the spectral width cannot be arbitrarily large and this limit is imposed by the emission cross section of the amplifier, which is a property particular to each material.

In ultra broadband lasers such as Ti:Sa, the pulse duration is one of the key parameters of the laser, thus the conservation of the spectral bandwidth is important. There are two effects that modify the spectrum of the input pulse during amplification: gain narrowing and gain shifting. Gain narrowing induces the loss of spectral bandwidth through amplification while gain shifting leads to a the drift of the central wavelength of the pulse.

Gain narrowing and shifting can also be understood using the Frantz-Nodvik model. The starting point is equation 2.25 , in which time was the only variable considered; the Fourier transform of that equation would describe amplification as a function of the instantaneous frequency. However, in the case of a CPA pulse, the chirping correlates time directly proportional to the instantaneous frequency and in this particular case, we can modify equation 2.25:

$$
I_{\text {out }}(\omega)=\frac{\exp \left(F_{\text {in }}(\omega) / F_{\text {sat }}(\omega)\right)\left(G(\omega) I_{\text {in }}(\omega)+F_{\text {sat }}(\omega) \partial G(\omega) / \partial \omega\right)-F_{\text {sat }}(\omega) \partial G(\omega) / \partial \omega}{1+\left(\exp \left(F_{\text {in }}(\omega) / F_{\text {sat }}(\omega)-1\right) G(\omega)\right.}
$$

The saturation fluence becomes dependent on the frequency, since the emission 
cross section of Ti:Sa is not a constant, and was modelled as a gaussian curve. Equation 2.30 could be solved numerically and was used to support theoretically the evolution of the spectrum of the Ti:Sa laser throughout all the amplification stages in chapter 5 .

\subsubsection{Contrast}

Apart from increasing the energy of the laser beam, the process of amplification introduces noise that distorts the temporal behaviour of the signal. Part of this noise is intrinsic to the amplification process or can be originated by spurious reflections or imperfections in the amplification chain.

The intrinsic part is known as ASE (Amplified Spontaneous Emission). In section 2.1, we assumed that the depopulation of the excited state only depends on the stimulated emission of photons triggered by a seed beam. However, spontaneously emitted photons can also be amplified; the spontaneous emission of a photon can occur at any point in the pumped volume and in any random direction in all solid angles during the entire duration of the pump pulse. The amplification of spontaneous emission follows an equation as 2.2 :

$$
G_{A S E}=\exp \left(\sigma_{A S E} N L_{e f f}\right)
$$

where $\sigma_{A S E}$ is the mean emission cross-section for spontaneous emission and $L_{\text {eff }}$ effective length that an spontaneous emitted photon travels through the active medium. Therefore, ASE becomes relevant if the population inversion is high, that is, in high-gain amplifiers, or if the effective length is large; the distance travelled by the spontaneous emitted photons can exceed the length of the crystal when back-reflections from other optics or the faces of the crystal return them to the crystal. Other sources of reflections, like total reflections inside the active medium can lead to parasitic lasing oscillations, a closed path laser inside the actual crystal, which is detrimental for amplification as it consumes the gain.

In order to quantify the purity of a laser pulse, the concept of temporal contrast is defined as the ratio between the peak intensity of the main pulse and the intensity at a certain time delay. In this way, we can distinguish between femtosecond, picosecond or even nanosecond contrast. 
(d)

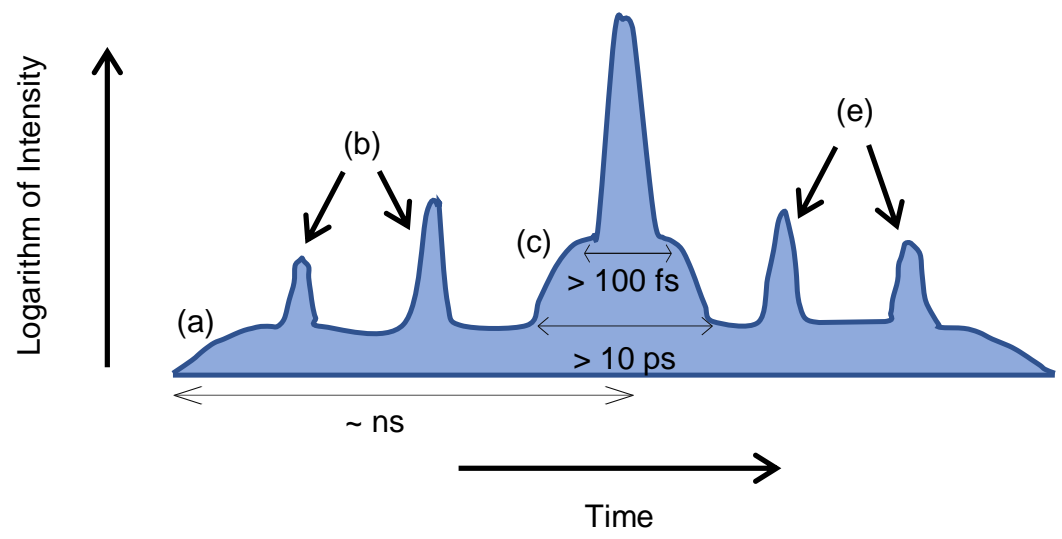

Figure 2.4: Typical temporal structure of an ultrashort laser amplified in a CPA system. (a) ASE pedestal. (b) Pre-pulses. (c) Picosecond pedestal (d) Main pulse. (e) Post-pulses.

In ultra-intense lasers, the temporal quality of the pulses is of maximum importance, especially in applications such as particle acceleration. A few TW laser can be focused to a few microns and achieve a peak intensity in excess of $10^{18} \mathrm{~W} / \mathrm{cm}^{2}$. If the contrast at some point is less than six orders of magnitude, a preceding part of the pulse would present an intensity of at least $10^{12} \mathrm{~W} / \mathrm{cm}^{2}$, which can be enough to interact non-linearly with a material and generate a plasma. If the target is ionized, upon the arrival of the main pulse, the interaction of the laser with the plasma may cause the reflection of the main pulse depending on the plasma density. If the contrast is even lower, the ionization prior to the main pulse may cause a deformation of the surface of the target and the laser will not encounter a clean plane surface, which can be detrimental for processes such as high harmonic generation or proton acceleration [40].

An illustration of temporal artefacts in a typical ultrashort amplified pulse can be observed in figure 2.4. We can observe a pedestal that can span more than 100 ps, a short pedestal close to the main pulse (a few femtoseconds) and pre and post-pulses, pulse replicas that are more intense than the background noise.

These structures have different physical origins. The long pedestal corresponds to the incoherent noise introduced by ASE and, for this reason, it is called incoherent contrast. The ASE is mainly generated in the first, high gain, amplification 
stages. The short pedestal comes from an imperfect recompression after the stretcher/compressor and amplification and is called the coherent contrast. Finally, pre-pulses and post-pulses are replicas of the main pulse due to reflection or diffusion from optical surfaces.

\section{$2.5 \quad$ Non-linear effects}

Amplification of short pulses can lead to the generation of very high intensity pulses, which can induce non-linear effects when the beam traverse some optics or event during its propagation through air.

One of the most harmful non-linear effects is self-focusing. When an intense beam propagates through a medium, it induces a change in the refractive index proportional to the intensity profile; this generates a phase shift that has the same effect as a positive lens. As the beam size decreases, the intensity increases, inducing a more powerful lens, which ends up focusing the laser to a narrow light beam called a filament that damages the medium.

Self-focusing can affect the entire beam or only a small part. These cases are known as whole-beam self-focusing and small-scale self-focusing respectively. Whole beam self-focusing occurs when the focusing effect due to the refractive index exceeds the natural divergence of the beam, causing a catastrophic collapse.

Small-scale self focusing occurs when a part of the beam has higher intensity than its surrounding parts and suffers the effects of localized self-focusing. This creates small amplitude distortions in the beam profile and eventually breaks into several filaments. This effect is caused by hot spots in the beam profile; hot spots are irregularities in the transversal dimension of the beam with higher intensity. In turn, hot spots can be generated by surface aberrations in optical components, dust, etc. In some cases, even noise fluctuations in the beam profile can cause small-scale self-focusing, sometimes inducing beam inhomogeneities that induce local intensities too close to ablation thresholds and reduce significantly the lifetime optical components.

The B-integral is a criteria that measures the risk of a laser to suffer non-linear effects.

$$
B=\frac{2 \pi}{\lambda} \int n_{2} I(z) d z
$$


where $n_{2}$ is the non-linear refractive index that depends on the medium. The value of the B-integral measures the non-linear phase added to the laser beam during amplification over a distance $\mathrm{z}$. The value of this parameter must be less than 2 in order to avoid the build-up of self-focusing, which can lead to optical damage.

Since self-focusing scales with intensity it is more probable to occur in picosecond lasers, but this effect was observed also in a nanosecond Nd:YAG laser during the development of this thesis. 


\section{Chapter 3}

\section{Diode pumping heads}

The success of laser diodes as a mean of pumping lasers was another step towards the emergence of a new generation of high power solid state lasers, providing an increase in efficiency and reliability. Several combinations of semiconductors, such as AsGa, emit naturally in the near infrared, a region of the spectrum which makes them suitable to excite a great number of laser sources, such as $\mathrm{Yb}$ and Nd-doped materials.

In this chapter, we will review the design and performance of a diode pumping head, an arrangement of laser diodes that pump a gain medium, in this case a Nd-doped solid-state rod. The importance of pumping heads lie in that they can be used as building blocks for more complex setups, work in continuous or pulsed mode, and serve as either laser generators or laser amplifiers.

\subsection{Nd-doped materials}

Materials doped with $\mathrm{Nd}^{3+}$ ions have been known since the middle of last century. This rare-earth ion can be implanted successfully in a lot of matrices: YAG, YLF, $\mathrm{YVO}_{4}$, glass, etc. They are well known for their fundamental emission in the near infrared and have an absorption coefficient bandwidth suitable for being pumped by laser diodes, however, $\mathrm{YVO}_{4}$ lasers have short fluorescence lifetime, which reduces its capacity to store energy while the low thermal conductivity of glass lasers prevent them from operating at high average power. In this work, both Nd:YAG and Nd:YLF were used as laser sources, therefore we will make special emphasis 
on them.

\subsubsection{Nd:YAG}

A Nd:YAG crystal was first used as a laser source in the 60s [41] and gained acceptance over the years as it became a source for high power pulses after the invention of Q-switching.

The matrix of the material YAG stands for Yttrium Aluminium Garnet and its chemical composition is $\mathrm{Y}_{3} \mathrm{Al}_{5} \mathrm{O}_{12}$. The crystalline structure of YAG is cubic and the $\mathrm{Nd}^{3+}$ ions substitutes the $\mathrm{Y}^{3+}$ in the lattice; the difference in size between both ions, although small, leads to strain in the YAG if the doping concentration of $\mathrm{Nd}$ is high. The cubic lattice grants isotropic optical and mechanical properties to Nd:YAG.

Nd:YAG is primarily grown using the Czochralski method. This technology is mature and therefore high optical quality Nd:YAG crystals can be grown. This method works by loading a solution of the desired material, rich in the dopant, into a custom growth chamber, where all gases inside are evacuated. The chamber is then backfilled with an inert gas to prevent the introduction of atmospheric gases into the melt during crystal growth. The material is melted, and a thin seed of YAG is introduced into the melted material. The seed crystal is withdrawn at a slow and controlled rate, and crystal and crucible are rotated in opposite directions. The process is repeated, and crystal layers are added with each cycle until the target size is reached. This forms a boule of material that can then be cut and polished into suitable laser media.

Interestingly this material can be produced in a polycrystalline form or ceramic. A powder of the desired material is subjected to extremely high pressure and converted into a transparent polycrystalline ceramic material. The polycrystalline ceramic YAG consists of grains of sizes in the micrometer order and grain boundaries that separate the grains. Creating a ceramic Nd:YAG solves some problems of traditional methods such as the limitation of doping concentration and uniformity, size scalability and most importantly, it enables the joining of Nd:YAG with undoped YAG or other similar materials with interesting properties. We tested both single crystal and ceramic YAG in the laboratory and observed that while 


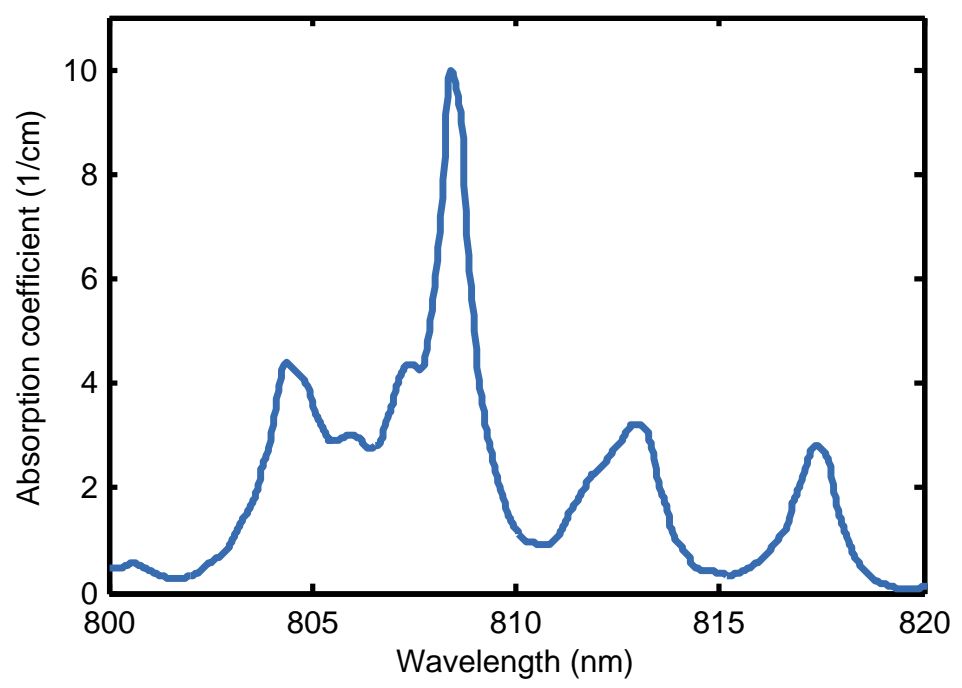

Figure 3.1: Absorption coefficient of Nd:YAG 1\% at. doping

the output power was similar for both materials at the same conditions, ceramic Nd:YAG introduced higher scattering losses as compared to the single-crystal version. This is the reason why all data involving Nd:YAG in this thesis was acquired using crystalline Nd:YAG.

The Nd3+ ion has three electrons in a $4 \mathrm{f}$ subshell which give rise to four levels corresponding to the possible values of angular momentum: ${ }^{4} \mathrm{I}_{9 / 2},{ }^{4} \mathrm{I}_{11 / 2},{ }^{4} \mathrm{I}_{13 / 2}$, ${ }^{4} \mathrm{I}_{15 / 2}$. The lowest energy level is ${ }^{4} \mathrm{I}_{9 / 2}$ which will be considered the ground state and each of these levels is further split by the Stark effect. There is a laser transition at $1064 \mathrm{~nm}$ that occurs between level ${ }^{4} \mathrm{I}_{11 / 2}$ and ${ }^{4} \mathrm{~F}_{3 / 2}$, while the rest of $\mathrm{F}$ and $\mathrm{G}$ bands work as pump bands that decay non-radiatively to this level; especially important is the ${ }^{4} \mathrm{I}_{9 / 2} \rightarrow{ }^{4} \mathrm{~F}_{5 / 2}$ transition which is responsible for the absorption peak at around $808 \mathrm{~nm}$, which has a $220 \mu$ s fluorescence lifetime [42]. Once in the ${ }^{4} \mathrm{I}_{11 / 2}$ level, the electron decays again non-radiatively to the ground level, effectively providing a four level laser system.

Apart from the $1064 \mathrm{~nm}$ transition, the energy level of Nd:YAG can produce other wavelengths. There is a $1338 \mathrm{~nm}$ transition between ${ }^{4} \mathrm{~F}_{3 / 2} \rightarrow{ }^{4} \mathrm{I}_{13 / 2}$ and $946 \mathrm{~nm}$ between ${ }^{4} \mathrm{~F}_{3 / 2} \rightarrow{ }^{4} \mathrm{I}_{9 / 2}$. The cross section of these transitions is about five times weaker than that of the $1064 \mathrm{~nm}$ transition line, and this line must be suppressed [43]. 


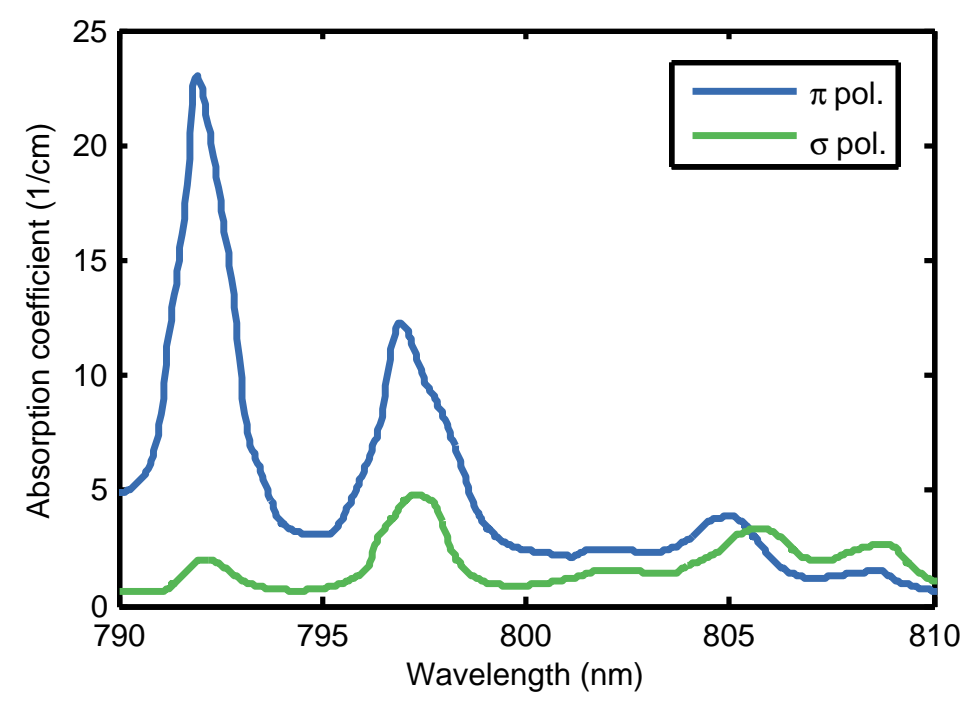

Figure 3.2: Absorption coefficient of Nd:YLF 1.5\% at. doping for ordinary and extraordinary polarizations.

\subsubsection{Nd:YLF}

Nd:YLF is another Nd-doped material that has some interesting properties that differentiate it from Nd:YAG. The matrix is $\mathrm{YLF}\left(\mathrm{YLiF}_{4}\right)$ which is in the hexagonal group. This lattice has a privileged direction (c axis) which induces a natural birefringence in the crystal that, although weak, grants some unique properties to the laser action.

The electronic configuration of Nd:YLF is similar to Nd:YAG, with 3 electrons in a $4 \mathrm{f}$ subshell, and the main laser transition occurs between the same atomic levels ${ }^{4} \mathrm{I}_{9 / 2}$ and ${ }^{4} \mathrm{~F}_{5 / 2}$. However, the lattice modifies the energy of the transition as a function of the polarization: $1053 \mathrm{~nm}$ for the ordinary ray and $1047 \mathrm{~nm}$ for the extraordinary. Another important characteristic is the long lifetime of the upper laser level, $500 \mu \mathrm{s}$, which means that Nd:YLF can be pumped during longer periods of time and potentially store more energy.

The absorption cross section of Nd:YLF is shown in figure 3.2. It presents a high peak near $790 \mathrm{~nm}$. However, the weaker peak around $806 \mathrm{~nm}$ might be more interesting, as it is broader and therefore improves the stability of the laser output, since temperature fluctuations will not affect the absorption of pump power.

YLF is mechanically weaker and presents worse thermal properties than YAG. This means that it is not so useful at very high power operation, where high thermal 


\begin{tabular}{llll}
\hline & \multirow{2}{*}{ Nd:YAG } & \multicolumn{2}{c}{ Nd:YLF } \\
& & $\pi$ pol. & $\sigma$ pol. \\
\hline Wavelength $(\mathrm{nm})$ & 1064 & 1053 & 1047 \\
Lifetime $(\mu \mathrm{s})$ & 220 & 550 & 550 \\
Refractive index & 1.82 & 1.45 & 1.45 \\
Thermal conductivity $(\mathrm{W} / \mathrm{mK})$ & 14 & 6 & 6 \\
dn/dT $\left(10^{-6} K^{-1}\right)$ & 7.3 & -4.3 & -1.2 \\
\hline
\end{tabular}

Table 3.1: Summary of the main optical and thermal properties of Nd:YAG and Nd:YLF relevant for the present study.

loads can cause catastrophic damage to the crystal. However, it has a smaller and negative $\mathrm{dn} / \mathrm{dT}$, which means that the thermal lensing effect in a Nd:YLF laser will be weaker and act as a negative lens.

The most common growth method for Nd:YLF is Czochralski, but due to its anisotropic nature and the difficulties of growing fluoride crystals, it lacks the average optical quality of Nd:YAG and long YLF crystals can cause phase front distortions that decrease the laser performance. The presence of the optical axis allows for the growth of c-cut crystals, where the optical axis is perpendicular to the crystal axis and a-cut crystals, where the optical and crystal axis are parallel.

Table 3.1 summarizes the most important parameters of Nd:YAG and Nd:YLF

\subsection{Diode Pumping heads: design}

Diode-pumped solid-state laser systems are becoming increasingly available thanks to the introduction of new high power diode bars in the near-infrared region of the spectrum. This has allowed the development of high peak and high average power Q-switched lasers with many industrial and scientific applications. Up until recently, the most common method to pump an Nd-doped solid-state laser was to use flash lamps, using high voltage to excite a gas that provides huge amounts of pumping power, which results in pulsed lasers characterized by high energy per pulse (up to several Joules per shot) but at the cost of low repetition rate (10-50 Hz). Moreover, due to their extremely wide emission spectra, the overall 
efficiency of the laser is very low, and the thermal load is increased because of unwanted excitations that only generate heat. In contrast, laser diodes emit a much narrower spectra (a few nanometers), so they can be tuned to match the peaks of the absorption spectrum of the active medium, which greatly improves the overall efficiency of the laser and alleviates thermal effects. Another advantage of the laser diodes is their long lifetime (up to $10^{9}$ shots), which can be sometimes overshadowed by their high cost, although it is decreasing continuously.

The requirements for the operation and housing of laser diodes are significantly different than flash-lamps, hence new methods for pump radiation transport must be developed. The two most common methods for pumping a solid state laser using laser diodes are longitudinal and transversal pumping. In the first method, also known as end-pumping, light from the diodes is directed into the active medium usually in the same direction as the laser light coming from the laser. This scheme is useful when aiming for efficiency and beam quality, as the spacial overlap between the pump light and the $\mathrm{TEM}_{00}$ mode can be maximized. In the other method, transversal or side-pumping, the diodes are arranged surrounding the active medium. This scheme offers some advantages such as high power scalability, stacking a greater number of diodes around the laser rod and a more compact design.

In the end-pumping configuration, the absorbed power distribution resembles the profile of the pump beam and can be easily tuned using optics. The sidepumping configuration lacks this flexibility, and the pump distribution will be the superposition of all the sources surrounding the active medium. Many side-pumping schemes have been proposed over the years, using either cylindrical or rectangular crystals [44]. Still, it is critical to achieve uniform absorbed power distribution over the entire volume of the active medium, not only for good optical performance, but also in order to minimize thermal and mechanical stresses.

\subsubsection{High power pumping head: the PH5400}

In this work, we used a particular design of a diode side-pumped laser chamber, known commercially as PH5400, developed by Proton Laser Applications and built by Monocrom. This laser module is composed of a gain medium, where lasing 


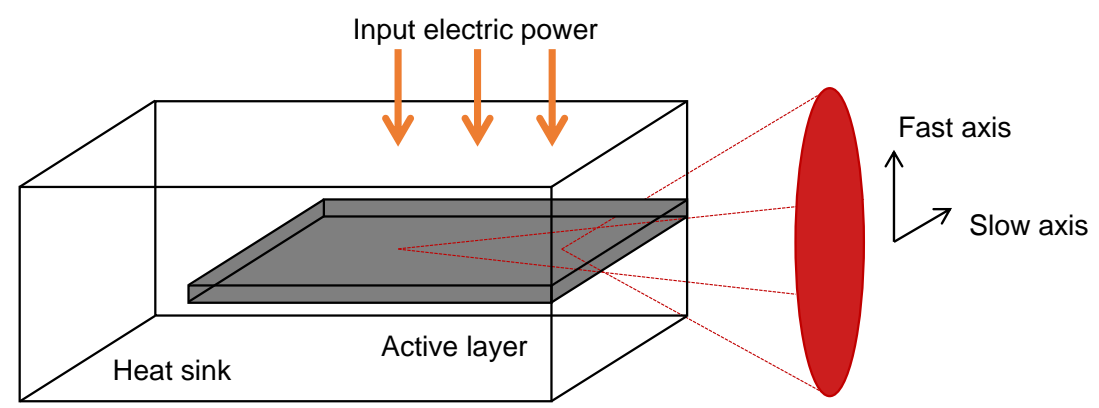

Figure 3.3: Scheme of a typical double heterojunction laser diode featuring the active layer, the heat sink and the electrodes. The emission pattern is an elliptical beam featuring two distinct axes, the fast (vertical) axis and the slow (horizontal) axis.

action takes place surrounded by a plethora of laser diodes whose goal is to pump the laser medium. The energy provided to the laser diodes is usually in high current pulses that are originated inside the PH5400 by a set of especially designed electronics. A water circuit traverses the whole pumping head and cools the laser medium as well as the diodes and the power electronics. Finally, a metal frame fixes all other components to the optical table.

\subsubsection{Laser diodes}

The laser diode is a semiconductor with a $p n$ junction that is powered by an electric current. Optical gain is achieved by stimulated emission inside the active layer. Some faces of this layer are coated as mirrors for the wavelength of the laser diode, which provide a resonator for the stimulated emission to build up.

There are a variety of different laser diodes according to the shape and configuration of the resonator and active layer: double heterostructure lasers, quantum well laser diodes, quantum cascade lasers, Distributed Bragg Reflectors or VCSELs (Vertical-Cavity Surface-Emitting Lasers). Each of these configurations provide particular characteristics to each kind of laser diode.

Due to the size of the emission area of the diodes, they are usually packed in bars, lines of several emitting sources. Diode stacks are multidimensional arrangements of the diode bars. The organization of diode stacks is of great interest for applications that require high power. 


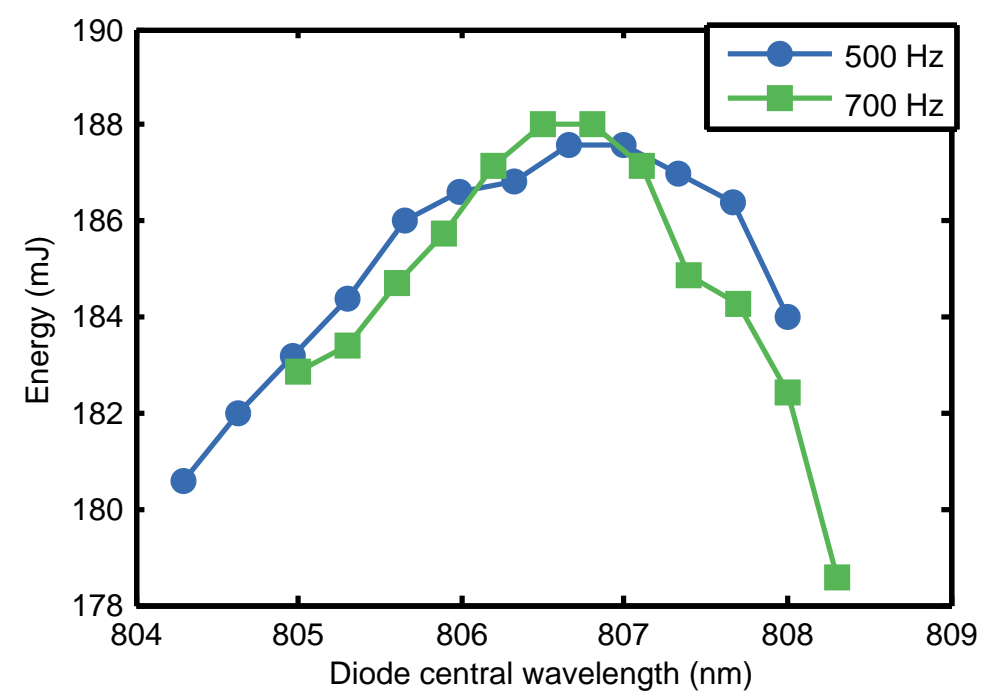

Figure 3.4: Variation of the output pulse energy of a PH5400 as a function of the central wavelength of the pumping diodes. Blue circular dots display data taken at $500 \mathrm{~Hz}$ of repetition rate and the green square dots are data taken at $700 \mathrm{~Hz}$.

The emission wavelength of a laser diode is determined by the bandgap of the semiconductor material chosen for the active layer. The alloying of semiconductor materials to form three element (ternary) or four element (quaternary) compounds allows for the tuning of the wavelength of the laser diode by modifying the relative amount of each element and thus the bandgap. Nowadays, diode lasers cover most part of the visible spectrum and beyond. The emission wavelength depends on the election of the semiconductors used in the active layer. InGaN diodes emit from $380 \mathrm{~nm}$ to $470 \mathrm{~nm}$, AlGaInP emit between $630 \mathrm{~nm}$ and $670 \mathrm{~nm}$, AlGaAs cover the region from $720 \mathrm{~nm}$ to $850 \mathrm{~nm}$, InGaAs from 900 to $1100 \mathrm{~nm}$ and InGaAsP laser diodes emit in the infrared from $1000 \mathrm{~nm}$ up to $1650 \mathrm{~nm}$.

Of all available diodes, in this thesis we are concerned with AlGaAs diodes because their wavelength falls within the absorption cross section of Nd:YAG and Nd:YLF and can be used as pump sources for both Nd-doped materials.

The electrical to optical efficiency of a typical AlGaAs laser diode is $50-60 \%$, therefore, for stacks that can output several thousands of watts of optical power, a cooling system is needed. The diode stacks used in the PH5400 pumping heads are attached to a copper heat sink that includes a principal channel for the flow of water, which branches into a series of microchannels for a more homogeneous 

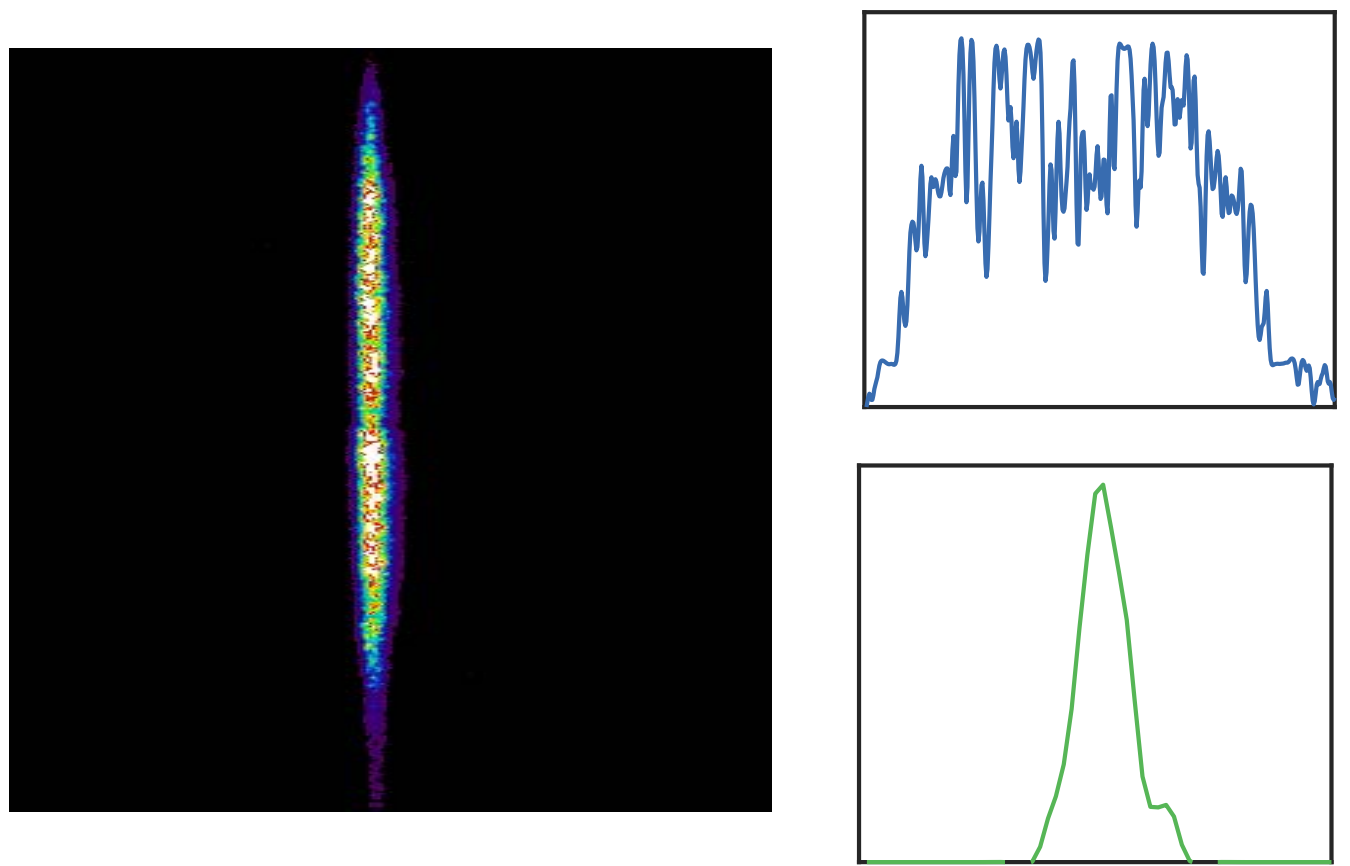

Figure 3.5: Image captured by a CCD camera of the emission pattern of a laser diode bar with its fast-axis collimated. The adjacent figures represent the vertical profile (up, blue) and the horizontal profile (down, green).

cooling of the stack.

An important feature of laser diodes is that temperature also affects their emission wavelength, because the bandgap has a dependence on temperature. Although the emission bandwidth of an individual laser diode is very narrow, differences in manufacturing lead to a spread of the emission spectrum in a laser diode stack. In the case of the PH5400 pumping heads, the diode stacks are water cooled and the temperature of the water enables the fine tuning of the laser diode central wavelength to the peak absorption of Nd:YAG or Nd:YLF, as seen in figure 3.4 , where the energy of the laser experiences around a difference of $5 \%$ for a change of three nanometers in the wavelength of the laser diodes. Since both materials have absorption peaks in the range $805 \mathrm{~nm}-810 \mathrm{~nm}$, the same diodes can be used to pump a pumping head loaded with either active medium

The emission pattern of a laser diode bar is asymmetric due to the rectangular cross section of the active layer which results in an elliptically shaped beam, as 
shown in figure 3.3. The beam has two axes known as fast and slow axis, which points to the difference in divergence between both axes, the fast axis having a larger divergence than the slow one.

The fast axis pattern can be approximated to a Gaussian profile generated by a single point source. On the other hand, the slow axis, although the divergence is lower, is harder to model since there are multiple emitters in the large section and the beam pattern will be the overlap of the beam generated by each emitter. Figure 3.5 shows the emission pattern of one of the diode bars integrated in the stacks used in the PH5400 pumping head, with the fast axis collimated.

A typical model used to describe the emission of laser diodes is to consider the fast axis as a single source with its origin near the surface of the emitter. The slow axis is then modelled using another source but situated at a certain distance from the fast axis source. Although this model of laser diodes as two astigmatic sources has been used, it fails to predict accurately some experimental situations concerning focusing or coupling of a laser diode into a fibre [45].

The polarization of laser diodes is usually parallel to the slow axis. The orientation of the active medium with respect to the diodes is not relevant for a uniform crystal like Nd:YAG but can have an impact in birefringent crystals like Nd:YLF.

\subsubsection{Simulations of pumping distribution}

The performance of the pumping head is closely related to the coupling of the diode laser light to the active medium. An optimally designed pump cavity will allow the rod to absorb the most power from the diodes (barring losses), but if the absorbed light distribution of the rod is not homogeneous or asymmetrical then the actual laser originated from that gain distribution will not have a good quality. The absorbed light distribution depends on many factors: doping of the rod, arrangement of the diodes, etc. Therefore a model of the distribution of absorbed power is very useful in order to predict the optical and thermal behaviour of the pumping head.

The simulations performed are based on a ray-tracing Monte Carlo code where the light radiated by the laser diodes is treated as rays generated with random 
angles and intensity, and weighted according to the emission pattern. These rays are refracted by several surfaces on their way to the active medium where they are absorbed and the sum of the contribution of many rays gives rise to the absorbed light distribution.

Due to the different behaviour of laser diodes in the two dimensions defined by the fast and slow axis, the rays emitted receive a different treatment depending on the direction. Each ray is provided a random direction and its intensity is weighted by the emission pattern of the laser diodes.

The fast axis is modelled as a point source, located at a distance of $7.5 \mathrm{~mm}$ from the centre of the rod. The slow axis is modelled as an extended source of length equal to that of the active layer, with rays originating from a random position along this stripe. The intensity of the ray varies depending on its initial direction as:

$$
I(\theta)=I_{0} \exp \left(-\left(\theta_{f}^{2} / \phi_{f}^{2}+\theta_{s}^{2} / \phi_{s}^{2}\right)\right)
$$

where $\phi_{f}$ and $\phi_{s}$ are related to the fast and slow axis FWHM divergence by $\phi=\phi_{F W H M} \sqrt{2 \ln (2)}$. For the laser diodes inside PH5400 pumping head, the values of divergence are $30^{\circ}$ and $5^{\circ}$ respectively, as stated by the manufacturer.

Apart from the point of origin and direction, each ray is given a random wavelength within the spectrum of the diodes. Since the absorption peak of Nd:YAG and Nd:YLF is narrower than the emission of a diode stack, the absorption will be different for each ray.

For their propagation, each ray $i$ is defined by its initial position $\mathbf{r}_{\mathbf{0}}$, the virtual source of the ray, angle, defined by the unitary vector $\mathbf{k}$, and a real parameter $p$, as in equation 3.2. These rays propagate freely until the intersection with each of the three interfaces. These interfaces are cylindrical surfaces so they can be expressed as equation $3.3, R_{j}$ being their respective radius.

$$
\begin{aligned}
& \mathbf{r}_{\mathbf{i}}=\mathbf{r}_{\mathbf{i} \mathbf{0}}+p \mathbf{k}_{\mathbf{i}} \\
& x^{2}+y^{2}=R_{j}^{2}
\end{aligned}
$$

The intersection between each ray $i$ and surface $j$ is the solution to the system of equations 3.2 and 3.3, which can be solved by numerical methods or reduced 
to a quadratic equation for $p$ (choosing the positive solution, as the ray is going forward) and solved analytically.

On the intersection point between the ray and the surface, the normal vector to the surface $\mathbf{n}$ has to be calculated in order to compute the change of direction suffered by the ray when refracted, according to equation 3.4, Snell's law.

$$
n_{j}|\mathbf{k} \times \mathbf{n}|=n_{j+1}\left|\mathbf{k}^{\prime} \times \mathbf{n}\right|
$$

Solving this vectorial equation will lead to several possibilities for the refracted ray; the solution must maximize the scalar product between the incident and the refracted $\mathbf{k}$ vector and the scalar product between the refracted and the normal vector must be negative [46]. One easier way to obtain a solution, since the vectors $\mathbf{n}$ and $\mathbf{k}$ define the plane of incidence, is to define a vector perpendicular to this plane of and rotate $\mathbf{k}$ around this vector by some angle $\Delta \theta$, defined as the difference between the refracted ray, solution of Snell's law in scalar form and the incident ray, again choosing wisely between the possible solutions.

The same procedure is done for the ray $i$ every time it encounters a surface boundary. Once it has traversed the active medium and reaches the outer surface, where a layer of reflecting material is located, the ray experiences specular reflection and now travels backwards. The process ends when the energy associated to each ray falls below a threshold.

For the calculation of absorption the active medium is divided into elements of volume. Each of these elements is associated to a scalar, representing energy, that grows whenever a ray travels through the volume and is absorbed. In the nonsaturated regime, the energy deposited by the ray within each volume is calculated according to Beer-Lambert law of absorption,

$$
\Delta E=E_{0}(1-\exp (-\alpha \cdot \Delta \mathbf{r}))
$$

where $\Delta E$ is the energy absorbed within the element of volume, $E_{0}$ is the energy of the incoming ray, $\Delta \mathbf{r}$ is the space travelled by the ray inside each volume and $\alpha$ is the absorption coefficient of the active medium, which depends on its dopant concentration and wavelength of the pump source. When the ray travels to the next element of volume, its initial energy $E_{0}$ is diminished by $\Delta E$. 
Once all the rays have been launched, the position of the source of the rays is rotated by $60^{\circ}$ around the longitudinal axis to simulate the adjacent diode stack. The total distribution of absorbed energy within the active medium is calculated by adding the contribution of all individual rays by all diode sources.

The ray tracing code was developed in full 3D formalism. The simulations were made with a mesh of $150 \times 150 \times 12$ nodes representing the active medium, which corresponds to divisions of about $40 \times 40$ microns in the traverse direction for a 6 $\mathrm{mm}$ diameter rod. The longitudinal direction was divided into 12 slices, each of $0.58 \mathrm{~mm}$ of thickness. These partitions provide enough resolution for this kind of simulations. Each laser diode stack was simulated with $10^{5}$ rays.

In order to test the validity of the simulations, an experiment was performed to image the fluorescence of a transversal plane inside the active medium with a CCD camera. The pumping chamber configuration (geometry, distribution and materials) was the closest to those parameters used in the simulations. For this task a 4 -f system was chosen, comprised of two positive lenses of $100 \mathrm{~mm}$ focal length separated $200 \mathrm{~mm}$ and $100 \mathrm{~mm}$ away from the CCD, as well as an aperture in the Fourier plane of the first lens that would work as a filter. The lens closest to the camera was placed over a translation mount in order to achieve the best possible quality of the fluorescence image. Also, to avoid possible noise and image degradation coming from the non-absorbed diode light, a line-filter for $1064 \mathrm{~nm}$ was added to the setup. The field depth of this optical setup is much longer than the thickness of the divisions performed in the simulations, therefore the fine details in the longitudinal direction of the fluorescence emission cannot be observed.

A simulation of a $5 \mathrm{~mm}$ diameter Nd:YAG rod of $0.4 \%$ at. doping can be observed in figure 3.6. In this distribution the absorbed power is fairly uniform in the central area of the beam, throughout all the optical axis. The total absorption of the pumping light was estimated to be $87 \%$. Figure 3.7(b) shows the experimental fluorescence profile and figures 3.7(c) and 3.7(d) the one-dimensional cuts compared to the simulations. Figure 3.7(a) is an integration of figure 3.6 in order to reproduce the depth of field of the CCD camera setup. Good agreement is found between our model and the experimental data, which validates the approximation of the laser diodes as rays originating in the outer surface of the active layer.

A simulation of a $6 \mathrm{~mm}$ diameter Nd:YAG rod of $0.6 \%$ at. doping can be 

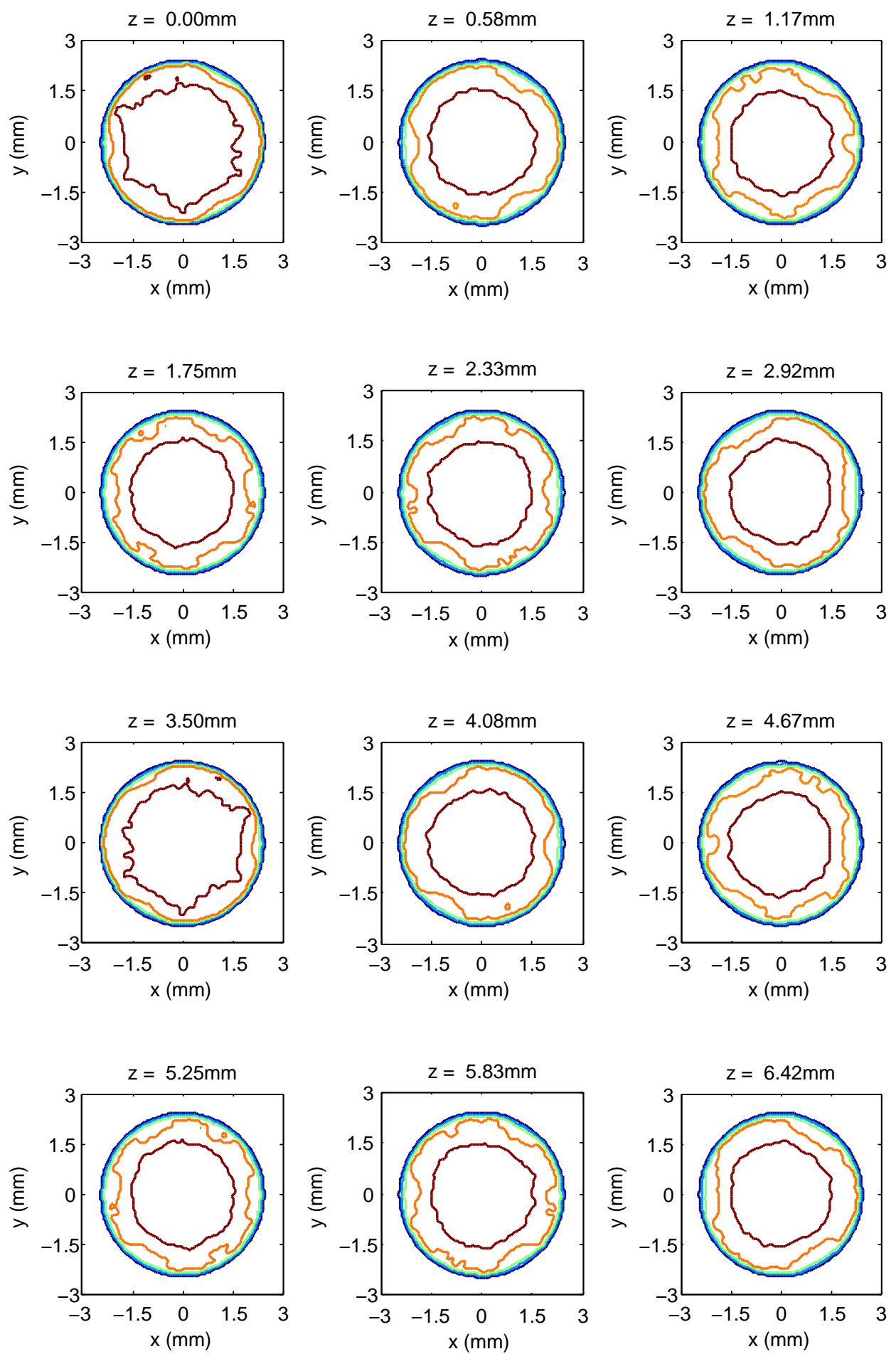

Figure 3.6: Contour plots of a 3D simulation of the absorbed power of a $5 \mathrm{~mm}$ diameter Nd:YAG rod, $0.4 \%$ at. doping. Each z represents a slice along the longitudinal axis of the rod. It covers $7 \mathrm{~mm}$ of the pumped area. 


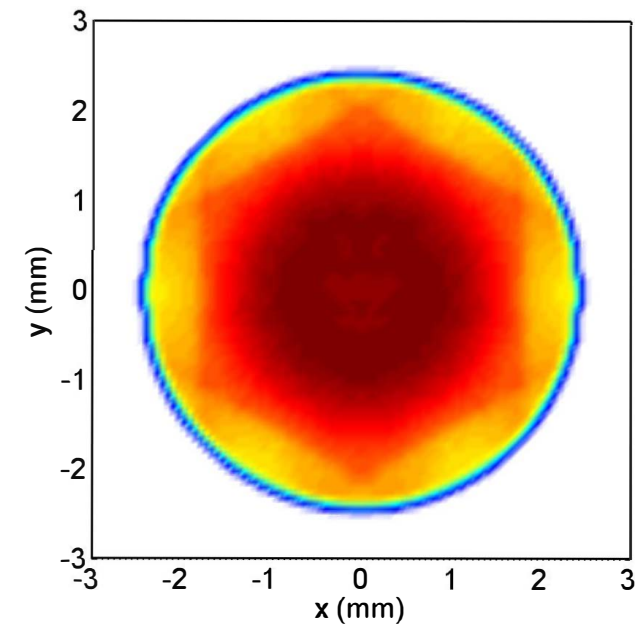

(a)

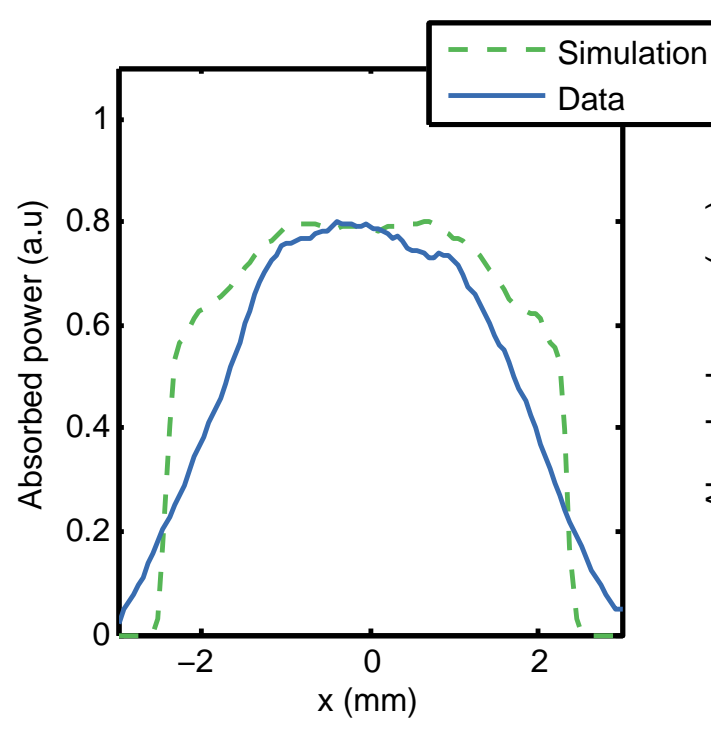

(c)

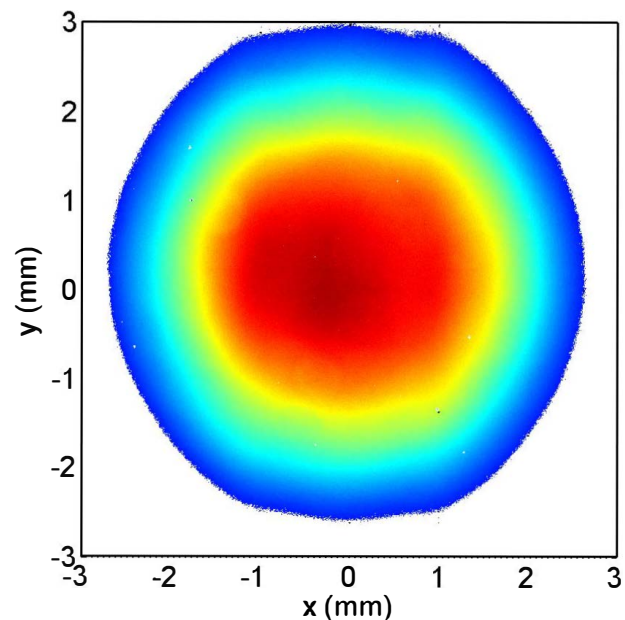

(b)

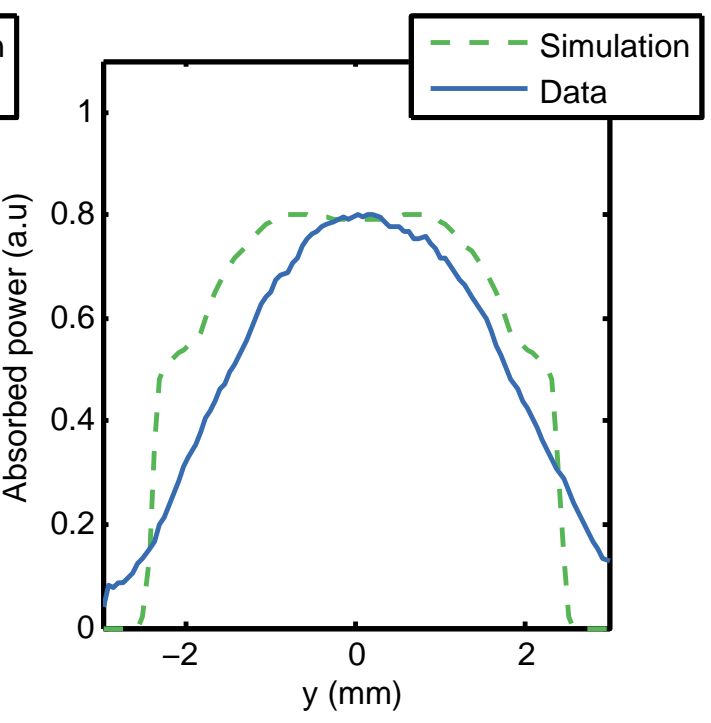

(d)

Figure 3.7: Comparison of simulations and experimental data regarding the absorbed pump profile of the $5 \mathrm{~mm}$ diameter, $0.4 \%$ at. doped Nd:YAG rod: (a) Simulation of the distribution of absorbed power inside the Nd:YAG rod. (b) Measured distribution of fluorescence with a CCD camera. (c) Comparison of horizontal cuts of the simulated (dashed line) and experimental profile (straight line). (d) Comparison of vertical cuts of the simulated (dashed line) and experimental profile (straight line). 

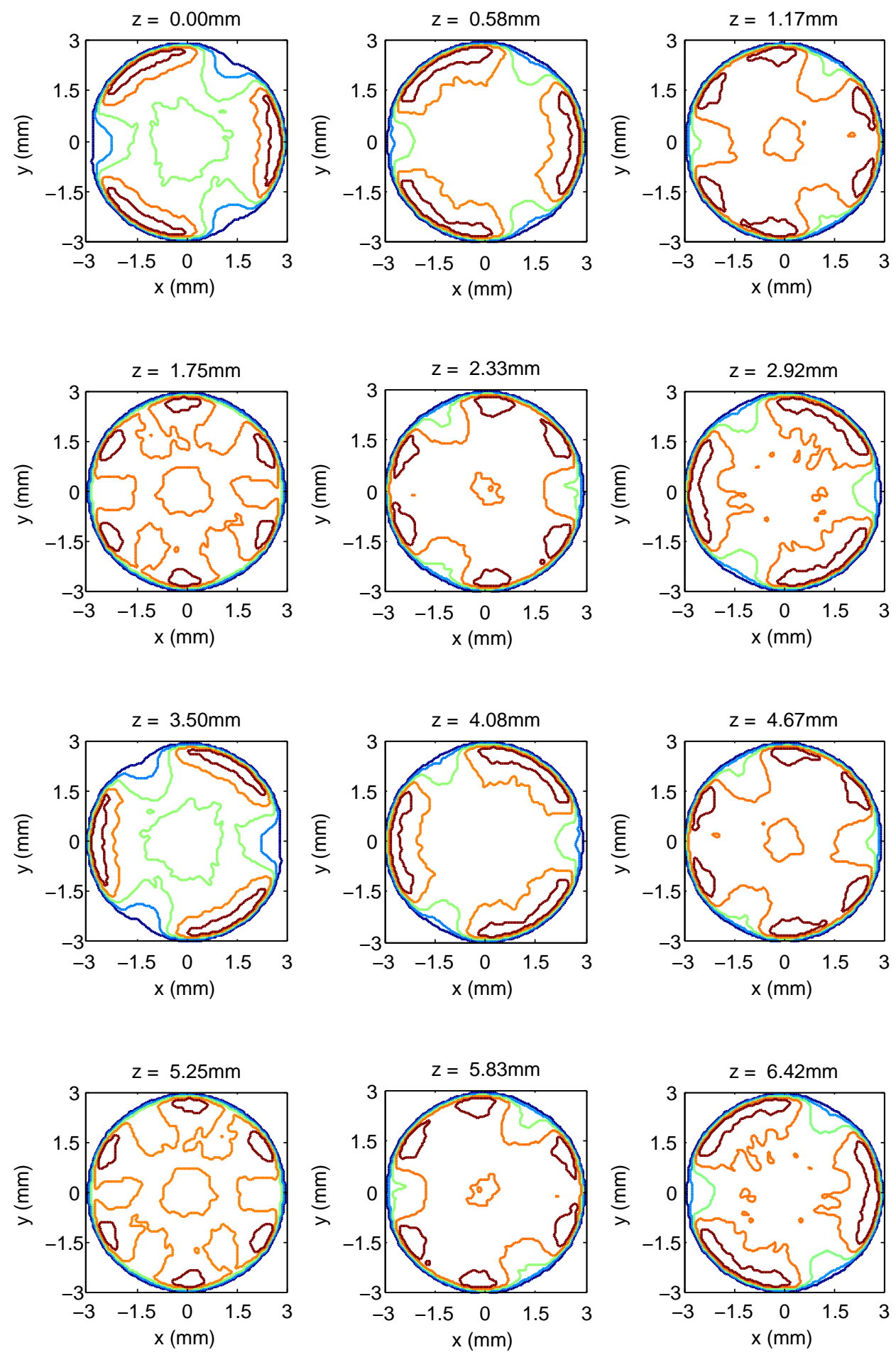

Figure 3.8: Contour plots of a 3D simulation of the absorbed power of a $6 \mathrm{~mm}$ diameter Nd:YAG rod, $0.6 \%$ at. doping. Each $\mathrm{z}$ represents a slice along the longitudinal axis of the rod. It covers $7 \mathrm{~mm}$ of the pumped area. 


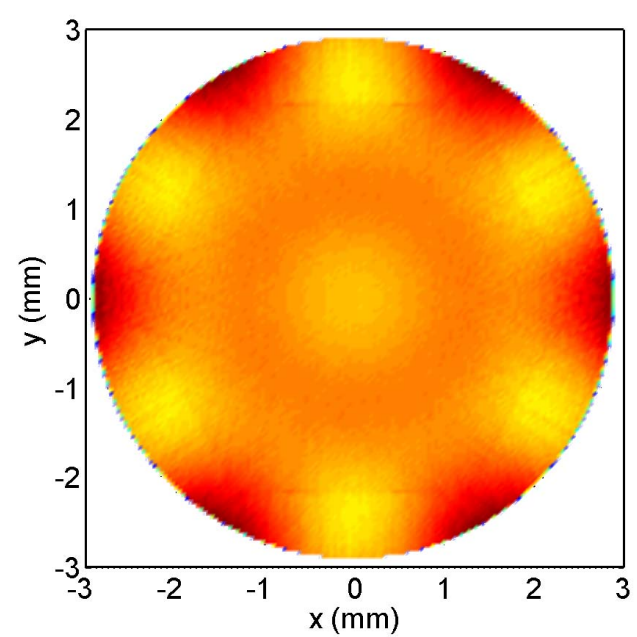

(a)

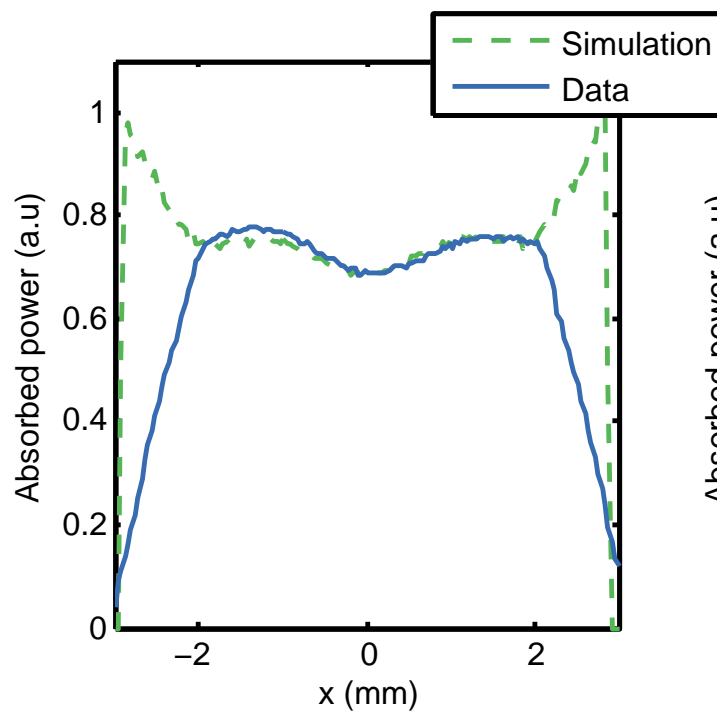

(c)

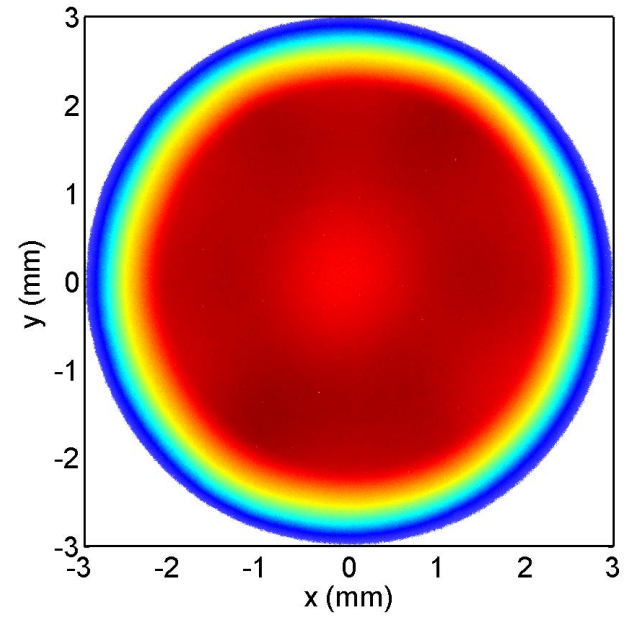

(b)

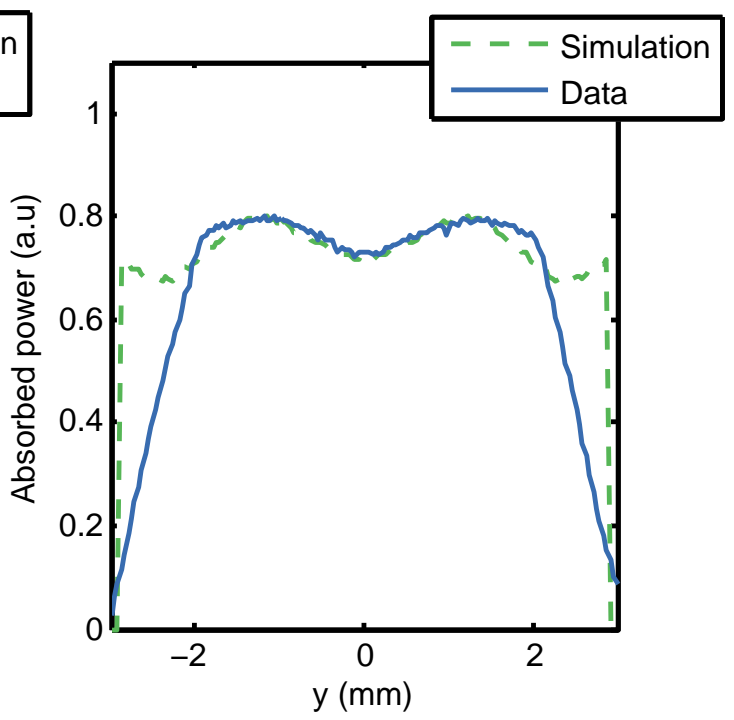

(d)

Figure 3.9: Comparison of simulations and experimental data regarding the absorbed pump profile of the $6 \mathrm{~mm}$ diameter, $0.6 \%$ at. doped Nd:YAG rod: (a) Simulation of the distribution of absorbed power inside the Nd:YAG rod. (b) Measured distribution of fluorescence with a CCD camera. (c) Comparison of horizontal cuts of the simulated (dashed line) and experimental profile (straight line). (d) Comparison of vertical cuts of the simulated (dashed line) and experimental profile (straight line). 

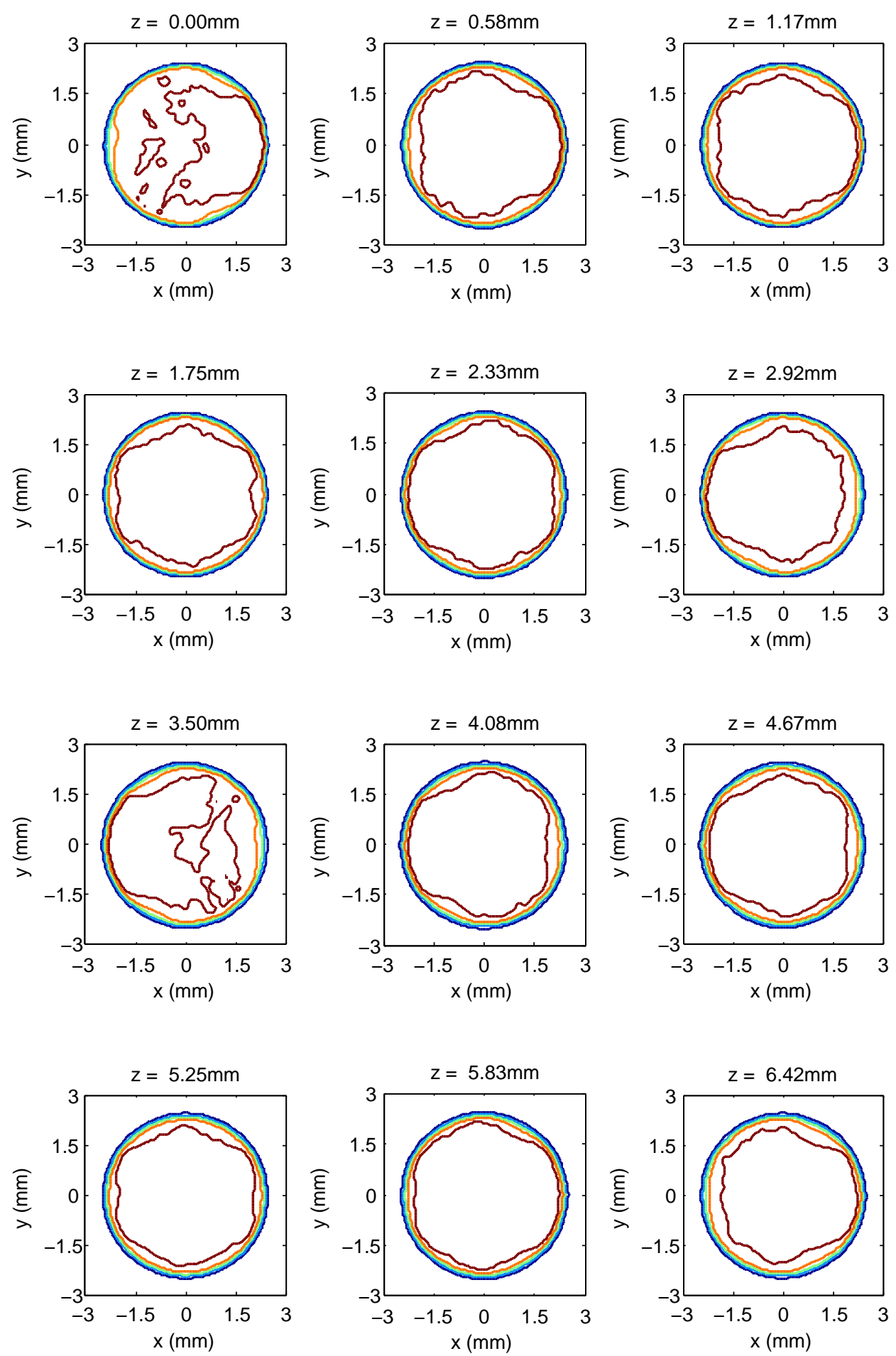

Figure 3.10: Contour plots of a 3D simulation of the absorbed power of a $5 \mathrm{~mm}$ diameter Nd:YLF rod, $0.8 \%$ at. doping. Each z represents a slice along the longitudinal axis of the rod. It covers $7 \mathrm{~mm}$ of the pumped area. 


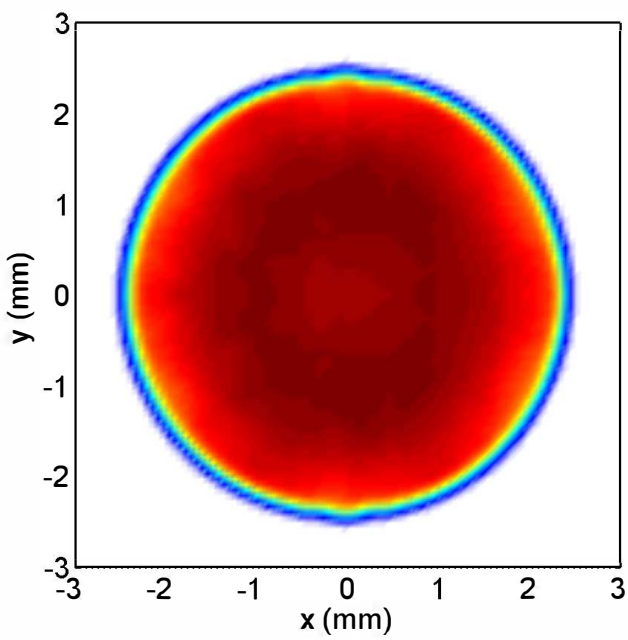

(a)

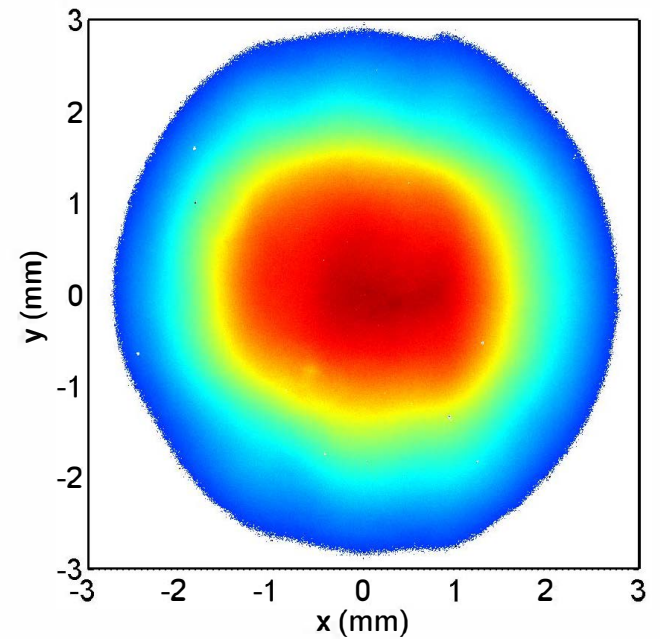

(b)

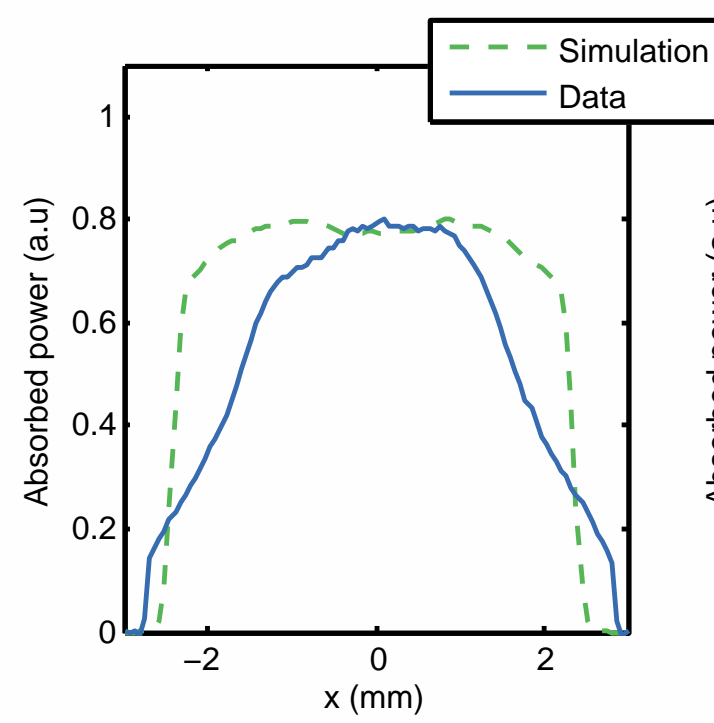

(c)

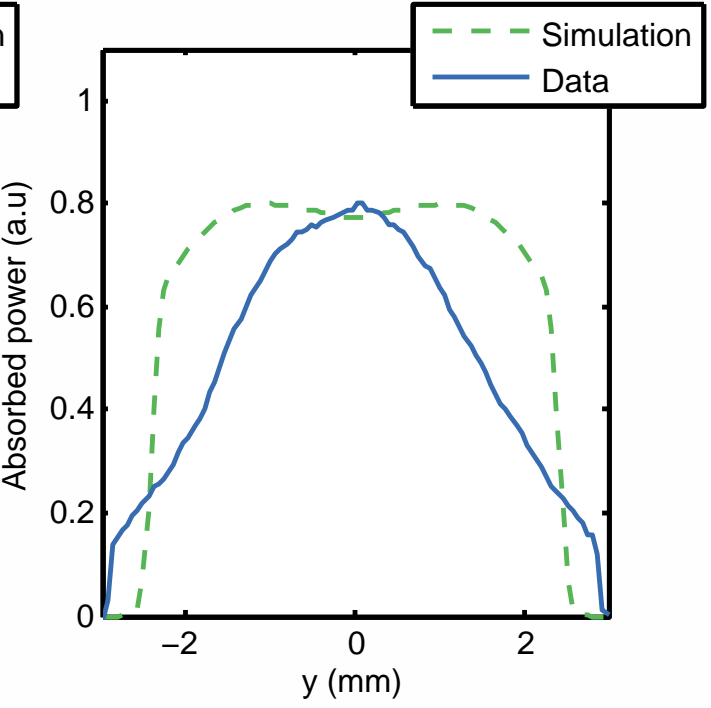

(d)

Figure 3.11: Comparison of simulations and experimental data regarding the absorbed pump profile of the $5 \mathrm{~mm}$ diameter, $0.8 \%$ at. doped Nd:YLF rod: (a) Simulation of the distribution of absorbed power inside the Nd:YLF rod. (b) Measured distribution of fluorescence with a CCD camera. (c) Comparison of horizontal cuts of the simulated (dashed line) and experimental profile (straight line). (d) Comparison of vertical cuts of the simulated (dashed line) and experimental profile (straight line). 
observed in figure 3.8. For this particular diode distribution, higher gain zones are predicted to be located in the outer zones of the crystal where the light emitted by the pumping diodes overlaps. The total absorption of the pumping light was estimated to be $89 \%$. Figure 3.9(b) shows the experimental fluorescence profile and figures 3.9(c) and 3.9(d) the one-dimensional cuts compared to the simulations. The outer regions of the Nd:YAG rod could not be imaged properly because of diffraction and finite apertures, because of the length of the active medium. However, the same features can be observed, mainly the higher gain in the outer zones of the crystal, due to the superposition of the light coming from two adjacent diodes, and lower gain on the central zone. This can be the consequence of the absorption coefficient being high enough so that the light from all the diodes, although it overlaps in the centre of the rod, has been absorbed in the outer regions. This issue can be fixed by lowering the doping concentration of the active medium or decreasing the Nd:YAG rod diameter, at the expense of absorption efficiency.

The simulation of a $5 \mathrm{~mm}$ diameter Nd:YLF rod of $0.8 \%$ at. doping can be observed in figure 3.10. Since the absorption of the pump light by the crystal depends on the polarization of the electric field of the diodes (see figure 3.2) the orientation of the diodes in the PH5400 pumping head will influence the absorbed pump distribution, and create some local asymmetries, particularly at the planes where the diodes are present. It is noticeable how the pump distribution is more homogeneous throughout the transversal plane of the rod as compared to Nd:YAG, due to the fact that the refraction index is smaller and the refraction is softer at the outer surface of the rod. Figure 3.11(b) shows the experimental fluorescence profile and figures 3.11(c) and 3.11(d) the one-dimensional cuts compared to the simulations. The image captured by the camera fails to reproduce the simulations, this could be due a coupling of unabsorbed diode light because it was taken without the narrow-pass filter. However, some features can be noticed such as the asymmetry induced by the polarization of the pump diodes. The total absorption of the pumping light was estimated to be $92 \%$. 


\subsection{Thermal induced effects}

In high power laser operation, unwanted effects begin to appear as the active medium heats up. These effects influence critically the optical behaviour of the laser and must be taken into account in the design stage. Heating is caused by phonons. In the particular case of Nd:YAG/YLF this percentage of pump light that converts into heat is close to $25 \%$, which for a PH5400 working at $100 \mathrm{~Hz}$ is about $90 \mathrm{~W}$ of heat that must be dissipated. Other effects such as quenching can add to the total heat generated. Furthermore, there is a part of the absorbed light that has a probability of decaying non-radiatively, which results in a measurable difference in the thermal load of the active medium in the presence and absence of laser radiation.

The temperature gradients produced in the gain material as a result of heating can lead to stress fracture, which represents the ultimate limit in average power obtainable from a laser material. Below the stress fracture limit, thermal lensing and birefringence adversely affect the output beam quality

\subsubsection{Thermal lensing}

Thermal lensing is an optical distortion caused by an inhomogeneous temperature distribution inside the active medium. In a side-pumped rod, cooling is only possible at the outer surface, so the temperature at the centre of the rod will be higher and will decrease towards the outer surface. This effect will induce a gradient of the refractive index and the laser rod will act as a lens. Knowledge and management of thermal lensing is of vital importance for the performance of the pumping heads, whether working inside a resonator or as amplifiers.

The change in the refraction index has three major contributions: the temperature gradient, mechanical stress and deformation of the end faces of the rod. Temperature induces a change in the refractive index through its temperature dependence, while stress modifies the refractive index through the elasto-optic coefficients and the end faces of the rod acquire a small curvature thanks to thermalinduced expansions in those areas. In the case of Nd:YAG, the contribution of the temperature-induced change is the most dominant while the mechanical contributions adds up less than $20 \%$ of the total variation of the refractive index [47]. 
Even if it is minor, the deformation of the end faces of the crystal is relevant when those faces are coated, as it could result in weakening the layers and diminish the effective damage threshold of the rod faces. In the case of the PH5400 pumping heads, the pumped volume is placed far from the end faces of the rod in order to minimize this effect.

We will consider that the active medium is a cylindrical rod and that the absorbed pump power distribution is homogeneous in order to arrive at a closed expression for the focal length of the thermal lens. The thermal behaviour of the rod is described by the heat equation in cylindrical coordinates:

$$
\frac{d^{2} T}{d r^{2}}+\frac{1}{r} \frac{d T}{d r}+\frac{d^{2} T}{d z^{2}}=-\frac{Q}{K}
$$

where $K$ is the thermal conductivity of the material and $Q$ is the heat generated inside the crystal per unit of volume. In order to simplify the solution, we will consider that the pump is uniform throughout the axis of the rod and drop the $\mathrm{z}$ dependent term. Solving the differential equation 3.6, the solution for the radial temperature distribution is:

$$
T(r)-T(R)=\frac{Q}{4 K}\left(R^{2}-r^{2}\right)
$$

where $R$ is the radius of the rod. The heat generated is provided by the portion of the absorbed pump power that is converted to heat, so we can express the heat per unit of volume as:

$$
Q=\frac{\eta P_{\text {pump }}}{\pi R^{2} L}
$$

where $\eta$ is the percentage of absorbed pump power that is converted to heat and $L$ is the length of the rod. For most laser systems, this number can be approximated by the quantum defect $\eta=h \nu_{\text {pump }} / h \nu_{\text {laser }}$, which is the ratio between the energy of a pump photon and a laser photon. In order to calculate the change of the refractive index with respect to temperature, we can expand the refractive index $n$ in a Taylor series and take terms up to the first order:

$$
n(r)=n_{0}+(T(r)-T(R))\left(\frac{d n}{d T}\right)
$$

Substituting equations 3.7 and 3.8 in 3.9 


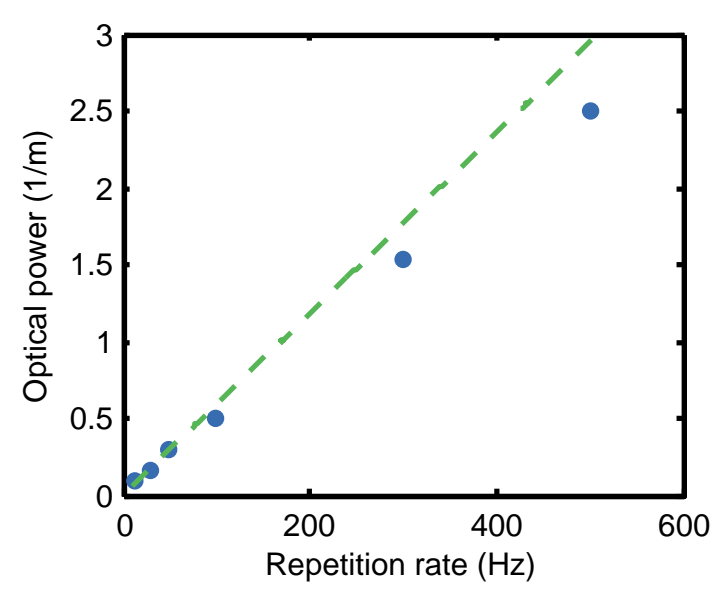

(a)

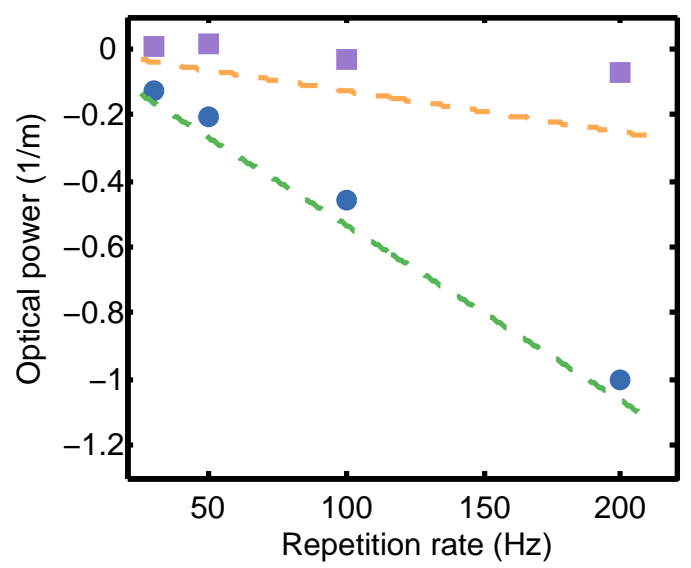

(b)

Figure 3.12: (a) Experimental data (blue dots) of the optical power of the thermal lens created in a PH5400 hosting a Nd:YAG rod. (b) Experimental data of the optical power of the thermal lens created in a PH5400 hosting a Nd:YLF rod. Blue circular dots are values measured for the $\pi$ axis the and purple square dots are values measured for the $\sigma$ axis. Green and orange dashed lines are theoretical values calculated with Eq. 3.12.

$$
n(r)=n_{0}-\frac{\eta P_{\text {pump }}}{4 \pi R^{2} K L}\left(\frac{d n}{d T}\right) r^{2}
$$

This expression is similar to a gradient index lens with a radial dependence on the refractive index

$$
n(r)=n_{0}\left(1-\frac{\alpha r^{2}}{2}\right)
$$

In the paraxial approximation, which occurs when $R \ll L$, it can be shown that the focal length $f$ of such an index distribution is $f^{-1}=\alpha n_{0} L$. Comparing with equation 3.10 we can express the thermal lens as:

$$
f_{\text {thermal }}=\frac{2 K \pi R^{2}}{\eta P_{\text {pump }}}\left(\frac{d n}{d T}\right)^{-1}
$$

A similar expression can be reached through a more exhaustive derivation by Lausten and Balling [48].

In order to measure thermal lensing, we built a setup in which a collimated He-Ne laser traverses the rod inside a PH5400 pumping head. A camera scans the 
size of the beam in several positions close to the beam waist of the He-Ne laser; then a parabolic fitting of the beam size data retrieves the focal length of the rod. We used a Nd:YAG rod pumped by $200 \mu \mathrm{s}, 100 \mathrm{~A}$ and we varied the repetition rate up to $500 \mathrm{~Hz}$. Data is presented in figure 3.12(a).

In the case of Nd:YLF, each optical axis has different thermo-optical properties (table 3.1), therefore the thermal lens behaviour is different if the rod is c-cut. The beam will experience astigmatism. Data is presented in figure 3.12(b).

Equation 3.12 was found to be in good agreement with experimental conditions for both Nd:YAG and Nd:YLF.

\subsubsection{Birefringence}

Birefringence is another optical distortion caused by the same principle that produces thermal lensing. The temperature gradient caused by the absorption of pump light, described by equation 3.7 for the case of a cylindrical rod, induces mechanical stress in the rod, since the centre is hotter and tends to expand, leading to the accumulation of tension at the edge, and affecting the refractive local index. This effect induces a radial variation of the refractive index, so light travelling through the rod polarized along the radius experiences a different phase than light polarized tangentially, similarly to the effect of a waveplate. Therefore, a stressed rod induces depolarization on a beam of linearly polarized light that traverses it.

In an amplifier, this effect induces a severe degradation of the gain. In an oscillator, especially one with an intracavity polarizer for Q-switching, light experiences double-pass depolarization which is rejected by the polarizer, building up losses in the cavity and decreasing optimal performance. This problem can be solved by introducing a quarter waveplate between the active medium and the cavity mirror so that the polarization is rotated $\pi / 2$ before passing again through the active medium [49].

In order to measure depolarization induced by birefringence a setup was mounted in which a He-Ne laser traversed the pumped rod inside a PH5400 and a pair of polarizers, one situated before and the other after the active medium. Depolarization was calculated as the ratio of transmitted to incident power and was measured for $5 \mathrm{~mm}$ diameter Nd:YAG and Nd:YLF rods at different repetition rates (see figure 


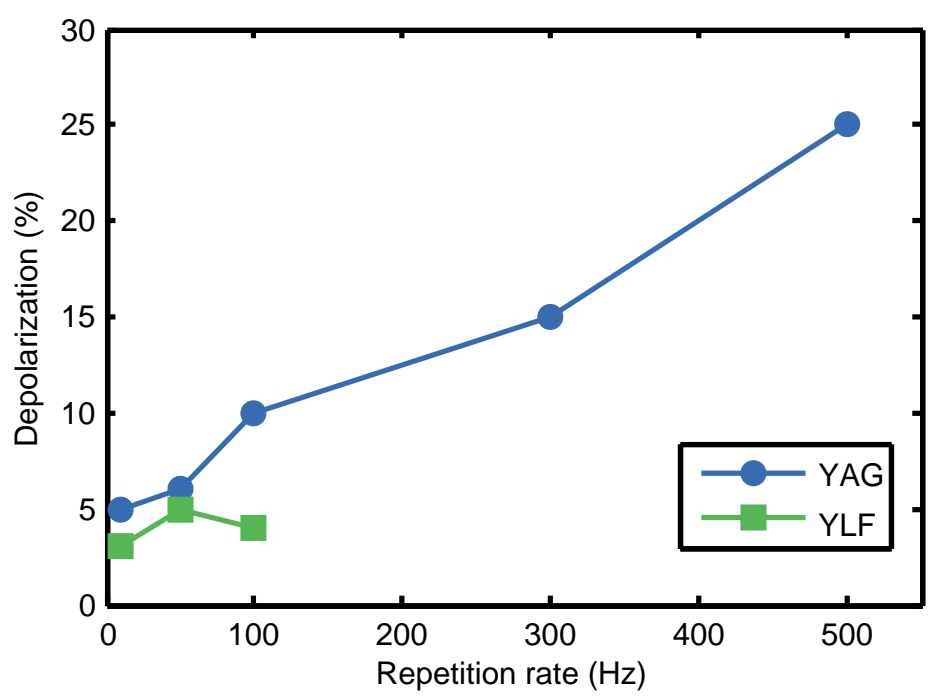

Figure 3.13: Data of depolarization in a pumping head working as a function of the repetition rate. Blue: PH5400 with a 5mm diameter Nd:YAG rod. Green: PH5400 with a $5 \mathrm{~mm}$ diameter Nd:YLF rod.

3.13). Nd:YAG experiences up to $25 \%$ depolarization at $500 \mathrm{~Hz}$, while the effect in Nd:YLF is nearly negligible due to its natural birefringence.

\subsection{Diode Pumping heads: QCW performance}

There are many parameters that rule the behaviour of a pumping module, and their knowledge is key to predict its optical performance. In order to work as a laser, the pumping heads require a resonator. A laser resonator must consist of at least two mirrors and a gain medium. The most basic mode of operation is continuous wave $(\mathrm{CW})$, where the pump laser works continuously and so does the laser. A similar mode of operation is quasi continuous wave (QCW) where the pump and the laser source is pulsed, which produces a train of pulses with a typical temporal length of microseconds, depending on the duty time of the pumping diodes.

\subsubsection{Theoretical description of QCW performance}

We have developed a theoretical model for the QCW performance in order to determine experimentally the relevant parameters of the laser. In this thesis, Nd:YAG and Nd:YLF are used in QCW mode. Both materials are good representatives of a 
four-level laser system. The behaviour of the laser in continuous or pulsed mode can be described by an extension of the space-independent rate equations.

$$
\begin{array}{r}
\frac{d N}{d t}=\Gamma_{p}-\frac{\sigma c}{V} \phi N-\frac{N}{\tau_{f}} \\
\frac{d \phi}{d t}=\sigma c \phi N-\frac{\phi}{\tau_{c}}
\end{array}
$$

where $\mathrm{V}$ is the total volume of the rod. The rate of change of the population inversion $N$ as a function of time is directly proportional to the pumping rate $\Gamma_{p}$, and inversely proportional to the stimulated emission and the inverse of the spontaneous decay, described by the fluorescence lifetime $\tau_{f}$. On the other hand, the rate of flux change is directly proportional to the stimulated emission but diminishes due to some losses with units of time that will be associated to a parameter called photon lifetime of the resonator, $\tau_{c}$.

The resonator is fundamental for the lasing action. The mirrors of the cavity provide feedback for the stimulated emission to grow as it passes through the active medium and is amplified. We can make a model of a resonator by considering two mirrors of reflectivity $R_{1}$ and $R_{2}$ and a gain medium of length $L$. The change of flux when traveling through the active medium is (equation 2.1):

$$
d \ln \phi=\sigma N d z
$$

In a single roundtrip of the resonator the flux of photons is amplified in the gain medium, bounces in a mirror, traverses again the gain medium and bounces on the other mirror. The ratio of the input and final fluxes in the resonator is therefore:

$$
\frac{\phi_{\text {out }}}{\phi_{0}}=\exp (\sigma N) R_{1} \exp (\sigma N) R_{2}=R_{1} R_{2} \exp (2 \sigma N L)
$$

If there is population inversion, the ratio is positive and the photon flux is amplified in each pass through the gain medium. The term $\sigma N$ is the small signal gain $g$ (see chapter 2). Up until now we had assumed that there are no losses present in the resonator apart from the transmission of the mirrors. Typically there are additional losses, such as absorption inside the active medium or other mechanisms such as Fresnel reflections or scattering. We can introduce a term $\delta$ in the gain to account for the absorption losses: 


$$
\frac{\phi_{\text {out }}}{\phi_{0}}=R_{1} R_{2} \exp (2 g l-\delta)
$$

An interesting situation arises when the rate is zero, in which case, the flux of photons remains constant and there is no laser action. This is called the threshold condition:

$$
2 g l-\delta=-\ln \left(R_{1} R_{2}\right)
$$

Typically, in a resonator one of the mirrors is $100 \%$ reflective while the other mirror, known as the output coupler, has some reflectivity less than one, $R$, so that a portion of the photons can leave the resonator. Furthermore, we can introduce reflection or scattering losses in this term as $\delta_{R}$. If the losses are small $\ln \left(1-\delta_{R}\right)=\delta_{R}$ and we can group it with the absorption losses in the term $\delta$. Then we can express the threshold condition as:

$$
2 g l=\delta-\ln R
$$

At threshold, the flux and the population inversion do not change. We can take advantage of this fact in order to calculate the value of the population inversion. The population inversion at threshold, also known as the critical population, can be calculated from equation 3.14 by setting $\frac{d \phi}{d t}=0$

$$
N_{t h}=\frac{1}{c \sigma \tau_{c}}
$$

It depends on the cross section of the laser and the photon lifetime in the cavity, $\tau_{c}$. The meaning of $\tau_{c}$ can be exposed by substituting equation 3.19 on 3.20 :

$$
\tau_{c}=\frac{2 L}{c} \frac{1}{\delta-\ln R}
$$

This quantity accounts for the losses of the cavity in one roundtrip. The critical pump rate to achieve the threshold can be obtained substituting equation 3.20 in 3.13 , considering $\frac{d N}{d t}=0$ and $\phi=0$ :

$$
\Gamma_{t h}=\frac{N_{c r i t}}{\tau_{f}}
$$


This is the minimum pump rate that must be applied to overcome the loss of population of the upper laser level by spontaneous emission.

The output power of the resonator can be calculated as the rate of flux loss from the output mirror $P_{\text {out }}=h \nu \frac{d \phi}{d t}$. Substituting 3.21 into equation 3.14:

$$
\frac{d \phi}{d t}=\sigma c N \phi-\frac{c \delta}{2 L} \phi+\frac{c}{2 L} \ln R \phi
$$

We can identify the term $\frac{c}{2 L} \ln R \phi$ as the rate of flux lost from the mirror. Therefore, the output power is:

$$
P_{\text {out }}=\frac{c}{2 L}(h \nu) \ln R \phi
$$

Now we can calculate the steady-state values for the flux and population inversion from equation 3.13 and 3.14 by setting $\frac{d \phi}{d t}=\frac{d N}{d t}=0$.

$$
\begin{array}{r}
N_{0}=N_{c r i t}=\left(\frac{\delta-\ln R}{2 \sigma L}\right) \\
\phi_{0}=V_{a} \tau_{c}\left(\Gamma_{p}-\frac{N_{0}}{\tau_{f}}\right)
\end{array}
$$

Interestingly, the population at threshold is also the population inversion in the steady state. Operating and introducing equation 3.22 in 3.26

$$
\phi_{0}=\frac{S(\delta-\ln R)}{\sigma} \frac{\tau_{c}}{\tau_{f}}\left(\frac{\Gamma_{p}}{\Gamma_{t h}}-1\right)
$$

Which can be expressed as a function of more empiric variables, the pump power and threshold power.

$$
\eta_{p} P_{\text {pump }, t h}=A_{\text {pump }} L\left(h \nu_{\text {pump }}\right) \Gamma_{p, t h}
$$

where $\eta_{p}$ is the efficiency of conversion of pump power into absorbed power to the active medium, $V_{\text {pump }}$ is the pumped volume and $h \nu_{\text {pump }}$ is the energy of a pump photon. Introducing this into equation 3.27:

$$
\phi_{0}=\frac{A(\delta-\ln R)}{\sigma} \frac{\tau_{c}}{\tau_{f}}\left(\frac{P_{p u m p}}{P_{t h}}-1\right)
$$


Now the output power can be expressed in terms of the pump power introducing equation 3.29 into 3.24

$$
P_{\text {out }}=A \frac{h \nu}{\sigma \tau_{f}} \ln R\left(\frac{P_{\text {pump }}}{P_{\text {th }}}-1\right)
$$

We can define the saturation intensity as;

$$
I_{s}=\frac{h \nu}{\sigma \tau_{f}}
$$

Therefore, we can express the output power as:

$$
P_{\text {out }}=-A I_{\text {sat }} \ln R\left(\frac{P_{\text {pump }}}{P_{\text {th }}}-1\right)
$$

This equation relates the output power of a $\mathrm{QCW}$ laser to the pump power and the reflectivity of the output power. The rest of the parameters are intrinsic features of the active medium. Laser slope efficiency is defined as:

$$
\eta_{s}=\frac{d P_{\text {out }}}{d P_{\text {pump }}}
$$

Inserting this definition into equation 3.30, we obtain:

$$
\eta_{s}=A \frac{h \nu}{\sigma \tau_{f}} \ln R \frac{1}{P_{t h}}
$$

Inserting equation 3.28 into 3.34 and operating,

$$
\eta_{s}=\eta_{p}\left(\frac{A}{A_{\text {pump }}}\right)\left(\frac{h \nu}{h \nu_{\text {pump }}}\right)\left(\frac{2 \ln R}{\delta-\ln R}\right)=\eta_{p} \eta_{a} \eta_{q} \eta_{c}
$$

The slope efficiency of the laser takes into account. $\eta_{p}$ is the pump efficiency, $\eta_{a}$ is the overlap efficiency, that takes into account that not all the population inversion may interact with the laser modes, $\eta_{q}$ is the quantum efficiency that accounts for the energy difference between the laser and pump photons and $\eta_{c}$ is the coupling efficiency of the resonator.

The slope efficiency allows us to express the output power as:

$$
P_{\text {out }}=\eta_{s}\left(P_{\text {pump }}-P_{\text {th }}\right)
$$


Plotting the output power as a function of pump power will provide the slope efficiency as the slope and the threshold power as the intersection of the curve with the abscissa axis.

The small signal gain $g$, operating with equation 3.22 and 3.28 , is given by:

$$
2 g L=\frac{2 \eta_{p} P_{\text {pump }}}{A_{\text {pump }} I_{\text {sat }, p}}
$$

And therefore the gain of the active medium is:

$$
G=\exp (g L)=\exp \left(\frac{\eta_{p} P_{\text {pump }}}{A_{\text {pump }} I_{\text {sat }, p}}\right)
$$

Another important parameter in the characterization of the laser is the resonator losses $\delta$ per roundtrip. Since the pump power at threshold is proportional to the small signal gain, operating with equations 3.22 and 3.28, we can express equation 3.19 as:

$$
-\ln R=\frac{2 \eta_{p}}{A_{p} I_{s a t, p}} P_{t h}-\delta
$$

Plotting the threshold power versus the logarithm of the output coupler reflectivity, provides the gain as the slope and the losses inside the cavity as the intersection of the abscissa. This method for measuring the loss inside the resonator was already proposed in [50].

The total energy provided by the laser diodes during the pumping time in QCW mode can be calculated as:

$$
E_{\text {diodes }}=N_{\text {diodes }} P_{\text {diodes }} f \Delta w
$$

where $N_{\text {diodes }}$ is the number of diode bars that pump the active medium, $P_{\text {diodes }}$ is the output power of an individual diode bar, $\Delta w$ is the temporal width of the current pulse that pumps the diodes and $f$ is the repetition rate of the laser.

With this theoretical knowledge we can fully characterize the performance of the pumping heads. In order to acquire all relevant, it is necessary to operate a PH5400 inside a resonator. 


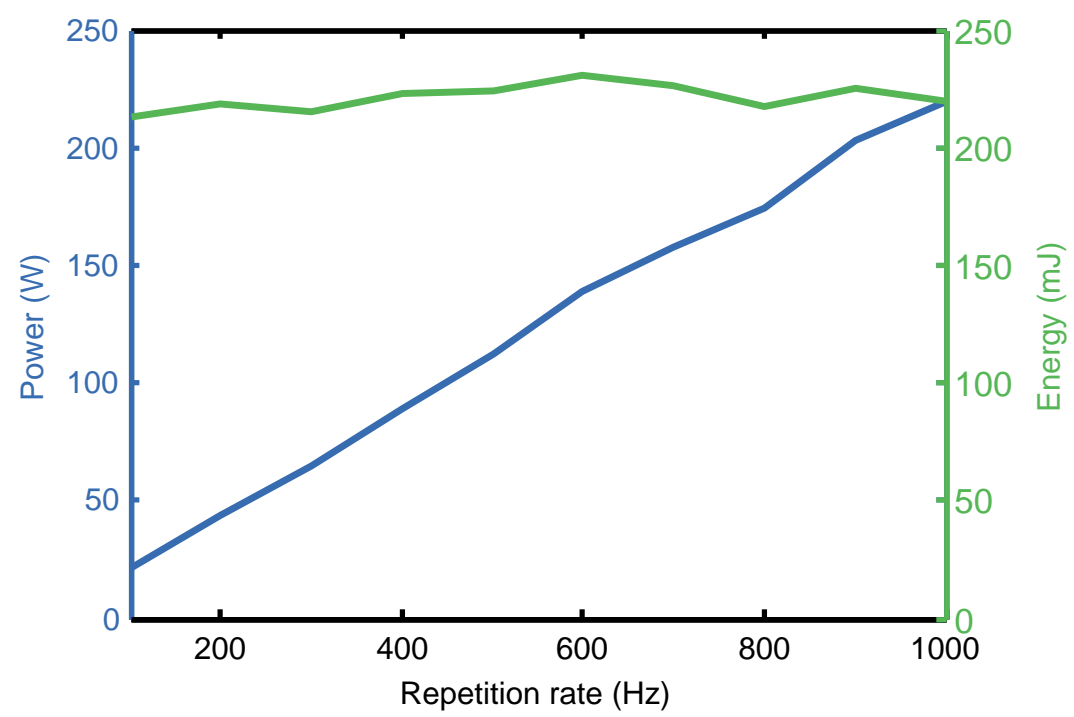

Figure 3.14: Blue line: Output power of a PH5400 pumping head hosting a $5 \mathrm{~mm}$ Nd:YAG rod working in QCW mode in a resonator with an output coupler of $85 \%$ as a function of the repetition rate. Green line: Energy per QCW pulse as a function of repetition rate.

\subsubsection{Nd:YAG QCW performance}

In order to fully characterize the performance of the pumping heads and measure all the important parameters described in the previous section, a PH5400 loaded with a Nd:YAG rod was operated in QCW mode.

For QCW operation we built a simple resonator with two plane-parallel cavity mirrors, the rear mirror had $99.9 \%$ reflectivity at the laser wavelength, while many mirrors with reflectivity between $20 \%$ and $85 \%$ were tested as the output coupler. The Nd:YAG rod was $5 \mathrm{~mm}$ diameter, $90 \mathrm{~mm}$ long and a doping concentration of $0.4 \%$ at. The separation between mirrors was $55 \mathrm{~cm}$.

The first step of the pumping heads as oscillator was to calibrate the behaviour as a function of the repetition rate. The input parameters were $220 \mu$ s square pulses and $100 \mathrm{~A}$ current for the whole range of working frequencies. The results can be seen in figure 3.14. Even though the repetition rate and thus the thermal load changes, the energy per pulse is constant. It is evident that the cavity conditions are changing as the thermal lens increases, so this means that all modes inside the cavity are being excited and contribute to a multimode beam.

A maximum energy of $212 \mathrm{~mJ}$ was achieved using $220 \mu \mathrm{s}$ long pump pulses, 


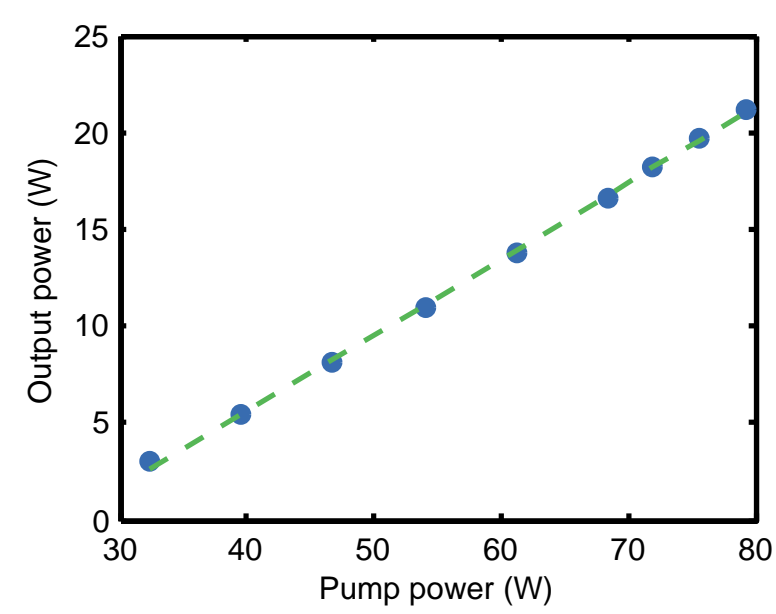

(a)

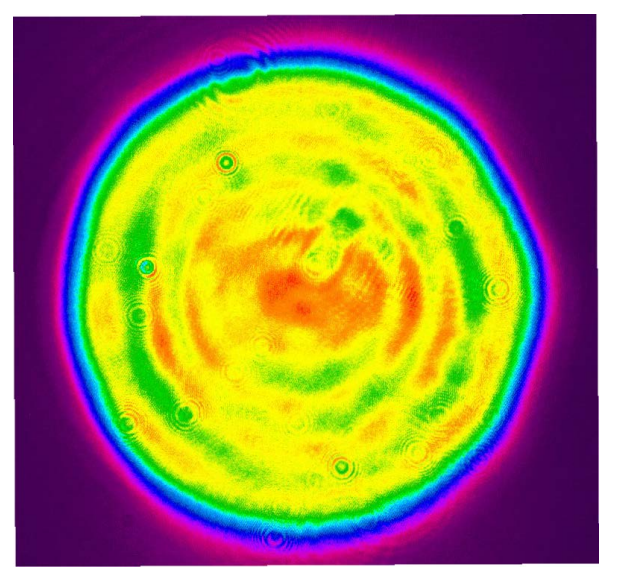

(b)

Figure 3.15: (a) Output power of a PH5400 pumping head loaded with a Nd:YLF working in QCW mode at a repetition rate of $100 \mathrm{~Hz}$ inside a resonator with an output coupler of $85 \%$ as a function of the input pump power of the laser diodes. Blue circular dots are experimental data points and the green dashed line is the tendency. (b) Near-field beam profile of the output of the resonator working at maximum power.

100 A of current, and an output coupler of $85 \%$ reflectivity, which was close to the optimum. The energy provided by the diodes in these conditions can be calculated according to equation 3.40 as $792 \mathrm{~mJ}$.

Figure 3.15 shows the behaviour of the output power as a function of the input power of the diodes. The fit of the experimental curve to a straight line shows that the slope efficiency of the laser is $39 \%$.

In order to calculate the roundtrip losses inside the resonator, $\delta$, we tested the pumping head in a resonator for several output couplers with different reflectivity. According to equation 3.39, plotting the threshold power versus the logarithm of the reflectivity, as in figure 3.16(a), the losses must be the cut of the fitted line with the reflectivity axis. The calculated value is $2.7 \%$.

The power as a function of reflectivity can be calculated according to equation 3.32 and is plotted in figure 3.16(b). The experimental points show a reasonable agreement with the theory.

The operation stability is another important parameter for the long-term operation of a laser. Many factors come into play in laser operation stability: the 


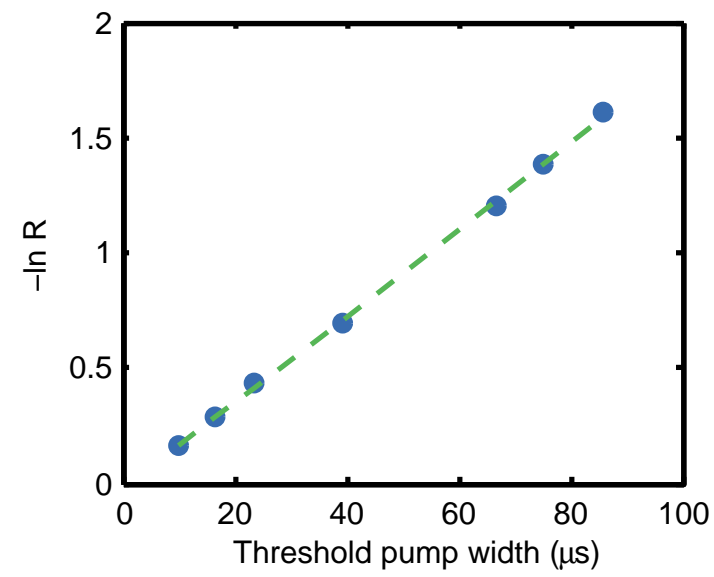

(a)

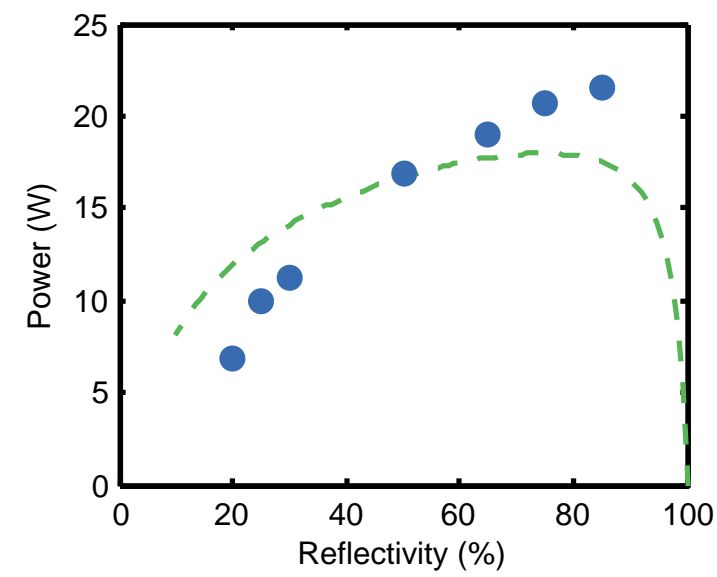

(b)

Figure 3.16: (a) Plot of the logarithm of reflectivity versus the threshold pump width for a pumping head with Nd:YAG. The linear fitting of this curve (green dashed line) allows us to derive the intracavity losses. (b) Plot of the experimental output power (blue dots) as a function of the reflectivity of the output coupler. The green dashed line represents the output power calculations (equation 3.32

roughness of the mechanical mounts of every component of the resonator, the cooling system and the pumping source. The use of diodes as opposed to flash lamps really enhances the stability of the laser, which can be as low as $1.2 \%$ rms.

\subsubsection{Nd:YLF QCW performance}

A similar procedure was applied for the characterization of the PH5400 with a Nd:YLF rod. A very simple resonator was built, with a concave mirror of 3000 mm radius of curvature and $99.9 \%$ reflectance while the output coupler was planeparallel of reflectivity in 20-85\% range. A test was performed with two plane-parallel mirrors but this kind of resonator proved to be unstable for Nd:YLF which confirms that the thermal lensing effect in Nd:YLF is weak. A thin film polarizer was added in order to tune the desired output wavelength. The rod inside the pumping head is a c-cut Nd:YLF rod with $5 \mathrm{~mm}$ diameter, $95 \mathrm{~mm}$ length and a doping concentration of $0.8 \%$ at.

The pumping heads with Nd:YLF were not tested above $200 \mathrm{~Hz}$, since there is a risk of fracture due to the strong thermal induced stresses and the YLF matrix is very brittle when compared to YAG. 


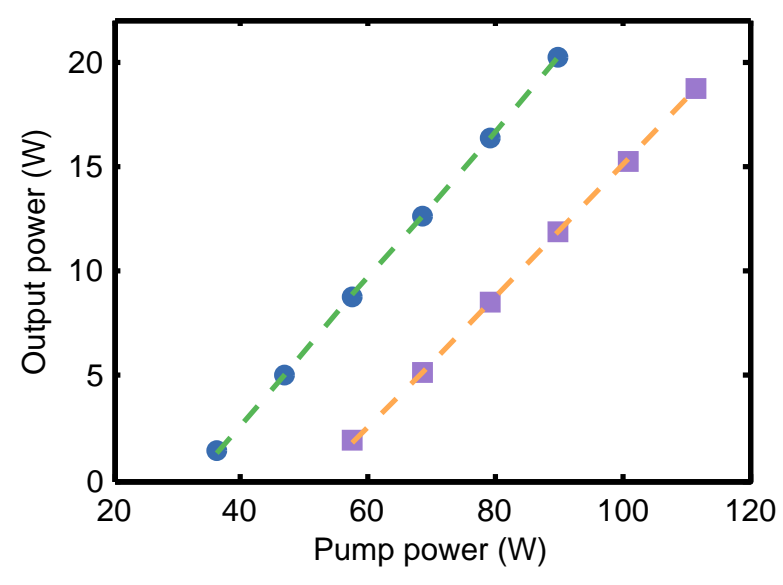

(a)

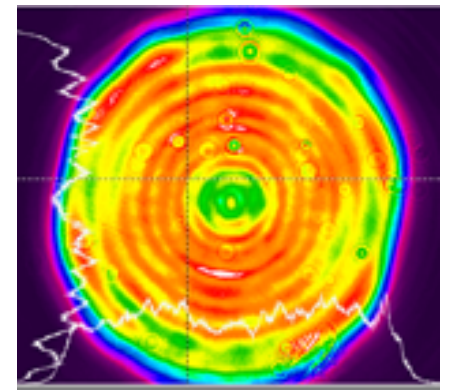

(b)

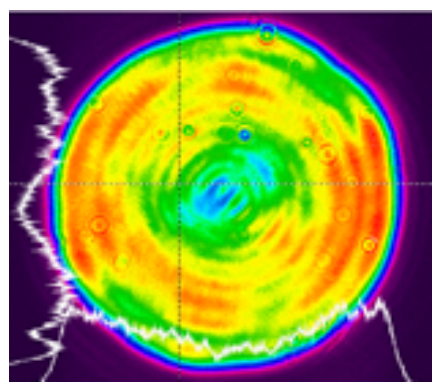

(c)

Figure 3.17: (a) Output power of a PH5400 pumping head loaded with a Nd:YLF working in QCW mode at a repetition rate of $100 \mathrm{~Hz}$ inside a resonator with an output coupler of $25 \%$ as a function of the input pump power of the laser diodes. Blue circular dots are experimental points for the $1047 \mathrm{~nm}$ wavelength and the green dashed line is the tendency. Purple square dots are experimental points for the $1053 \mathrm{~nm}$ wavelength and the orange dashed line is the tendency. (b) Near-field beam profile of the output of the resonator working at $1047 \mathrm{~nm}$. (c) Near-field beam profile of output of the resonator working at $1053 \mathrm{~nm}$.

The two polarizations behave quite differently in Nd:YLF due to their spectroscopic differences; the emission cross section of the $1047 \mathrm{~nm}$ wavelength is 1.5 times higher than that of the $1053 \mathrm{~nm}$, which translates in a lower saturation intensity and higher gain. Figure 3.17 illustrates the difference in the behaviour of the two polarizations in a resonator with an output coupler of $25 \%$ reflectivity. The slope efficiency is similar for both polarizations, 35\% and 32\% for the $1047 \mathrm{~nm}$ and 1053 $\mathrm{nm}$ respectively. The threshold pump power is higher for the $1053 \mathrm{~nm}$ polarization.

The near field beam profile of $1047 \mathrm{~nm}$ (figure 3.17(b)) and $1053 \mathrm{~nm}$ (figure $3.17(\mathrm{c})$ ) are both very similar to the output of the one using Nd:YAG, producing a very multimode output. The beam profile of $1053 \mathrm{~nm}$ has lower fluence around its centre, which can be due to the lower gain of this wavelength and a suppression of 


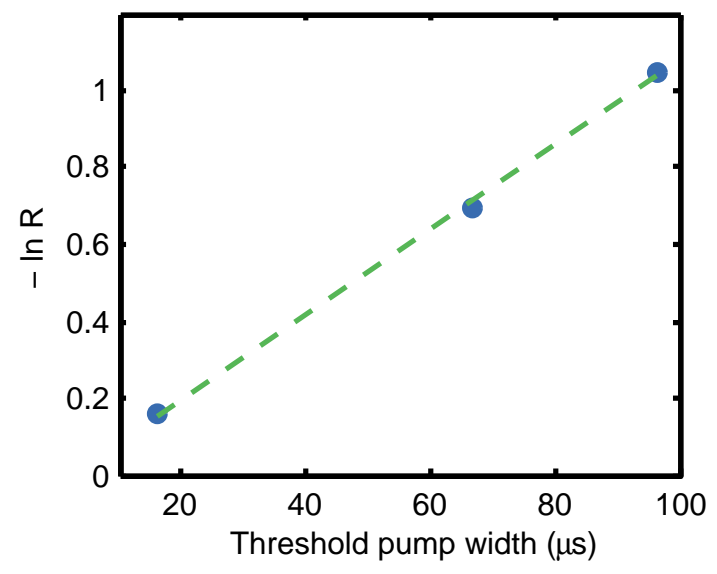

(a)

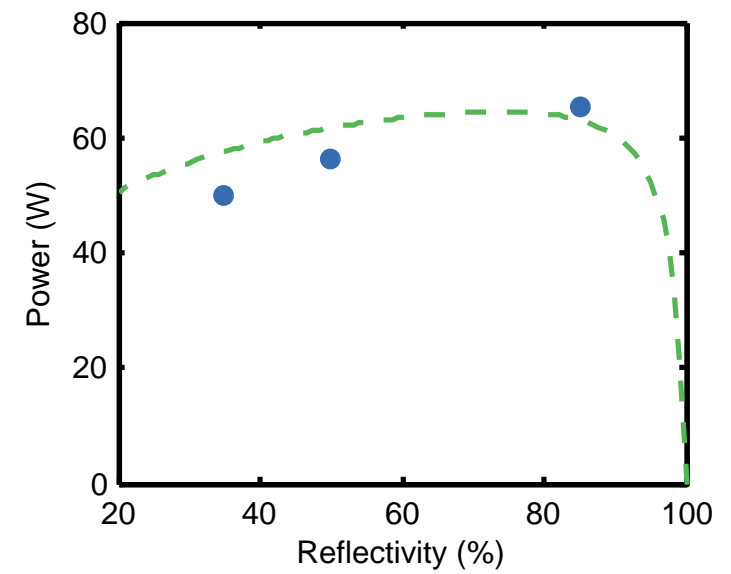

(b)

Figure 3.18: (a) Plot of the logarithm of reflectivity versus the threshold pump width for a pumping head with Nd:YLF working at $1047 \mathrm{~nm}$. The linear fitting of this curve (green dashed line) allows as to derive the intracavity losses. (b) Plot of the experimental output power (blue dots) as a function of the reflectivity of the output coupler. The green dashed line represents the output power calculations (equation 3.32

modes by the intracavity polarizer. This feature would fade if the curvature of the rear mirror was changed.

Although a polarizer is present in the cavity, the losses inside the resonator should be similar to the Nd:YAG. The same procedure of plotting the threshold pump width versus the logarithm of reflectivity was applied as in figure 3.18(a). The rountrip loss was found to be $2 \%$.

The highest output energy that was achieved working at $100 \mathrm{~Hz}$ and $1047 \mathrm{~nm}$ was $650 \mathrm{~mJ}$, for $500 \mu \mathrm{s}$ and 100 A pump pulses in a resonator with a $85 \%$ reflectivity output coupler. The behaviour is illustrated in figure 3.18(b). 


\section{Chapter 4}

\section{Development of a nanosecond}

\section{laser}

The use of the PH5400 pumping heads in QCW mode is not enough to build a suitable pump laser for a femtosecond Ti:Sa CPA chain, due to the high pulse duration and low energy per pulse. The Ti:Sa crystal has a fluorescence lifetime of $3.3 \mu \mathrm{s}$ and, thus, the pump pulse duration must be much lower than this value or the crystal would lose most of the pump energy by spontaneous emission.

In order to obtain a pulse with a duration lower than microseconds from a resonator with a pumping head, we can use a technique known as Q-switching. Q-switching is achieved by inserting an element that introduces losses inside the cavity, which decreases the $\mathrm{Q}$ factor of the resonator. This element can be either active, when the losses are modulated by some controllable element, usually an electro-optic or acousto-optic modulator, or passive, when the losses are modulated automatically by a saturable absorber, an opaque material that becomes transparent when the intensity of light exceeds some threshold. The energy per pulse can be as high as the stored energy in the active medium, ranging from a few microjoules to hundreds of milijoules, while the pulse duration is typically in the nanosecond range; the pulse duration is determined by the gain of the active medium and by the round-trip time, which depends on the length of the resonator. This technique allows the production of short pulses with high energy, reaching GW peak powers.

The development of a nanosecond source for pumping the Ti:Sa involves high energy that cannot be achieved with a single Nd:YAG or Nd:YLF pumping head. In 
order to further amplify the energy of the oscillator, the most common architecture for nanosecond lasers is called MOPA which stands for Master Oscillator Power Amplifier. A MOPA laser consists of a seed laser, the oscillator, and one or more optical amplifiers that boost the output power while preserving some fundamental properties of the oscillator like wavelength, coherence, etc. Such an architecture also offers the advantage of power scalability but it usually requires increasing the size of the laser crystals and more powerful pump sources.

This chapter will cover the development of high average power pulsed MOPAs using PH5400 pumping heads as oscillator and amplifiers, to serve as pumping lasers for a Ti:Sa CPA laser.

\subsection{Diode Pumping heads: Q-switching perfor- mance}

Both Nd:YAG and Nd:YLF are materials that can store large fluences and thus are good candidates for high power Q-switch operation. In order to evaluate the performance of the Q-switched Nd:YAG and Nd:YLF it is best to have a theoretical understanding of this process. The active medium is pumped but stimulated emission amplification is suppressed by the low Q factor of the resonator; once the pumping of the active medium has ended, the Q factor of the cavity is switched and rises steeply generating a circulating photon flux that depletes the energy stored in a few roundtrips, emitting a short and energetic pulse. Q-switching is well known since the early times of lasers, but with advances in technology this technique has become robust and reliable as the switching of the cavity can be very fast and efficient.

\subsubsection{Q-switch theory}

Q-switching is by definition a dynamic process and thus its description is ruled by the transient behaviour of intensity and population inversion. A model for the behaviour of the laser during Q-switching can be extracted from the rate equations (see chapter 3: 


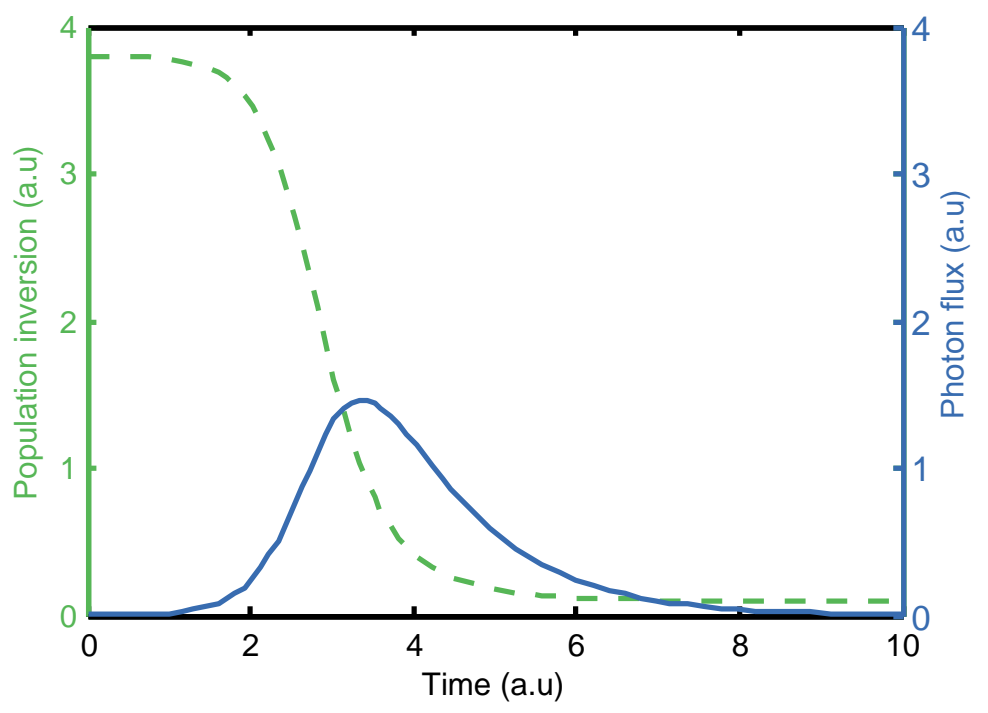

Figure 4.1: Simulation of the dynamic behaviour of population inversion and photon flux during Q-switching.

$$
\begin{array}{r}
\frac{d N}{d t}=-\frac{\sigma c}{V} \phi N-\frac{N}{\tau_{f}} \\
\frac{d \phi}{d t}=\sigma c \phi N-\frac{\phi}{\tau_{c}}
\end{array}
$$

The initial moment $t=0$ is considered when the active medium has reached a population inversion $N_{0}$ and the pumping source is cut off, $\Gamma_{p}=0$ (already eliminated from the equations above). The Q-switching losses are introduced in the photon cavity lifetime as a time dependent term $Q(t)$ :

$$
\tau_{c}=\frac{2 L}{c} \frac{1}{\delta-\ln R+Q(t)}
$$

The system of differential equations 4.1 and 4.2 does not have an analytical solution, and must be solved numerically in order to obtain a complete dynamic description of the pulse build-up during Q-switch, for which a Matlab script was used. Figure 4.1 is an example of the dynamics of intensity and population inversion during Q-switching action; the population inversion decreases steeply as the flux rises and falls when the energy available is depleted.

While the set of equations 4.1 and 4.2 cannot originate a closed-form expression, 
quantitative solutions for optimal Q-switch parameters were derived by Degnan et al. [51]. These parameters can be expressed as a function of a dimensionless term $z$ :

$$
z=\frac{2 g L}{\delta}
$$

Which is essentially the ratio between the rountrip gain of the active medium and the losses. The optimum reflectivity of the output coupler for maximum energy extraction can be formulated as:

$$
R_{\text {opt }}=\exp \left(-\delta\left(\frac{z-1-\ln z}{\ln z}\right)\right)
$$

The optimum reflectivity is a function that decreases with increasing $z$. Therefore a laser with a high gain to loss ratio should include a low reflectivity output coupler in order to maximize the output energy, in agreement with equation 4.5 for a QCW laser.

The maximum energy that can be extracted during Q-switch is:

$$
E_{\max }=\frac{A \delta h \nu}{2 \sigma}(z-1-\ln z)
$$

This function tends to the total available energy for large $z$ :

$$
E_{\max , z \rightarrow \infty}=V h \nu N
$$

Therefore, in order to maximize the extraction efficiency and output energy of the Q-switch, two actions can be performed: increasing the gain by rising the energy density in the active medium or decreasing the rountrip losses.

The energy stored in the active medium before the Q-switch is activated depends on the temporal shape of the pump pulse and the fluorescence lifetime: when the Q-switching is off, the pump rate increases the stored energy while the spontaneous emission tends to decrease it. The maximum stored energy is reached the moment the pump pulse ends. In the case of the PH5400 pumping heads, the laser diode pulse can be represented by a rectangular function.

Finally, the FWHM pulse duration can be expressed as: 


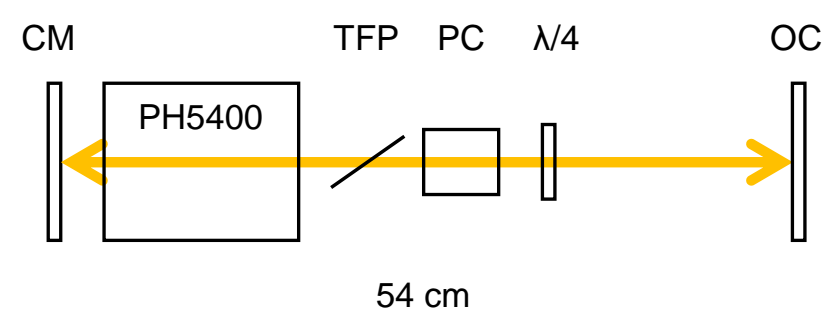

Figure 4.2: Experimental setup of the electro-optic Q-switched laser. CM is the curved rear mirror and OC the output coupler. PH5400 is the pumping head, PC is a Pockels cell and TFP represents the thin film polarizer that creates a low $\mathrm{Q}$ resonator along with the quarter waveplate $\lambda / 4$.

$$
t_{p}=\frac{2 L}{\delta c} \frac{\ln z}{z} \frac{1}{1-\frac{z-1}{\ln z}\left(1+\ln \frac{z \ln z}{z-1}\right)}
$$

The pulse duration is shorter for large $z$. It makes sense that the higher the gain or lower the losses, the pulse will take less roundtrips to extract the energy from the active medium.

These equations provide valuable information for the design of a Q-switched laser and can work as a benchmark for its performance.

\subsubsection{Nd:YAG Q-switching performance}

In order to prove Q-switching lasing with a PH5400, we built a resonator using a Pockels cell for switching the Q factor of the cavity. The experimental setup of the Q-switched laser is shown in figure 4.2. The crystal used is a $100 \mathrm{~mm}$ long, $5 \mathrm{~mm}$ diameter Nd:YAG rod doped $0.3 \%$ at. The rear mirror of the resonator is a high reflectivity curved mirror of $5000 \mathrm{~mm}$ radius of curvature (ROC), while the output coupler is a plane-parallel $30 \%$ reflectivity mirror.

The electro-optic active Q-switching used a Pockels cell powered by $3.4 \mathrm{kV}$ for quarter waveplate operation, for which a high voltage driver is needed. The Pockels cell enclosure must be properly designed in order to avoid electromagnetic interference to other equipment due the noise radiated by the high voltage switch. The Pockels cell is made of $\mathrm{KD}^{*} \mathrm{P}$, a material which is inexpensive and works well 


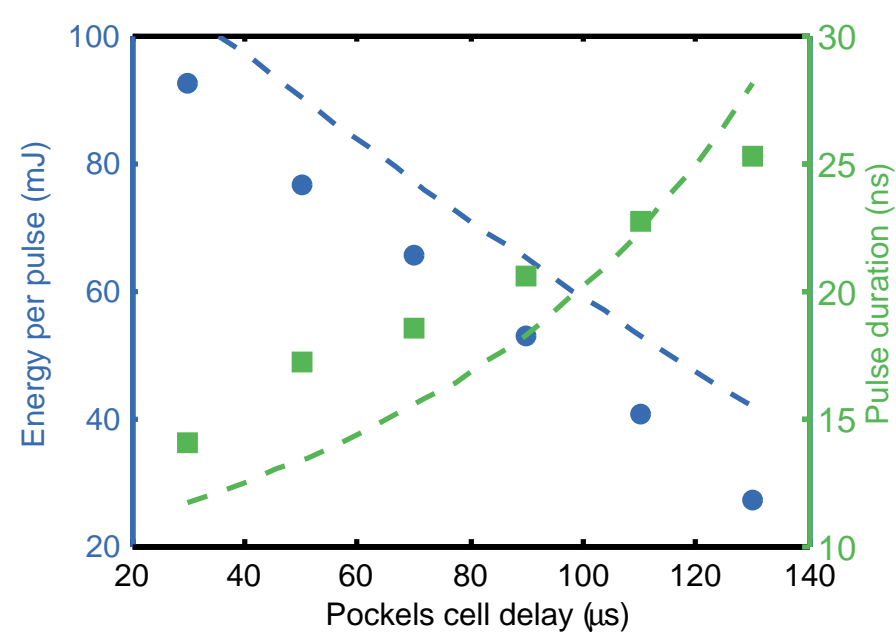

(a)

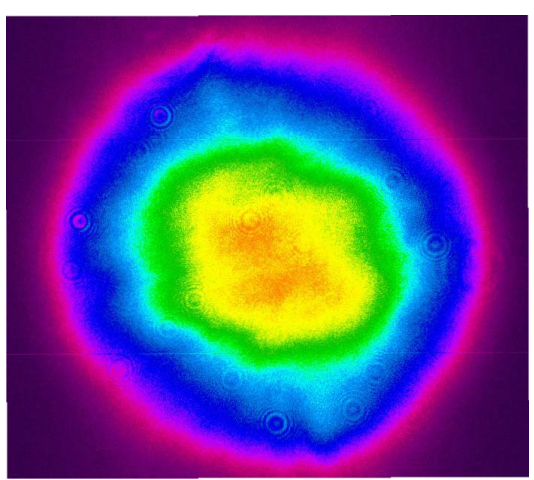

(b)

Figure 4.3: (a) Performance of the output energy per pulse (blue) and pulse duration (green) as a function of the Pockels cell delay. The dashed line represent the Q-switch simulations. (b) Near-field beam profile of the output beam. Its diameter is $3.8 \mathrm{~mm}$.

in the $100 \mathrm{~Hz}$ frequency range, but can introduce unwanted losses and induce deformation of the phase front.

The energy stored in the Nd:YAG rod can be calculated for a pump pulse width of $200 \mu$ s and $100 \mathrm{~A}$ is $293 \mathrm{~mJ}$. The energy of the Q-switched laser is controlled through the delay of the Pockels cell. The delay is the time difference between the falling signal edge of the pump pulse and the rising edge of the Pockels cell trigger. This delay lowers the stored energy in the active medium but more importantly it does not affect the thermal equilibrium of the laser. Therefore, it was the preferred method for changing the output energy per pulse of the Q-switched laser, as opposed to varying the input pump power of the diodes, which would also affect the thermal load of the Nd:YAG.

A maximum output energy of $105 \mathrm{~mJ}$ and $12 \mathrm{~ns}$ pulse duration at the minimum delay possible was obtained from the Q-switched laser with the parameters described above. The behaviour of the output energy per pulse and pulse duration versus delay is presented in figure 4.3(a). The dashed lines represent the simulations performed with the parameters of the Nd:YAG rod and the resonator. A good agreement between the experimental data and the calculations can be observed, 
in particular for the energy per pulse. The pulse duration values do not agree so well with the simulations because of spatio-temporal mode dynamics. For high values of Pockels cell delay, the gain experienced by the laser is low and the output spatial mode is close to a single mode, but the increase of gain when the Pockels cell delay tends to a minimum excites higher order modes and the beam profile at maximum power is far from a single mode (figure 4.3(b)). A consequence of this dynamic is the appearance ripples in the temporal profile of the pulse, known as mode beatings, caused by fluctuations of the phase between the different resonator modes.

The near field beam profile in figure 4.3(b) is an incoherent superposition of many high order modes of the cavity that can coexist thanks to the high rountrip gain and large area of the Nd:YAG rod. The beam has a diameter of $3.8 \mathrm{~mm}$ measured at a short distance of the output coupler, where the beam waist is located.

The size of the beam waist $\left(w_{0}\right)$ for a gaussian beam in a cavity, where one of the mirrors is plane-parallel (the output coupler), can be calculated according to [52] as:

$$
\begin{aligned}
w_{0} & =\frac{\lambda L}{\pi}\left(\frac{g_{M}}{1-g_{M}}\right) \\
w_{M} & =\frac{\lambda L}{\pi}\left(\frac{1}{g_{M}\left(1-g_{M}\right)}\right)
\end{aligned}
$$

where $g_{M}$ is an adimensional parameter that takes into account the length of the resonator $L$ and the radius of curvature of the mirror $r,\left(g_{M}=1-\frac{L}{r}\right)$, and is equal to one in the case of a plane-parallel mirror. This parameter rules the stability of the resonator, and in this case must be less than one for it to be stable.

$w_{M}$ in equation 4.10 is the beam size in the curved mirror, which is the maximum size the beam can reach in a resonator with a curved and a plane mirror. That is the reason why the pumping head was located as close as possible to the rear mirror, CM in figure 4.2.

For a resonator like the one pictured in figure 4.2, with a distance of $54 \mathrm{~cm}$ between mirrors and $3000 \mathrm{~mm}$ ROC rear mirror, the size of a gaussian beam is 0.53 $\mathrm{mm}$ at the curved mirror and $0.46 \mathrm{~mm}$ at the waist, assuming a negligible thermal lens. This means that the overlap of the fundamental mode with the available 


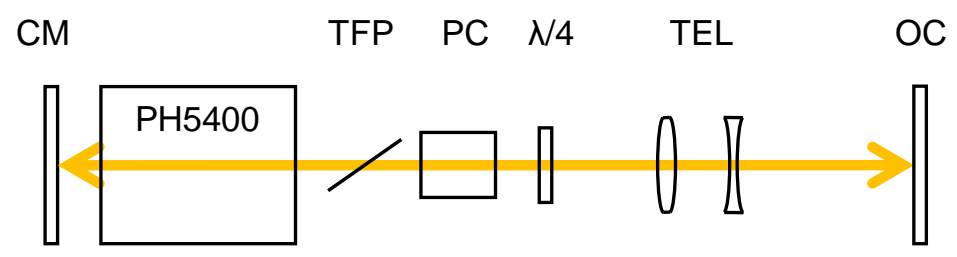

$65 \mathrm{~cm}$

Figure 4.4: Experimental setup of the electro-optic Q-switched laser. CM is the curved rear mirror and OC the output coupler. PH5400 is the pumping head, PC is a Pockels cell and TFP represents the thin film polarizer that creates a low Q resonator along with the quarter waveplate $\lambda / 4$. TEL is a Galilean telescope comprised of a positive and negative lens.

pumped area of the rod is about $5 \%$ and high order modes will dominate.

In high power lasers it is usual that the beam profile is multimode, a feature that is sometimes looked after specially in the case of pump lasers, where a flat top beam is preferred to a gaussian due to uniformity. However, for other applications a multimode beam is undesired, due to their bad coherence and far-field properties, and it is necessary to obtain a single gaussian transversal mode from the resonator.

There are several methods of forcing a single transversal mode from a high power Q-switched resonator. A possible solution is the use of an unstable resonator combined with a mirror with radially variable reflectivity, called gaussian mirrors. Another alternative is the use of a larger resonator, that according to equation 4.10 will increase the fundamental mode size near the position of the active medium. The inconvenience is that enlarging the cavity also increases the pulse duration and creates more optical and mechanical instability. A more suitable solution can be the use of an intracavity telescope [53]. The insertion of an intracavity telescope virtually enlarges the resonator which results in larger beam sizes in the laser rod while preserving a short and stable resonator because the telescope can be adjusted to compensate for the thermal lensing.

A Galilean telescope is comprised of two lenses: a concave lens of focal length $f_{2}$ and a convex lens of focal length $f_{1}$. The magnification of the telescope can be expressed as: 


$$
M=-\frac{f_{2}}{f_{1}}
$$

A telescope is said to be collimated when the distance between lenses is $f_{2}-\left|f_{1}\right|$. However, if a small variation of the distance between the lenses is added, $\Delta$, the telescope behaves like a positive lens with a focal length of:

$$
f_{T}=\frac{f_{2}^{2}}{\Delta}
$$

The combination of the lenses created by thermal lensing, the defocusing of the telescope and the curvature of the rear mirror create an equivalent lens that has the ability to modify completely the behaviour of the resonator. It can be shown that if $g_{M}=0.5$, the resonator is inside the region of stability and is less sensible to variations of $g_{M}$. Thermal lensing fluctuations can induce changes in $g_{M}$, hence it is advisable to operate in this configuration, which will be referred to as well adapted resonator.

In order to have a well adapted resonator, if we assume that the curved mirror, the Nd:YAG rod and the telescope are close together, the equivalent lens must fulfil the condition:

$$
\frac{1}{2 M^{2} L}=\frac{1}{f_{T}}+\frac{1}{f_{t h}}+\frac{2}{r}
$$

where $f_{t h}$ is the focal length of the active medium and $r$ is the ROC of the curved mirror. The spot sizes for a well adapted resonator are:

$$
\begin{aligned}
w_{0} & =\sqrt{\frac{\lambda L}{\pi}} \\
w_{M} & =\sqrt{\frac{2 \lambda L}{\pi}} M
\end{aligned}
$$

Note that the beam waist at the output coupler in this configuration (equation 4.14 ) is independent of the magnification of the telescope used and only depends on the length of the resonator. This fact imposes a limit to the output fluence of the oscillator before risking optical damage.

In order to design the telescope associated to the resonator one must choose a value for the waist of the beam inside the gain medium. It is limited not only by 


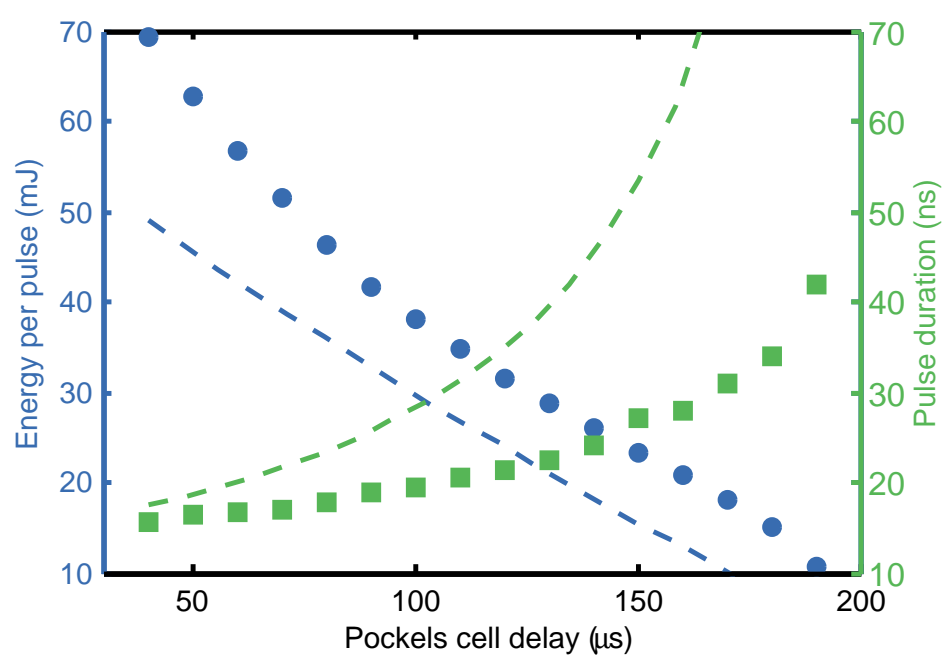

(a)
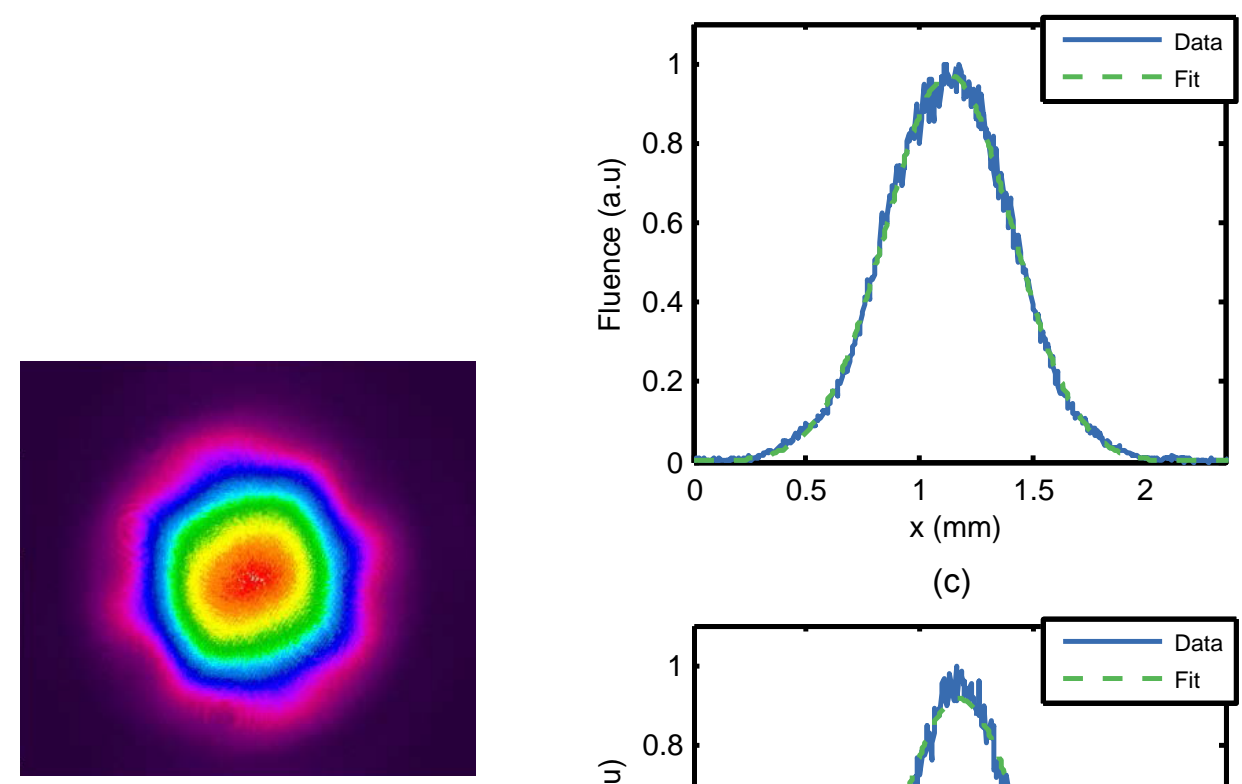

(c)

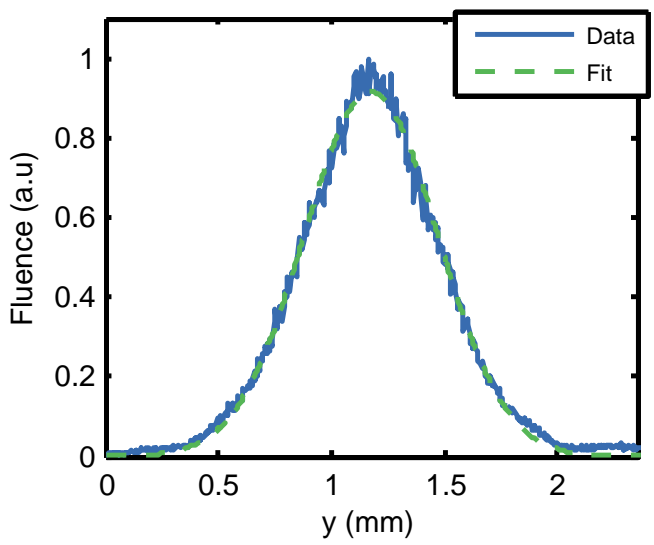

(d)

Figure 4.5: (a) Performance of the electro-optic Q-switched Nd:YAG laser with an intracavity telescope. (b) Near-field beam profile of the output beam. Its diameter is $1.2 \mathrm{~mm}$. (c) and (d) are one dimensional cuts of the beam profile (straight line) and their gaussian fit (dashed line). 
the physical size of the rod but also by its optical quality and distribution of the pump energy and other optical distortions that may affect the beam mode inside the resonator.

The intracavity telescope was chosen as a combination of a positive lens with a focal length of $100 \mathrm{~mm}$ and the negative lens with a focal length of $30 \mathrm{~mm}$, which provide a magnification of $M=3.4$ while the defocus needed to fulfil the well adapted resonator condition was measured to be $11.3 \mathrm{~mm}$. The telescope was placed between the output coupler and the Pockels cell because we feared that the small size of the beam between the telescope and the output coupler could damage any optical element situated in that position. Employing equation 4.15 the theoretical value of the beam in the position of the Nd:YLF rod is $4.3 \mathrm{~mm}$ which now covers $74 \%$ of the area for the same diameter rod.

The performance of a resonator with an intracavity telescope with Nd:YAG can be observed in figure 4.5(a). The maximum output energy achieved was $70 \mathrm{~mJ}$; the delay of the Pockels cell trigger could be reduced to extract more energy but damage was often observed in the output coupler if the output energy was higher. This can be explained by the size of the beam near the output mirror, which was $1.2 \mathrm{~mm}$ (figure 4.5(b)), close to the theoretical value of $1 \mathrm{~mm}$ returned by equation 4.14. The peak fluence of a gaussian beam with that size and energy is close to 18 $\mathrm{J} / \mathrm{cm}^{2}$, well beyond the damage threshold for nanosecond pulses provided by the manufacturer of $10 \mathrm{~J} / \mathrm{cm}^{2}$.

Increasing the repetition rate of the laser beyond $200 \mathrm{~Hz}$ leads to greater deviations from the gaussian mode due to the fact that the focal length of the thermal lensing grows and defocusing the intracavity telescope may not be enough. Therefore another telescope setup is needed to compensate for the additional deviations at very high frequency.

\subsubsection{Nd:YLF Q-switching performance}

Nd:YLF is also a good material for Q-switching, as it has a fluorescence lifetime almost 3 times larger than Nd:YAG and can generate more energetic pulses. The experimental setup of the Nd:YLF Q-switching laser is similar to the one shown in Fig. 4.2 for Nd:YAG. The crystal used is a $100 \mathrm{~mm}$ long, $5 \mathrm{~mm}$ diameter Nd:YLF 


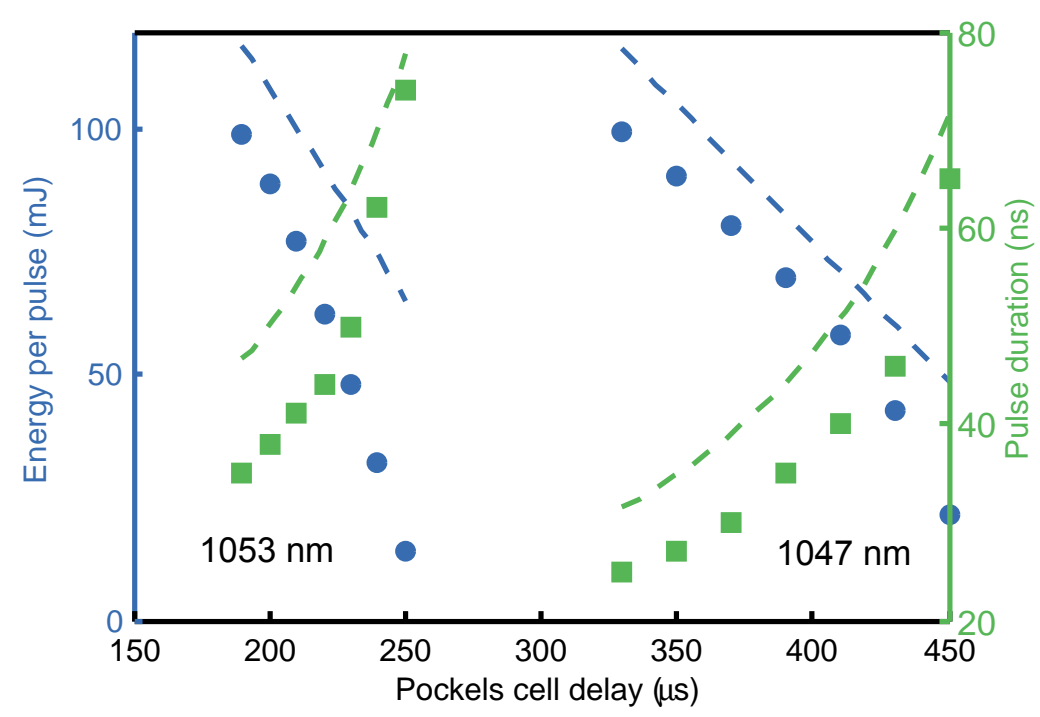

Figure 4.6: Performance of the electro-optic Q-switched Nd:YLF laser for the two wavelength modes.

rod doped $0.8 \%$ at. The rear mirror of the resonator is a curve reflective mirror $(>99.9 \%)$ and the output coupler is a $30 \%$ reflectivity plane-parallel mirror. The electro-optic Q-switch was accomplished using a KD*P Pockels cell together with a TFP and a quarter waveplate. The pumping pulse length can reach $500 \mu \mathrm{s}, 100$ A square pulses, which represents a $5 \%$ duty.

The performance of the Q-switched laser was investigated for both wavelengths using the same resonator, just by rotating $90^{\circ}$ the orientation of the intracavity polarizer while the repetition rate was fixed at $100 \mathrm{~Hz}$. Figure 4.6 displays the energy per pulse versus the delay of the Pockels cell. The $1047 \mathrm{~nm}$ wavelength has a higher emission cross section and therefore a lower threshold, while the $1053 \mathrm{~nm}$ wavelength requires a higher gain in the active medium to start. The simulations are in good agreement with the experimental data in the case of the $1047 \mathrm{~nm}$ wavelength. However in the case of $1053 \mathrm{~nm}$ wavelength, the slope is steeper but the simulations do not match adequately to the experiment. This can be due to the inaccuracy on the measured values of some parameters like the saturation fluence for this YLF rod and polarization.

The output beam of the Nd:YLF oscillator is multimode similar to the Nd:YAG beam. In the same way as for Nd:YAG, we built a resonator with an intracavity telescope. The design of the telescope is easier because the thermal lensing effect 
of YLF can be neglected.

The performance of a resonator with an intracavity telescope with Nd:YLF can be observed in figure 4.7(a). The intracavity telescope consisted of a positive lens with a focal length $125 \mathrm{~mm}$ and the negative lens had a focal length of $30 \mathrm{~mm}$, a magnification of $M=4.2$. Employing eq. 4.15, the theoretical value of the beam in the position of the Nd:YLF rod is $5.4 \mathrm{~mm}$, which covers entirely the rod and. An output energy per pulse of $58 \mathrm{~mJ}$ was achieved for a Pockels cell delay of $100 \mu \mathrm{s}$. The simulated pulse duration lines pretty well to the experimental data but the energy per pulse is overestimated in the calculations probably due to discrepancies between the theoretical and real beam size at the active medium.

The diameter of the beam near the output mirror was $1.04 \mathrm{~mm}$, pictured in figure 4.7(b), which represents a peak fluence of $14 \mathrm{~J} / \mathrm{cm}^{2}$. The theoretical value of the beam is $0.92 \mathrm{~mm}$ according to equation 4.14. A beam this size would cause optical damage in the output mirror with higher energy per pulse as the damage threshold provided by the manufacturer is $10 \mathrm{~J} / \mathrm{cm}^{2}$ for $10 \mathrm{~ns}$ pulses. Figures 4.7(c) and 4.7(d) represent horizontal and vertical cuts of the beam compared to a gaussian fit of the beam profile. They are not completely gaussian since the peaks are slightly flattened, probably because the magnification of the telescope is too high and the beam is clipped at the rod.

The low energy per pulse achieved in gaussian mode operation does not take advantage of the full potential of Nd:YLF. One of the most important properties of this material is the large amount of energy that can support and would make a PH5400 with this crystal inefficient functioning as an oscillator.

\subsection{Nanosecond Nd:YAG MOPA}

In order to deliver larger energy per pulse an arrangement of PH5400 pumping heads as amplifiers was used to boost the energy output of the Q-switched oscillator. We built a Nd:YAG MOPA using 8 PH5400 pumping heads, two of them working in the oscillator and six of them as amplifiers.

The laser output was stable in long term operation with a variation of $\approx 2.1 \%$ rms after two consecutive hours at maximum power. No degradation of power or loss of conversion efficiency was observed during the time the laser worked in the 


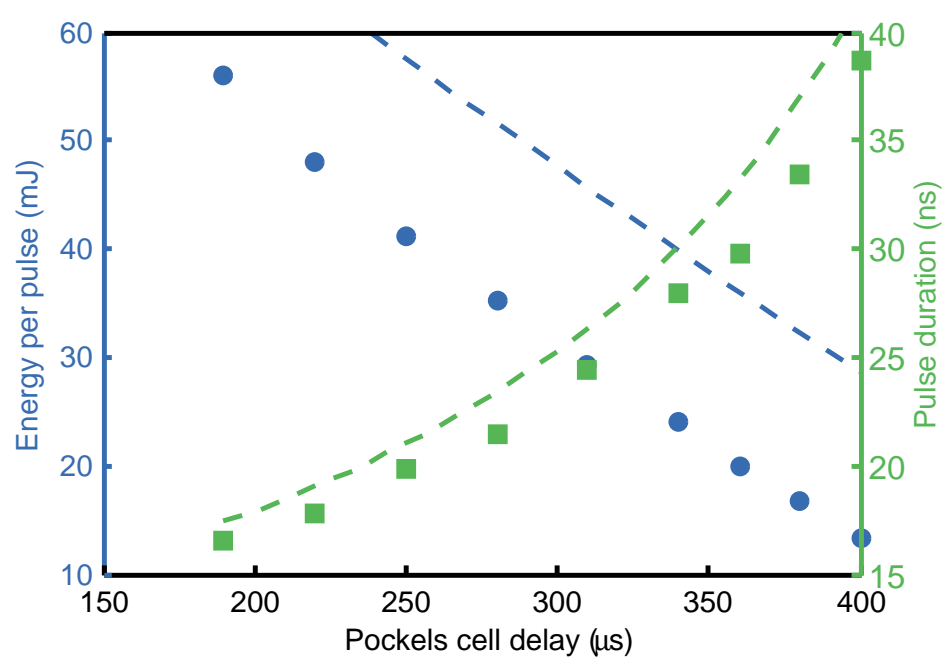

(a)

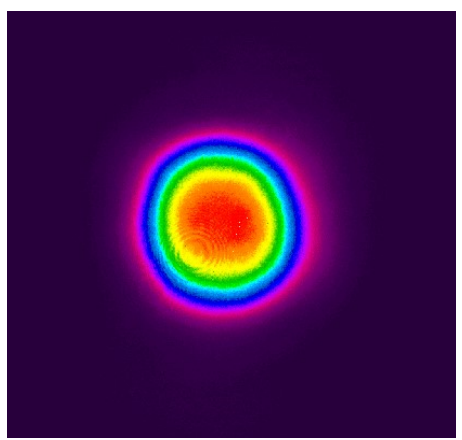

(b)

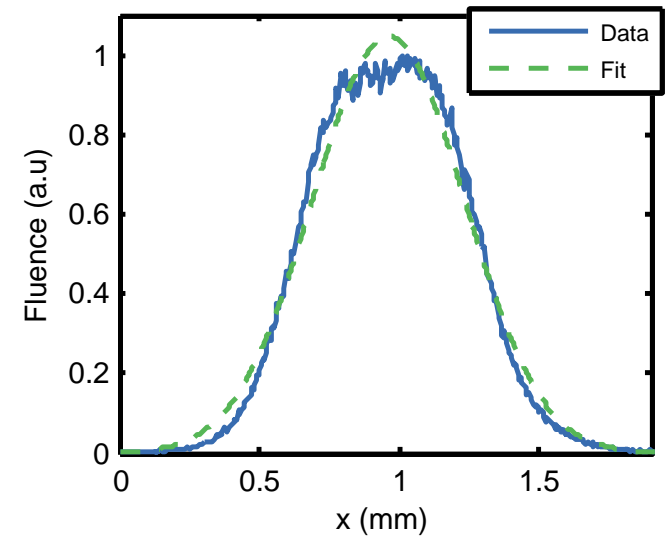

(c)

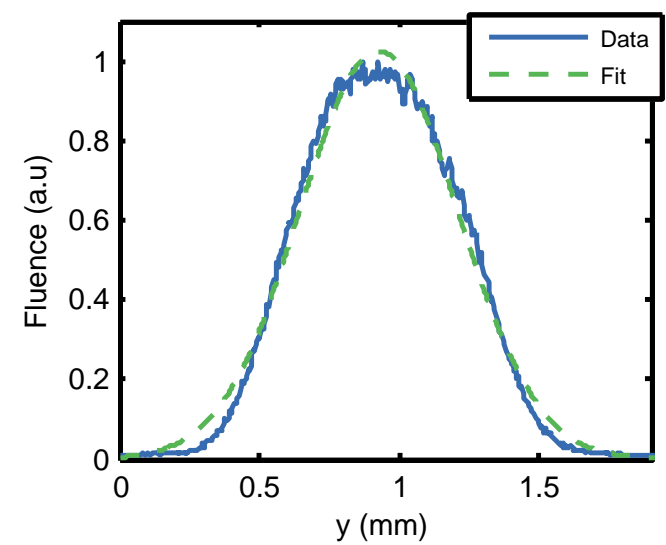

(d)

Figure 4.7: (a) Performance of the electro-optic Q-switched Nd:YLF laser with an intracavity telescope. (b) Near-field beam profile of the output beam. Its diameter is $1.04 \mathrm{~mm}$. (c) and (d) are one dimensional cuts of the beam profile (straight line) and their gaussian fit (dashed line). 
laboratory.

The final specifications of this laser are $391 \mathrm{~mJ}$ total energy per pulse divided into two beams of $\approx 200 \mathrm{~mJ}$ each and $10 \mathrm{~ns}$ pulse duration, working at $100 \mathrm{~Hz}$ repetition rate and $532 \mathrm{~nm}$.

\subsection{Nanosecond Nd:YLF MOPA}

Nd:YLF has some advantages with respect to Nd:YAG, including higher stored energy and less thermal lensing optical power. For a MOPA these properties mean more gain in the amplifiers and less restrictions due to thermal management. It consists of an oscillator with a single pumping head and two pairs of amplifiers for a total of five PH5400.

The final specifications of this laser are $391 \mathrm{~mJ}$ total energy per pulse divided into two beams of $250 \mathrm{~mJ}$ each and210 ns pulse duration, working at $100 \mathrm{~Hz}$ repetition rate and $527 \mathrm{~nm}$.

Below, table 4.1 summarizes the parameters of the two nanosecond lasers built for pumping the Ti:Sa. The Nd:YLF MOPA has higher output energy and efficiency compared to the Nd:YAG MOPA even though the first included three PH5400 pumping heads less. However, one potential advantage of the Nd:YAG MOPA is the higher repetition rate capability. Nevertheless, for the radioisotope generation, which is the main goal of this thesis, it is difficult to work at repetition rates higher than $100 \mathrm{~Hz}$, due to limitations in thin foil target refreshing and refocusing at higher speeds. 


\begin{tabular}{lll}
\hline & MOPA 1 & MOPA 2 \\
\hline Active material & Nd:YAG & Nd:YLF \\
Number of pumping heads & 8 & 5 \\
Wavelength (nm) & 532 & 527 \\
Repetition rate (Hz) & 100 & 100 \\
Total energy (mJ) & 400 & 500 \\
Pulse duration (ns) & 11 & 19 \\
\hline
\end{tabular}

Table 4.1: Summary of parameters of Nd:YAG and Nd:YLF MOPAs. 


\section{Chapter 5}

\section{Development of an ultrashort}

\section{pulse laser}

During the development of short pulse laser sources and high energy lasers, a bottleneck in amplification was formed because of the high intensity of these pulses which caused non-linear effects which led to optical damage in the bulk of materials. Then the advent of chirped pulse amplification (CPA) [6] and the discovery of Titanium:Sapphire and Kerr lens mode-locking [4] as a suitable source of femtosecond-level pulses opened the door to the field of short pulse technology. Although other materials have been growing in popularity through the last decade for the generation of sub picosecond pulses, like Yb doped materials, which have the advantage of a longer lifetime so they can store more energy and the availability of high power laser diodes as a suitable pump source, Titanium:Sapphire is still the go-to material to construct ultra-high power lasers.

In this work we decided to use Ti:Sa to build a CPA TW-class high average power laser and this chapter will detail its setup and construction. The two nanosecond MOPAs developed in the previous chapter were designed to be used to pump this material and play a fundamental role to achieve a good performance at high repetition rates. 


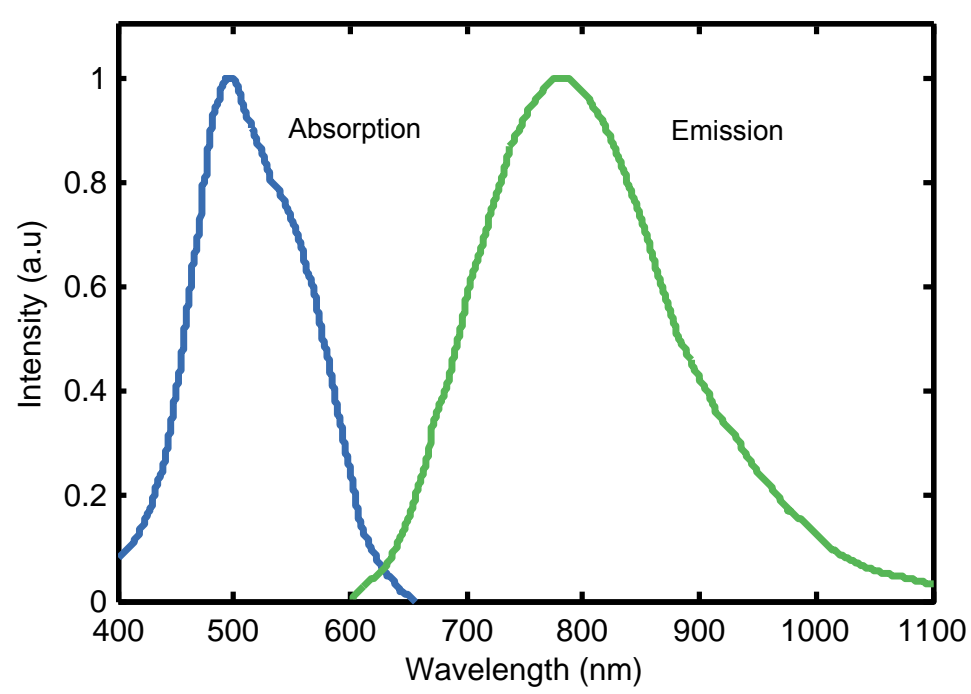

Figure 5.1: Absorption and emission cross sections of the $\pi$ polarization of Ti:Sa.

\subsection{Titanium:Sapphire}

The study of Titanium:Sapphire began in the 80s when Moulton achieved the first lasing action using this material [54]. Titanium:Sapphire has a matrix of crystalline aluminium oxide $\left(\mathrm{Al}_{2} \mathrm{O}_{3}\right)$, of the corundum family, where some of the $\mathrm{Al}^{3+}$ ions are substituted by $\mathrm{Ti}^{3+}$ ions.

The crystalline structure of the sapphire crystal is hexagonal trigonal. The symmetry group of this lattice induces a different optical behaviour along on of the axis of the crystal, known as the c-axis. Therefore, the crystal is birefringent uniaxial where the ordinary rays are polarized perpendicular to the c-axis $(\sigma$ polarization) and the extraordinary rays parallel to the axis ( $\pi$ polarization). There are two prominent methods for growing Ti:Sa crystals: Czochralski and HEM. Czochralski is the most extended method for growing sapphire, was explained in section 3.1.1 for Nd:YAG. In HEM (Heat Exchanger Method), or sometimes called inverse Kyropoulos, a seed crystal is mounted on a helium-cooled heat exchanger, which prevents the seed from melting while the furnace fuses the doped sapphire around it. After partial melting of the seed, helium flow is increased in the heat exchanger in order to cool the seed and initiate crystallization in three dimensions while keeping the furnace at constant temperature. This method enables the growth of large crystals and good quality crystals. 


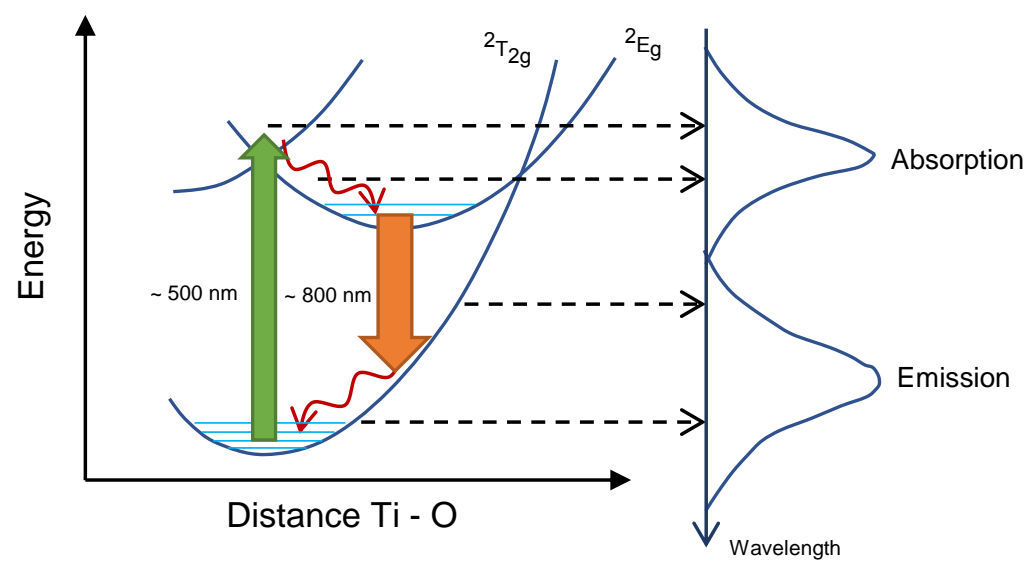

Figure 5.2: Band diagram of Ti:Sa and its correspondence to the absorption and emission cross section. The abscissa represents the displacement of the $\mathrm{Ti}$ ion inside the crystal lattice.

The electronic structure of the $\mathrm{Ti}^{3+}$ ions presents the closed shells of $\mathrm{Ne}$ and a single electron in a $3 \mathrm{~d}$ orbital. This orbital has a five-fold degenerate energy level, ${ }^{2} \mathrm{D}$ in atomic notation, that will be broken by the crystal field. The symmetry group of the lattice can be expressed as a superposition of the cubic and trigonal group. The cubic symmetry breaks the degeneracy of ${ }^{2} \mathrm{D}$ into two levels: ${ }^{2} \mathrm{~T}_{2 \mathrm{~g}}$, the fundamental level, is three-fold degenerate and ${ }^{2} \mathrm{E}_{\mathrm{g}}$, the excited level, two-fold degenerate. The trigonal field splits the fundamental level into two levels ${ }^{2} \mathrm{~A}_{1}$ and ${ }^{2} \mathrm{E}$, which is further split by spin-orbit coupling. Meanwhile, the excited state is split in two by the Jahn-Teller effect.

The simplicity of the energy levels of the $3 \mathrm{~d}$ orbital and the existence of a single excited state diminishes the possibility of upconversion, quenching and other problems with absorption that could decrease its performance as a laser medium.

The emission spectrum of the transition ${ }^{2} \mathrm{E}_{\mathrm{g}}-{ }^{2} \mathrm{~T}_{2 \mathrm{~g}}$ extends from 600 to 1100 $\mathrm{nm}$, as seen in figure 5.1. This is the reason why Ti:Sa is so important for the generation of ultrashort pulses, as this bandwidth can hold pulses as low as 3.6 fs, an optical cycle at $800 \mathrm{~nm}$. The minimum pulse duration achieved with a Ti:Sa laser (including post-compression) is $5 \mathrm{fs}$ [55].

The absorption spectrum is also very large, ranging from 450 to $650 \mathrm{~nm}$, as shown in figure 5.1. It corresponds to the superposition of two absorption bands, 
one centred around $556 \mathrm{~nm}$ and the other on $488 \mathrm{~nm}$, which can be explained by the Jahn-Teller splitting of the excited state. However, the growth process of Ti:Sa may add some amount of unwanted $\mathrm{Ti}^{4+}$ ions to the crystal lattice. The presence of $\mathrm{Ti}^{4+}$ impurities or interstitial $\mathrm{Ti}^{3+}$ ions is the cause of residual absorption around $800 \mathrm{~nm}$.

Due to its birefringent nature, the absorption and emission cross sections depend on the polarization of the electric field. The cross section of the $\pi$ polarization is almost three times larger than the $\sigma$ polarization, so the correct operation of a Ti:Sa laser requires polarization control of both pump and seed beams.

The large absorption and emission bandwidth of Ti:Sa has its origin in the vibronic nature of its molecular configuration. It can be better explained using the $\mathrm{Ti}^{3+}$ ion configuration space (see figure 5.2). The paraboloids represent the energy of the electronic level as a function of the position of the $\mathrm{Ti}^{3+}$ ion while the horizontal bands are the accessible vibronic states.

When the $\mathrm{Ti}^{3+}$ ion is in its fundamental state, it can be found on the lowest energy position in the $\mathrm{T}$ band. When a photon excites the ion, it jumps to the $\mathrm{E}$ band, but notice how it can achieve many vibronic states, which explains the large absorption bandwidth of the Ti:Sa.

Once in the excited state, the ion quickly liberates energy in the form of phonons until it reaches the lowest energy in the excited band. The desexcitation is done through the emission of a photon. Again, the ion de-excites into one of the many possible vibration states in the $\mathrm{T}$ band, which explains the large emission bandwidth, and then liberates photons to reach the fundamental state.

The ion-phonon coupling in the Ti:Sa lattice is not only responsible for the large absorption and emission bandwidths, but it also separates the spectrum so that they only barely overlap.

The excited state has a fluorescence lifetime of $3.3 \mu \mathrm{s}$, which puts a limit on the energy that can be stored. This is a consequence of the large emission cross section of Ti:Sa, as the fluorescence lifetime $\tau_{f}$ is related by [56]

$$
\frac{1}{\tau_{f}}=\frac{8 \pi n^{2}}{c^{2}} \int \nu^{2} \sigma_{e m}(\nu) d \nu
$$

The large absorption bandwidth of Ti:Sa enables several pumping sources that 


\begin{tabular}{ll} 
Ti:Sa & \\
\hline Wavelength $(\mathrm{nm})$ & 800 \\
Lifetime $(\mu \mathrm{s})$ & 3.3 \\
Refractive index & 1.76 \\
Thermal conductivity $(\mathrm{W} / \mathrm{mK})$ & 33 \\
$\mathrm{dn} / \mathrm{dt}\left(10^{-6} K^{-1}\right)$ & 1.2 \\
\hline
\end{tabular}

Table 5.1: Summary of the most relevant optical and thermal parameters of Ti:Sa

have a wavelength that falls under the curve. Historically, Ti:Sa lasers were pumped with CW Argon lasers, which have emission lines in the vicinity of $514 \mathrm{~nm}$ [4]. However, technology evolved towards the use of high power pulsed sources. These needs meant a shift to the use of flash-lamps [57] or colorant lasers [58]. Nowadays, the most extended way of pumping a Ti:Sa laser, both pulsed or CW, is through the second harmonic line of Nd:YAG (532 nm). However all these pump sources are very expensive and inefficient and new research is being performed towards the use of diodes, but recent investigations reported undesired losses at the laser wavelength that are not present on longer pump wavelengths $[59,60]$.

Table 5.1 summarizes the most notable optical and thermal properties of Ti:Sa.

\subsection{CPA laser}

As mentioned in the introduction, the demonstration of chirped-pulse amplification for optical pulses [6] was the breakthrough that allowed to overcome the intensity limitation by conventional means of amplification.

In this work, we have made use of the technique known as chirped pulse amplification CPA in order to amplify the Ti:Sa laser generated pulses. It is similar to a MOPA configuration in that it involves a pulse generator and a chain of amplifiers but includes a pulse stretcher and a compressor in order to lower peak power and avoid damage to the materials involved.

Stretching the pulse involves chirping. Chirping involves using a dispersive element, such as a prism, a grating or more recently grisms, a hybrid element that 


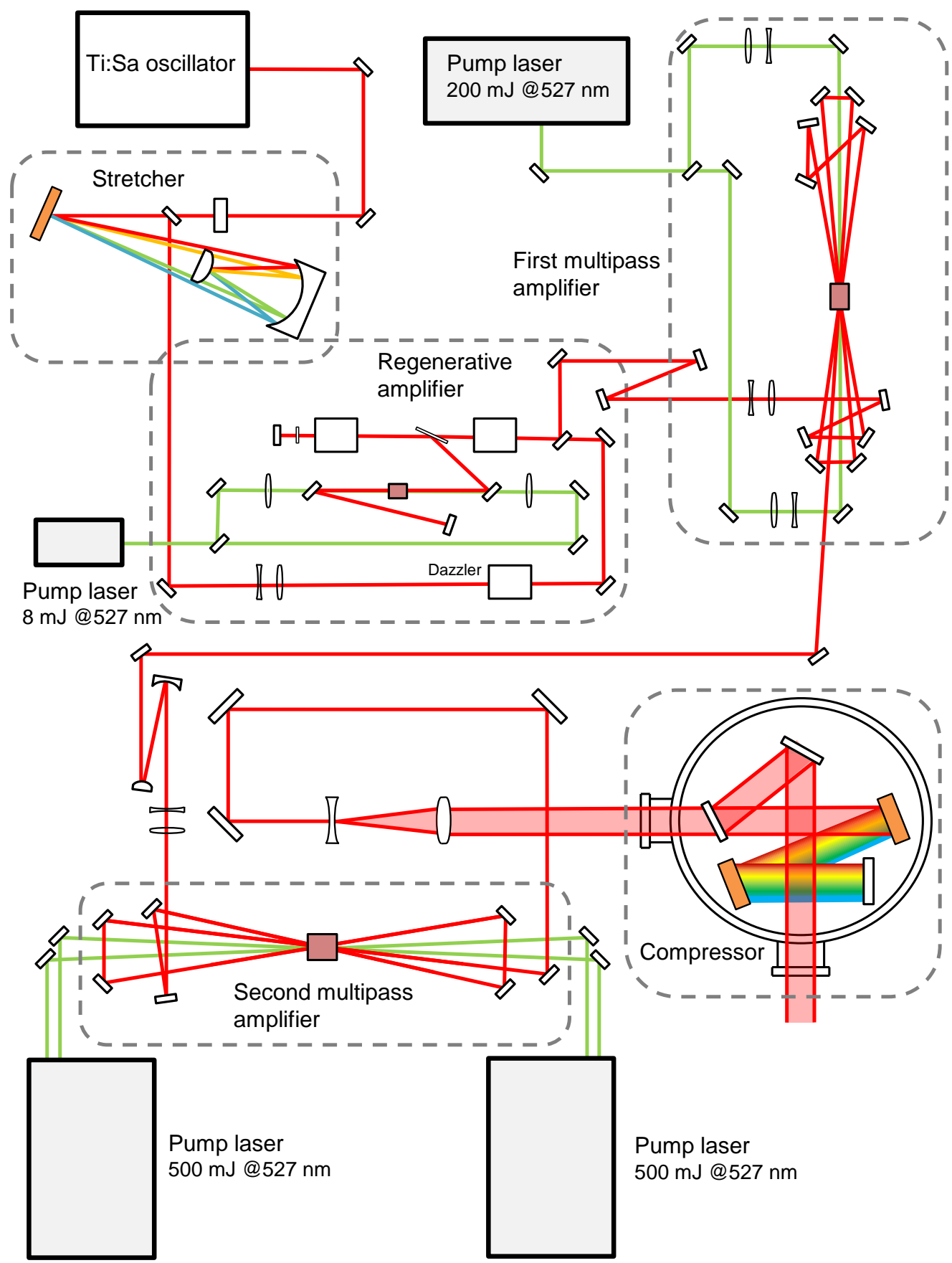

Figure 5.3: Scheme of the CPA Ti:Sa laser developed in the thesis. A pulse oscillator sends a beam of a few femtoseconds that is stretched in time in a pulse stretcher. Then it enters a chain of amplification that starts with a regenerative amplifier that is followed by a first multipass amplifier and ends with a second multipass amplifier. The CPA chain ends with a compressor installed in a vacuum chamber. 


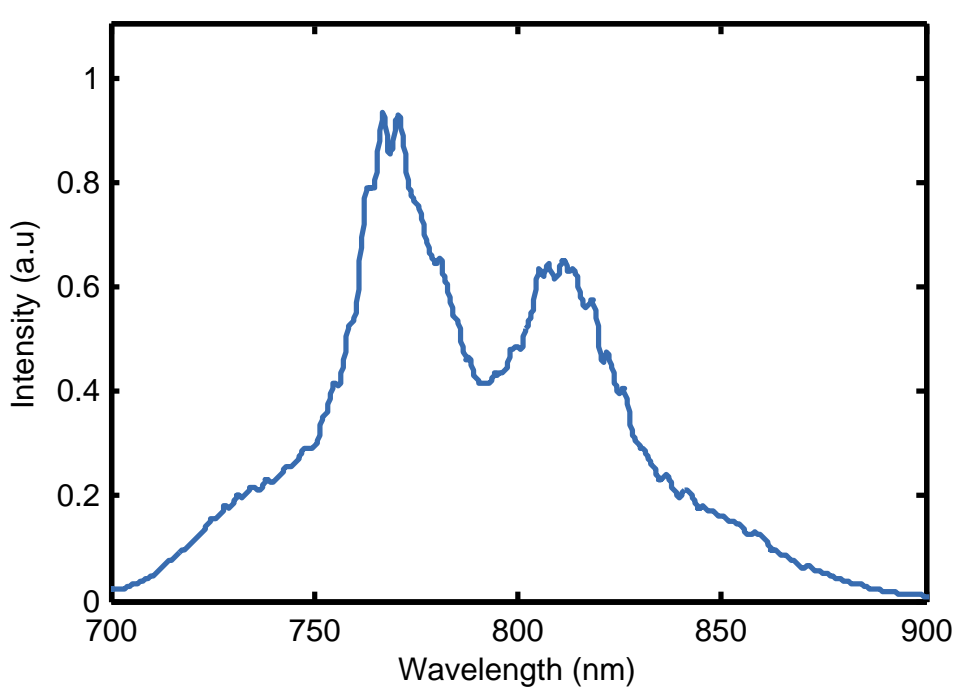

Figure 5.4: Spectrum captured at the output of the oscillator.

combines a prism and a diffraction grating. The sign of the chirping is important too: for a positive chirping the two gratings must have a negative distance between them and this is much easier to achieve in a stretcher, where the pulse energy is low; therefore, most systems work stretching the pulse with negative chirping and recompressing it with a positive chirp compressor. In order to recompress the pulse back to its initial duration, several conditions must be met: the stretcher and compressor must be conjugates of each other, the pulse must retain its full bandwidth and the amplification must be linear. However, these conditions are hard to meet in a real setup and the pulse that exits the compressor is generally longer than the input pulse.

The scheme of the CPA laser developed in this thesis is presented in fig. 5.3. A femtosecond oscillator generates a pulse with a very broad bandwidth and very low energy and an Öffner stretcher enlarges the pulse up to 10000 times. The amplification process starts with a regenerative amplifier which increases the energy per pulse as far as $1.5 \mathrm{~mJ}$. Then, two multipass amplification stages set the energy of the laser to $315 \mathrm{~mJ}$. Finally, a compressor shortens the pulse to 45 fs and energy of $265 \mathrm{~mJ}$. 


\subsubsection{Femtosecond oscillator}

The laser amplification chain starts with a Kerr-lens mode locked Ti:Sa laser oscillator. It is based on a commercial design manufactured by Venteon ${ }^{1}$. The Brewster-cut Ti:Sa crystal is pumped by a CW Nd:YAG laser with a total output power of $8 \mathrm{~W}$ in $532 \mathrm{~nm}$ wavelength is injected into the resonator via fibre. The output of this oscillator is a train of $5 \mathrm{~nJ}$ pulses at $80 \mathrm{MHz}$, resulting in $0.4 \mathrm{~W}$ average power.

The spectrum is ultra-broadband as a result of self-phase modulation in the Ti:Sa crystal. It spans from $700 \mathrm{~nm}$ to $900 \mathrm{~nm}$ and has a small cleft in the vicinity of $800 \mathrm{~nm}$, as seen in figure 5.4, which is capable of supporting pulses as short as 12 fs according to the manufacturer. This feature probably results from an unoptimized output coupler reflectivity.

\subsubsection{Stretcher}

The purpose of the stretcher in a CPA system is to stretch in time the pulse coming from the oscillator. The pulse duration can be manipulated by changing the spectral phase which is achieved typically with diffraction gratings or prisms. The most important term is second order group delay dispersion (GDD). Superior orders do not contribute critically to the pulse duration but contribute significantly to the temporal structure so they must be controlled too.

The second order spectral phase introduced in a grating stretcher can be calculated from [61]:

$$
\frac{d^{2} \phi(\omega)}{d \omega^{2}}=\frac{\lambda^{2} L_{g}}{\pi c^{2} d^{2}}\left(1-\left(\frac{\lambda}{d}-\sin \gamma\right)^{2}\right)^{-3 / 2}
$$

Which depends on the incidence angle of the laser, the line density of the grating and the distance between gratings. The sign of this magnitude shapes the spectral distribution: a positive sign means that the shorter wavelengths will travel faster than the longer wavelengths and vice versa for the negative sign. This linear redistribution of frequencies inside the pulse, known as chirping, has the effect of stretching the pulse in time. The stretcher is the device that introduces GDD in

\footnotetext{
${ }^{1}$ Venteon, Germany venteon.com
} 


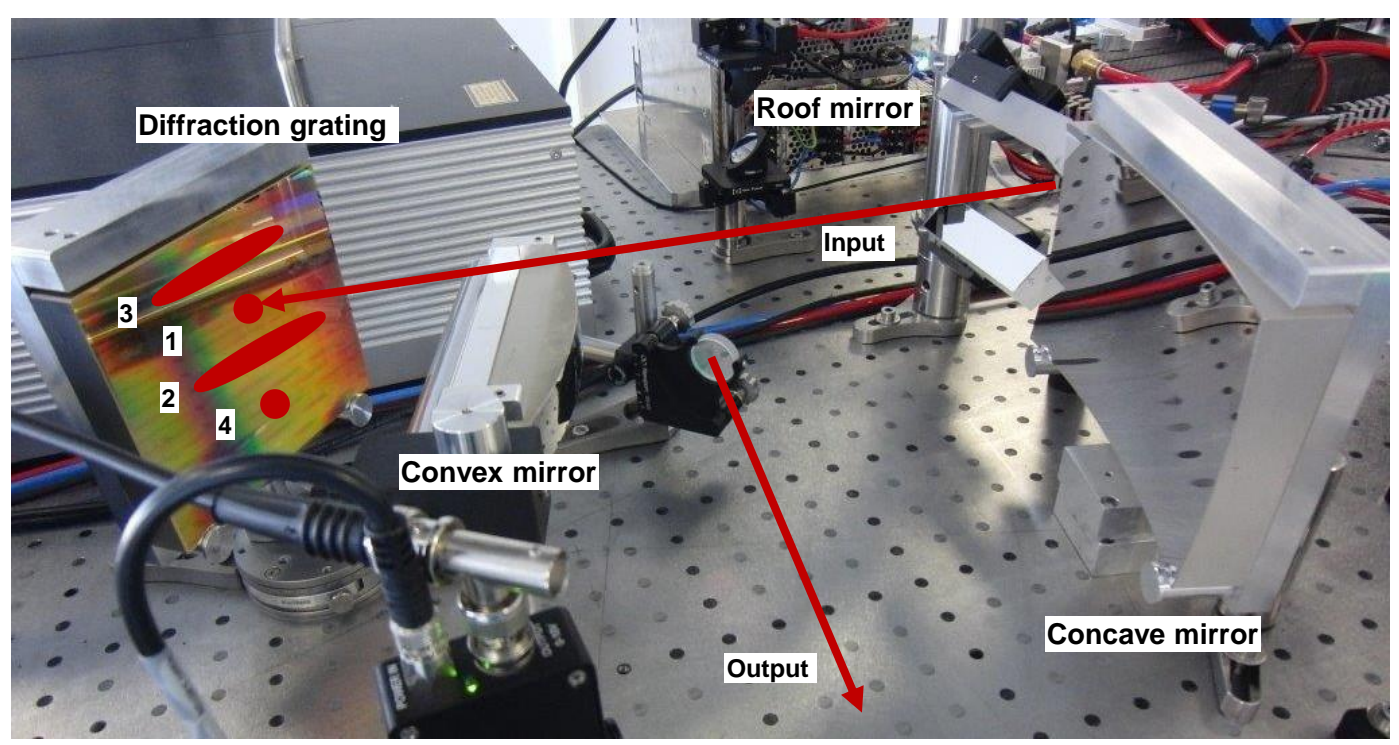

Figure 5.5: Image of the pulse stretcher, consisting of a convex and a concave mirror, a diffraction grating and a roof mirror. The input pulse impinges the grating (1) and is diffracted towards the concave-convex mirror arrangement which reflects it back to the grating (2). A roof mirror changes the height of the beam (3) which goes back through the mirrors until the pulse is stretched in time (4).

the pulse; for an optimum stretching/compression, the compressor must introduce an equal amount of GDD but of the opposite sign.

The design of the stretcher must take into account the amplified bandwidth and the desired pulse duration necessary for a damage-free operation. Both are linked by the stretcher/compressor dispersion and are governed by the requirements of the amplifier design. The amplified bandwidth is limited by the bandwidth of the amplifier medium and is further limited by gain narrowing.

The configuration of the stretcher assembled as a part of this thesis is a singlepass Öffner type stretcher, which was chosen due to its simplicity and its aberrationfree design [62]. It is comprised of one gold-coated reflecting diffraction grating (Horiba Jovin-Yvon) $^{2}$ with a line density of 1200 lines/mm and $92 \%$ reflectivity at $800 \mathrm{~nm}$ according to the manufacturer, tilted an angle of $60^{\circ}$ and a relay system of two spherical mirrors with a radius of curvature of $500 \mathrm{~mm}$ and $-250 \mathrm{~mm}$ respectively. These two mirrors create a virtual image of the grating $20 \mathrm{~cm}$ away from the real one, creating a path of effective negative distance introducing positive dispersion in the pulse. The grating is impinged four times at different heights by the input

\footnotetext{
${ }^{2}$ Horiba Jovin-Yvon, France horiba.com
} 
pulse thanks to a roof mirror, as pictured in figure 5.5. Thanks to this design, the spatial characteristics of the input beam should remain unaffected, if the setup is aligned correctly. The pulse duration of a stretched pulse after traversing the stretcher was estimated to be more than 150 ps using a fast photodiode.

Higher order dispersion terms will be added to the pulse during the propagation of the pulse through the following amplification stages, primarily third order dispersion (TOD). This higher order term generates a deformation of the pulse shape and is specially problematic for a stretcher/compressor based on diffraction gratings because it cannot compensate the third order dispersion introduced by all the materials involved in the amplification chain. Its compensation requires an external device, such as a Dazzler ${ }^{3}$, used in this thesis, which is a programmable acousto-optic device capable of shaping both the spectral intensity and phase of the stretched pulse. This device encloses a $\mathrm{TeO}_{2}$ crystal which is highly birefringent and, with the addition of an acoustic wave, acts similarly to a Bragg grating [63], and as a result various spectral components are diffracted at different positions through the crystal acquiring different group delays, while the spectral amplitude is controlled through the local acoustic intensity. However, the Dazzler has some limitations that are relevant for the performance of the stretcher and even amplification stages since the amount of GDD or TOD it can introduce is limited and strongly affects the efficiency of the device.

\subsubsection{Regenerative amplifier}

The stretched pulse is amplified for the first time in a regenerative amplifier. This scheme is specially intended for the amplification of low energy pulses since it supports the potential for a large number of passes through the active medium. A regenerative amplifier is also suitable in the situation where the laser crystal has low gain per pass.

The operation principle of regenerative amplification is the injection of a pulse into a resonant cavity that includes a gain medium, and as the pulse is trapped into the cavity it carries out a large number of passes while its energy builds up and the gain decreases. When the losses in the resonator equal the gain of the

\footnotetext{
${ }^{3}$ Fastlite, France www.fastlite.com
} 


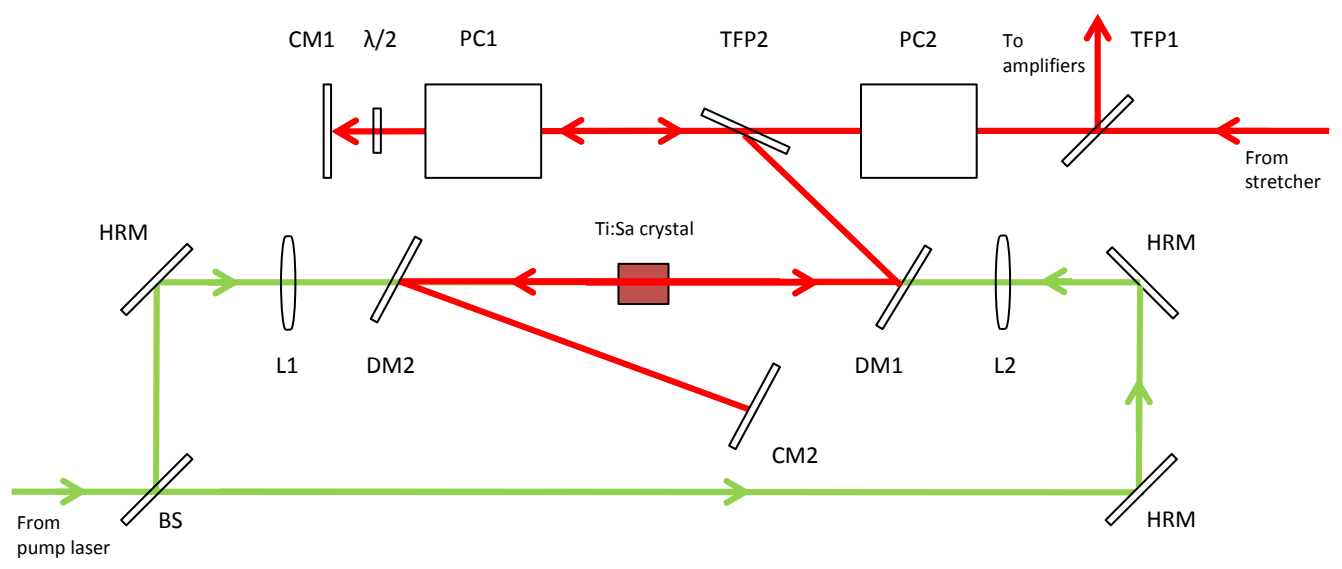

Figure 5.6: Scheme of the regenerative amplifier. BS is a polarizer beam splitter. HRM are the high reflective mirrors for the pump wavelength. CM1 and 2 are the cavity curved mirrors. DM1 and 2 are dichroic mirrors, reflective for the seed but transmissive for the pump wavelength. L1 and L2 are convex lenses that focus the beam into the Ti:Sa crystal. TFP1 and TFP2 are two thin film polarizers. PC1 and $\mathrm{PC} 2$ are the Pockels cells and $\lambda / 4$ is a quarter waveplate.

amplifier a maximum energy is reached and it is the ideal time to couple the pulse out of the cavity. The in/out coupling of the pulses is accomplished by controlling the polarization throughout its path using a Pockels cell and a polarizer.

The ability to make a lot of passes make these kind of amplifiers have a high cumulative gain while the cavity suppresses high order modes and improves the spatial quality of the beam. The resonator also provides stability to the laser energy.

The setup of the regenerative amplifier is pictured in 5.6. The active medium is a Ti:Sa crystal with rectangular end faces and a size of $4 \times 6 \mathrm{~mm}$ and $20 \mathrm{~mm}$ long. The faces of the crystal are anti-reflection coated but in order to preserve the polarization of the laser throughout all the passes, two fused silica Brewster-angled windows are placed at both entrance and exit of the crystal.

The pump laser is of the regenerative amplifier is an Empower 15 (SpectraPhysics) ${ }^{4}$ which delivers $8 \mathrm{~mJ}$ and pulses of $200 \mathrm{~ns}$ at $100 \mathrm{~Hz}$. Using a beam splitter, the beam is divided in two in order to pump the Ti:Sa crystal from both sides with half the energy, which provides a more uniform distribution of energy and heat

\footnotetext{
${ }^{4}$ Spectra-Physics, USA, spectra-physics.com
} 


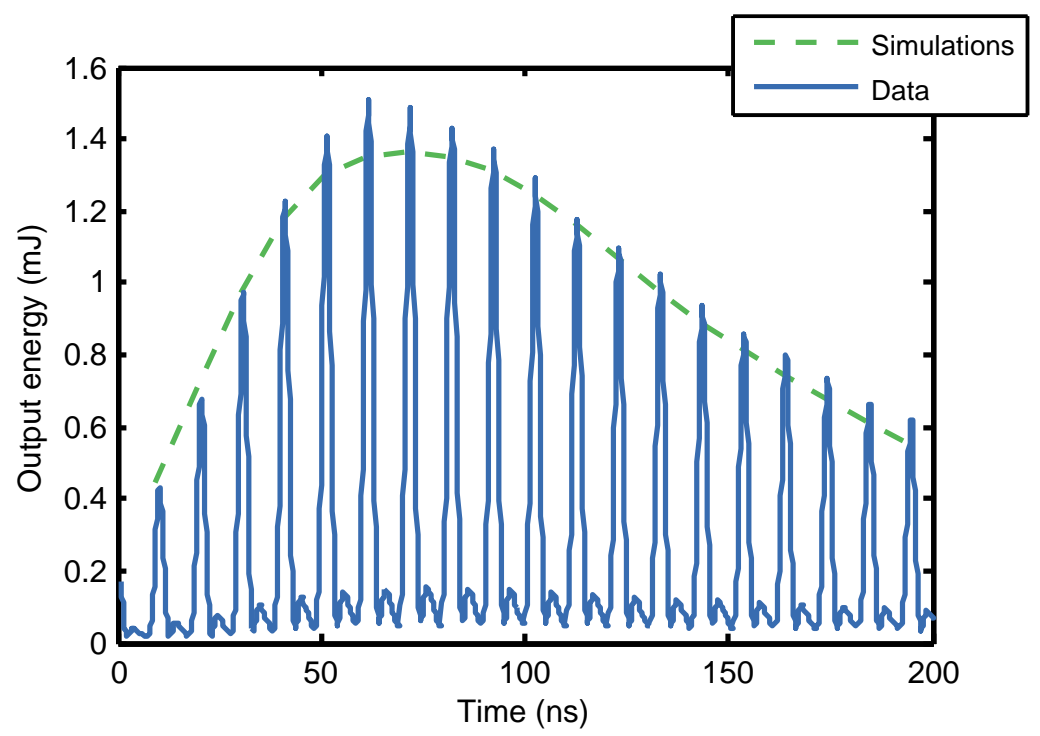

Figure 5.7: Performance of the regenerative amplifier. Dashed line represents the simulations.

inside the active medium in the longitudinal direction.

The amplification model developed in section 2.1 can be adapted to the case of a regenerative amplifier. In the case of multiple passes through the gain medium, the Frantz Nodvik equation 2.14 can be written as:

$$
F_{\text {out }}^{i}=F_{\text {sat }} \ln \left(1+\exp \left(\frac{F_{\text {sto }}^{i}}{F_{\text {sat }}}\right)\left(\exp \left(\frac{F_{\text {in }}^{i}}{F_{\text {sat }}}-1\right)\right)\right)
$$

The input, output and stored fluence are dependent on the previous pass. Assuming that there are no losses, the stored energy in each pass $i$ can be calculated as the difference between its value in the $i-1$ pass and the extracted energy:

$$
F_{\text {stor }}^{i+1}=\left(F_{\text {stor }}^{i}-\left(F_{\text {out }}^{i}-F_{\text {in }}^{i}\right)\right) \exp \left(T / \tau_{f}\right)
$$

The factor $\exp \left(T / \tau_{f}\right)$, where $T$ is the time between two consecutive passes through the active medium and $\tau_{f}$ is the fluorescence lifetime, accounts for the losses by spontaneous emission. For this regenerative amplifier, the time that it takes a pulse to travel through the Ti:Sa is $L / 2 c$ which is about $9 \mathrm{~ns}$, which is much less that the fluorescence lifetime of $3.3 \mu \mathrm{s}$ of Ti:Sa and this factor can be ignored.

While in this model it is considered that there are no absorption losses in the crystal, a real amplifier can have several sources of losses, such as leaks in the 


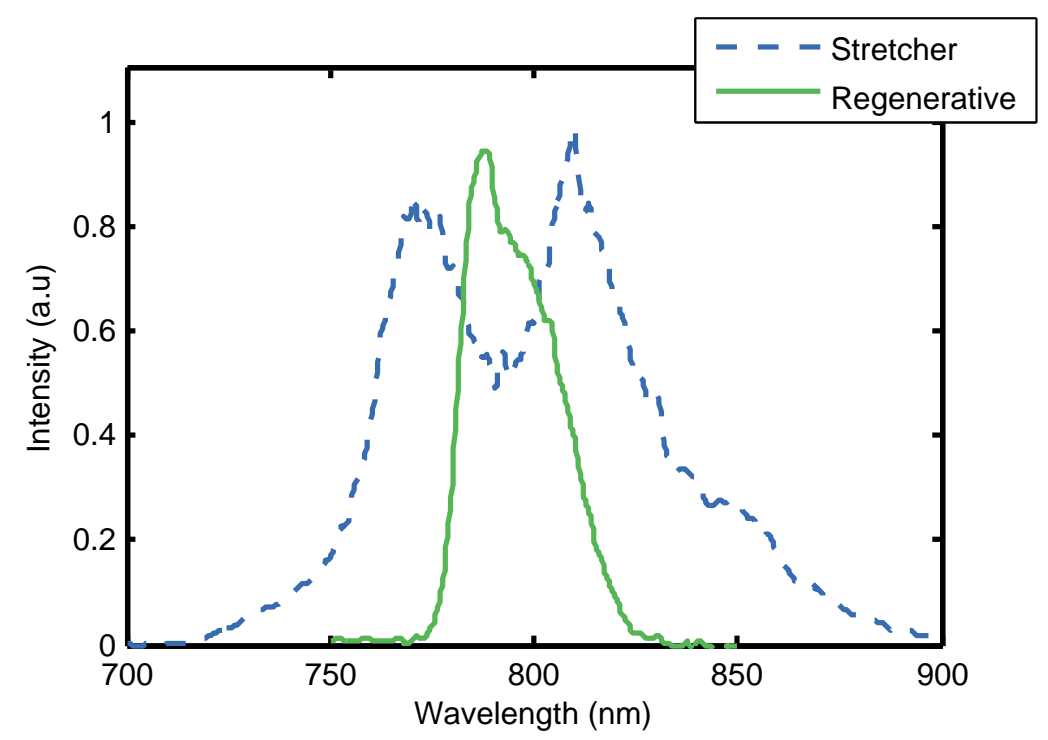

Figure 5.8: Spectrum of the output of the regenerative amplifier (green) versus the output spectrum of the stretcher (blue dashed line).

mirrors and in the thin-film polarizer, scattering in the Pockels cell, etc. These contributions can be introduced as a loss in each pass, $\delta$, as:

$$
F_{\text {in }}^{i+1}=(1-\delta) F_{\text {out }}^{i}
$$

In this case $\delta=16 \%$; the thin-film polarizer contributed to the majority of the losses in the regenerative amplifier.

The result of amplification in the regenerative amplifier is an output beam with an energy of $1.5 \mathrm{~mJ}$ per pulse and a beam diameter of $1.2 \mathrm{~mm}$. The beam profile is gaussian shaped, which is one of the most important advantages of a regenerative amplifier since the resonator naturally filters the spatial modes. The resonator measured a total of $1.5 \mathrm{~m}$ between the two cavity mirrors, CM1 and CM2 in figure 5.6 .

The spectrum of the pulse that exits the regenerative amplifier is pictured in figure 5.8. The output spectrum is noticeably narrower than the input. It is limited by many factors such as the finite bandwidth of the thin-film polarizer that couples/uncouples the pulses from the regenerative amplifier and gain narrowing.

Preceding the next amplifier a Faraday isolator is included in order to prevent feedback from the following amplifiers. This tool is critical since in its absence, backreflected light from the next amplification stage entered the resonator, gen- 


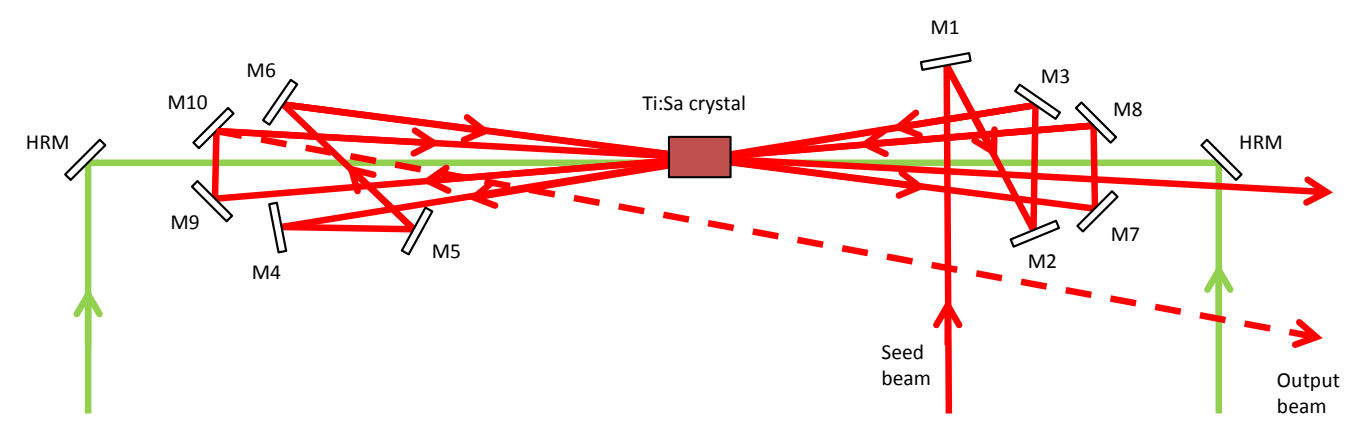

Figure 5.9: Scheme of the first multipass amplifier with tree passes. HRM are high reflector mirrors for the pump beam wavelength and M1-10 represent are the mirrors of the multipass. M4 is a convex curved mirror

erating distortions in the regenerative performance, such as modifying the beam profile of the output pulse and creating a QCW component that could be spotted by a photodiode. If unchecked, the performance of the rest of amplification stages dropped significantly since this long pulse component depleted the gain of the amplifiers before the main pulse arrived.

One of the drawbacks of a regenerative amplifier is the generation of artefacts in the temporal profile of the laser, in particular replicas of the main pulse. These replicas are created by the leaks of the thin-film polarizer that couples out the pulse during the build-up of amplification and therefore are separated in time by a roundtrip of the resonator. This effect can be detrimental for the rest of amplification stages and will be explored in detail later in this chapter.

\subsubsection{First multipass amplifier}

After the regenerative amplifier, the next stage in the amplification chain is a multipass amplifier. It is pumped by up to $200 \mathrm{~mJ}$ in $15 \mathrm{~ns}$ pulses at $100 \mathrm{~Hz}$ from a Nd:YLF laser. This nanosecond laser is a was not presented in detail because it is a smaller version of the MOPA lasers discussed in chapter 4. It includes two PH5400 pumping heads loaded with $5 \mathrm{~mm}$ diameter Nd:YLF rods, one working as an oscillator and one as an amplifier. A KTP crystal converted to second harmonic with an efficiency of $55 \%$. The output energy at $527 \mathrm{~nm}$ can be adjusted up to 200 mJ per pulse.

The Ti:Sa crystal is plane-parallel, cylindrical, $6 \mathrm{~mm}$ in diameter and $15 \mathrm{~mm}$ of 


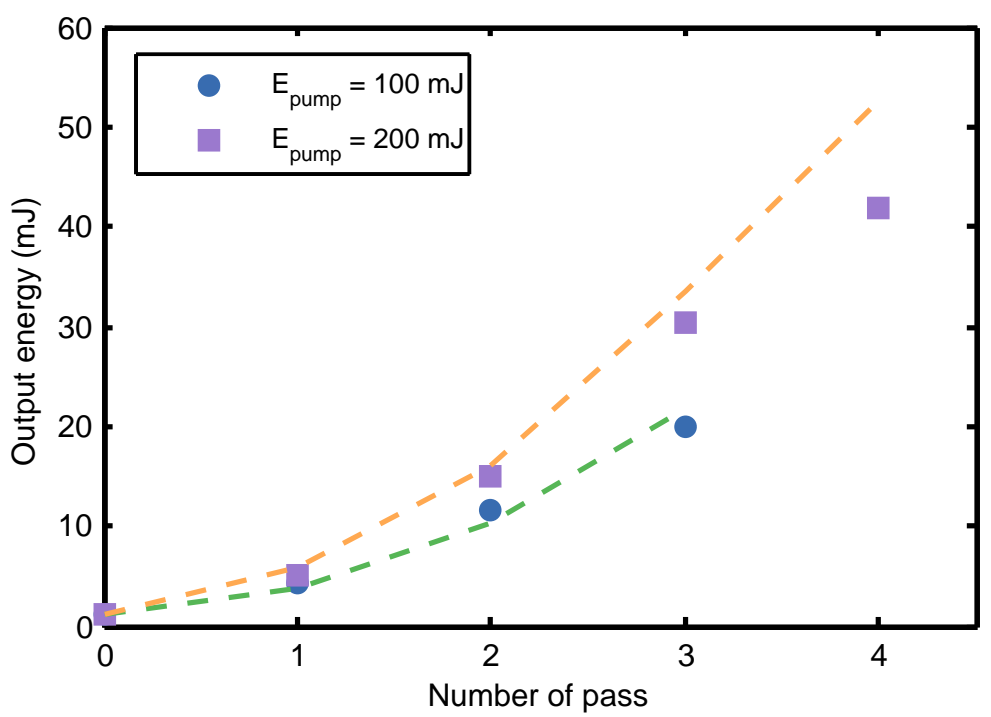

Figure 5.10: Performance of the first multipass amplifier for total pumped energy of $100 \mathrm{~mJ}$ (circles) and $200 \mathrm{~mJ}$ (squares). Dashed line represents the simulations using Frantz-Nodvik.

length, absorbs $92 \%$ of pump light and is placed on a copper water-cooled mount. This amplifier is constructed in a multipass bow tie configuration, presented in figure 5.9, where the seed beam undergoes three or four passes through the gain medium just by changing the orientation of one of the mirrors, M10 in figure 5.9, for three passes and four passes. The laser beam enters collimated from a telescope situated after the Faraday isolator and is directed towards the Ti:Sa crystal by a set of plane mirrors up until the second pass where a convex mirror, M4, is used to introduce divergence to the beam in order to counter the action of thermal lensing. This mirror is kept at an incident angle of less than $10^{\circ}$ to prevent the addition of spherical aberration.

The maximum output energy per pulse achieved in this amplifier is $41 \mathrm{~mJ}$ in four passes with $200 \mathrm{~mJ}$ of pump energy and an input pulse energy of $1.4 \mathrm{~mJ}$. The evolution of the energy in each pass can be observed in figure 5.10. It can be remarked that the gain per pass is about 2.5 for $200 \mathrm{~mJ}$ of pump energy up until the fourth pass, where it is reduced to 1.3; this effect is due to depletion of stored energy in the central part of the crystal. The Frantz-Nodvik calculations were found to be in good agreement with the experimental data.

Thermal lensing begins to be problematic at this stage, since the active medium 


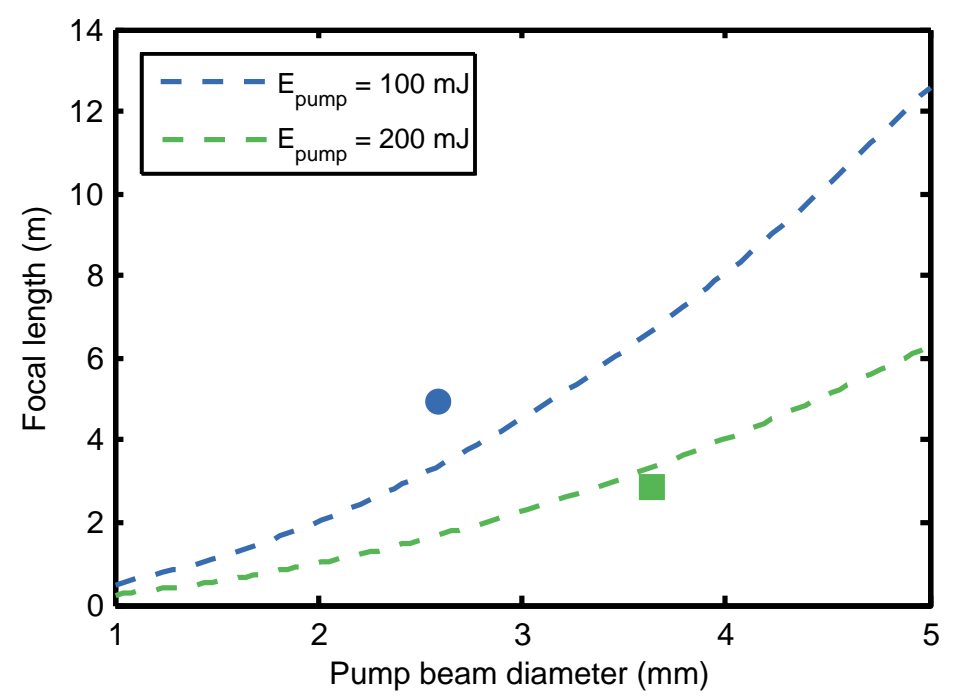

Figure 5.11: Focal length of the thermal lens for pump energy of $100 \mathrm{~mJ}$ and $200 \mathrm{~mJ}$. Straight line represents the simulations and the points are the measured thermal lens.

is pumped by a maximum of $20 \mathrm{~W}$ of optical power, of which about $8 \mathrm{~W}$ are converted to heat. In order to measure the focal length of the Ti:Sa crystal a He-Ne laser is directed at a small angle to the pumped rod and its size measured at different positions with a CCD camera. The results are pictured in figure 5.11 and supported by equation 3.12 for pump powers of 10 and $20 \mathrm{~W}$.

The focal length of this crystal was found to be $5 \mathrm{~m}$ when pumped with a fluence of $2 \mathrm{~J} / \mathrm{cm}^{2}$ using $100 \mathrm{~mJ}$ pump pulses or $2 \mathrm{~m}$ when using $200 \mathrm{~mJ}$ pulses, in solid accordance with the calculations. This effect, if unchecked, can lead to poor beam quality and damage in any of the optics or the active medium, as the beam makes up to four passes through this crystal. In the three pass configuration, the laser leaves the amplifier with a beam diameter of $2.5 \mathrm{~mm}$, while in the four pass configuration, the laser leaves with a slightly smaller diameter of $2.4 \mathrm{~mm}$ and converging in the case of $100 \mathrm{~mJ}$ pump energy and $3.4 \mathrm{~mm}$ in the case of $200 \mathrm{~mJ}$ pump energy.

The beam coming from the pump laser is divided into two branches of equal energy in order to pump the laser from two directions and generate a more homogeneous longitudinal absorbed power distribution. In both branches a telescope is used to modify the size of the beam to provide the required pump fluence at 


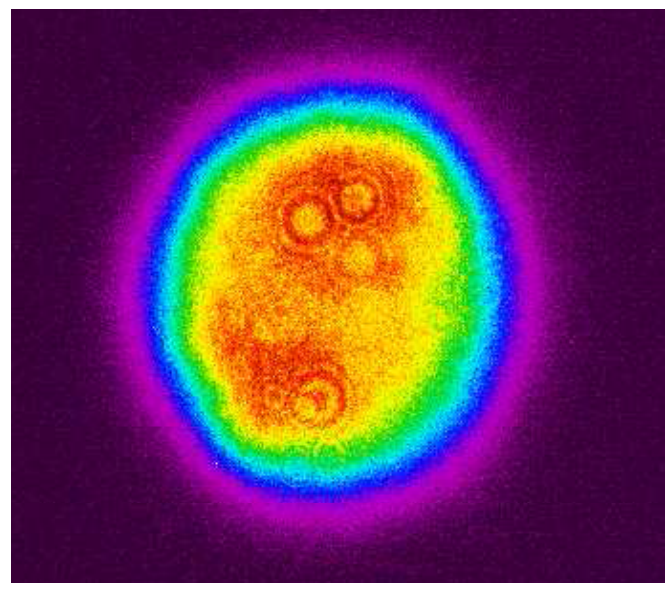

(a)

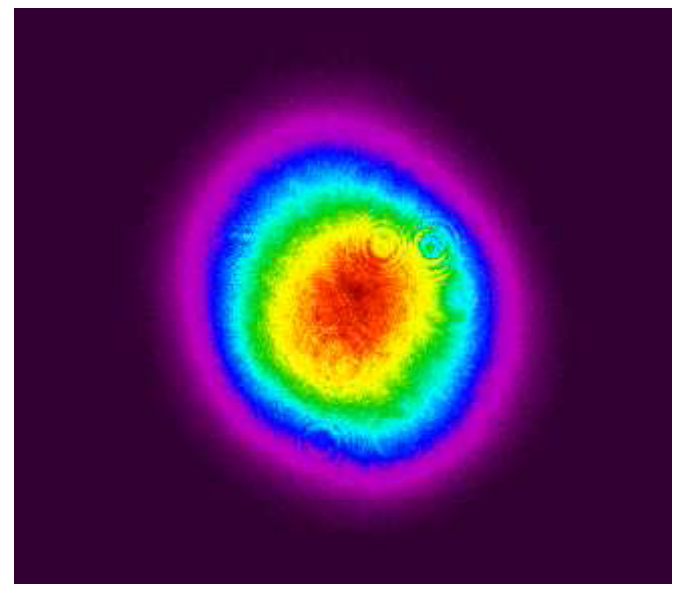

(b)

Figure 5.12: Beam profile of the pump beam at the position of the Ti:Sa crystal. (a) pump energy of $200 \mathrm{~mJ}$ (b) pump energy of $100 \mathrm{~mJ}$.
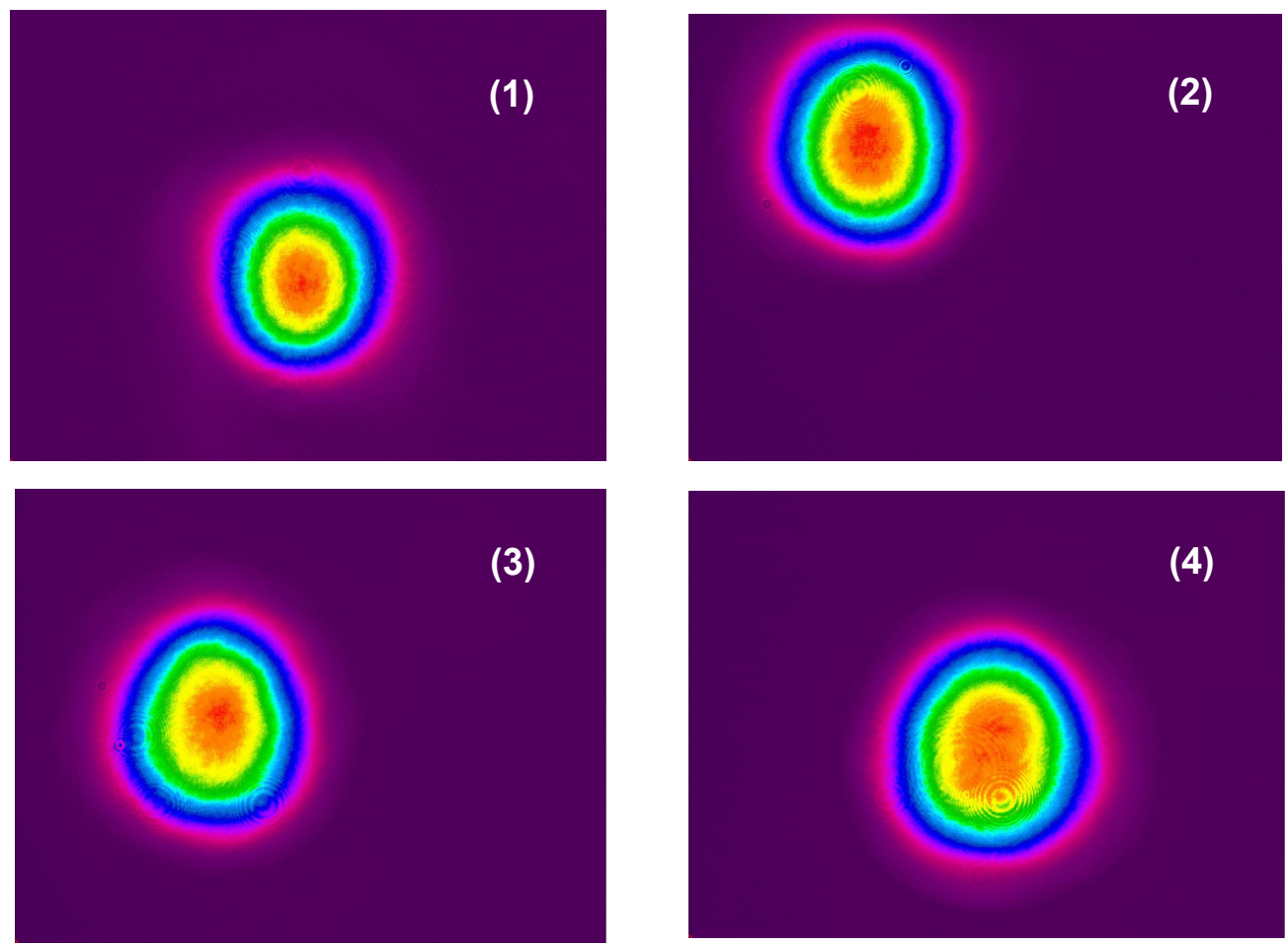

Figure 5.13: Beam profile of the amplified beam for the first (1), second (2), third (3) and fourth (4) passes through the Ti:Sa crystal. 


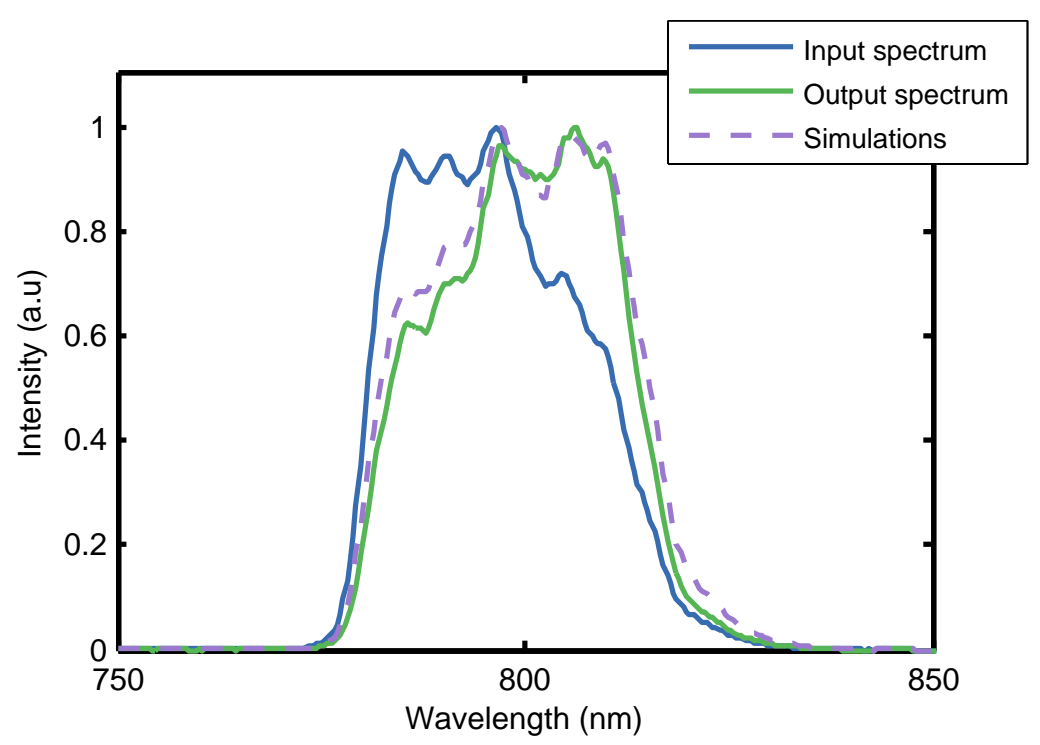

Figure 5.14: Blue: spectrum of the input beam to the first multipass amplifier. Green: output spectrum of the amplified beam. Dashed line: simulations of the spectrum (see chapter 2).

the position of the Ti:Sa crystal. The beam profile of the pump lasers for $100 \mathrm{~mJ}$ and $200 \mathrm{~mJ}$ pump power is pictured in figure 5.12(a) and 5.12(b) respectively. The beam sizes are $3.6 \mathrm{~mm}$ and $2.5 \mathrm{~mm}$ of diameter which correspond to a fluence of 2 $\mathrm{J} / \mathrm{cm}^{2}$.

As for the beam profile of the seed laser, it retains its gaussian shape through amplification, as can be observed in figure 5.13, while the beam size increases to a maximum of $3 \mathrm{~mm}$ from $1.5 \mathrm{~mm}$, keeping the peak fluence close to $1.5 \mathrm{~J} / \mathrm{cm}^{2}$ in order to avoid risk of optical damage.

The spectrum at the output of this amplifier is shown in figure 5.14. The effect of gain narrowing decreases the bandwidth of the pulse from 20 to $18 \mathrm{~nm}$ while gain shifting drifts the spectrum towards the red. The simulations of the spectrum performed using equation 2.30 show good agreement with experimental data.

\subsubsection{Second multipass amplifier}

After the first multipass amplifier there is a second multipass which boosts the energy per pulse towards hundreds of $\mathrm{mJ}$ and is pumped by four times more energy than the previous amplifier, enough to produce TW-level pulses after compression.

The amplifier is built in a multipass bow tie configuration with 3 passes, as 


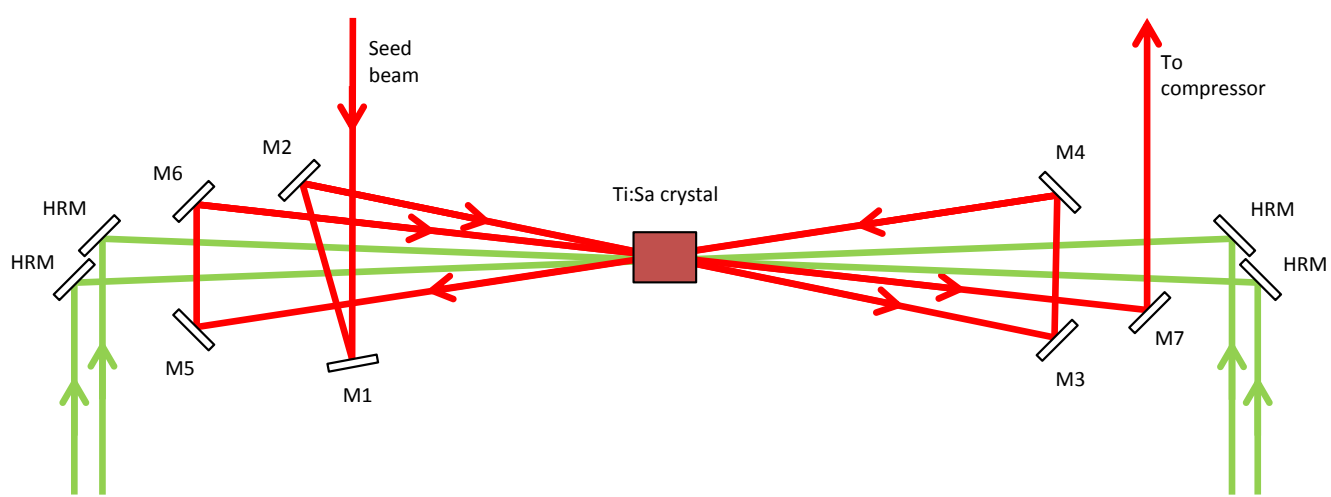

Figure 5.15: Scheme of the second multipass amplifier with three passes. HRM are high reflector mirrors for the pump beam wavelength and M1-10 represent the mirrors of the multipass amplifier.

shown in figure 5.15. One of the mirrors, M5, placed before the laser makes the second pass is a convex mirror of radius of curvature $2000 \mathrm{~mm}$.

The Ti:Sa active medium is a plane-parallel crystal with $16 \times 20 \mathrm{~mm}^{2}$ rectangular faces and $20 \mathrm{~mm}$ length. It was grown using HEM, and purchased from Crystal Systems ${ }^{5}$. It absorbs $93 \%$ of the incoming pump light.

The crystal is cooled in an especially designed copper mount. The cooling liquid flows through a series of microchannels close to the copper contact surface. The mount has two pieces which enable to perfect clamping of the Ti:Sa crystal, which is surrounded by a layer of Indium for maximum surface contact that optimizes heat transfer efficiency between crystal and mount. The mount and the crystal were placed inside a vacuum chamber with a scroll vacuum pump that can reach up to $10^{-2}$ mbar. This is necessary if the cooling temperature is set below $15^{\circ} \mathrm{C}$, as at atmospheric pressure water vapour would condensate on the faces of the crystal. The premise for reducing the temperature of the crystal is that the conductivity of Ti:Sa becomes higher as temperature decreases [64].

The windows of the vacuum chamber parallel to the end faces of the Ti:Sa crystal are anti-reflection coated for both $800 \mathrm{~nm}$ and $532 \mathrm{~nm}$. The crystal is cooled though a dedicated chiller from Julabo ${ }^{6}$, which is able to reach temperatures down to $-20{ }^{\circ} \mathrm{C}$. In order to achieve such a low temperature, the cooling liquid was a

\footnotetext{
${ }^{5}$ GT Crystal Systems Inc, USA, gtat.com

${ }^{6}$ Julabo GmbH, Germany, julabo.com
} 


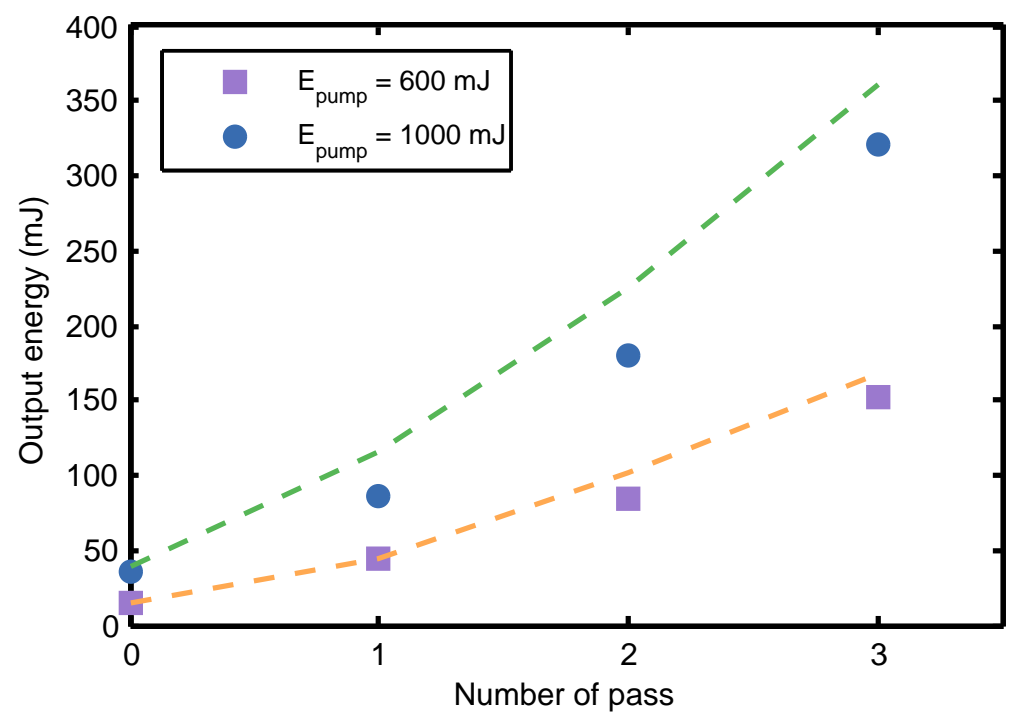

Figure 5.16: Performance of the second multipass amplifier with a pump energy of $600 \mathrm{~mJ}$ (purple squares) and $1000 \mathrm{~mJ}$ (blue circles). The dashed lines represent the simulations.

mixture of water and $20 \%$ ethylene glycol. The addition of this aggregate reduces the freezing temperature of water while keeping its cooling properties reasonably unaltered.

The performance of the amplifier for two different values of the pump energy and a comparison with the Frantz-Nodvik equation is shown in figure 5.16. A maximum energy of $315 \mathrm{~mJ}$ per pulse was reached from an input energy of 36 mJ using a total pump of $1 \mathrm{~J}$. This indicates an amplification efficiency of $31 \%$, matches reasonably well that other multiterawatt Ti:Sa lasers in literature $[65,66]$. The simulations agree reasonably well with the experimental data, in particular when pumped with $600 \mathrm{~mJ}$. The agreement is worse for the $1 \mathrm{~J}$ pump; this may be due to the fact that for large beams it is difficult to measure its precise size, and the input of the Frantz-Nodvik equations becomes less reliable.

The pumping lasers are two identical Nd:YLF MOPA with five pumping heads each, detailed in section 4.3. A total of $1 \mathrm{~J}$ of pumping power divided in four beams, two from each side enter the Ti:Sa crystal. A set of telescopes ensure the beam size is optimal in the position of the crystal, about $6.5 \mathrm{~mm}$ for the maximum pump level, as this means a fluence of $2 \mathrm{~J} / \mathrm{cm}^{2}$; however the telescopes must be designed in such a way that the beam does not converge after travelling through the Ti:Sa 

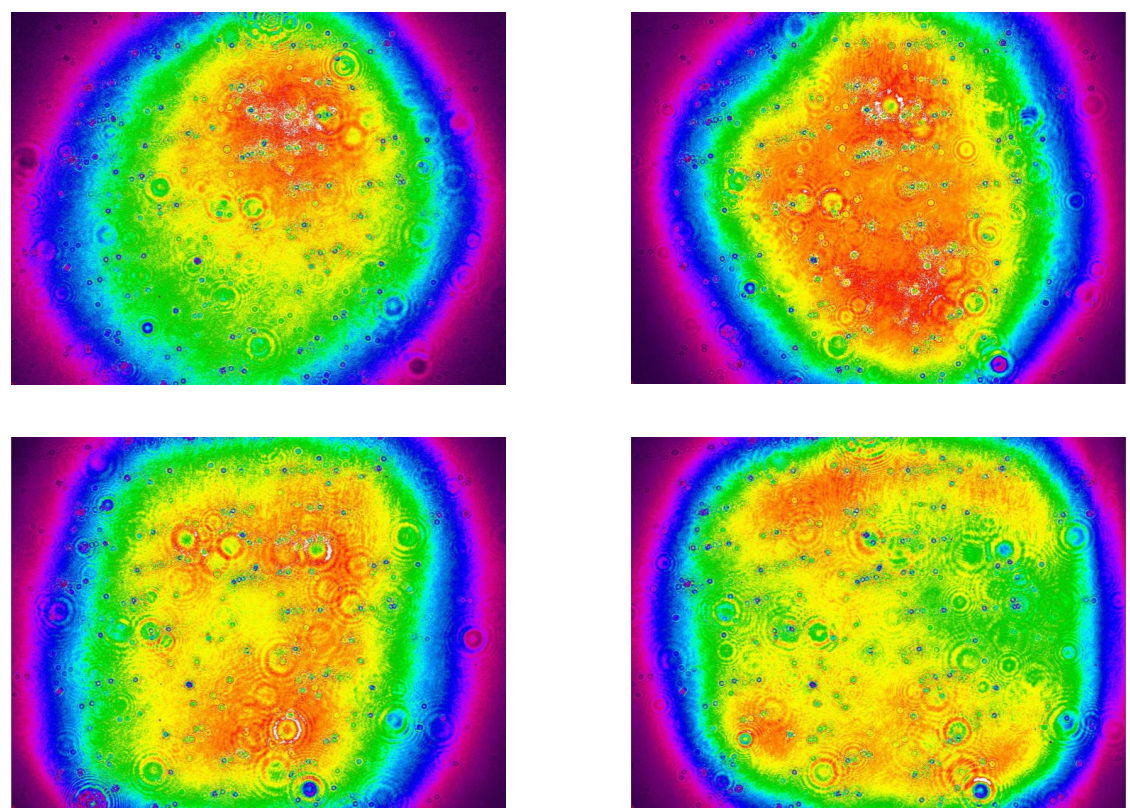

Figure 5.17: Pump profiles at the crystal of the second multipass amplifier from each beam of two pump lasers.
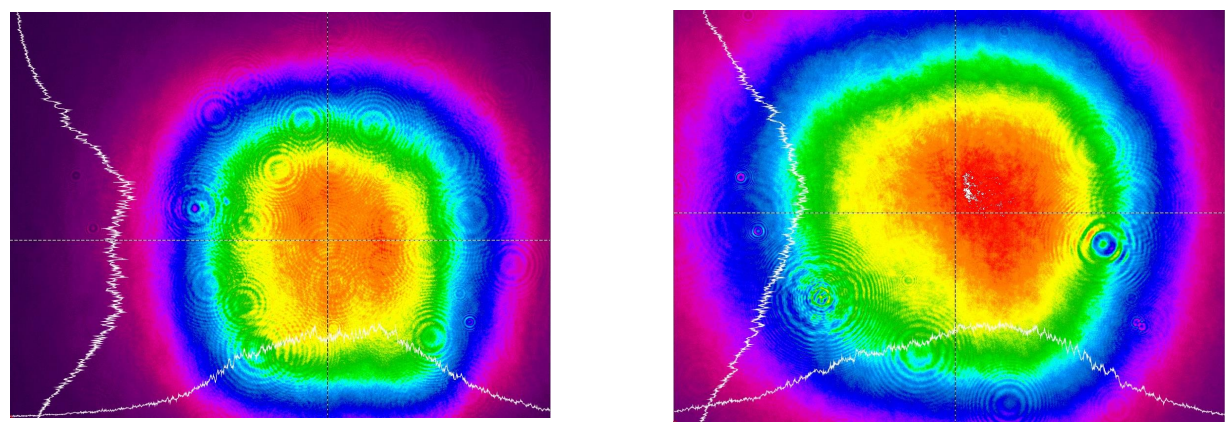

(1)

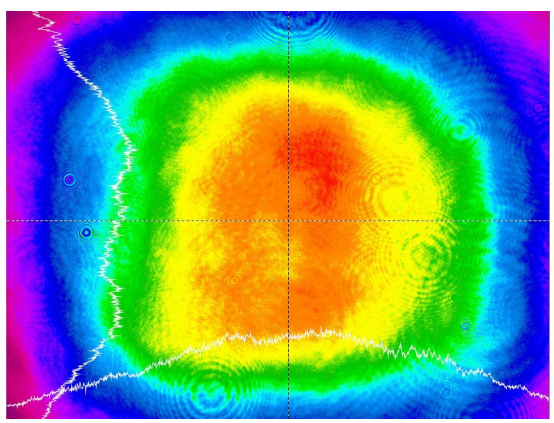

(2)

Figure 5.18: Near-field beam profiles at the crystal of the second multipass amplifier for the first (1), second (2) and third (3) passes through the Ti:Sa crystal. 


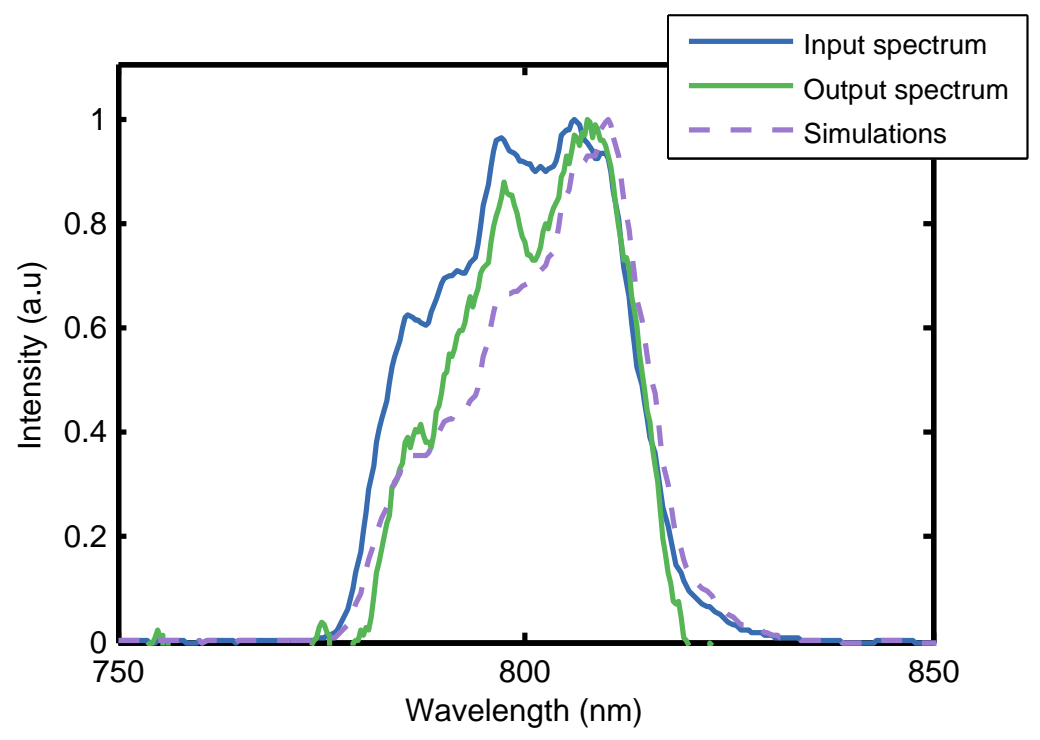

Figure 5.19: Blue: spectrum of the input beam to the second multipass amplifier. Green: output spectrum of the amplified beam. Dashed line: simulations of the spectrum (see chapter 2).

crystal: about $7 \%$ of the radiation is unabsorbed and if concentrated on a small beam it can cause damage in optics. The beam profile of the four pump beams measured with a camera at the position of the crystal is shown in figure 5.17.

The major issue in the development of this amplifier proved to be thermal lensing. The total power pumped into the Ti:Sa can reach $100 \mathrm{~W}$ and about $31 \%$ of this quantity is converted to heat that must be dissipated from the crystal. The focal length of the crystal was measured using the same method as for the first multipass, resulting in $2 \mathrm{~m}$ at a cooling temperature of $-20{ }^{\circ} \mathrm{C}$ and $1.2 \mathrm{~m}$ at $15{ }^{\circ} \mathrm{C}$.

Although cooling the crystal to $-20{ }^{\circ} \mathrm{C}$ helped in decreasing the power of thermal lensing, the anti-reflection coated windows of the vacuum chamber could not resist the intensity of the Ti:Sa beam and were slightly damaged, which affected negatively the performance and quality of the laser beam. This event forced us to work with a cooling temperature of $15{ }^{\circ} \mathrm{C}$ for the rest of the experiment.

The beam profile for each pass is pictured in figure 5.18. The deformation of the beam profile as well as the increase in size during amplification is evident in the near-field beam profile after the third pass (figure 5.18(3)). It has lost its gaussian shape and resembles a combination of the pump profiles seen in figure 5.17. While this beam has preserved a reasonable quality, if the diameter of the pump beam 
was decrease, apart from an increase in the output energy, the output beam profile contained some hot-spots that damaged some of the following optics, implying that the damage limit was. The output beam after the third amplifier had a diameter of $5.3 \mathrm{~mm}$.

The output spectrum is pictured in figure 5.19. The effect of gain narrowing and shifting is also present in this amplifier, which decreases its bandwidth from $18 \mathrm{~nm}$ to $16 \mathrm{~nm}$ and shifts its peak to $810 \mathrm{~nm}$. The results of the simulations show a similar behaviour but predicted a broader output spectrum.

\subsubsection{Compressor}

The output beam of the second multipass amplifier is guided to the compressor by a series of mirrors and a telescope that enlarges the beam to a diameter of about $20 \mathrm{~mm}$.

The compressor of this laser is based on a Treacy design made with two gratings, similar to those in the stretcher of figure 5.20. The beam enters the compressor and makes a pass through two gratings and then a roof mirror changes the height of the beam so that it makes two additional passes through the gratings and the beam is compressed. The distance between the gratings was initially set to $18 \mathrm{~cm}$; however the gratings had to be separated an extra distance in order to compensate for the GDD introduced by all materials in the amplifier chain.

The pulse duration was measured with a Wizzler (Fastlite), which uses the third-order technique of self-referenced spectral interferometry [67]. A feedback loop can be set up between this device and the spectral shaper located before the regenerative amplifier in order to minimize the dispersion, especially third order, which was found to be the term which contributed the most to aberrations in the spectral phase. The pulse duration was determined to be $45 \mathrm{fs}$ and the spectral bandwidth retrieved was $19 \mathrm{~nm}$ FWHM, close to the 42 fs of the Fourier transform limited pulse, as seen in figure 5.21(a).

The first rough alignment of the compressor is performed by placing a converging lens and focusing the laser on air. The intensity of the laser will be enough to create plasma, then the distance between the gratings is changed until the maximum intensity is reached, which is where the compressor is aligned. Then the angle of 


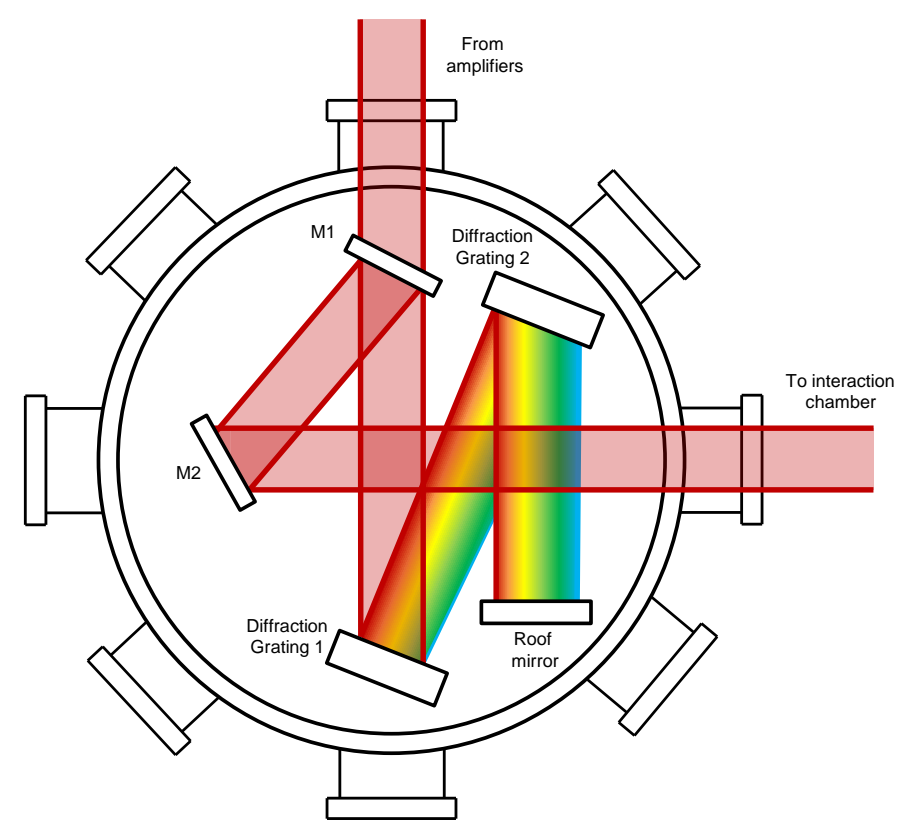

Figure 5.20: Scheme of the pulse compressor. M1 and M2 are two 2' high reflective mirrors. The pulse is compressed by two diffraction gratings and a roof mirror changes the height of the beam.

the gratings must be tuned with the help of the Wizzler in order to minimize third order dispersion. A feedback loop between both devices is used to fine tune second order dispersion.

The contrast of the pulse can be measured with the Wizzler up to the dynamic range of $300 \mathrm{fs}$ and is pictured in figure 5.21(b). The contrast at picosecond scale must be measured with a third order interferometer but we estimated it using a photodiode.

The efficiency of the compressor is $65 \%$. Measuring the efficiency of the compressor requires knowledge of the laser energy before and after the compressor. For peak powers exceeding 2 TW, the high intensity of the laser makes it prone to induce non-linear effects if it is outside of vacuum. Coupled to its high energy per pulse, a direct measurement using a power meter inside the vacuum chamber would probably result in damage. Therefore, in order to measure the efficiency, we placed the power meter after the compressor at atmospheric pressure but limiting the energy per pulse to $100 \mathrm{~mJ}$ (before the compressor) and calculating the ratio of transmitted energy to the input. 


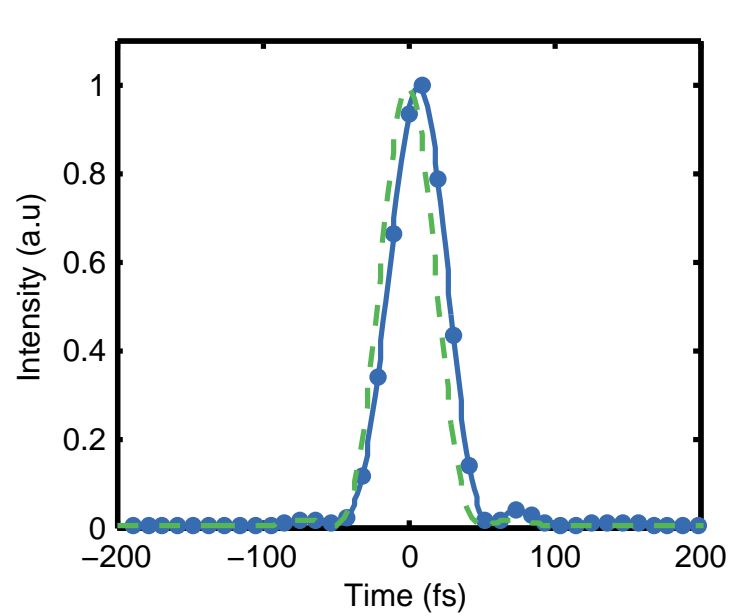

(a)

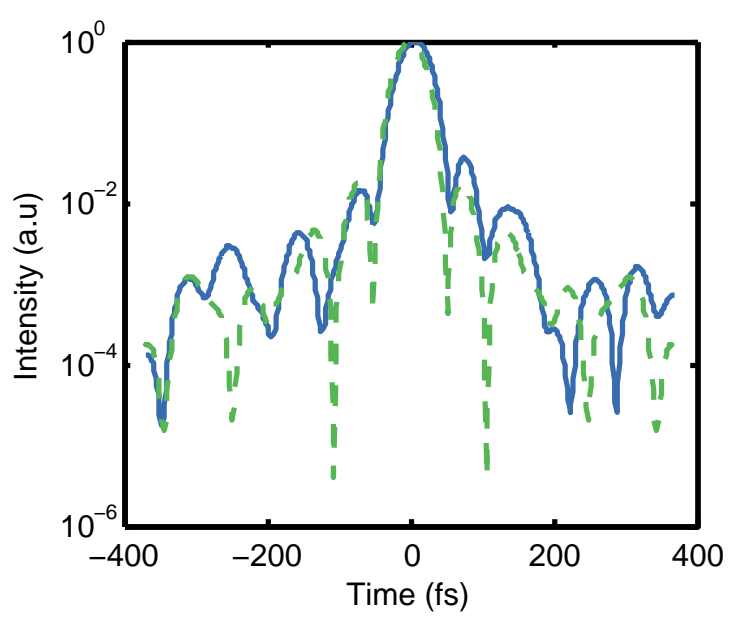

(b)

Figure 5.21: (a) Measured pulse duration. The dashed curve is the Fourier-transform limited pulse. The solid curve represents the pulse duration corresponding to $45 \mathrm{fs}$ FWHM. (b) Measured pulse duration in logarithmic scale.

After the compressor, the beam enters the interaction chamber where the proton acceleration experiments are performed. These experiments will be explained in detail in the next chapter.

\subsection{Improvement of contrast}

The concept of contrast, explained in section 2.4.2, has low impact on the performance of the laser, but can be very important to the efficiency of certain applications.

ASE is relevant for high aperture crystals; the Ti:Sa crystals used in the amplifiers are relatively small and are pumped with a moderate fluence of less than $2 \mathrm{~J} / \mathrm{cm}^{2}$. We tracked the source of contrast issues to the regenerative amplifier; pulses are coupled out of the cavity by a polarizer, but this element has small losses $(\sim 1-2 \%)$, and every roundtrip a leaked pulse exits the cavity separated by a roundtrip time, generating a train of pre-pulses of varying intensity.

A solution was found in the use of saturable absorbers. These devices are opaque to light until they saturate at a certain intensity and become transparent, which can clean the ASE pedestal and diminish the intensity of prepulses. Two saturable absorbers were included in the system. 


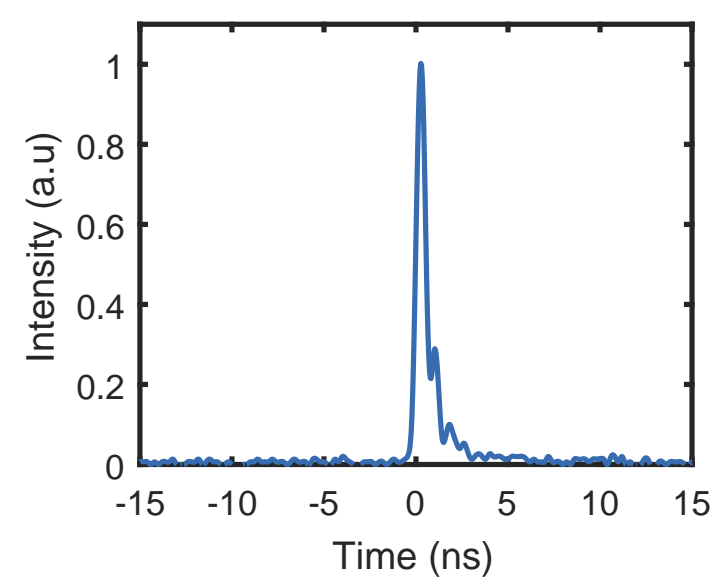

(a)

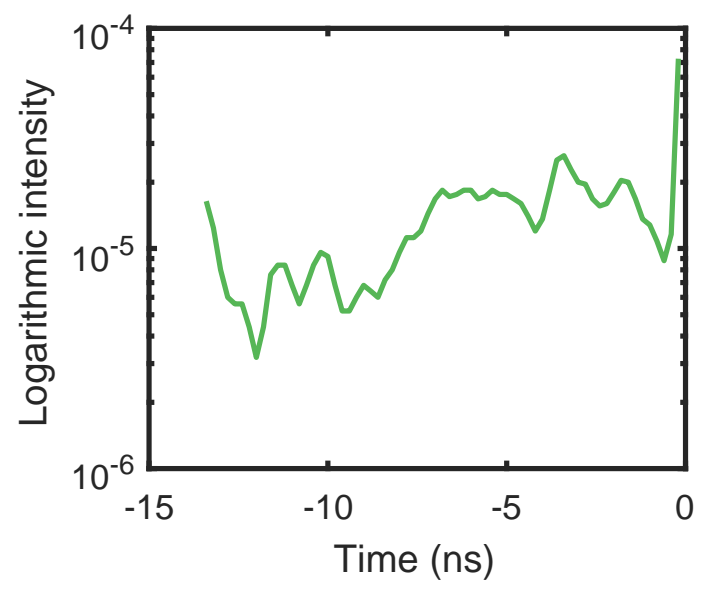

(b)

Figure 5.22: (a) Temporal profile of the Ti:Sa pulse before compression when measured by a fast photodiode. (b) Logarithmic scale of the temporal profile of the Ti:Sa pulse before compression. The main pulse is saturated.

The contrast of the pulse was estimated at the nanosecond scale using a fast photodiode. This method has a limited intensity and temporal resolution but it was used because there was no third-order autocorrelator in the laboratory. Figure 5.22 (a) represents the temporal profile of the stretched pulse, no pre-pulses or postpulses are observed. In order to increase the dynamic range of the measurement and distinguish the structure present in figure 5.22(b) the main pulse had to be saturated, which adds a layer of uncertainty to the calculated value of contrast.

There are other methods for the improvement of contrast, such as cross-polarized wave (XPW) and plasma mirrors. All methods for the improvement of contrast require some amount of energy losses. The use of an XPW device would require a whole new front-end of the laser and a plasma mirror, which is installed after the compressor, would decrease the final energy of the laser. The advantage of saturable absorbers with respect to plasma mirrors is that the loss of energy takes place while the pulse is stretched, therefore it can be compensated by an amplification stage. 


\section{Chapter 6}

\section{Laser acceleration experiments}

This work has been devoted until now to describe the development of a high average power few TW ultraintense laser, however, the ultimate goal of this thesis was to build a table-top laser accelerator. The source of protons and other particles is housed inside a small vacuum chamber that is attached to the compressor and surrounded by different nuclear detectors, requiring only very localized radioprotection. The combination of the TW laser and the interaction chamber creates a particle source that fills an area of less than $20 \mathrm{~m}^{2}$.

This chapter reports on the production of protons from a table-top Ti:Sa laser in single-shot operation. It starts with a brief introduction to the interaction of the ultraintense laser pulse and a material explaining particle acceleration mechanisms, followed by a description of nuclear detectors. Finally, the results of the proton acceleration experiments are presented and discussed.

\subsection{Proton acceleration mechanisms}

The interaction of the very intense electromagnetic field created by a focused laser can accelerate charged particles. When the intensity of a laser is very high, typically at $10^{17} \mathrm{~W} / \mathrm{cm}^{2}$ for near IR lasers, the velocity of the particle is very high and couples to laser magnetic field. For intensities beyond $10^{18} \mathrm{~W} / \mathrm{cm}^{2}$ for near IR lasers, electrons move at relativistic speeds and the motion equations need to be relativistic.

In the case of a propagating linearly polarized laser field the relativistic dynamics 


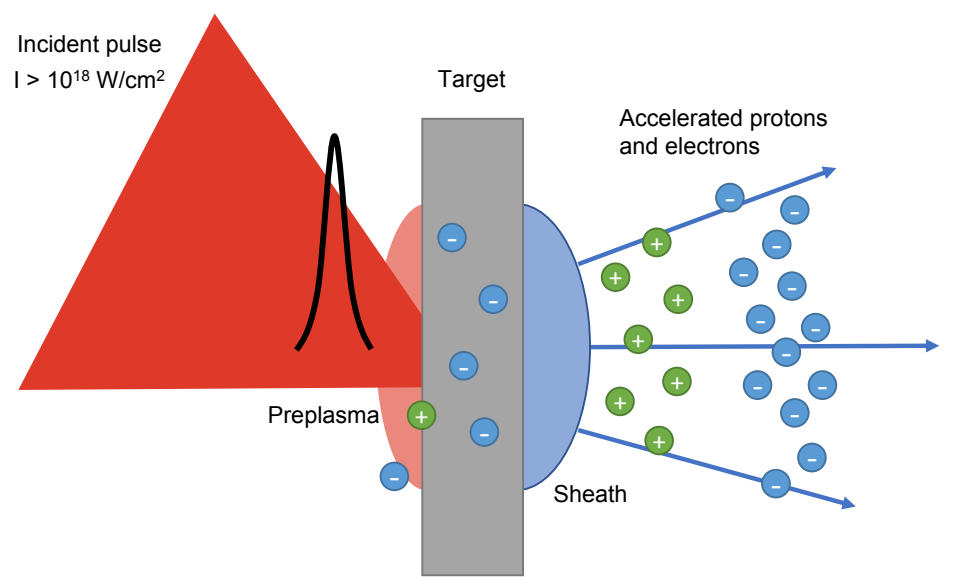

Figure 6.1: Sketch of the TNSA mechanism. The laser impinges generally at an angle on the target and creates a plasma that accelerates electrons followed by an acceleration of the protons by a capacitor-like interaction at the rear face of the target.

allows analytical solutions that were first published by Sarachik and Schappert [68]. The calculation seems complicated but the solution is very clear. The maximum energy that the particle gets inside a linearly polarized laser field is:

$$
E_{\text {max }}=m c^{2}+\frac{1}{4} \frac{q^{2}}{m w_{L}^{2}} I
$$

where $q$ and $m$ are the particle's charge and mass, $w_{L}$ the laser frequency and $I$ the intensity. Two things can be observed; the first is that the maximum energy of the particle increases as the laser frequency diminished; the second observation is that the more massive a particle is, the less it will be accelerate. Since a proton is 1836 times heavier than the electron, its acceleration by the laser field will be much less than for electrons or other lighter particles.

The acceleration of protons or ions can be achieved as a secondary effect typically in thin foil targets or gas jets as a consequence of the coupling of the laser and a plasma. A plasma is composed of quasi-free electrons and heavy ions, the first react to the electromagnetic of the laser while the ions are considered to be a static charge distribution due to their mass. In a plasma, highly non-linear collective effects dominate the phenomenology.

The interaction between the laser and the plasma depends on the electron 
density through the plasma frequency, a measure of the oscillations of electrons around ions:

$$
\omega_{p}=\sqrt{\frac{q_{e}^{2} n_{e}}{\epsilon_{0} m_{e}}}
$$

where $n_{e}$ is the electron density and $\epsilon_{0}$ the permeability of vacuum. The electron density that corresponds to a plasma density equal to the laser frequency is called the critical density. It separates two regimes of interaction of the laser with a plasma. If the laser frequency is below the plasma frequency, the situation is called underdense and the plasma behaves like a common medium where the laser can propagate freely. The overdense regime is reached when the laser frequency exceeds the plasma frequency and the laser is absorbed.

The most studied mechanism and the one that fits better with our experimental conditions is Target Normal Sheath Acceleration (TNSA). In TNSA, a high intensity laser impinges on the front surface of a thin foil, suddenly ionizing atoms and generating a current of hot electrons. About 10-50\% of the laser energy is transferred to the electrons [69] and are accelerated in direction of the density gradient or in direction of the laser propagation. As they traverse the foil, the electrons interact with ions or other cold electrons, suffering scattering and generating $\mathrm{x}$-rays and other types of particles. When the electrons reach the back surface of the foil, creating a negatively charged cloud (the sheath) during a very short time (picoseconds) at the other side of the target while the target is positively charged due to charge imbalance, as pictured in figure 6.1. The electron distribution and the positive charge of the target create a capacitor that generates an electric field of the order of $10^{12} \mathrm{~V} / \mathrm{m}$, strong enough to ionize atoms present at the back surface of the target, covered with a naturally present contamination layer. This layer is made of hydrocarbon and water molecules or additionally deposited material and represent the source of the protons or ions accelerated. The ions are accelerated perpendicular to the surface of the target since the electric field is also normal to the target, independent of the incidence direction of the laser. 


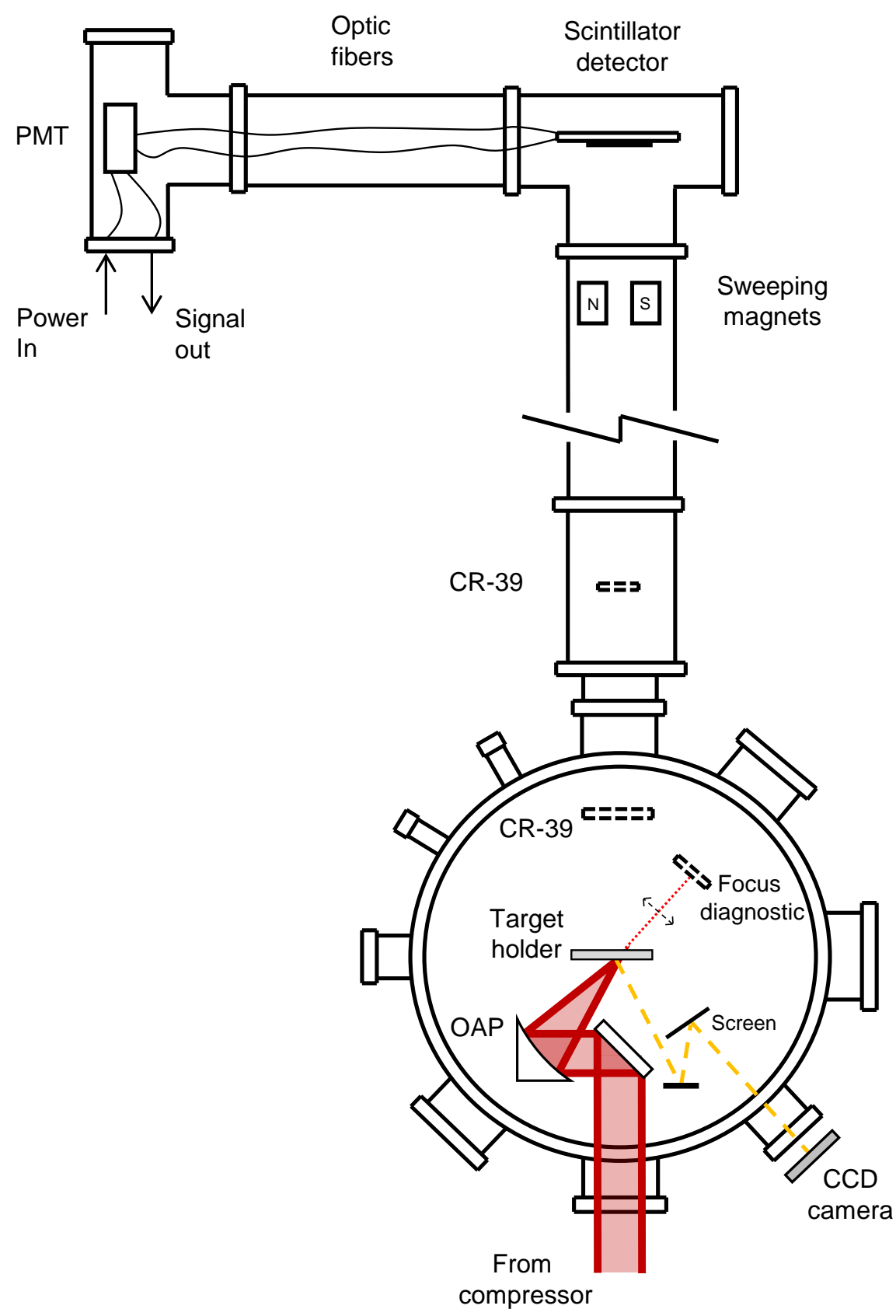

Figure 6.2: Scheme of the proton acceleration experiment. In the first vacuum chamber a mirror steers the laser beam onto the OAP (off-axis parabola), which focuses the laser onto a target where the protons are generated. Also, pictured in the image there are several possible positions of the passive radiation detectors (CR-39). At the end of the flight tube there is a scintillator detector coupled by fiber to a photomultiplier tube (PMT). 


\subsection{Experimental setup}

The proton acceleration experiments take place in a vacuum chamber situated after the compressor. The experiments are performed when the pressure reaches at least 20 mTorr. A pulse coming from the compressor is focused by and off-axis parabola (OAP) into a solid target located at the centre of the vacuum chamber. The target holder is connected to three motors that supports the quick refresh of the foils and the fine tuning of the position of the target with respect to the laser focus. The laser focus can be imaged, in absence of the target, using an aspheric lens attached to a CCD camera. The off-axis parabola is aligned using the size of the focus as a figure-of-merit. Meanwhile, in order to verify the correct alignment of the target with respect to the laser focus, the setup includes another CCD camera and a screen inside the vacuum chamber.

The protons are originated in the rear side of the target, hence the particle detectors are situated perpendicular to the target holder. Inside the same vacuum chamber, some passive detectors such as CR-39 chips or radiochromic films can be placed. As an additional detector, a scintillator crystal is situated at the end of a 2.27 meters long time-of-flight tube coupled to the vacuum chamber. This detector can provide a live signal in an oscilloscope. This scheme is pictured in figure 6.2.

\subsubsection{Target holder}

The target holder (figure 6.3) was designed to be used in single-shot configuration. It can hold up to 24 single targets divided into two vertical rows. Three motors can move the targets in three degrees of freedom: the height can change the row of the targets and the other two position the target in focus. The target holder is not positioned perpendicularly to the beam reflected by the off axis parabola, so two of the motors are necessary to position the target with respect to the focus while keeping the beam centred in the metal foil. They can move in single steps of $2.5 \mu \mathrm{m}$, so for an angle of incidence of $30^{\circ}$, the precision of the positioning of the target is about $3.5 \mu \mathrm{m}$. All these features allow for testing several targets without breaking vacuum.

The targets used in this experiment are thin foils of aluminium and aluminized mylar of various thickness. 


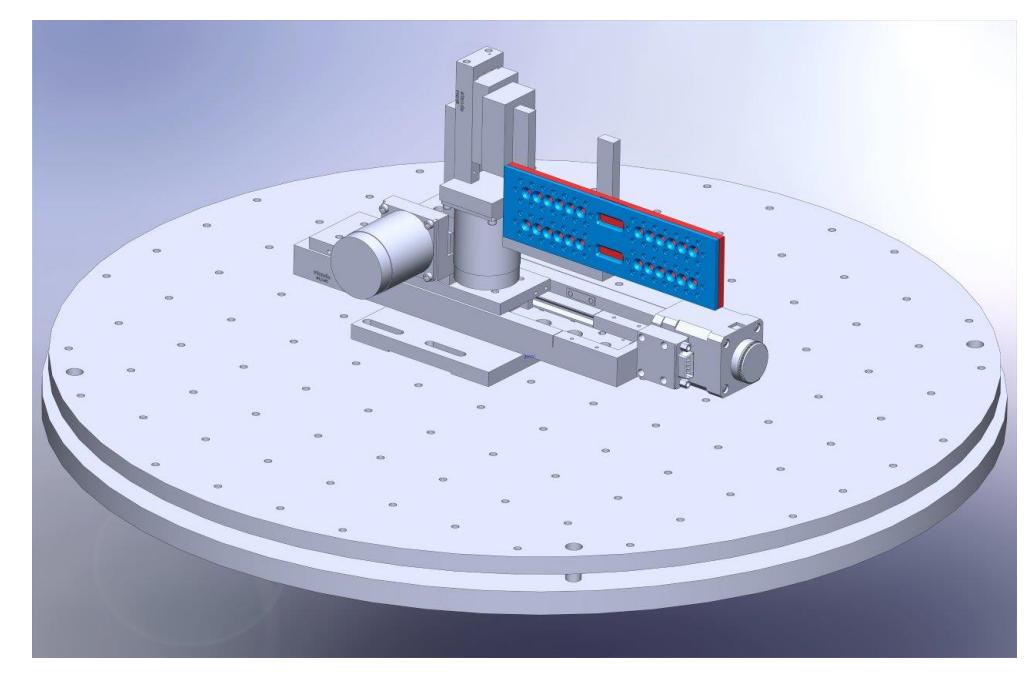

Figure 6.3: Scheme of the target holder system. The individual targets are housed in the target holder (blue) in two rows of 12 units each. Three motors can move the target with micrometric precision in the three axis.

\subsubsection{Focusing and target positioning}

In proton acceleration experiments, the flux and maximum kinetic energy of the protons is highly dependent on intensity. This means that tight focusing and reliable positioning of the target are crucial to ensure the success and repeatability of the experiments. Therefore, one of the most important tasks in the experiment is to characterize the focal spot of the Ti:Sa laser.

The beam of the TW laser is focused through an off-axis parabola with a numerical aperture of $\mathrm{f} / 3$ and $100 \mathrm{~mm}$ of focal length. The measurement of the focal spot size in this experiment is complicated as the high intensity of the laser requires an extreme attenuation of the laser to a few microjoules or less while preserving thermal lensing in the power amplifiers. This was achieved by enlarging the distance between the seed Ti:Sa pulse from the pump pulses and adding several neutral density filters after the last telescope before the compressor.

Once the beam is properly attenuated, a CCD camera can image directly the focal spot of the Ti:Sa laser. The camera used was a WinCamD-UCD12 ${ }^{1}$; The pixel size of the CCD sensor is $4.65 \mu \mathrm{m}$ which is of similar size as the beam we would like to image, which is an unacceptable resolution. Therefore, in order to improve the resolution we used an optical setup consisting of an aspherical lens of

\footnotetext{
${ }^{1}$ DataRay, USA, dataray.com/
} 


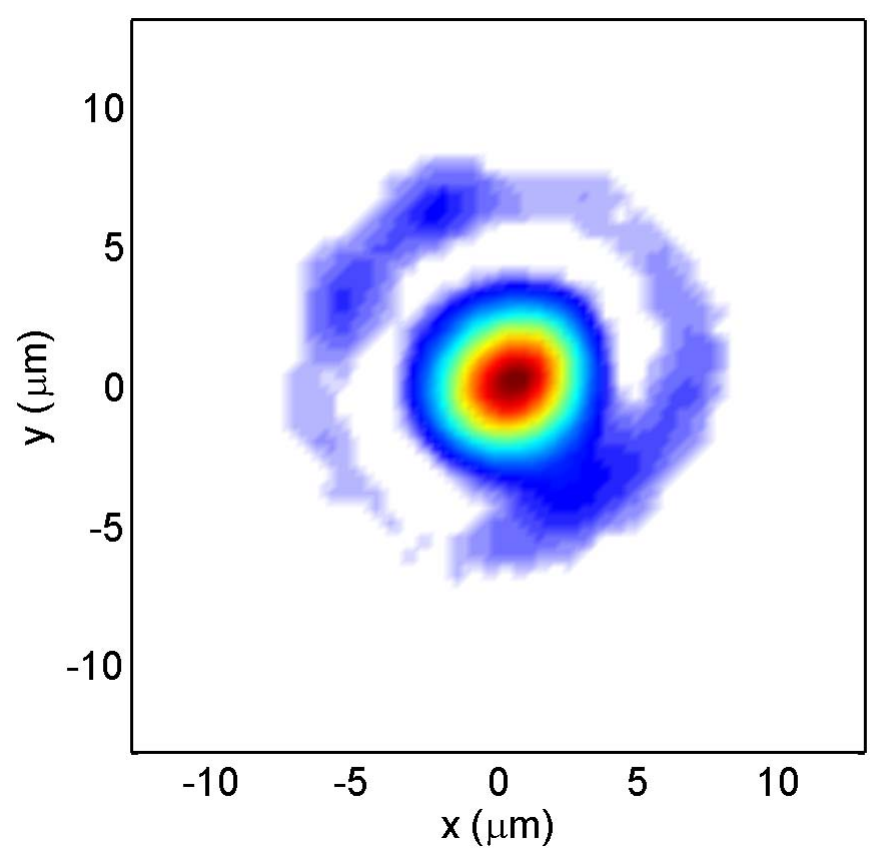

Figure 6.4: Image captured a CCD camera of the focal spot of the He-Ne laser when focused with a $100 \mathrm{~mm}$ focal length off-axis parabola.

a very small focal length $(7 \mathrm{~mm})$ attached to a tube of $50 \mathrm{~mm}$ length which was in turned coupled to the CCD camera. This setup provides a magnification of 8.2. The camera and the attached setup are mounted on a translation stage that can be easily removed and is only installed back onto the vacuum chamber whenever the measurement of the focal spot size is needed.

As an auxiliary beam, we used the $633 \mathrm{~nm}$ light from a collimated He-Ne laser that follows the same path as the TW laser through a flip mirror just before the last magnifying telescope before entering the compressor. This laser is very convenient as an alignment beam for all the optical elements inside the chamber as a result of its visibility and excellent beam quality. One of the most critical tasks, the alignment of the off-axis parabola was done while imaging the focal spot of the He-Ne with the camera as described above. Then the two degrees of freedom are adjusted iteratively until the best focus is achieved. The resulting focal spot of the He-Ne beam can be seen in figure 6.4. Then the auxiliary beam is removed and the imaged Ti:Sa laser focus is optimized with some finishing adjustments by the off-axis parabola. 

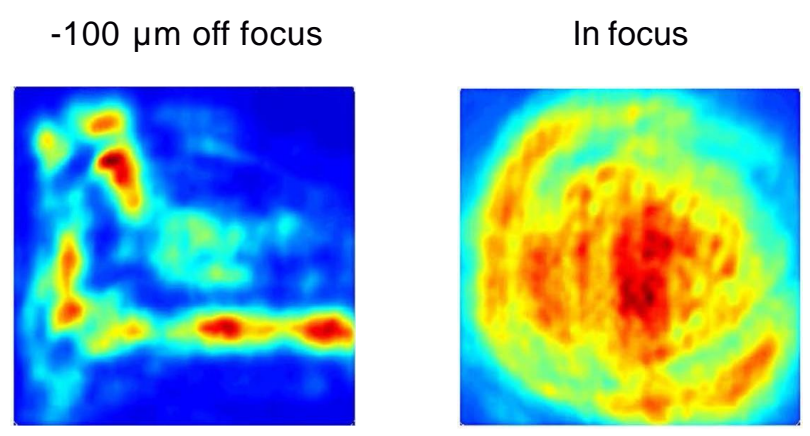

$+100 \mu \mathrm{m}$ off focus

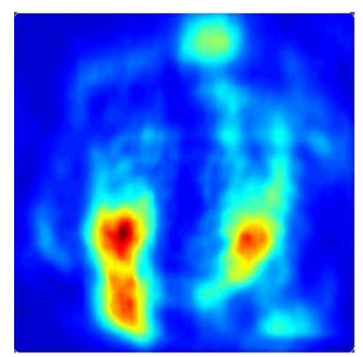

Figure 6.5: Images of the speckle pattern generated by the front surface of the target for several distances of deviation from the laser focus.

Once the off-axis parabola is adjusted and the focal spot of the Ti:Sa laser characterized, the next task is the arrangement of the positioning of the target with respect to the reference of the focus of the laser. The accuracy of this positioning must be less than the Rayleigh length of the laser (the region where the beam is considered to be focused). For a gaussian beam, introducing similar parameters to the setup of this experiment, the Rayleigh length $z_{0}=\pi w_{0}^{2} / \lambda$ is roughly $50 \mu \mathrm{m}$.

However, the relative positioning of the Ti:Sa focus and the target surface is not a trivial job. First, the Ti:Sa laser beam cannot be used for this task because although attenuated, it may still interact with the target burning a hole or degrading its surface, which would be detrimental for the actual experiment. Additionally, the target foils are so thin that any mechanical contact is likely to break or induce some deformation on the surface. Taken all this into consideration, the method used in this experiment to track the position of the focal spot with respect to the target is to image the backscatter light from the target [70].

As the light source we used a He-Ne laser that follows the same path as the Ti:Sa laser. The scattered light from the front surface of the target is projected onto a screen inside the vacuum chamber and is then imaged by a CCD camera (which an attached telescope) through a viewing port. This retro-reflected light forms a speckle pattern that is just the interference of the beam with itself in a diffusive surface, therefore the smaller the surface illuminated the more uniform this pattern will become. An example of this behaviour can be seen in figure 6.5. It is important to note that the speckle can only be manifested on targets that 
have a surface roughness higher or close to the scale of the laser wavelength. The advantage of this technique is that it can be done for every single target without breaking vacuum, even visually in a short period of time; however it can not be used in a multi-shot experiment especially when the laser has a repetition rate of $100 \mathrm{~Hz}$, as the diagnostics would not be able to work at such high rate.

Still, with this method the focus of the He-Ne can be situated on the surface of the target but there is no assurance that the focus of the He-Ne and the Ti:Sa laser are at the same position. Both lasers will only be focused on the same position if their divergence is similar at the entrance to the off-axis parabola. To fulfil this condition, a magnifying telescope is installed close to the He-Ne with one of the lenses mounted on a translation stage. Moving this lens will change the divergence of the He-Ne and be made to match the focus position of the Ti:Sa laser.

\subsection{Proton detection experiments}

The primary goal of these experiments was to detect protons from the interaction of the laser with a solid thin target. However, the detection is not trivial especially in the case of laser plasma acceleration as the protons are joined by electrons, ions, energetic photons and an intense low-frequency electromagnetic pulse, hence in a poorly designed experiment the detection of the protons would be masked by the signal generated by all of the other sources. In this experiment we used several proton detectors, developed in the Instituto de Instrumentación para la Imagen Médica, I3M (Valencia), that will be detailed below, in order to reduce any observational error in the measurements.

\subsubsection{CR-39}

CR-39, allyl diglycol carbonate, is a plastic polymer used by optometrists to make glasses as it has good optical properties and is especially resistant to scratches. We used it in the experiment because it has the advantage that it is insensitive to electrons or photons, so only protons or ions can be detected. It is an easy component to set up in the experiment but the process of etching is tedious and does not provide a live detection of radiation [71]. 
This plastic works as a detector because when an energetic proton arrives at the chip, it destroys the links between polymer chains through its range of penetration. However, these damaged tracks are not visible and the CR-39 must be treated with a heated basic solution which erodes the volume damaged by the radiation and pits are formed. The energy of the particles can be inferred from the shape and size of the pits, provided they are below the maximum energy of approximately 7 $\mathrm{MeV}$ for protons.

The sets of $1 \mathrm{~cm}^{2}$ CR-39 chips used in this experiment were provided by Radosys $^{2}$ They were calibrated with proton beams of $0.1-5.5 \mathrm{MeV}$ at the National Accelerator Center (CNA), Seville. The etching process was carried out with a bath of $6.25 \mathrm{M} \mathrm{NaOH}$ at $90^{\circ} \mathrm{C}$ for 4 hours. In these conditions the relation between size and energy of the particles grows monotonously up to $1 \mathrm{MeV}$ and then decreases, so there may be ambiguities in the characterization of energy. In order to solve this, thin aluminum foils of 4 and $7 \mu \mathrm{m}$ where used to attenuate the energy of the protons and expand the dynamic range of measurement beyond $1 \mathrm{MeV}$.

Any sort of laser effect on the CR-39 is not reported in literature. However, we made sure that no chip was positioned in the direction of the laser beam, before or after the target. Also, as mentioned before, in this experiment all the CR-39 chips were covered with Aluminum foils, which reflects most of the light in the visible and near infrared regions.

\subsubsection{Radiochromic films}

Radiochromics (RCF) are films which have one or more sensitive layers (a few tens of microns thick) that polymerize when they are exposed to ionizing radiation. The polymerization process creates a dye that changes the optical properties of the film, so a simple optical density scan will directly retrieve the dose deposited so it is a very useful tool to measure the spatial distribution of radiation. Unlike CR-39, radiochromic films are sensitive to electrons and photons as well as protons and ions.

In the case of protons, the dose deposited in the film is strongly dependent on its initial energy: if the Bragg peak falls within the active area a strong signal is

\footnotetext{
${ }^{2}$ Radosys, Hungary, radosys.com
} 


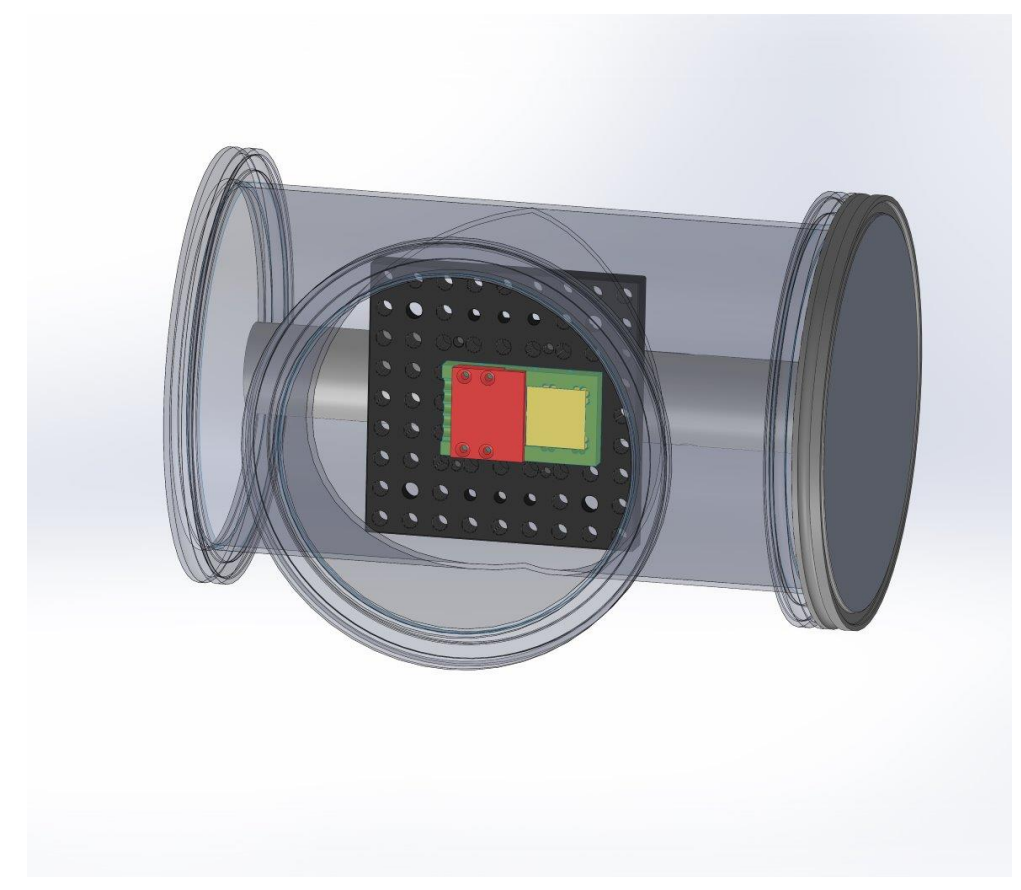

Figure 6.6: Drawing of the scintillator detector used in the experiments. The yellow surface houses the scintillator crystal.

registered but if it falls beyond, then the signal will be much smaller although that particle was more energetic. For this reason it is usual to stack several radiochromic films to measure the proton dose and spatial distribution at different energies.

In this experiment it was noticed that the part of the laser beam that was not absorbed in the target could develop the radiochromic film if proper care was not taken to remove the film from the laser path after the target.

\subsubsection{Scintillator and time-of-flight}

The principal particle detector used in this experiment is a scintillator detector situated at the end of a time-of-flight tube. Unlike CR-39 and radiochromic films, this setup enables a real time measurement of the energy and number of particles detected and does not require breaking vacuum between shots. The scintillating material is an organic plastic with an effective area of $25 \times 25 \mathrm{~mm}^{2}$ and a thickness of $5 \mathrm{~mm}$ as seen in figure 6.6. It works by emitting photons when excited with ionizing radiation; this light is guided by a set of optical fibers to a photomultiplier tube (PMT) which generates a signal that can be recorded with a high bandwidth oscilloscope that can resolve very short time events. 
Time-of-flight (TOF) is a method that refers to the measurement of the time an object takes to travel a predefined length. In the context of this thesis, the TOF is a technique for measuring the spectral distribution of different ion species. It involves separating the radiation source and the detector by some distance $\mathrm{L}$ in order to measure the time it takes for the ions to reach the detector. Taking into account that the velocity of protons depend on their kinetic energy, it allows for a time resolved energy spectrum [72].

Since in the interaction of the laser and the target x-rays and other energetic photons are generated, they arrive at the scintillator before all ions as they travel at the speed of light $\mathrm{c}$ and provide a signal. Then, assuming all radiation was generated at the same time, the detector will deliver another signal for ions a time $\Delta t$ later. Electrons do not mask the ion signal because they are deflected by a dipole magnet situated close to the scintillator. Thus, the velocity $v_{i}$ of the protons can be expressed as:

$$
\beta=\frac{v_{i}}{c}=\frac{1}{\frac{c \Delta t}{L}+1}
$$

And the kinetic energy of the protons is related to the velocity as:

$$
E_{i}=M_{i} c^{2}\left(\frac{1}{\sqrt{1-\beta^{2}}}-1\right)
$$

Where $M_{i} c^{2}$ is the rest mass of the ion species. The distance $\mathrm{L}$ between the target and the scintillator was designed to be $2.27 \mathrm{~m}$. The face of the scintillator is covered with a thin aluminium foil in order to avoid noise and possible damage by the light scattered from the laser and the entrance of light to the PMT is coupled through a neutral density filter in order to avoid saturation of the signal and increase the dynamic range of the measurements. The number of ions and therefore the absolute proton spectral distribution can be inferred from the height of the signal created by the PMT which was previously calibrated, together with the CR-39, at the $3 \mathrm{MV}$ cyclotron at the CNA in Seville [73], for three neutral filters with optical densities ranging from 1 to 3 . 


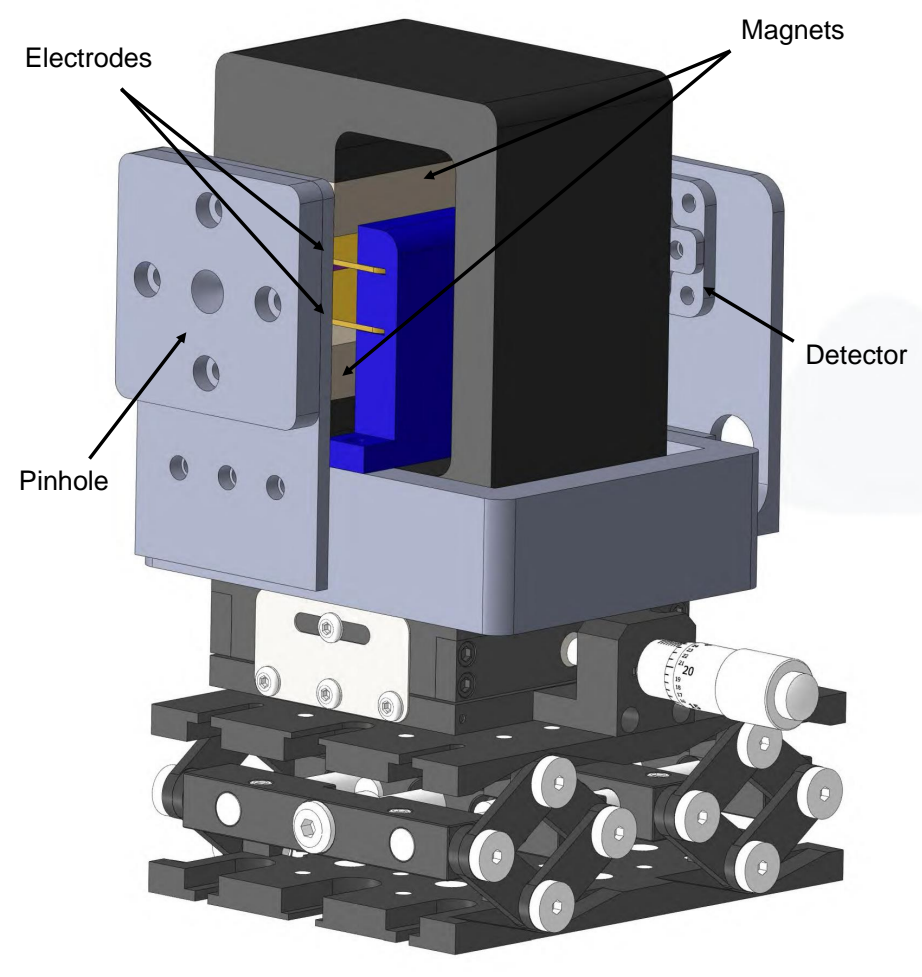

Figure 6.7: Schematic design of the Thomson parabola. The magnetic field is generated by a pair of magnets joined by an iron yoke while the electric field is generated by a pair of copper electrodes. The rear metallic plate supports the CR-39 chips. 


\subsubsection{Thomson Parabola}

A Thomson spectrometer or parabola is a device in which parallel electric and magnetic fields are superposed perpendicular to the path of incoming particles in order to induce a deviation of ions species according to their charge to mass ratio.

The electric field deflection scales with the charge-to-energy ratio of the incoming ions and the magnetic deflection is proportional to the charge-to-momentum ratio. After traversing the spatial zone where both fields are present, the resulting trajectories are collected at the detector plane by a detector, which is CR-39 in this particular design. The curvature of these traces depend on the charge-to-mass ratio of the ions, providing the spectroscopic information for all the species present in the beam.

The deviation of the different ions on the surface of the detector, under the assumption that the electric and magnetic fields are uniform and in the small angle approximation can be expressed as:

$$
\begin{aligned}
& \Delta x=\frac{q E}{m v^{2}} L\left(\frac{L}{2}+l\right) \\
& \Delta y=\frac{q B}{m v} L\left(\frac{L}{2}+l\right)
\end{aligned}
$$

where $\mathrm{q}, \mathrm{m}$ and $\mathrm{v}$ are the charge, mass and initial velocity of each ion; $\mathrm{E}$ and $\mathrm{B}$ are the amplitude of the electric and magnetic fields; $\mathrm{L}$ is the length where the magnetic and electric field are present and 1 is the distance from the exit of the magnets and electrodes to the detector plane. These equations are only valid for ions with trajectories perpendicular to the electric and magnetic fields, which is not the case for particles accelerated by the laser plasma interaction. In order to filter the ions that fulfil this requirement, a pinhole should be added at the entrance of the parabola.

The design of the Thomson parabola used in the experiment (figure 6.7) was successfully tested [74], however due to technical difficulties, in this thesis the electric field could not be used. The magnets were used only to filter electrons off the scintillator, in order to have a cleaner proton signal. Due to the higher charge-to-mass ratio of electrons they are strongly deflected but protons do not suffer much of a deviation from their initial path. Energetic electrons may not be 


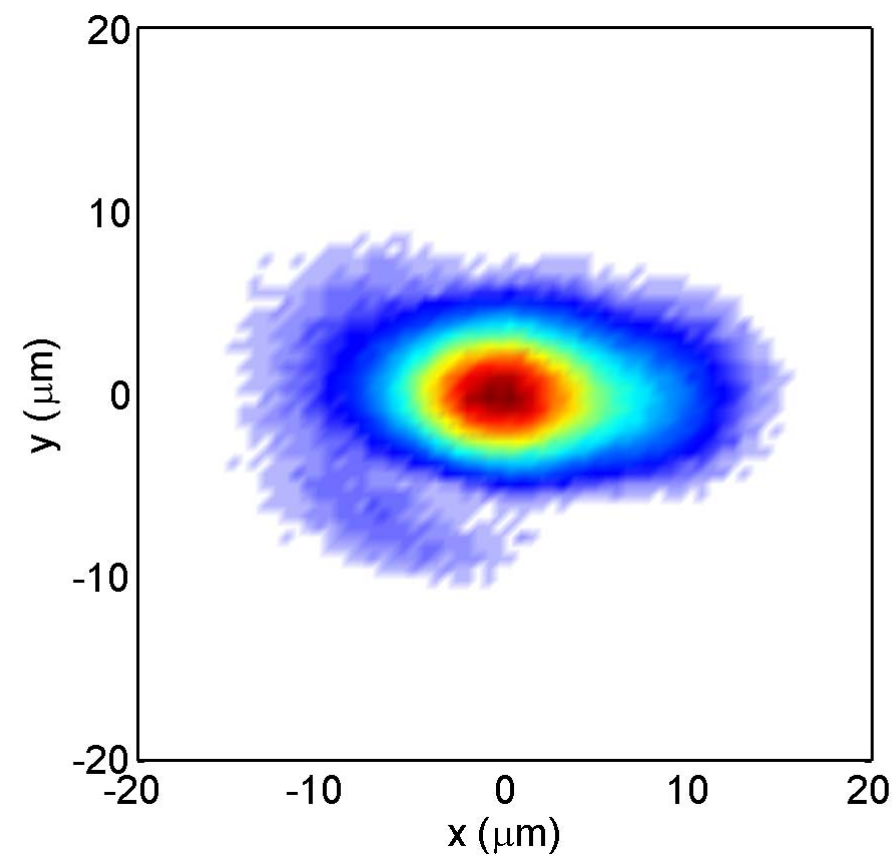

Figure 6.8: Image captured by a CCD camera of the focal spot of the Ti:Sa laser when focused with a $100 \mathrm{~mm}$ focal length off-axis parabola. Its size is $6.0 \times 10.1$ $\mu \mathrm{m}$ FWHM.

deflected but they do not contribute to the noise of the signal from the much slower protons.

\subsection{Experimental results}

The ultra high intensity laser developed in the previous chapters of the thesis was used as driver of proton acceleration using solid targets. The operating mode of the laser was modified to provide a single pulse when a trigger was actioned, since the target holder does not support the full repetition rate of the laser.

Two experimental campaigns of proton acceleration are reported in this thesis corresponding to a configuration of the laser with low energy per pulse and low contrast and another with higher energy and improved contrast (see section 5.3). These experiments were published in [75]. 


\subsubsection{Low contrast regime}

The laser had an energy of $153 \mathrm{~mJ}$ before the compressor which accounts for about $96 \mathrm{~mJ}$ after the compressor. The beam profile at the focus was measured directly with a camera and is pictured in figure 6.8. It has an elliptic shape that is probably due to the spherical aberration introduced in the mirrors that compensate the thermal lensing in the amplifiers. The short axis measured $6.0 \mu \mathrm{m}$ while the long axis measured $10.1 \mu \mathrm{m}$ FWHM or $9.7 \times 20.0 \mu \mathrm{m}$ when measure at a value of $1 / e^{2}$ of the peak intensity. It was calculated that $80 \%$ of the energy was enclosed in the $1 / e^{2}$ area. The peak intensity had a value of $1.2 \cdot 10^{18} \mathrm{~W} / \mathrm{cm}^{2}$.

During this experimental campaign, the only diagnosis of proton energies and numbers was performed using CR-39, as the TOF and the scintillator were not operative yet. An example of detected tracks for several targets can be seen in figure 6.9(a). The CR-39 chips were analyzed through a microscope and the size of the tracks correlated to the calibration in order to deduce the proton energy $E_{p}$. The number of protons $N_{p}$ was fitted to a Boltzmann distribution:

$$
N_{p}=\frac{N_{0}}{E_{p}} \exp \left(-E_{p} / k_{B} T\right) /\left(1+\exp \left(\left(E_{p}-E_{\max }\right) / \Delta E\right)\right)+N_{b g}
$$

where $\mathrm{T}$ is the temperature of the plasma, $k_{B}$ is the Boltzmann constant.

The spectrum of the accelerated protons can be seen in figure $6.9(\mathrm{~b})$. The results of this campaign yielded a maximum energy of $650 \mathrm{keV}$, found using a target of aluminium of $10 \mu \mathrm{m}$ thickness. The use of other aluminium targets of different resulted in less maximum energy while using a foil thinner that $7 \mu \mathrm{m}$ did not yield any accelerated protons distinguishable from background. The same result was observed in any of the Mylar foils; the few tracks that were observed were consistent with background levels. This result is most likely the consequence of the poor contrast of the laser.

The results of the experiment were consistent between shots, even though the vacuum chamber had to be opened to retrieve the CR-39 chips after each laser shot, without any additional adjustment of the setup, and could be reproduced even after a complete re-alignment of the setup. 
Al $7 \mu \mathrm{m}$

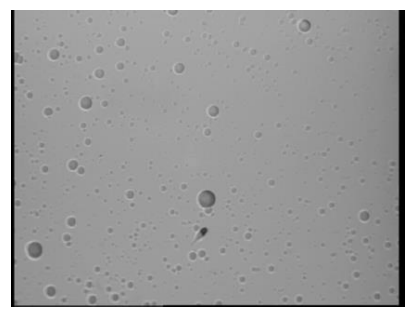

Al $18 \mu \mathrm{m}$

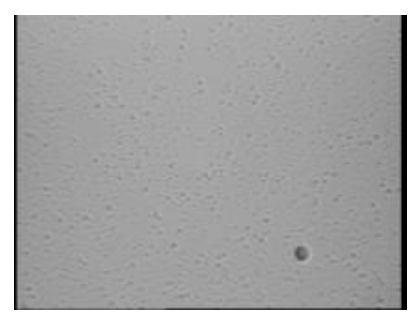

Al $10 \mu \mathrm{m}$

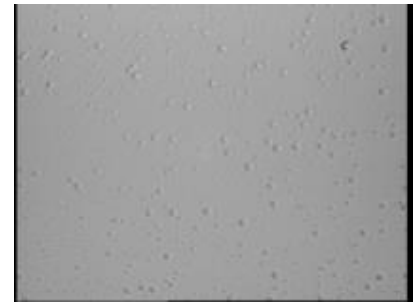

Mylar $6 \mu \mathrm{m}$

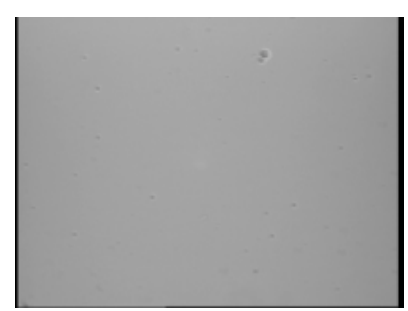

(a)
Al $12.5 \mu \mathrm{m}$

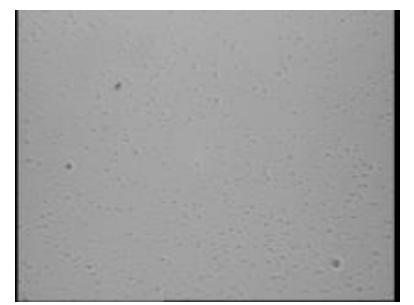

Mylar $13 \mu \mathrm{m}$

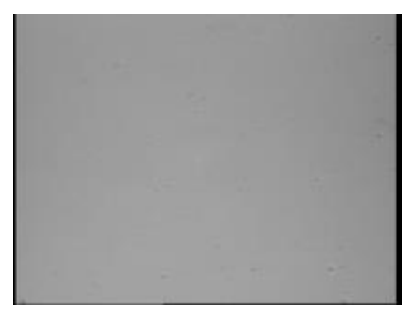

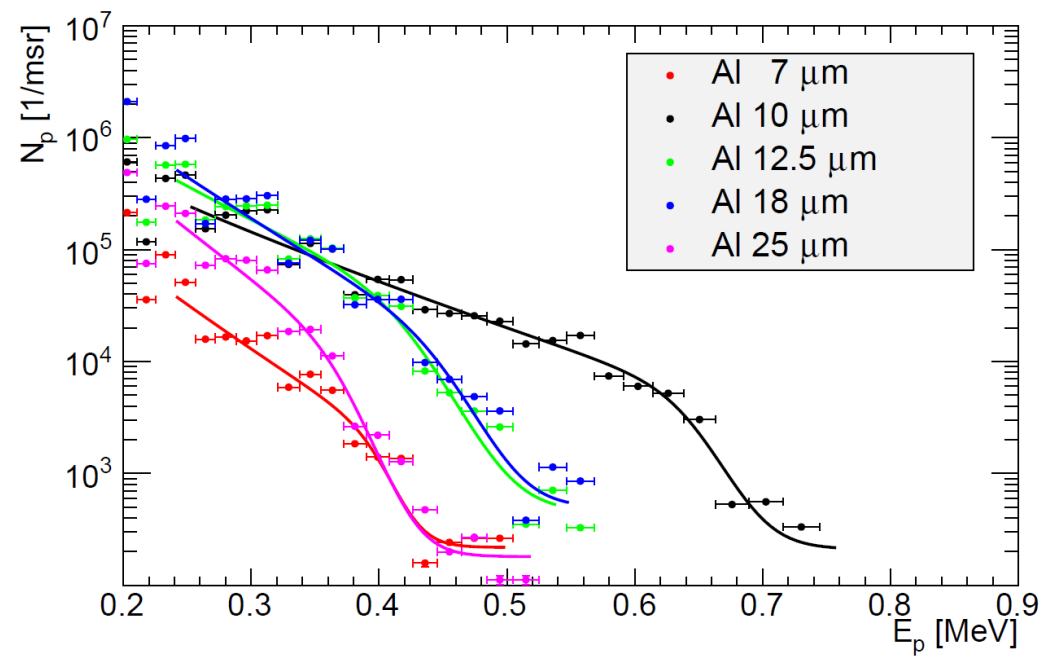

(b)

Figure 6.9: Experimental results from the low contrast experimental performed with $100 \mathrm{~mJ}, 45 \mathrm{fs}$ pulses and an intensity of $1.2 \cdot 10^{18} \mathrm{~W} / \mathrm{cm}^{2}$. (a) Image of etched CR-39 chips for several targets: aluminium from 7 to $18 \mu \mathrm{m}$ thickness and 6 and $13 \mu \mathrm{m}$ Mylar. (b) Spectrum of the protons accelerated in the low contrast regime extracted from CR-39 tracks for several targets of different materials and thickness. 


\subsubsection{High contrast regime}

The laser had an energy of $315 \mathrm{~mJ}$ before the compressor which results in about $195 \mathrm{~mJ}$ after the compressor. The beam profile at the focus was measured directly with a camera and is pictured in figure 6.10. Some of its elliptical shape could be reduced by reducing the working angle of some of the spherical mirrors present in the amplifiers. It measured $6.1 \times 8.3 \mu \mathrm{m}$ FWHM or a diameter $\left(1 / e^{2}\right)$ of $10.5 \times$ $15.4 \mu \mathrm{m}$. It was estimated that $78 \%$ of the energy was enclosed in the $1 / e^{2}$ area. The calculated value of peak intensity was $1.8 \cdot 10^{18} \mathrm{~W} / \mathrm{cm}^{2}$.

In order to observe the distribution of the protons and electrons generated in the interaction of the laser and the target, a radiochromic film was positioned at a distance of $20 \mathrm{~cm}$ from the interaction point and developed though five consecutive laser shots using five similar aluminium target foils. A central spot surrounded by a halo can be seen in figure 6.11, which represents the spatial distribution of the radiation generated. The straight lines are believed to be artefacts created by the laser light or the ultra-violet light generated by the interaction.

In this campaign the TOF and the scintillator were used as the principal detector while the CR-39 chips were used as a secondary source of information simultaneously; the resulting maximum energies match within $100 \mathrm{keV}$, the tracks can be observed in figure 6.12(a). The signal provided by the scintillator could be viewed at an oscilloscope. The signal displays two major contributions: first, a peak is detected that corresponding to electromagnetic radiation and high energy electrons travelling close to the speed of light, followed by a broader peak delayed by hundreds of nanoseconds that corresponds to the protons. This method allows for a complete characterization of the accelerated particles without the need of opening the vacuum chamber each shot. The energy spectra used for fitting the TOF data uses also the Boltzmann distribution (equation 6.7).

The spectrum of the accelerated protons measured by the TOF for several targets is presented in figure 6.12(b). A maximum energy of $1.6 \mathrm{MeV}$ was achieved using a $0.8 \mu \mathrm{m}$ thickness aluminium target and $1.2 \mathrm{MeV}$ using a $6 \mu \mathrm{m}$ thickness Mylar target. The improvement of contrast (detailed in section 5.3) is the major contribution to the increase in proton energy since it enabled the use of thinner targets. 


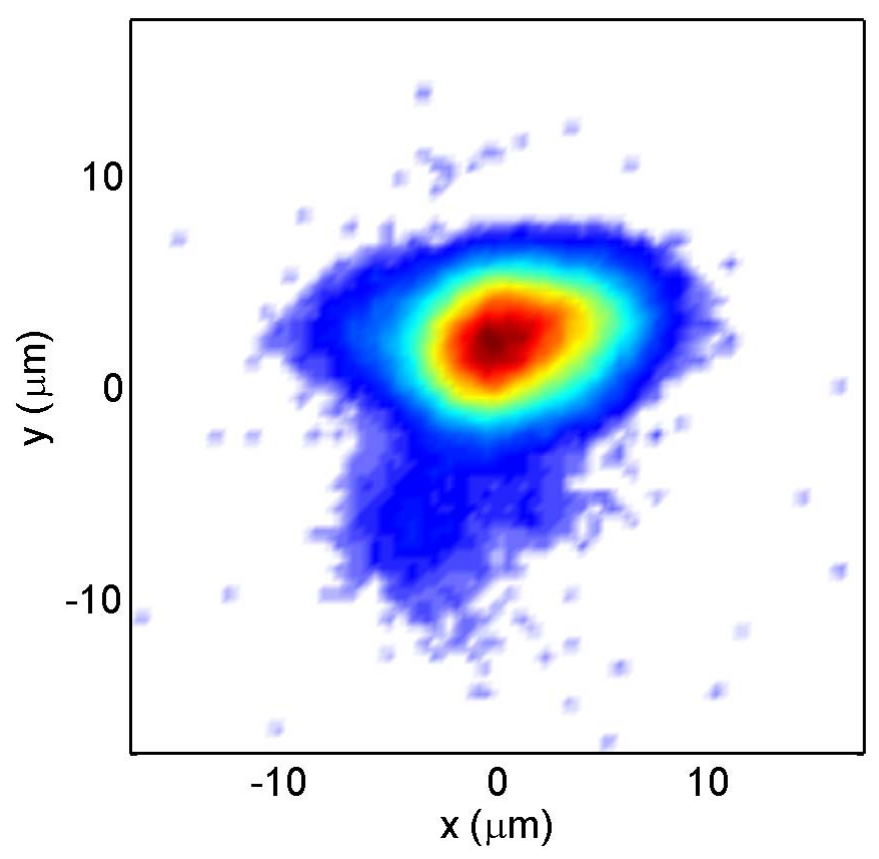

Figure 6.10: Image captured a CCD camera of the focal spot of the Ti:Sa laser when focused with a $100 \mathrm{~mm}$ focal length off-axis parabola. Its size is $6.1 \times 8.3$ $\mu \mathrm{m}$ FWHM

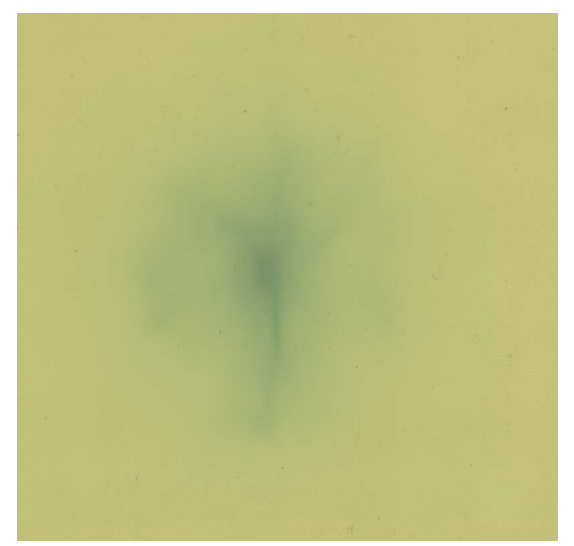

Figure 6.11: Scanned image of a RCF after being developed by five consecutive laser shots. 
Al $4 \mu \mathrm{m}$

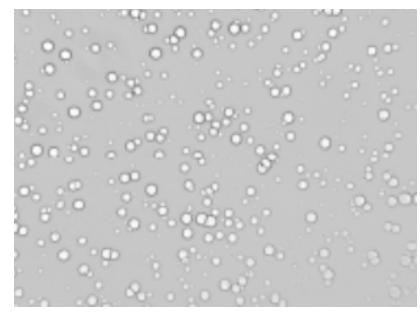

Al $18 \mu \mathrm{m}$

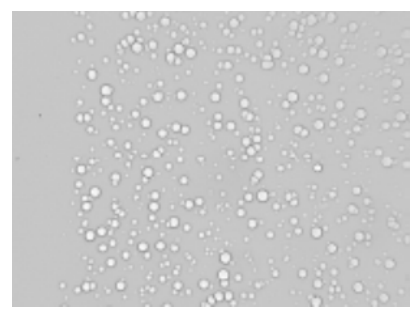

Al $7 \mu \mathrm{m}$

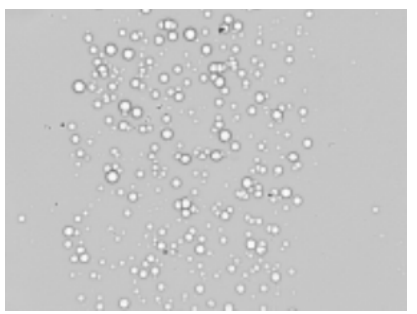

Mylar $6 \mu \mathrm{m}$

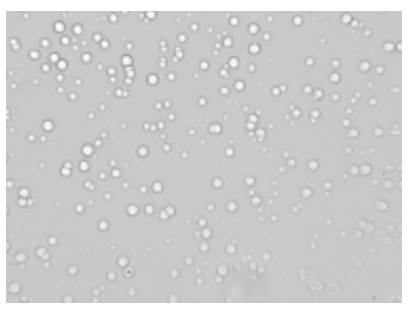

(a)

(a)
Al $10 \mu \mathrm{m}$

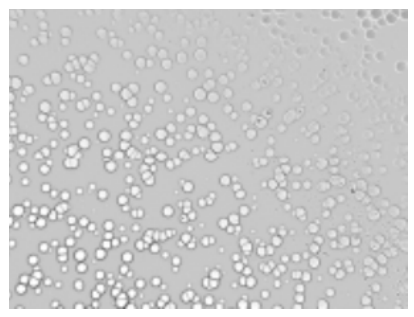

Mylar $13 \mu \mathrm{m}$

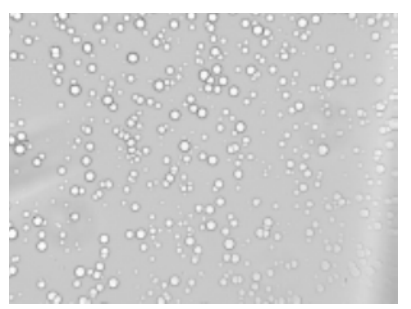

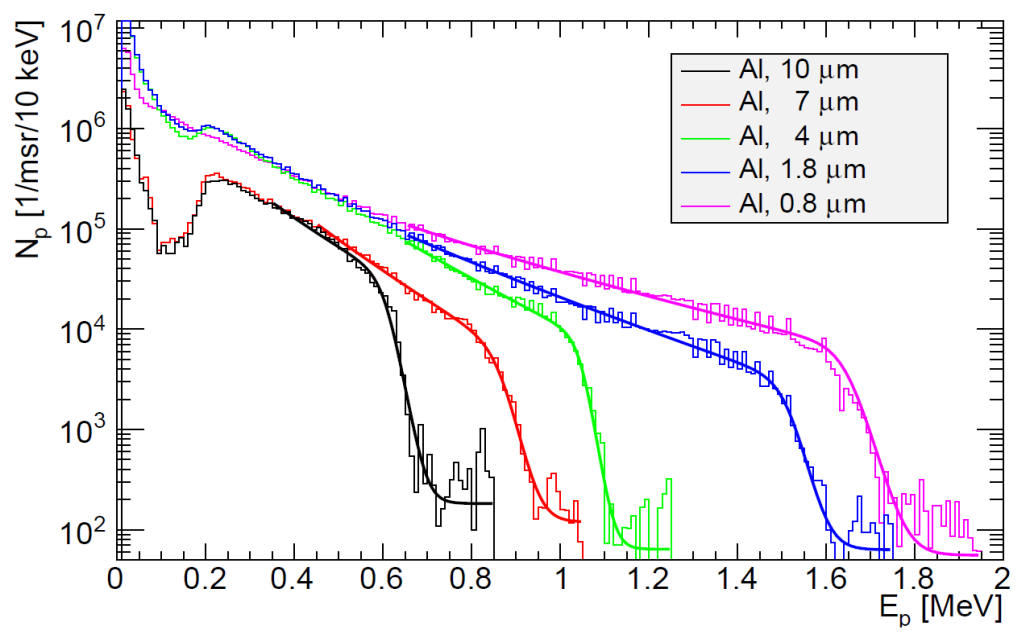

(b)

Figure 6.12: Experimental results from the low contrast experimental performed with $195 \mathrm{~mJ}, 50 \mathrm{fs}$ pulses and an intensity of $1.8 \cdot 10^{18} \mathrm{~W} / \mathrm{cm}^{2}$. (a) Image of etched CR-39 chips for several targets: aluminium from 4 to $18 \mu \mathrm{m}$ thickness and 6 and $13 \mu \mathrm{m}$ Mylar. (b) Spectrum of the protons accelerated in the low contrast regime extracted from CR-39 tracks for aluminium targets of different thickness. 


\subsection{Discussion}

In the two campaigns of experiments, apart from the increase in laser energy, the contrast of the laser was enhanced. Significant variations of the results were found between the two settings. The most relevant was the lack of accelerated protons with the mylar and thinner aluminium targets in the first campaign, which urged an explanation. The two possible explanations are that in the first case the energy of the laser was not enough to trigger efficient acceleration in those targets, or that the contrast was hindering the absorbed laser energy. In order to test these hypotheses, a new series of experiments were performed in the high energy configuration, though purposely deteriorating the contrast by removing one of the saturable absorbers. The outcome improved slightly the results of the first experimental campaign as the aluminium targets provided proton energies close to $1 \mathrm{MeV}$ but were not anywhere near the results of the high contrast campaign; therefore it was concluded that for the acceleration efficiency, contrast was more important than the energy. Similar conclusions have been reached by other authors [76,77] with experimental data and PIC simulations. They argue that when the pedestal is sufficiently intense it may arrive at the surface of the foil and heat the whole target, sending a shock wave that propagates through the material and results in the expansion of the target and particularly the rear surface, which deteriorates the acceleration mechanism when the main pulse reaches the target, as discussed in section 6.1.

A comparison of the maximum energy of protons accelerated during the experimental campaigns with other experiments found in the literature can be viewed in figure 6.13. The data has been limited to femtosecond lasers with less than 1 $\mathrm{J}$ of energy per pulse and acceleration in solid targets, and classified in function of the contrast of the laser; low contrast was considered as a ratio lower than $10^{7}$ between the main pulse and the pedestal [72,78-81] and high contrast as higher than $10^{7}$ [22, 40,77,82-87]. Two trends can be discerned, as high contrast experiments often result in more energetic protons and thinner materials. Daido et al. [20] suggest a simple empirical scaling law of maximum proton energy proportional to the square root of the laser pulse energy:

$$
E_{\text {max }}=k \sqrt{E_{\text {laser }}}
$$




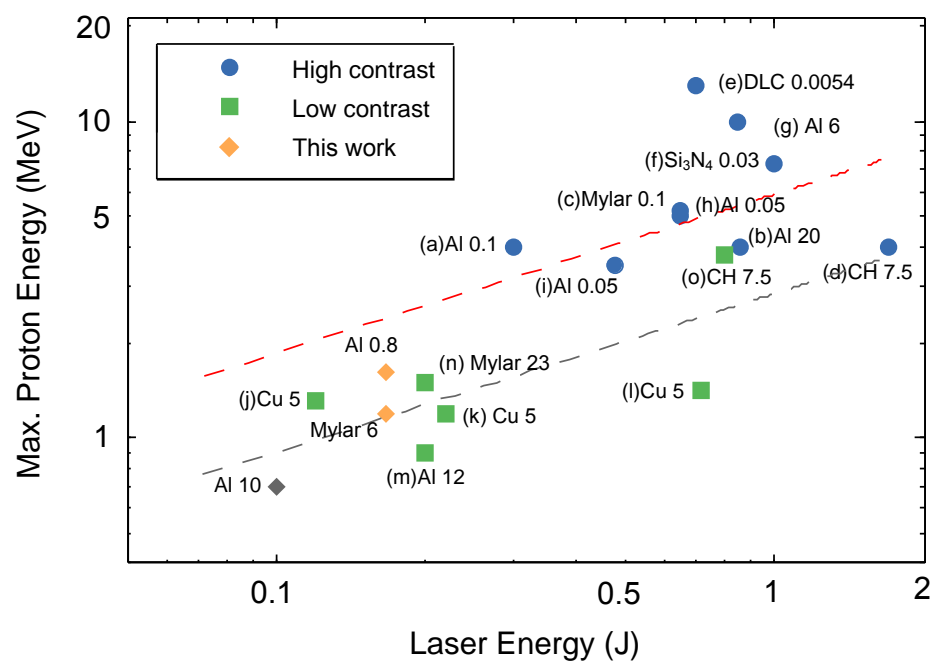

Figure 6.13: Experimental results of proton acceleration experiments found in literature. Blue circle dots are results for high contrast lasers while green square points are low contrast experiments. Diamond-shaped points represent the results obtained in this thesis. Each label displays the reference and the material and thickness (in microns) of the foil used in the experiment. (a) Neely et al. [82], (b) Kaluza et al. [40], (c) Ceccotti et al. [77], (d) Nishiuchi et al. [83], (e) Henig et al. [84],(f) Antici et al. [85], (g) Fritzler et al. [22], (h) A.P Robinson et al. [86], (i) J.S. Green et al. [87], (k) Fuiji et al. [81], (l) and (o) Yogo et al. [72,80], (m) and (n) Spencer et al. [79]. The dashed lines represent trend lines described by eq. 6.8 for $\mathrm{k}=3$ (low contrast) and $\mathrm{k}=6$ (high contrast).

A plot of these trend lines can be seen in fig. 6.13. A line with $\mathrm{k}=3 \mathrm{MeV} \mathrm{J}^{-1 / 2}$ (grey dashed line) seems to fit reasonably well the low contrast data, while a line with $\mathrm{k}=6 \mathrm{MeV} \mathrm{J}^{-1 / 2}$ works for high contrast data (red dashed line). Our result of proton acceleration with a foil of $10 \mu \mathrm{m}$ during the low contrast experiments is consistent with the grey line while the results with Mylar in the high contrast regime are also compatible with this trend; the aluminium data with a $0.8 \mu \mathrm{m}$ foil can be matched with the low or high contrast lines. These results support the evidence that the contrast ratio of the laser during the high contrast acceleration campaigns was close to $10^{7}$.

However, this scaling law does not take into account the properties of the target material, the width of the focal spot or the pulse duration of the laser. Another scaling law was introduced by Zeit et al. [88] that includes a dependence in all 


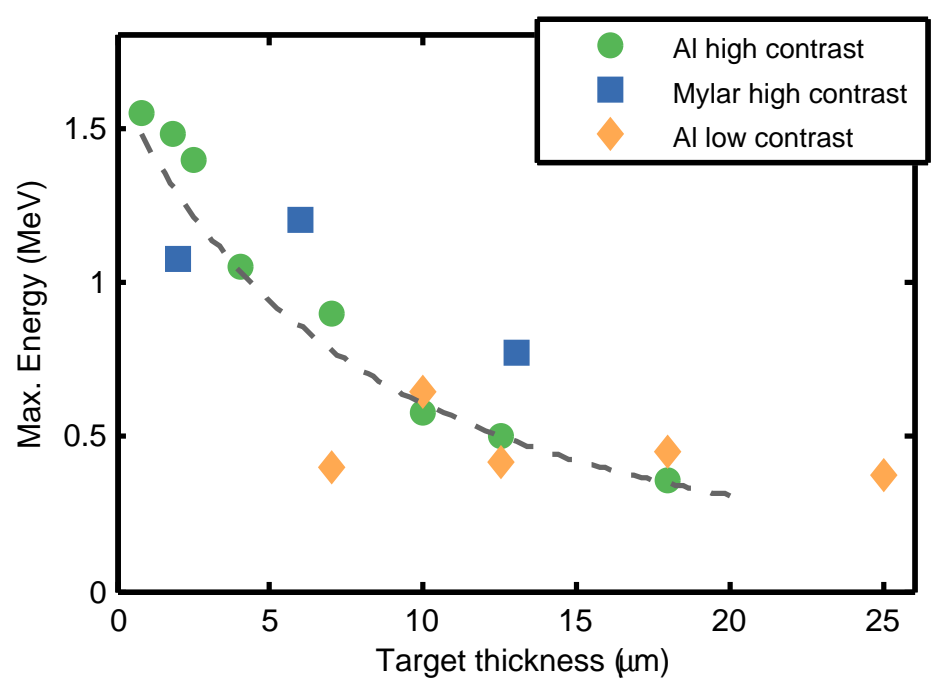

Figure 6.14: Maximum proton energy of laser accelerated protons with respect to the thickness of the target foil. Orange diamond-shaped points represent data acquired during the low contrast campaigns for aluminium foils. Blue squares and green circles are high contrast data for Mylar and aluminium foils respectively. The grey dashed line is the result of applying eq. 6.9.

these parameters:

$$
E_{\text {max }}=2 m_{e} c^{2} \sqrt{\frac{\eta P_{\text {laser }}}{P_{0}}} \tanh \left(\frac{\tau_{\text {laser }}}{2 \tau_{0}}\right)
$$

where $m_{e}$ is the mass of the electron, $\eta$ is the conversion efficiency into hot electrons, $P_{\text {laser }}$ is the peak power of the laser, $P_{0}$ is a the relativistic power unit $(8.7 \mathrm{GW})$, $\tau_{\text {laser }}$ is the pulse duration of the laser and $\tau_{0}$ is a reference pulse time that depends on the foil thickness, the focal spot of the laser and the divergence of the protons. Eq. 6.8 can be recovered if $\tau_{\text {laser }} \gg 2 \tau_{0}$.

These studies also predict the existence of an optimum foil thickness that, for a given material, depends strongly on the contrast of the laser. Figure 6.14 shows the dependence of the proton maximum energy on target thickness in this experiment; in the low contrast experiments, the maximum proton energy is reached with a $10 \mu \mathrm{m}$ target but no acceleration was observed for targets of $4 \mu \mathrm{m}$ or less, while for high contrast the optimum energy seems to peak close to the $0.8 \mu \mathrm{m}$ thickness target. In the case of the Mylar targets, the experiments performed in the low contrast regime resulted in no accelerated protons while in the high contrast regime, 
a maximum energy of $1.2 \mathrm{MeV}$ was reached for a thickness of $6 \mu \mathrm{m}$. The trend line obeys equation 6.9 with the following parameters: 4 TW peak power, 0.2 conversion efficiency, $6 \mu \mathrm{m}$ focal spot diameter and $10^{\circ}$ proton divergence, which fit remarkably well the experimental data of the aluminium foils but not that well in the case of the Mylar films.

The comparison of our results with other experiments in scientific literature demonstrate that the proton energies observed in our experiments are consistent with theoretical scaling laws and previous empirical results. 


\section{Conclusions}

In this thesis, we have detailed the development of a TW-level, high average power Ti:Sa laser for proton acceleration. The motivation was the need for high average power ultrashort systems, since the state-of-the-art lasers are capable of reaching record high intensities $\left(2 \cdot 10^{22} \mathrm{~W} / \mathrm{cm}^{2}\right)$ but average power below $100 \mathrm{~W}$. Most applications require both high average and peak power, such as the generation of diagnostic radioisotopes, which we identified as one of the most promising applications short-term for high power lasers.

The choice of Ti:Sa as the active medium responded to several criteria. First, because of the maturity of the technology associated with this material, as there are many systems all over the world and are covered extensively in the literature. Second, because of mechanical and thermal properties of the material. It is very resistant mechanically and has high thermal conductivity which would help for the thermal loads involved in a high average power laser. Third, because its wide spectral bandwidth allows for very short pulses, in the order of femtoseconds and for very high peak intensity, at moderate energies. However, the input energy for this material has to be provided optically in the visible region of the spectrum and in very short times. This required the development of high power lasers for pumping the Ti:Sa, because of the unavailability of commercial lasers of this kind.

One of the bottlenecks in the construction of a Ti:Sa laser is the unavailability of high power pump sources whose wavelength falls within the absorption cross section of Ti:Sa. Among high power lasers in the visible region of the spectrum, the most common lasers are based on Nd ions, and particularly on Nd:YAG and Nd:YLF. Therefore, we had to develop, in collaboration with the company Monocrom, a diode-powered pump head, the PH5400, since the use of flashlamps limits the achievable repetition rate. The simulation of this device is important for the 
optimization of its design, hence a Montecarlo ray-tracing code was developed for this purpose. It was developed in Matlab and when compared with experimental data was found to be in good agreement. The next step was to test the performance of the pumping heads in the laboratory. Tests were done for Nd:YAG and Nd:YLF and it was found that $\mathrm{Nd}$ :YAG could work at $1 \mathrm{kHz}$ repetition rate while maintaining a constant energy while Nd:YLF had to be limited to $200 \mathrm{~Hz}$ in order to prevent the fracture of the crystal. During these tests, the most important parameters related to the PH5400 pumping head could be characterized, such as the gain and slope efficiency.

Since a laser for pumping a Ti:Sa must have pulse duration less than microsecond, it was necessary to implement a Q-switching system for the pumping heads. It was found that a Q-switched pumping head with a Nd:YAG active medium could provide about $100 \mathrm{~mJ}$ per pulse and a Nd:YLF, $250 \mathrm{~mJ}$ per pulse, with pulse durations ranging from 10 to $20 \mathrm{~ns}$ at full power. This was not enough to pump the Ti:Sa laser, hence, in order to achieve higher energies, a MOPA laser was constructed with both materials that included up to eight fully syncronized pumping heads. The one with Nd:YAG was capable of delivering $400 \mathrm{~mJ} @ 532 \mathrm{~nm}$ and was comprised of eight pumping heads. The one with Nd:YLF could deliver $500 \mathrm{~mJ} @ 527 \mathrm{~nm}$ with five pumping heads. In order to circumvent size-related restrictions, each laser had two output beams, and in order to improve the overall efficiency of the second harmonic conversion, we used two KTP crystals, and the non-converted fundamental harmonic of the first KTP was further amplified to be converted into second harmonic radiation in a second KTP.

These MOPA systems proved to be scalable, since the only limitation to the achievable energy was the diameter of the rods. In fact, the pumping heads loaded with Nd:YLF operated at nearly half of their potential. Our simulations showed that the Nd:YLF MOPA could provide a total energy of $1 \mathrm{~J} @ 527 \mathrm{~nm}$ (500 mJ per beam). Recent experiments not covered in this thesis confirmed these estimations.

The Ti:Sa laser was constructed in a CPA configuration. It comprised an oscillator, a pulse stretcher, a regenerative amplifier, two multipass amplifiers and a pulse compressor. The oscillator was a commercial purchase (from Venteon) but all the other components were designed and built at Proton Laser Applications. The repetition rate of $100 \mathrm{~Hz}$ was possible through a combination of diode-pumped lasers 
and methods for alleviating the thermal load thorough all amplifiers. It is important to note that we did not use any cryogenic cooling and the compensation of thermal distortions was made passively via optics, which contributed to the simplicity and compactness of this TW laser. The lowest pulse duration achieved was 42 fs and the maximum energy of the Ti:Sa laser after compression was $315 \mathrm{~mJ}$. The contrast of the laser could not be fully characterized with a third-order autocorrelator, and hence we only got an estimate measurement with a fast photodiode.

Once the Ti:Sa laser was operative, the next step was to accelerate protons. These experiments were single-shot and did not use the full repetition rate of the laser system, as the development of targetry able to work at $100 \mathrm{~Hz}$ in vacuum is not trivial and out of the scope of this thesis, since it involves a remarkable engineering effort. The targets used in these experimental campaigns were thin foils of metal (Al) and plastic (Mylar) of different thicknesses. The experiments provided a maximum proton energy close to $2 \mathrm{MeV}$ for $4 \mathrm{TW}$ of laser power.

The energy of the accelerated protons in the experiments performed were below the threshold for the nuclear reactions that generate the radioisotopes (see figure 1.2), hence the main objective of this thesis could not be completely finished. However, to our knowledge, this was the first time that a laser succeeded in the accelerated of protons with energy higher than MeV in Spain.

In future developments, the energy per pulse of the laser must be improved since the ultimate goal of the project, the generation of clinical doses of radioisotopes, cannot be achieved with the current state of the system, because the proton energy is below the threshold to trigger the nuclear reactions. In parallel, further research is required in the area of targetry. Novel microstructured targets offer the possibility of improving both the maximum energy of the protons and efficiency of acceleration. A recent comparison of data, including experiments on ultra-thin foils as well as low-density and microstructured targets, suggests a steep dependence of the maximum proton energy with the energy of the laser. Therefore, a small increase in laser energy could represent a large step towards the requested proton energies for clinical doses of radioisotope production. The simulation tools developed in this thesis, allow to conclude that this laser energy increase could be achieved simply, with the same setup, by replacing the $5 \mathrm{~mm}$ diameter Nd:YLF rods with $6 \mathrm{~mm}$ ones. 


\section{Spanish summary / Resumen en español}

\section{"Diseño y desarrollo de un láser de alta potencia y alta tasa de repetición para la aceleración de protones"}

Esta tesis está estructurada en seis capítulos. El primero de ellos trata de ser una introducción a la tecnología láser y exponer la motivación que llevó a la realización de este trabajo.

En el segundo capítulo se estudia brevemente el concepto de amplificación y el modelo teórico de Frantz-Nodvik para la amplificación. En él también se tratarán las limitaciones y problemas asociados a la amplificación de pulsos cortos.

El tercer capítulo introduce uno de los dispositivos fundamentales para la realización del trabajo de esta tesis: el cabezal PH5400 de Monocrom bombeado por diodos. En el mismo capítulo se realiza una completa caracterización del dispositivo en modo pulsado quasi-continuo además de exponer algunos de los problemas asociados a su funcionamiento a alta potencia media.

El cuarto capítulo cubre la construcción de los láseres de bombeo de nanosegundo. Un solo cabezal PH5400 no es capaz de proporcionar gran potencia, pero el uso de varios en cadenas de amplificadores puede generar un láser de nanosegundo de gran potencia. Este capítulo abarca el desarrollo de dos láseres de alta potencia media: el primero usando Nd:YAG y el segundo Nd:YLF.

En el quinto capítulo se detalla cada etapa de la construcción de un láser ultracorto y ultraintenso. Se utilizará el Titanio:Zafiro como medio activo y los láseres desarrollados en el cuarto capítulo como bombeos. El láser desarrollado utiliza la técnica de "Chirped Pulse Amplification" (CPA) para obtener un pulso 
ultracorto de alta energía.

Por último, en el sexto capítulo se abordan los experimentos de aceleración de protones. En primer lugar, se describe el montaje experimental así como los tipos de detectores nucleares utilizados. A continuación se exponen las características más importantes de las partículas aceleradas en dos regímenes distintos y se comparan los resultados de este experimento con la literatura científica.

\section{Capítulo 1. Introducción y motivación}

Desde el nacimiento del láser en la década de los 60, no ha parado de crecer las aplicaciones y usos de estos dispositivos. Según han avanzado las técnicas se han conseguido llegar a generar pulsos de luz de nanosegundos $\left(10^{-9}\right.$ segundos $)$ mediante Q-switch, picosegundos (10 ${ }^{-12}$ segundos) e incluso femtosegundos $\left(10^{-15}\right.$ segundos) usando mode-locking. Durante las últimas décadas, los avances tecnológicos en láseres de alta potencia han avanzado a pasos agigantados y ya es común el uso de láseres de petawatio $\left(\mathrm{PW}=10^{16} \mathrm{~W}\right)$ en muchos centros de investigación en el mundo.

Uno de estos láseres ultraintensos puede ser focalizado a tamaños próximos a unas pocas micras que equivale a intensidades del orden de $10^{18} \mathrm{~W} / \mathrm{cm}^{2}$. A estas intensidades, el campo electromagnético del láser induce fuertes efectos no lineales sobre la dinámica de los electrones. Los electrones adquieren tal cantidad de energía que pueden llegar La aceleración de partículas por medio de láser es una de las aplicaciones más prometedoras debido al gran gradiente de aceleración que puede imprimir, del orden de $\mathrm{MeV}$ por micra, comparado con gradientes del orden de $\mathrm{MeV}$ por metro que son capaces de alcanzar los aceleradores convencionales de radiofrecuencia [8]. Sin embargo, los haces de partículas acelerados por láser no mejoran todas las características de los aceleradores de radiofrecuencia, como en control espectral, los haces generados por láser tienen un espectro ancho, o la corriente. La falta de corriente se debe a la falta de potencia media en los láseres actuales capaces de realizar dicha tarea.

Una de las aplicaciones al alcance a corto plazo de la tecnología láser es la aceleración de partículas para su uso en diagnóstico médico PET. PET (Tomografía por Emisión de Positrones) es una técnica de imagen que se basa en la recepción 
de pares de fotones gamma procedentes de la aniquilación electrón-positrón dentro del cuerpo del paciente. Los positrones son generados por decaimiento beta de un radiofármaco, una molécula orgánica a la que se ha añadido un isótopo inestable. La misión del radiofármaco es viajar mediante procesos fisiológicos a la zona del cuerpo con interés de estudio para localizar la emisión de positrones y esa zona pueda ser detectada para realizar una imagen médica.

Los principales isótopos emisores de positrones usados son ${ }^{11} \mathrm{C}^{13} \mathrm{~N}^{15} \mathrm{O}$ y ${ }^{18} \mathrm{~F}$. De entre todos ellos, ${ }^{18} \mathrm{~F}$ es el más utilizado sin duda debido a su relativamente larga vida media y su capacidad de unirse fácilmente a una molécula de glucosa, en particular el FDG, 2-fluoro-2-desoxiglucosa. Este radiofármaco es asimilado rápidamente y viaja en las zonas de alto consumo metabólico, como los tumores. Combinado con una técnica como una tomografía axial computerizada (TAC) o resonancia magnética (RMN) permite la localización precisa de una tumoración.

Uno de los mayores obstáculos para la difusión de la tecnología PET es la producción y logística de los radioisótopos. Actualmente los radioisótopos son generados exclusivamente en ciclotrones; estos aceleradores son costosos y requieren un gran espacio de alojamiento por lo que no suelen encontrarse en los propios hospitales o clínicas sino. Esto implica que uno de estos ciclotrones ha abastecer en una misma región a varios hospitales, algunos de los cuales pueden estar a varios cientos de kilómetros de distancia, lo que deriva en una distribución extremadamente costosa del radioisótopo. Este problema podría ser solucionado sustituyendo el ciclotrón por un acelerador láser; un láser ocupa unas dimensiones mucho más modestas que un acelerador de radiofrecuencia y solo necesitaría protección radiológica en la cámara de interacción entre el láser y el blanco. De esta manera se podría instalar un láser dentro del propio hospital y mejorar de manera exponencial el diagnóstico PET.

Varias pruebas de concepto se han realizado al generar pequeñas dosis de fluor-18 al bombardear un blanco de agua pesada con protones acelerados por láser [21-23]. Sin embargo, la consecución de una dosis humana de radioisótopo no puede alcanzarse con el estado del arte de los láseres ultraintensos. Para esta tarea es necesaria la consecución de un láser de alta potencia de decenas de teravatios $\left(\mathrm{TW}=10^{12} \mathrm{~W}\right)$ pero con una potencia media de unos $500 \mathrm{~W}[24,25]$.

El principal objetivo de esta tesis es realizar una contribución al desarrollo de 
láseres de alta potencia pico y alta potencia media con el objetivo futuro de alcanzar los parámetros requeridos para la generación de una dosis clínica de radiofármaco. Se utiliza el Titanio:Zafiro (Ti:Sa) como medio para desarrollar el láser debido a la madurez tecnológica asociada y a su capacidad de generar pulsos ultracortos de manera fiable. Los láseres actuales de Ti:Sa no son capaces de alcanzar una alta potencia media debido a la falta de un bombeo potente. Durante esta tesis se creó un bombeo de estado sólido basado en diodos, mucho más eficiente que uno convencional con el que se pudo alcanzar una frecuencia de repetición 10 veces mayor que los bombeos actuales $(100 \mathrm{~Hz}$ respecto a $10 \mathrm{~Hz}$ ).

\section{Capítulo 2. Amplificación de pulsos}

La generación y amplificación de pulsos cortos es uno de los temas más activos en la tecnología láser. Continuamente se están generando nuevos materiales con el objetivo de encontrar los parámetros óptimos para su funcionamiento como láser.

La amplificación, tanto como la generación de un haz láser, requiere de un medio con inversión de población y la presencia de emisión estimulada. Su dinámica depende del esquema de niveles electrónicos del propio medio activo: un sistema de dos niveles no puede generar inversión de población, mientras que uno de tres o cuatro niveles sí. En esta tesis utilizaremos Nd:YAG, Nd:YLF y Ti:Sa, que son buenos representantes de un sistema de cuatro niveles. Este esquema requiere que el bombeo se realice entre dos niveles, que denominaremos 1 y 4, mientras que la transición láser ocurre entre los niveles 2 y 3 (mayor numeración del nivel, mayor energía). De esta manera, el fotón de bombeo absorbido hace que el electrón pase al nivel 4, que decae rápidamente al nivel 3 de manera no radiativa. Es el nivel 3 metaestable el que decae al 2 emitiendo un fotón de longitud de onda mayor que el bombeo; en el nivel 2 el electrón nuevamente decae de manera no radiativa al nivel fundamental. La rapidez de las transiciones no radiativas son las que hace que en un esquema de cuatro niveles se pueda mantener una abundante inversión de población.

Para describir teóricamente la amplificación se parte de las ecuaciones de balance, un sistema de ecuaciones diferenciales acopladas que describen la evolución del flujo y la inversión de población en un láser. 
Si suponemos que el medio activo ya ha sido bombeado, que no hay pérdidas y que la amplificación se produce de manera instantánea, podemos realizar una integración en tiempo de las ecuaciones anteriores y operar hasta llegar a la conocida como ecuación de Frantz-Nodvik [29]:

$$
F(z)=F_{\text {sat }} \ln \left(1+\exp (g z)\left(\exp \left(\frac{F(0)}{F_{\text {sat }}}\right)-1\right)\right)
$$

La fluencia $F$ se define como la energía por unidad de superficie:

$$
F(z)=\int_{-\infty}^{\infty} I(z, t) d t
$$

La ecuación R.1 relaciona la fluencia a la salida del amplificador con la fluencia de entrada a través de la fluencia almacenada en el medio activo $F_{\text {stor }}$ que depende del bombeo. La fluencia de saturación $F_{\text {sat }}$ es un parámetro que solo depende del medio activo y la longitud de onda:

$$
F_{\text {sat }}=\frac{h \nu}{\sigma}
$$

De esta ecuación se puede deducir mucha dinámica interesante de la amplificación. Si la fluencia de la semilla que va a ser amplificada es baja entonces podemos hacer la aproximación $\exp \left(F(0) / F_{\text {sat }}\right) \approx 1+F(0) / F_{\text {sat }}$ y la ecuación R.1 se convierte en:

$$
F(L)=F_{0} \exp (g L)
$$

Este régimen de amplificación se denomina régimen lineal debido a que la fluencia de salida y de entrada se relacionan mediante una constante de proporcionalidad que denominaremos ganancia y que depende de la energía almacenada en el medio activo:

En cambio, si la energía de la semilla es elevada entonces podemos aproximar $\exp \left(F(0) / F_{\text {sat }}\right) \gg 1$ y la ecuación R.1 se convierte en:

$$
F(L)=F_{\text {sat }}\left(g L+\frac{F(0)}{F_{\text {sat }}}\right)=F(0)+F_{\text {sat }} g L
$$

Este régimen se denomina de saturación. La fluencia extraída tan solo depende 
de parámetros propios del material y de la energía almacenada, que se corresponde con el máximo que se puede extraer del amplificador.

Nótese que la ganancia, definida como el cociente entre la fluencia de salida y la de entrada es próxima a la unidad en el régimen de saturación aunque la eficiencia de extracción de energía es máxima, contrario a lo que ocurre en el régimen lineal. Por tanto, no es posible obtener a la vez alta ganancia y alta extracción de energía, sino que deberemos buscar en muchas ocasiones un balance entre ambas.

Además, el proceso de amplificación puede estar limitado por condiciones externas, la mayoría relacionadas con daño óptico en los materiales. Si la fluencia supera un valor umbral, entre $10 \mathrm{~J} / \mathrm{cm}^{2}$ para una superficie con coating y hasta $100 \mathrm{~J} / \mathrm{cm}^{2}$ para una superficie pulida, se producirá daño producido por el propio láser sobre dicha superficie.

A pesar de que la ecuación de Frantz-Nodvik solo se utilizó un formalismo unidimensional, ésta se puede ampliar sin pérdida de generalidad para incluir las coordenadas transversales $x$ e $y$ de tal manera que la amplificación pasaría a describirse como un problema local sobre cada punto de la superficie independiente del resto. Esto implica que la amplificación dependerá tanto del perfil espacial de la semilla como de la distribución de energía almacenada en el medio activo, lo que lleva a situaciones en las que el perfil espacial de salida pueda ser deformado.

Una situación parecida ocurre si se expresa la ecuación de Frantz-Nodvik en función del tiempo, debido a que la parte del pulso que llega primero al medio activo experimenta más ganancia que la parte trasera. En un láser CPA la frecuencia instantánea equivale a un intervalo temporal por lo que también pueden sufrir deformaciones del espectro, en concreto gain narrowing y gain shifting. Gain shifting produce un desplazamiento de la longitud de onda central del espectro mientras que gain narrowing produce un estrechamiento del espectro. En un pulso ultracorto la pérdida de ancho de banda produce un ensanchamiento temporal del pulso por lo que para obtener un pulso lo más corto posible se ha de intentar mitigar el efecto de gain narrowing.

Entre las deformaciones de la parte temporal que puede sufrir un pulso ultracorto se encuentra la inclusión de artefactos dentro de su estructura temporal. El contraste de un pulso mide el cociente entre la intensidad del pulso principal y la intensidad de cualquier punto situado a una cierta distancia. Un contraste malo puede ser capaz 
de afectar de manera negativa experimentos en los que se requiere la focalización del láser. La típica estructura temporal de un pulso puede observarse en la figura. Se observar la presencia de un pedestal que puede extenderse a cientos de picosegundos y que corresponde a ASE (emisión estimulada amplificada) el pedestal situado a femtosegundos puede ser producido por una mala compresión del pulso, mientras que los prepulsos y postpulsos suelen ser réplicas del pulso principal producidas por reflexiones en alguna superficie durante la amplificación.

Por último, otra de las limitaciones de la amplificación de pulsos ultracortos son los efectos no lineales. A pesar de haber sido estirados, los pulsos en un láser CPA pueden tener intensidad suficiente como para sufrir self-focusing en el medio amplificador. Al propagarse por el medio active, el pulso induce un cambio en el índice de refracción que crea una fase correspondiente a una lente convergente. Debido a que esa lente es proporcional a la intensidad, el pulso se focaliza cada vez más hasta convertirse en un haz minúsculo denominado filamento. Este efecto puede provocar daño ópticos en los cristales de amplificador, pero no solo afecta los láseres ultracortos; durante la realización de esta tesis se pudo observar la formación de filamentos que dañaron un cristal de Nd:YAG con pulsos de nanosegundo.

\section{Capítulo 3. Cabezales bombeados por diodos}

El bombeo por diodos láser se está convirtiendo cada vez en algo más común debido al desarrollo de dispositivos cada vez más potentes en la región del infrarrojo cercano.

Hasta ahora, la mayoría de los láseres de estado sólido eran bombeados por lámparas de flash. Este método permite depositar grandes cantidades de energía en el medio activo; sin embargo, el espectro de emisión de las lámparas de flash es ancho, siendo capaz de excitar otras transiciones electrónicas que contribuyen a generar calor dentro del medio activo. Además, en modo pulsado las lámparas de flash no suelen superar los $10 \mathrm{~Hz}$ de frecuencia de repetición y su vida útil es muy reducida. Esto hace que un bombeo por lámparas de flash sea ineficiente. En cambio un diodo láser, a pesar de no ser capaz de proporcionar tanta energía como una lámpara, puede alcanzar frecuencias de repetición mucho más altas en modo pulsado y su espectro de emisión es mucho más estrecho, de tal manera que puede 
ser ajustado para excitar únicamente la transición láser y generar mucho menos calor, por lo que el láser es mucho más eficiente. Para aprovechar las ventajas que proporcionan los diodos láser se ha de construir una cámara de bombeo que permita un acoplamiento eficiente de la luz de los diodos con el medio activo. Por ello desde Monocrom S.L se desarrolló un cabezal denominado PH5400 que permite el bombeo lateral de una barra cilíndrica de Nd:YAG o Nd:YLF.

El cabezal PH5400 está compuesto por 36 barras de diodos de $150 \mathrm{~W}$ de potencia óptica cada una, divididas en 6 stacks que cuentan con un sumidero de calor de cobre refrigerado por agua. Se les proporciona energía mediante pulsos eléctricos generados por una electrónica integrada en el propio cabezal. Los pulsos son cuadrados pudiendo recibir hasta 150 A de corriente pico. Dentro de la cámara de bombeo, el medio activo está rodeado simétricamente por los seis stacks de diodos. Para su refrigeración, el medio activo está sumergido en una capa de agua circulante confinada por un flowtube, un cilindro hueco de cuarzo transparente a la longitud de onda de los diodos.

La distribución de energía absorbida dentro del medio activo es un parámetro muy importante ya que rige la directamente la distribución de la ganancia, de la temperatura y del estrés mecánico. Por ello se realizaron simulaciones basadas en un código de Monte Carlo con trazado de rayos donde la luz de los diodos se trata como rayos que se propagan en una dirección aleatoria dentro del patrón de emisión de luz de los diodos láser.

Dentro del código, cada rayo sufre refracción tres veces hasta llegar al medio activo: en la superficie exterior del flowtube, en la interior que contacta con el agua y en la superficie exterior del medio activo donde el rayo es absorbido de acuerdo a la ley de Beer-Lambert. La superposición de una cantidad suficiente de rayos da lugar a una representación de la energía absorbida en el medio activo.

Para corroborar la bondad de las simulaciones, se realizaron medidas de la fluorescencia emitida por una barra de Nd:YAG de $5 \mathrm{~mm}$ de diámetro, otra de Nd:YAG de $6 \mathrm{~mm}$ de diámetro y una de Nd:YLF de $5 \mathrm{~mm}$ de diámetro. Uno de los resultados más llamativos aparece en la barra de Nd:YAG de $6 \mathrm{~mm}$ de diámetro, que puede observarse en la figura R.1. La parte central de la barra se encuentra menos bombeada y la energía absorbida está más acumulada en las zonas exteriores debido al dopaje de esta barra en concreto. La alta potencia media que es capaz de 


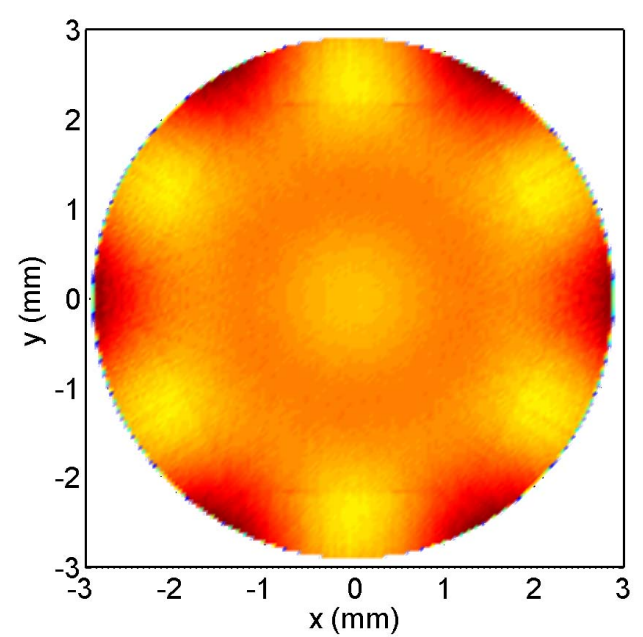

(a)

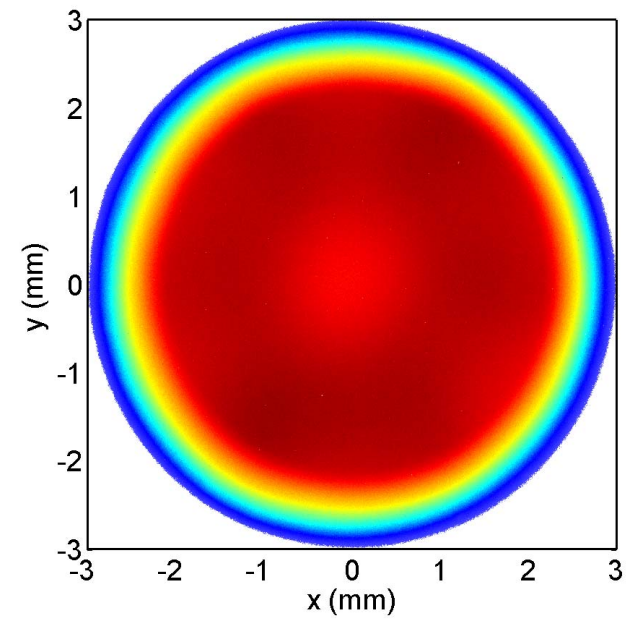

(b)

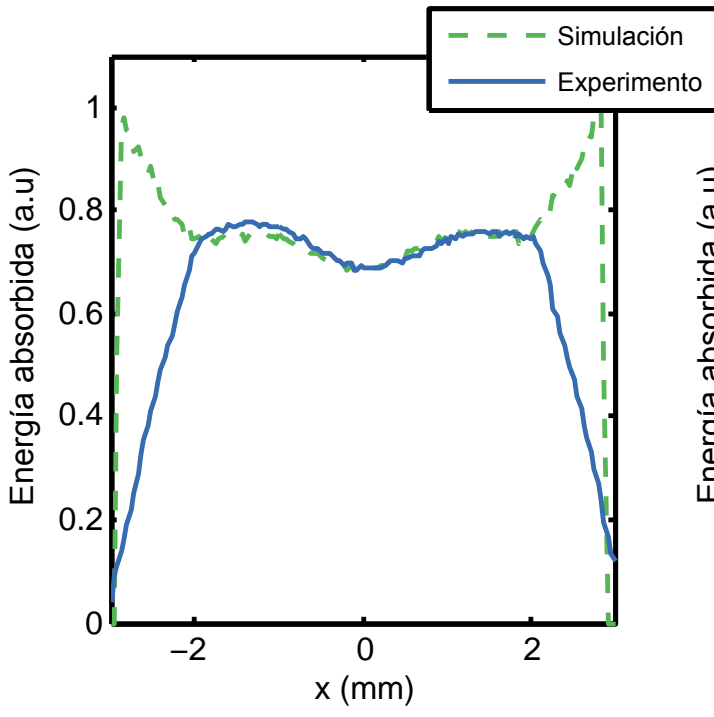

(c)

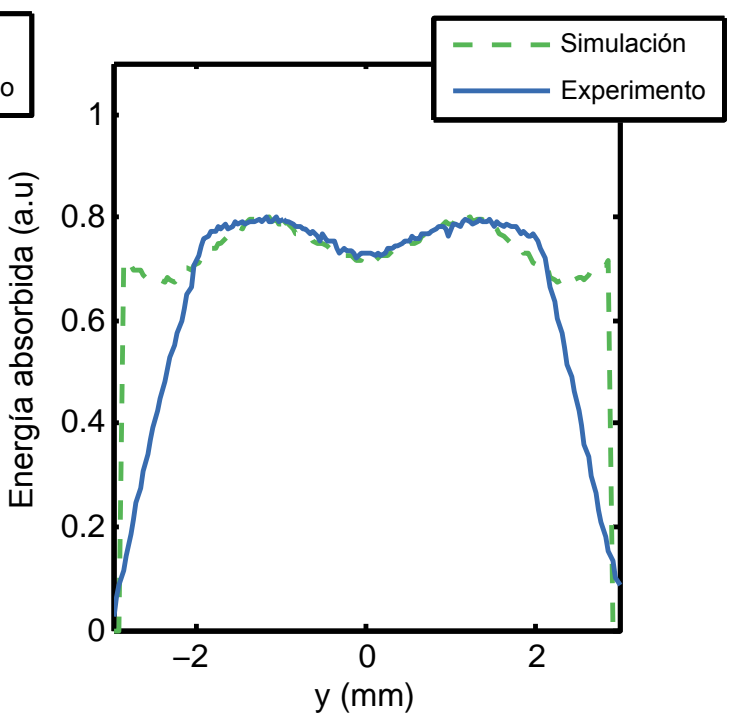

(d)

Figure R.1: Comparación de simulaciones y datos experimentales de la energía absorbida en una barra de Nd:YAG de $6 \mathrm{~mm}$ de diámetro y $0.6 \%$ at. de dopaje: (a) Simulación de la distribución de energía absorbida dentro de la barra de Nd:YAG. (b) Distribución de fluorescencia capturada con una cámara CCD. (c) Comparación de los cortes verticales de la simulación (línea discontinua) y de la imagen experimental (línea continua). (d) Comparación de los cortes horizontales de la simulación (línea discontinua) and experimental profile (línea continua). 
alcanzar un láser bombeado por diodos hace que aparezcan efectos térmicos debido al calentamiento del medio activo. Estos efectos, entre los que destacan la lente térmica y la birrefringencia inducida, disminuyen el rendimiento del láser pudiendo hasta evitar su funcionamiento.

El efecto de lente térmica se produce por cambios en el índice de refracción inducido por un gradiente de temperatura en el medio activo, lo que provoca que el propio láser actúe como una lente que suele ser convergente. La birrefringencia es causada por la fase introducida por dichos cambios en el índice de refracción sobre las componentes de la polarización de la luz (radial y azimutal en el caso de una barra cilíndrica) que entra al medio activo. Este efecto induce la depolarización del haz de luz. Ambos efectos deben ser mitigados en láseres de alta potencia, sobre todo en las etapas de amplificación.

Para comprobar el rendimiento de los cabezales PH5400 se montaron dentro de un resonador con únicamente dos espejos, el espejo trasero totalmente reflectante y el espejo de salida con una reflectancia de entre el 30 y $85 \%$. Los cabezales con Nd:YAG funcionaron en modo freerun o QCW (quasi-continuo), en el que la pulsación de los diodos de bombeo genera los pulsos de salida del láser. El bombeo consiste en pulsos de $200 \mu$ s de largo y 100 A de corriente pico. Con estos parámetros, el láser podía llegar a dar $200 \mathrm{~W}$ de potencia media a $1 \mathrm{kHz}$ de frecuencia de repetición, lo que equivale a $200 \mathrm{~mJ}$ por pulso. La energía por pulso se mantiene constante independientemente de la frecuencia de repetición elegida.

También se realizaron pruebas usando Nd:YLF. Este material puede lasear en dos longitudes de onda diferentes: $1047 \mathrm{~nm}$ o $1053 \mathrm{~nm}$ y tiene un tiempo de fluorescencia de $550 \mu \mathrm{s}$, casi tres veces mayor al de Nd:YAG, por lo que puede almacenar mucha más energía. De hecho con este material se llegó a alcanzar una potencia media de $65 \mathrm{~W}$ a $100 \mathrm{~Hz}$, lo que equivale a una energía por pulso de 650 mJ funcionando a la longitud de onda 1047 nm y un bombeo de $500 \mu$ s de duración y 100 A. Sin embargo, el Nd:YLF también es más frágil mecánicamente que el Nd:YAG y no se pudo superar $200 \mathrm{~Hz}$ de frecuencia de repetición debido al riesgo rotura de la barra. 


\section{Capítulo 4. Desarrollo de un láser de nanosegundo}

El buen rendimiento de los cabezales funcionando en modo quasi-continuo provocó que surgiera interés en comprobar su rendimiento usando alguna técnica de generación de pulsos cortos como Q-switch.

Para funcionar en Q-switch, los cabezales son introducidos en un resonador que contiene dos espejos, el trasero de reflectividad total y el espejo de salida, de reflectividad entre el 20 y 35 \%. Para realizar el Q-switch, se tiene una celda Pockels, una lámina cuarto de onda y un polarizador. La celda Pockels está hecha de $\mathrm{KD}^{*} \mathrm{P}$ y necesita $3.4 \mathrm{kV}$ para funcionar como lámina cuarto de onda.

Usando un PH5400 con Nd:YAG bombeado con pulsos de $200 \mu$ s y 100 A a $100 \mathrm{~Hz}$ se pudo alcanzar una energía por pulso de 105 mJ y 12 ns de duración. El modo espacial obtenido era muy multimodo, resultado de la excitación de muchos modos dentro del medio activo. Un modo gaussiano suele ser el preferido como perfil espacial del haz de salida de un oscilador, por lo que se buscó un método para suprimir el resto de modos. Dicho método consiste en la inserción de un telescopio dentro de la propia cavidad, de tal manera que aumenta el volumen ocupado por el haz fundamental dentro del resonador. Al realizarlo se pudo obtener un haz quasi-gaussiano de 10 mJ de energía y 16 ns de duración de pulso; sin embargo la energía no se pudo aumentar debido a que el telescopio hace más pequeño el haz de salida y puede superar el umbral de daño óptico.

Se realizó el mismo experimento con Nd:YLF dentro de los PH5400, bombeado con pulsos de hasta $500 \mu \mathrm{s}, 100 \mathrm{~A}$ y $100 \mathrm{~Hz}$. Se realizaron experimentos con ambas polarizaciones y se pudo alcanzar hasta $300 \mathrm{~mJ}$ de energía y 9 ns de duración de pulso a $1047 \mathrm{~nm}$. De nuevo el haz de salida era muy multimodo por lo que para mejorarlo se introdujo otro telescopio intracavidad. Los resultados fueron muy similares, llegando a obtener 60 mJ y 16 ns y una calidad de haz sobresaliente. Sin embargo, una de las mayores ventajas de Nd:YLF es su capacidad de almacenar energía por lo que este método es claramente subóptimo al estar limitada la energía por el tamaño del haz de salida.

Para aumentar la energía obtenida en modo Q-switch, se crearon dos láseres tanto para Nd:YAG como para Nd:YLF con arquitectura MOPA (Oscilador Maestro Amplificadores de Potencia), que consiste en un oscilador seguido de uno o varios 
amplificadores. El fin de estos MOPAs es bombear un cristal de Ti:Sa por lo que han de funcionar utilizando el segundo armónico.

Para el primer MOPA se utilizaron ocho cabezales PH5400 con Nd:YAG, dos funcionando dentro del oscilador y tres pares de amplificadores. La eficiencia es cercana al $65 \%$ y se llegaron a obtener hasta $240 \mathrm{~mJ}$ por cada rama con una longitud de onda de $532 \mathrm{~nm}$.

El segundo MOPA se realizó utilizando cinco cabezales PH5400 cargados con Nd:YLF, uno utilizado como oscilador y dos pares de amplificadores. Se estimó que se podría alcanzar $1 \mathrm{~J}$ de energía total en $527 \mathrm{~nm}$ si se sustituyeran las barras de los amplificadores de $5 \mathrm{~mm}$ de diámetro por otras de $7 \mathrm{~mm}$ de diámetro para disminuir el riesgo de daño óptico.

\section{Capítulo 5. Desarrollo de un láser ultraintenso}

Para la construcción del láser ultraintenso se recurrió a la arquitectura CPA usando un medio activo de Ti:Sa. La idea tras un láser CPA es la generación de un pulso ultracorto y energético; para ello se parte de un pulso ultracorto y de muy baja energía, se estira en tiempo para disminuir su intensidad y se amplifica. Una vez se ha conseguido un pulso estirado y de alta energía, el láser se hace pasar por un compresor que vuelve a estrechar temporalmente el pulso para acabar con un pulso ultracorto y de alta energía.

El esquema del láser CPA desarrollado durante esta tesis se encuentra en la figura R.2. La primera etapa es un oscilador de la casa comercial Venteon que proporciona pulsos de $5 \mathrm{~nJ}$, aproximadamente 12 fs y $80 \mathrm{MHz}$ de frecuencia de repetición.

El pulso proveniente del oscilador es estirado en tiempo en un estirador del tipo Offner, que consiste en una red de difracción, dos espejos curvos y un retroreflector; el láser incide sobre la red de difracción que envía el haz difractado hacia el par de espejos, uno convexo y el otro cóncavo, que se encargan de crear una imagen virtual de la red de difracción a una distancia negativa de esta. El haz cambia de altura gracias al retroreflector y se repite el proceso de tal manera que el láser ha realizado cuatro pasos por la red de difracción.

Una vez estirado el pulso se dirige al amplificador regenerativo pasando por 


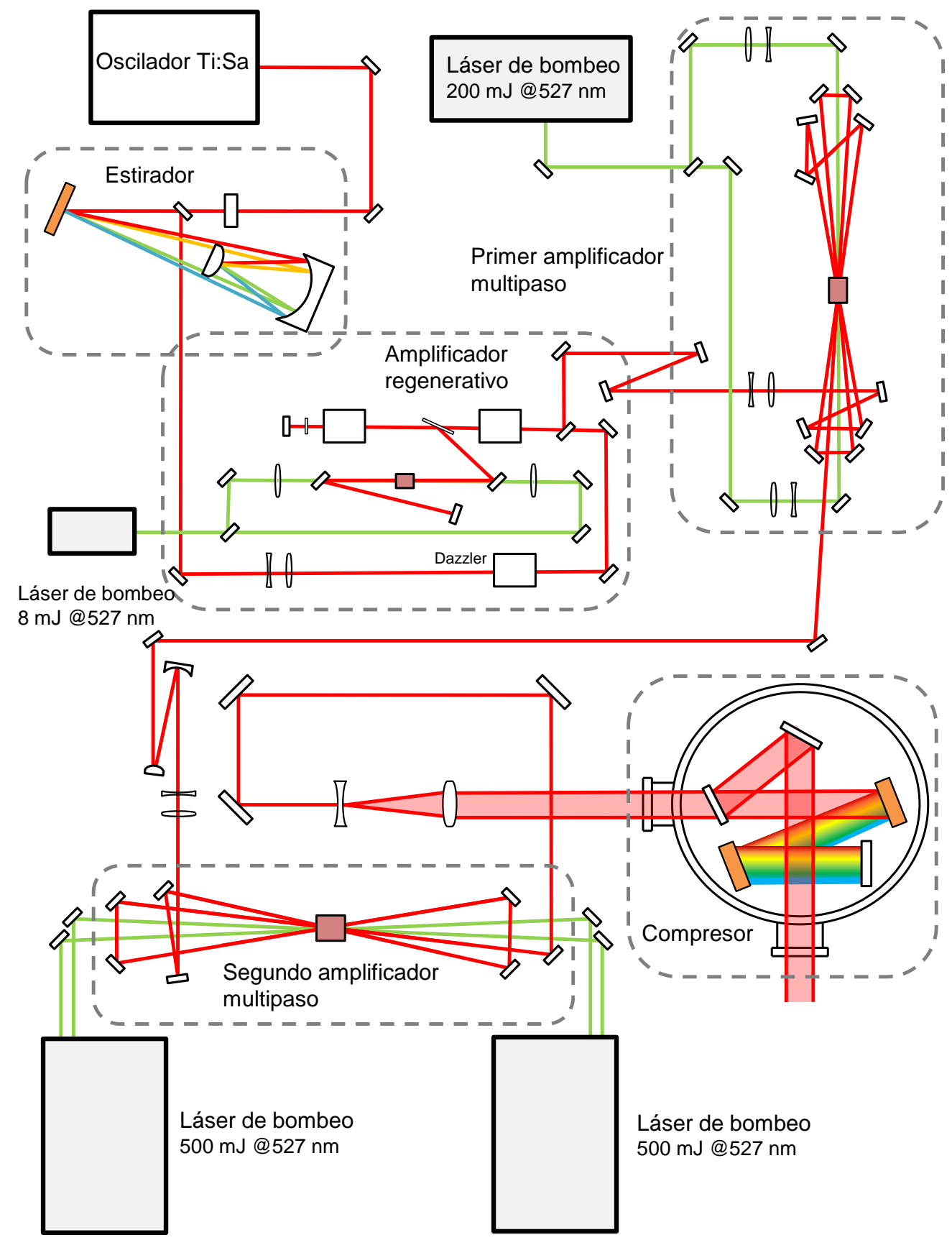

Figure R.2: Esquema del láser CPA ultraintenso desarrollado. Comienza en el oscilador de Ti:Sa, es estirado temporalmente en el estirador y comienza su amplificación en el amplificador regenerativo, sigue en el primer multipaso y termina en el segundo amplificador multipaso. A continuación el haz es agrandado para pasar al compresor, que se encuentra dentro de una cámara de vacío. La salida del compresor comunica con la cámara donde se realizarán los experimentos. 
el Dazzler, un dispositivo acusto-óptico con el que el usuario puede varia la fase introducida al pulso que sirve por ejemplo para cambiar de forma su espectro. Un amplificador regenerativo consiste en un resonador en el que la semilla entra a través de un polarizador y es atrapada dentro mediante la acción de una celda Pockels mientras se amplifica; una vez terminado este proceso la misma celda Pockels expulsa la semilla amplificada. En el amplificador regenerativo el pulso experimenta una ganancia de $10^{6}$ al salir con una energía por pulso de $1.5 \mathrm{~mJ}$ y haz gaussiano. Además, su frecuencia de repetición es reducida a 100 Hz.

El primer amplificador multipaso está construido en configuración lazo de tal manera que el haz atraviesa tres veces el medio activo, un cristal de Ti:Sa cilíndrico de $6 \mathrm{~mm}$ de diámetro. El cristal es bombeado por un pequeño láser MOPA de dos cabezales que proporciona $200 \mathrm{~mJ}$ en $527 \mathrm{~nm}$. La lente térmica del Ti:Sa empieza a ser problemática por lo que para mitigarla se sustituyeron algunos espejos planos del amplificador por espejos curvos convexos que introducen divergencia. El láser sale con una energía de $25 \mathrm{~mJ}$ por pulso y un haz de buena calidad.

Antes de entrar al segundo amplificador multipaso el haz atraviesa un par de espejos que aumentan su tamaño a $6 \mathrm{~mm}$ de diámetro, seguido de un telescopio de baja magnificación que sirve para ajustar la divergencia del haz y así compensar la lente térmica que sufrirá al amplificarse. Este amplificador multipaso también realiza tres pasadas por el medio activo pero consta de un cristal más grande de sección cuadrada de $15 \mathrm{~mm}$ de arista. Es bombeado por $1 \mathrm{~J}$ de energía dividido en cuatro haces de bombeo, cada uno de ellos de $250 \mathrm{~mJ}$ de energía provenientes de dos láseres MOPA de Nd:YLF descritos en el capítulo anterior. El láser es amplificado hasta los $320 \mathrm{~mJ}$ de energía por pulso.

Una vez amplificado el láser se dirige al compresor, que se encuentra dentro de una cámara de vacío. El compresor consta de dos redes de difracción y un retrorreflector que devuelve al haz a diferente altura para que incida sobre cada red de difracción dos veces. La medida de duración de pulso se realiza con el Wizzler, un dispositivo basado en SRSI [referencia] que puede comunicarse con el Dazzler para modificar la dispersión del láser y así optimizar la duración de pulso. Se midió una duración final de pulso compatible con 45 fs en un espectro de 19 nm; la duración del pulso límite de Fourier fue calculada como 42 fs.

Se pudo comprobar que el contraste del láser no era óptimo debido a que tras 


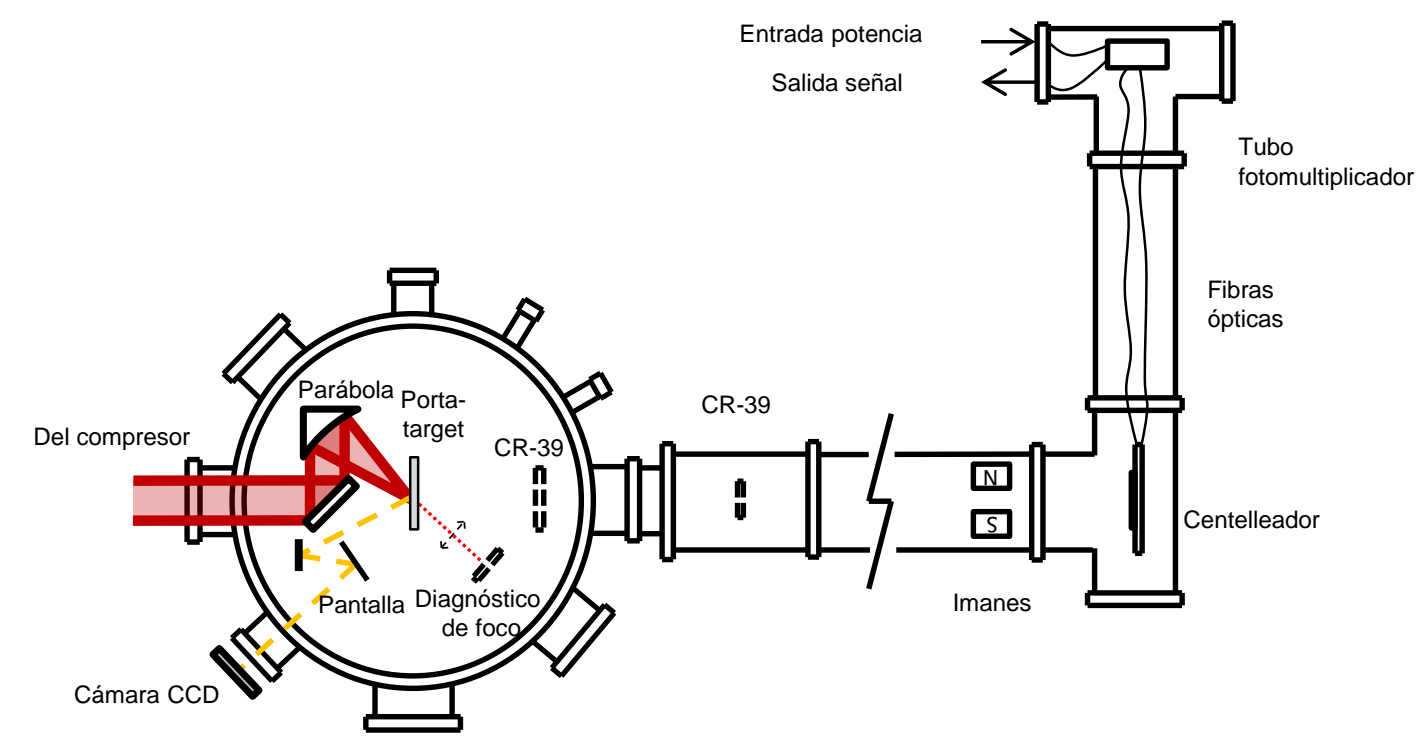

Figure R.3: Esquema del experimento de aceleración de protones. El láser es focalizado por una parábola fuera de eje sobre el target. Los chips de CR-39 se sitúan cerca y al final del tubo del tiempo de vuelo se encuentra el centelleador, cuya señal de salida se puede acoplar a un osciloscopio.

el regenerativo se observó la presencia de réplicas débiles del pulso que podía empeorar los resultados de los experimentos de aceleración. Por eso se introdujeron dos absorbentes saturables tras el regenerativo y tras el primer multipaso. Esto conllevó una pérdida de energía ya que el primer multipaso funcionaba con $25 \mathrm{~mJ}$ de salida y el segundo multipaso $265 \mathrm{~mJ}$.

\section{Capítulo 6. Experimentos de aceleración con láser}

Una vez completado el láser se realizaron experimentos de aceleración de partículas. Los experimentos se realizaron en modo single shot que consiste en enviar un único pulso del láser mediante un sistema de triggers electrónicos. No fue posible utilizar toda la frecuencia de repetición del láser por la dificultad técnica de un dispositivo que pueda refrescar un target a unos $100 \mathrm{~Hz}$ y sin que su posición sufra cambios en una escala de unas pocas decenas de micras.

Todo el instrumental utilizado en los experimentos de aceleración se encuentra situado en una cámara de vacío independiente de la del compresor, figura R.3. 
Como detectores de partículas se utilizaron varios instrumentos: chips de CR-39, unas láminas de resina especial que solo son sensibles a protones y otros iones pesados. Su gran ventaja es que la señal no está distorsionada por electrones ni el resto de radiación electromagnética emitida en la interacción del láser con el blanco, pero requiere de un proceso de relevado en una disolución básica a temperatura elevada por lo que hay que realizar una buena calibración previa. También se utiliza como detector un cristal centelleador que captura toda la radiación ionizante que le llega pero es capaz de mostrar la señal de protones en tiempo real. Para obtener la distribución espectral de los protones se utilizó el centelleador como detector en tiempo de vuelo. Este esquema requiere separar una distancia concreta ( $2 \mathrm{~m}$ en este experimento) la fuente de los protones y el detector de tal manera que los protones más energéticos tardan menos tiempo en llegar al centelleador que los protones de baja energía. De esta manera se recoge un espectro temporal que coincide con la distribución energética del haz de protones generado. Además se diseñó para este experimento una parábola de Thomson, un dispositivo con un campo magnético y eléctrico perpendiculares entre sí que permite desviar las partículas tanto por carga como por masa. Sin embargo, durante el experimento solo se utilizó el campo magnético para desviar los electrones mas energéticos y evitar que introdujeran ruido en la señal de protones.

El haz proveniente del compresor entra en la cámara de vacío y se focaliza mediante una parábola fuera de eje sobre un blanco, sólido en este experimento que puede ser una lámina muy fina (pocas micras de espesor) hecha de metal o plástico. El tamaño del foco se pudo medir atenuando el láser y utilizando una lente asférica para recoger la imagen en una cámara CCD. Se midió un foco de $10.5 \cdot 15.4 \mu \mathrm{m}$ medido a $1 / \mathrm{e}^{2}$ de la intensidad pico y se estimó que el $78 \%$ de la energía se encuentra recogida dentro de ese área. Para verificar que la superficie del blanco coincide con la posición del foco, se diseñó un sistema que recoge la señal de speckle generada por la superficie difusa del blanco al incidir sobre él un láser auxiliar de He-Ne alineado por el mismo camino óptico que el láser de TW.

Los experimentos se realizaron con dos condiciones del láser diferentes. En la primer tanda, cuyos resultados pueden verse en la figura R.4(a), el láser contaba con una energía por pulso de 97 mJ y 45 fs de duración de pulso mientras que la intensidad pico llegó al valor $1.2 \cdot 10^{18} \mathrm{~W} / \mathrm{cm}^{2}$ con un contraste de menor de $10^{5}$. 


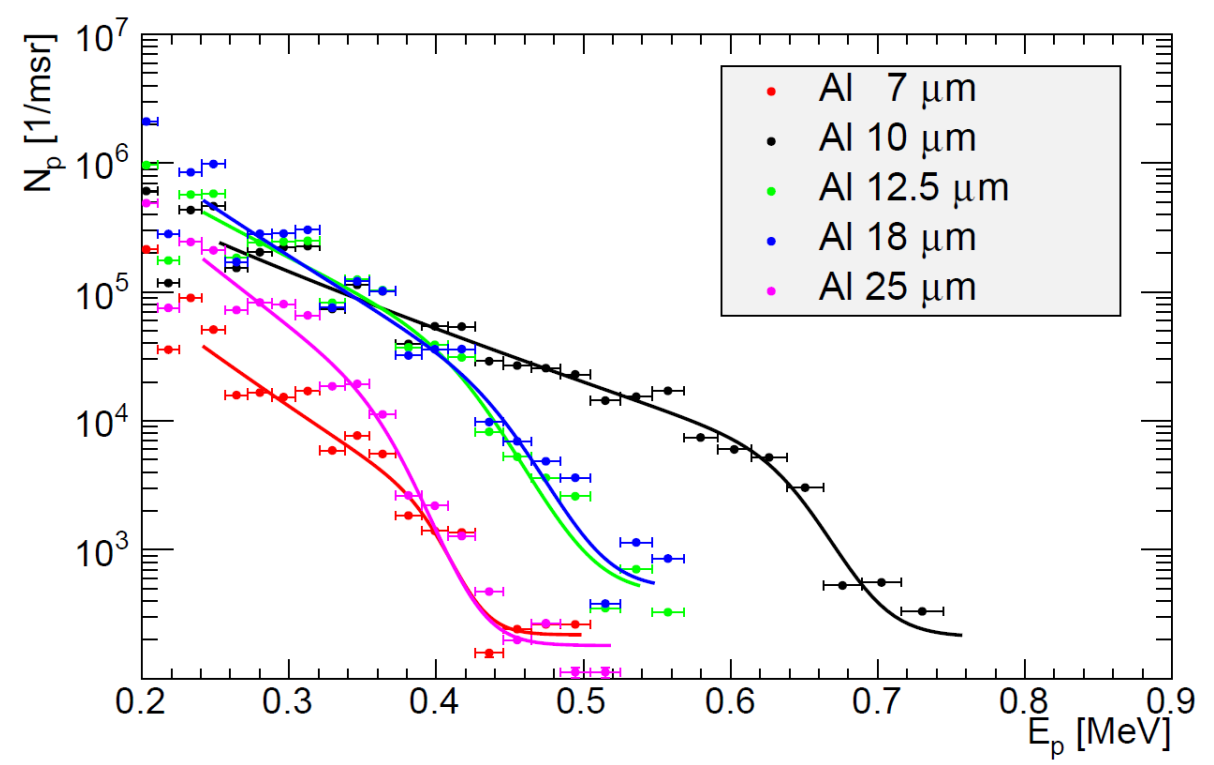

(a)

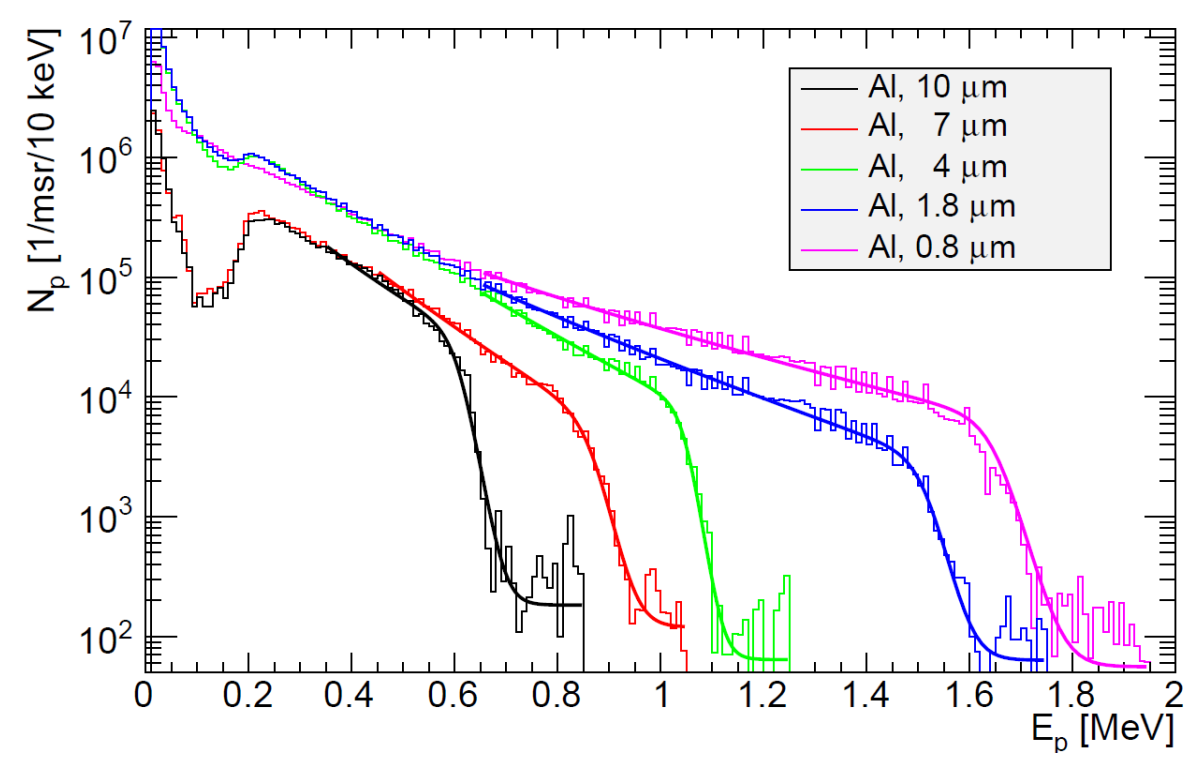

(b)

Figure R.4: (a) Espectro de protones para diferentes espesores de blancos de aluminio en la correspondiente a la campaña experimental de bajo contraste. (b) Espectro de protones para diferentes espesores de blancos de aluminio en la correspondiente a la campaña experimental de alto contraste. 


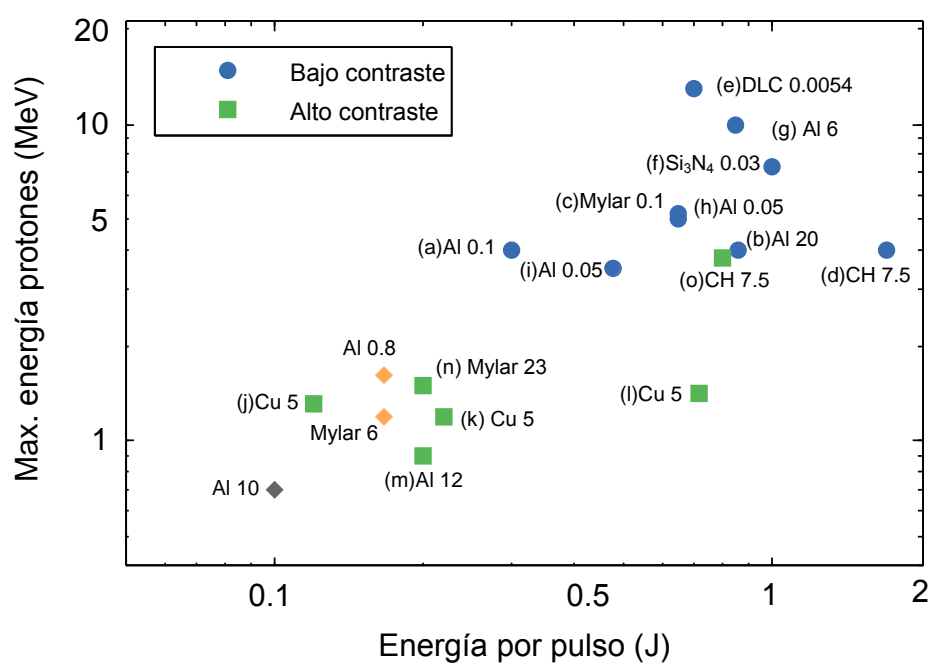

Figure R.5: Resultados experimentales comparados con la literatura. Los círculos azules representan experimentos usando láseres con contraste alto mientras que los cuadrados verdes son experimentos usando láseres con contraste bajo. Los puntos con forma de diamante representan los resultados obtenidos en esta tesis. Cada etiqueta exhibe la referencia bibliográfica y el material y espesor (en micras) de los blancos utilizados en cada experimento. (a) Neely et al. [82], (b) Kaluza et al. [40], (c) Ceccotti et al. [77], (d) Nishiuchi et al. [83], (e) Henig et al. [84],(f) Antici et al. [85], (g) Fritzler et al. [22], (h) A.P Robinson et al. [86], (i) J.S. Green et al. [87], (k) Fuiji et al. [81], (l) y (o) Yogo et al. [72, 80], (m) y (n) Spencer et al. [79].

En la segunda tanda, cuyos resultados puede observarse en la figura R.4(b), el láser disparaba con pulsos de casi $200 \mathrm{~mJ}$ de energía y 55 fs, una potencia pico de un poco menos de 4 TW y un contraste mayor de $10^{6}$.

En los experimentos realizados utilizando el láser con contraste bajo se obtuvo una energía máxima de protones de $0.7 \mathrm{MeV}$ para un blanco de aluminio de 18 $\mu \mathrm{m}$ de espesor. En cambio en los experimentos con alto contraste, se alcanzaron protones de $1.7 \mathrm{MeV}$ usando láminas de aluminio con espesores menores de $0.8 \mu \mathrm{m}$. Además, en la campaña realizada con bajo contraste no se observaron protones en ninguno de los disparos realizados sobre mylar, por lo que podemos concluir que las malas condiciones de contraste estaban provocando la aparición de pre-plasma que empeoró los resultados obtenidos.

En la figura R.5 se puede observar una comparativa de los resultados de los experimentos realizados durante esta tesis y algunos experimentos seleccionados de la literatura científica utilizando láseres de duración similar y energía por pulso 
menor a $1 \mathrm{~J}$.

\section{Conclusiones}

En esta tesis se ha presentado la construcción de un láser ultraintenso y ultracorto. La elección de Titanio:Zafiro como medio activo para desarrollar este láser se debe sobre todo a la madurez de la tecnología asociada y a que el zafiro tiene una conductividad térmica muy elevada por lo que es un material adecuado para soportar potencias elevadas.

Debido a que los bombeos actuales de Nd:YAG suelen usar lámparas de flash están limitados a $10 \mathrm{~Hz}$. Utilizando diodos láser se ha conseguido realizar un láser de varios TW de potencia pico y compacto, sin tener que utilizar refrigeración criogénica. Los bombeos se desarrollaron utilizando Nd:YAG y Nd:YLF con éxito, sobre todo este último material, capaz de generar mucha más energía que el de YAG. Dichos láseres de bombeo no fueron llevados a su máximo potencial pero se estima que puedan llegar a proporcionar hasta $1 \mathrm{~J}$ de energía cada uno en 527 nm. Con esta energía por pulso se podría llegar a generar un láser cerca de 10 TW funcionando a $100 \mathrm{~Hz}$.

Los experimentos de aceleración de protones fueron satisfactorios y encajan bien con otros resultados encontrados en literatura. El hecho de haber conseguido protones de $1.6 \mathrm{MeV}$ acelerados por un láser compacto y de alta potencia abre la puerta al desarrollo futuro de un blanco capaz de soportar la frecuencia de repetición del láser; de hecho los experimentos se realizaron en disparo único porque no contábamos con ningún tipo de soporte de varias láminas delgadas de aluminio que fuera los suficientemente rápido como para seguir al láser. Por último para mejorar el proceso de aceleración de protones, se están desarrollando blancos nanoestructurados que prometen mejorar la eficiencia del acoplamiento láser-blanco y así mejorar la energía máxima de los protones [89] 


\section{Bibliography}

[1] T. H. Maiman, "Stimulated optical radiation in ruby," nature 187, 493-494 (1960).

[2] H. W. Mocker and R. J. Collins, "Mode competition and self-locking effects in a q-switched ruby laser," Applied Physics Letters 7, 270-273 (1965).

[3] R. Fork, B. Greene, and C. V. Shank, "Generation of optical pulses shorter than 0.1 psec by colliding pulse mode locking," Applied Physics Letters 38, 671-672 (1981).

[4] D. E. Spence, P. N. Kean, and W. Sibbett, "60-fsec pulse generation from a self-mode-locked ti:sapphire laser," Opt. Lett. 16, 42-44 (1991).

[5] F. Böhle, M. Kretschmar, A. Jullien, M. Kovacs, M. Miranda, R. Romero, H. Crespo, U. Morgner, P. Simon, R. Lopez-Martens et al., "Compression of cep-stable multi-mj laser pulses down to $4 \mathrm{fs}$ in long hollow fibers," Laser Physics Letters 11, 095401 (2014).

[6] D. Strickland and G. Mourou, "Compression of amplified chirped optical pulses," Opt. Comm. 53, 219-221 (1985).

[7] T. Tajima, "Laser acceleration and its future," Proceedings of the Japan Academy, Series B 86, 147-157 (2010).

[8] W. P. Leemans, B. Nagler, A. J. Gonsalves, C. Toth, K. Nakamura, C. G. R. Geddes, E. Esarey, C. B. Schroeder, and S. M. Hooker, "Gev electron beams from a centimetre-scale accelerator," Nature Physics 6, 696 - 699 (2006).

[9] "Particle therapy co-operative group," (2017). 
[10] P. Chaudhary, T. I. Marshall, F. M. Perozziello, L. Manti, F. J. Currell, F. Hanton, S. J. McMahon, J. N. Kavanagh, G. A. P. Cirrone, F. Romano et al., "Relative biological effectiveness variation along monoenergetic and modulated bragg peaks of a 62-mev therapeutic proton beam: A preclinical assessment," International Journal of Radiation Oncology* Biology* Physics 90, 27-35 (2014).

[11] S. Houle, N. Ginovart, D. Hussey, J. Meyer, and A. Wilson, "Imaging the serotonin transporter with positron emission tomography: initial human studies with [11c] dapp and [11c] dasb," European journal of nuclear medicine 27, 1719-1722 (2000).

[12] R. A. Koeppe, S. Gilman, A. Joshi, S. Liu, R. Little, L. Junck, M. Heumann, K. A. Frey, and R. L. Albin, "11c-dtbz and 18f-fdg pet measures in differentiating dementias," Journal of Nuclear Medicine 46, 936-944 (2005).

[13] L. Farde, H. Hall, E. Ehrin, and G. Sedvall, "Quantitative analysis of d2 dopamine receptor binding in the living human brain by pet," Science 231, 258-261 (1986).

[14] M. M. Ter-Pogossian and P. Herscovitch, "Radioactive oxygen-15 in the study of cerebral blood flow, blood volume, and oxygen metabolism," Seminars in Nuclear Medicine 15, 377 - 394 (1985).

[15] M. G. Niemeyer, A. F. Kuijper, L. Gerhards, E. G. D'Haene, and E. E. van der Wall, "Nitrogen-13 ammonia perfusion imaging: Relation to metabolic imaging," American Heart Journal 125, 848 - 854 (1993).

[16] K. Schelstraete, M. Simons, J. Deman, F. L. Vermeulen, G. Siegers, C. Vandecasteele, P. Goethals, and A. D. Schryver, "Uptake of 13n-ammonia by human tumours as studied by positron emission tomography," The British Journal of Radiology 55, 797-804 (1982).

[17] P. Som, H. Atkins, D. Bandoypadhyay, J. Fowler, R. MacGregor, K. Matsui, Z. Oster, D. Sacker, C. Shiue, H. Turner et al., "A fluorinated glucose analog, 2-fluoro-2-deoxy-d-glucose (f-18): nontoxic tracer for rapid tumor detection," J Nucl Med 21, 670-675 (1980). 
[18] J. W. Fletcher, B. Djulbegovic, H. P. Soares, B. A. Siegel, V. J. Lowe, G. H. Lyman, R. E. Coleman, R. Wahl, J. C. Paschold, N. Avril et al., "Recommendations on the use of $18 \mathrm{f}-\mathrm{fdg}$ pet in oncology," Journal of Nuclear Medicine 49, 480-508 (2008).

[19] N. Otuka, E. Dupont, V. Semkova, B. Pritychenko, A. Blokhin, M. Aikawa, S. Babykina, M. Bossant, G. Chen, S. Dunaeva, R. Forrest, T. Fukahori, N. Furutachi, S. Ganesan, Z. Ge, O. Gritzay, M. Herman, S. Hlavač, K. Katō, B. Lalremruata, Y. Lee, A. Makinaga, K. Matsumoto, M. Mikhaylyukova, G. Pikulina, V. Pronyaev, A. Saxena, O. Schwerer, S. Simakov, N. Soppera, R. Suzuki, S. Takács, X. Tao, S. Taova, F. Tárkányi, V. Varlamov, J. Wang, S. Yang, V. Zerkin, and Y. Zhuang, "Towards a more complete and accurate experimental nuclear reaction data library (exfor): International collaboration between nuclear reaction data centres (nrdc)," Nuclear Data Sheets 120, 272 276 (2014).

[20] H. Daido, M. Nishiuchi, and A. S. Pirozhkov, "Review of laser-driven ion sources and their applications," Reports on Progress in Physics 75, 056401 (2012).

[21] I. Spencer, K. Ledingham, R. Singhal, T. McCanny, P. McKenna, E. Clark, K. Krushelnick, M. Zepf, F. Beg, M. Tatarakis, A. Dangor, P. Norreys, R. Clarke, R. Allott, and I. Ross, "Laser generation of proton beams for the production of short-lived positron emitting radioisotopes," Nuclear Instruments and Methods in Physics Research Section B: Beam Interactions with Materials and Atoms 183, 449 - 458 (2001).

[22] S. Fritzler, V. Malka, G. Grillon, J. P. Rousseau, F. Burgy, E. Lefebvre, E. d'Humières, P. McKenna, and K. W. D. Ledingham, "Proton beams generated with high-intensity lasers: Applications to medical isotope production," Applied Physics Letters 83, 3039-3041 (2003).

[23] K. W. D. Ledingham, P. McKenna, T. McCanny, S. Shimizu, J. M. Yang, L. Robson, J. Zweit, J. M. Gillies, J. Bailey, G. N. Chimon, R. J. Clarke, D. Neely, P. A. Norreys, J. L. Collier, R. P. Singhal, M. S. Wei, S. P. D. Mangles, 
P. Nilson, K. Krushelnick, and M. Zepf, "High power laser production of shortlived isotopes for positron emission tomography," Journal of Physics D: Applied Physics 37, 2341 (2004).

[24] E. Lefebvre, E. d'Humières, S. Fritzler, and V. Malka, "Numerical simulation of isotope production for positron emission tomography with laser-accelerated ions," Journal of applied physics 100, 113308 (2006).

[25] M. Seimetz, P. Bellido, F. Sánchez, R. Lera, A. R. d. la Cruz, S. Torres-Peiro, L. Roso, A. Aguilar, P. Conde, A. J. Gonzalez, A. Iborra, L. Moliner, J. P. Rigla, M. J. Rodríguez-Álvarez, S. Sanchez, A. Soriano, and J. M. Benlloch, "Detailed requirements for a laser-based proton/ion accelerator for radioisotope production," in "2015 IEEE Nuclear Science Symposium and Medical Imaging Conference (NSS/MIC)," (2015), pp. 1-5.

[26] Y. Kitagawa, H. Fujita, R. Kodama, H. Yoshida, S. Matsuo, T. Jitsuno, T. Kawasaki, H. Kitamura, T. Kanabe, S. Sakabe et al., "Prepulse-free petawatt laser for a fast ignitor," IEEE Journal of Quantum Electronics 40, 281-293 (2004).

[27] R. Keyes and T. Quist, "Injection luminescent pumping of caf2: U3+ with gaas diode lasers," Applied Physics Letters 4, 50-52 (1964).

[28] R. L. Byer, "Diode laser-pumped solid-state lasers," Science 239, 742-748 (1988).

[29] L. M. Frantz and J. S. Nodvik, "Theory of pulse propagation in a laser amplifier," Journal of Applied Physics 34, 2346-2349 (1963).

[30] O. Uteza, B. Bussiere, F. Canova, J.-P. Chambaret, P. Delaporte, T. Itina, and M. Sentis, "Laser-induced damage threshold of sapphire in nanosecond, picosecond and femtosecond regimes," Applied Surface Science 254, 799-803 (2007).

[31] J.-F. Bisson, Y. Feng, A. Shirakawa, H. Yoneda, J. Lu, H. Yagi, T. Yanagitani, and K.-I. Ueda, "Laser damage threshold of ceramic yag," Japanese journal of applied physics 42, L1025 (2003). 
[32] E. G. Gamaly, A. V. Rode, B. Luther-Davies, and V. T. Tikhonchuk, "Ablation of solids by femtosecond lasers: Ablation mechanism and ablation thresholds for metals and dielectrics," Physics of plasmas 9, 949-957 (2002).

[33] W. Lowdermilk and D. Milam, "Laser-induced surface and coating damage," IEEE Journal of Quantum Electronics 17, 1888-1903 (1981).

[34] H. Kogelnik, "On the propagation of gaussian beams of light through lenslike media including those with a loss or gain variation," Appl. Opt. 4, 1562-1569 (1965).

[35] L. W. Casperson, "Gaussian light beams in inhomogeneous media," Appl. Opt. 12, 2434-2441 (1973).

[36] C. Le Blanc, P. Curley, and F. Salin, "Gain-narrowing and gain-shifting of ultra-short pulses in ti: sapphire amplifiers," Optics Communications 131, 391-398 (1996).

[37] L. Waxer, V. Bagnoud, I. Begishev, M. Guardalben, J. Puth, and J. Zuegel, "High-conversion-efficiency optical parametric chirped-pulse amplification system using spatiotemporally shaped pump pulses," Optics letters 28, 1245-1247 (2003).

[38] R. Stoian, M. Boyle, A. Thoss, A. Rosenfeld, G. Korn, I. Hertel, and E. Campbell, "Laser ablation of dielectrics with temporally shaped femtosecond pulses," Applied physics letters 80, 353-355 (2002).

[39] S. Bulanov, A. Brantov, V. Y. Bychenkov, V. Chvykov, G. Kalinchenko, T. Matsuoka, P. Rousseau, S. Reed, V. Yanovsky, D. Litzenberg et al., "Accelerating monoenergetic protons from ultrathin foils by flat-top laser pulses in the directed-coulomb-explosion regime," Physical Review E 78, 026412 (2008).

[40] M. Kaluza, J. Schreiber, M. I. Santala, G. D. Tsakiris, K. Eidmann, J. Meyerter Vehn, and K. J. Witte, "Influence of the laser prepulse on proton acceleration in thin-foil experiments," Physical review letters 93, 045003 (2004). 
[41] J. E. Geusic, H. M. Marcos, and L. G. V. Uitert, "Laser oscillations in nddoped yttrium aluminum, yttrium gallium and gadolinium garnets," Applied Physics Letters 4, 182-184 (1964).

[42] H. G. Danielmeyer, M. Blätte, and P. Balmer, "Fluorescence quenching in nd:yag," Applied physics 1, 269-274 (1973).

[43] G. Zhang, H. Zhu, C. Huang, J. Chen, Y. Wei, and L. Huang, "Diode-sidepumped nd:yag laser at 1338 nm," Opt. Lett. 34, 1495-1497 (2009).

[44] S. G. Grechin and P. P. Nikolaev, "Diode-side-pumped laser heads for solidstate lasers," Quantum Electronics 39, 1 (2009).

[45] S. Thibault, "Astigmatic light source in optical design software," Optical Engineering 39, $39-39-4$ (2000).

[46] J. Arasa and J. Alda, Real Ray Tracing (Mark Dekker, 2004).

[47] W. Koechner, "Thermal lensing in a nd:yag laser rod," Appl. Opt. 9, 2548-2553 (1970).

[48] R. Lausten and P. Balling, "Thermal lensing in pulsed laser amplifiers: an analytical model," J. Opt. Soc. Am. B 20, 1479-1485 (2003).

[49] W. A. Clarkson, N. S. Felgate, and D. C. Hanna, "Simple method for reducing the depolarization loss resulting from thermally induced birefringence in solidstate lasers," Opt. Lett. 24, 820-822 (1999).

[50] D. Findlay and R. Clay, "The measurement of internal losses in 4-level lasers," Physics Letters 20, 277-278 (1966).

[51] J. J. Degnan, "Theory of the optimally coupled q-switched laser," IEEE Journal of Quantum Electronics 25, 214-220 (1989).

[52] H. Kogelnik and T. Li, "Laser beams and resonators," Appl. Opt. 5, 1550-1567 (1966).

[53] D. C. Hanna, C. G. Sawyers, and M. A. Yuratich, "Telescopic resonators for large-volume tem00-mode operation," Optical and Quantum Electronics 13, 493-507 (1981). 
[54] P. F. Moulton, "Spectroscopic and laser characteristics of ti:al2o3," J. Opt. Soc. Am. B 3, 125-133 (1986).

[55] R. Ell, U. Morgner, F. X. Kärtner, J. G. Fujimoto, E. P. Ippen, V. Scheuer, G. Angelow, T. Tschudi, M. J. Lederer, A. Boiko, and B. Luther-Davies, "Generation of 5-fs pulses and octave-spanning spectra directly from a ti:sapphire laser," Opt. Lett. 26, 373-375 (2001).

[56] D. E. McCumber, "Einstein relations connecting broadband emission and absorption spectra," Phys. Rev. 136, A954-A957 (1964).

[57] A. Hoffstädt, "High-average-power flash-lamp-pumped ti:sapphire laser," Opt. Lett. 19, 1523-1525 (1994).

[58] C. H. Muller, R. A. Hamil, G. C. Tisone, D. D. Lowenthal, and K. W. Kangas, “2.0-j ti:sapphire laser oscillator," Opt. Lett. 13, 380-382 (1988).

[59] P. W. Roth, A. J. Maclean, D. Burns, and A. J. Kemp, "Directly diode-laserpumped ti:sapphire laser," Opt. Lett. 34, 3334-3336 (2009).

[60] P. W. Roth, D. Burns, and A. J. Kemp, "Power scaling of a directly diodelaser-pumped ti:sapphire laser," Opt. Express 20, 20629-20634 (2012).

[61] S. Backus, C. G. D. III, M. M. Murnane, and H. C. Kapteyn, "High power ultrafast lasers," Review of Scientific Instruments 69, 1207-1223 (1998).

[62] G. Cheriaux, B. Walker, L. F. Dimauro, P. Rousseau, F. Salin, and J. P. Chambaret, "Aberration-free stretcher design for ultrashort-pulse amplification," Opt. Lett. 21, 414-416 (1996).

[63] P. Tournois, "Acousto-optic programmable dispersive filter for adaptive compensation of group delay time dispersion in laser systems," Opt. Comm. 140, $245-249$ (1997).

[64] P. A. Schulz and S. R. Henion, "Liquid-nitrogen-cooled ti: Al/sub 2/o/sub 3/laser," IEEE Journal of Quantum Electronics 27, 1039-1047 (1991). 
[65] J. P. Chambaret, C. L. Blanc, G. Chériaux, P. Curley, G. Darpentigny, P. Rousseau, G. Hamoniaux, A. Antonetti, and F. Salin, "Generation of 25-tw, 32-fs pulses at 10 hz," Opt. Lett. 21, 1921-1923 (1996).

[66] J. Zhou, C.-P. Huang, M. M. Murnane, and H. C. Kapteyn, "Amplification of 26-fs, 2-tw pulses near the gain-narrowing limit in ti:sapphire," Opt. Lett. 20, 64-66 (1995).

[67] A. Trisorio, S. Grabielle, M. Divall, N. Forget, and C. P. Hauri, "Self-referenced spectral interferometry for ultrashort infrared pulse characterization," Opt. Lett. 37, 2892-2894 (2012).

[68] E. Sarachik and G. Schappert, "Classical theory of the scattering of intense laser radiation by free electrons," Physical Review D 1, 2738 (1970).

[69] G. Malka, J. Fuchs, F. Amiranoff, S. Baton, R. Gaillard, J. Miquel, H. Pépin, C. Rousseaux, G. Bonnaud, M. Busquet et al., "Suprathermal electron generation and channel formation by an ultrarelativistic laser pulse in an underdense preformed plasma," Physical review letters 79, 2053 (1997).

[70] P. McKenna, K. W. D. Ledingham, I. Spencer, T. McCany, R. P. Singhal, C. Ziener, P. S. Foster, E. J. Divall, C. J. Hooker, D. Neely, A. J. Langley, R. J. Clarke, P. A. Norreys, K. Krushelnick, and E. L. Clark, "Characterization of multiterawatt laser-solid interactions for proton acceleration," Review of Scientific Instruments 73, 4176-4184 (2002).

[71] N. Sinenian, M. J. Rosenberg, M. Manuel, S. C. McDuffee, D. T. Casey, A. B. Zylstra, H. G. Rinderknecht, M. G. Johnson, F. H. Séguin, J. A. Frenje, C. K. Li, and R. D. Petrasso, "The response of cr-39 nuclear track detector to 1-9 mev protons," Review of Scientific Instruments 82, 103303 (2011).

[72] A. Yogo, H. Daido, A. Fukumi, Z. Li, K. Ogura, A. Sagisaka, A. S. Pirozhkov, S. Nakamura, Y. Iwashita, T. Shirai, A. Noda, Y. Oishi, T. Nayuki, T. Fujii, K. Nemoto, I. W. Choi, J. H. Sung, D.-K. Ko, J. Lee, M. Kaneda, and A. Itoh, "Laser prepulse dependency of proton-energy distributions in ultraintense laserfoil interactions with an online time-of-flight technique," Physics of Plasmas 14, 043104 (2007). 
[73] M. Seimetz, P. Bellido, A. Soriano, J. G. López, M. C. Jiménez-Ramos, B. Fernández, P. Conde, E. Crespo, A. J. González, L. Hernández, A. Iborra, L. Moliner, J. P. Rigla, M. J. Rodríguez-Álvarez, F. Sánchez, S. Sánchez, L. F. Vidal, and J. M. Benlloch, "Calibration and performance tests of detectors for laser-accelerated protons," IEEE Transactions on Nuclear Science 62, 3216-3224 (2015).

[74] P. Bellido, M. Seimetz, R. Lera, A. R. de la Cruz, S. Torres-Peiró, A. J. González, F. Sánchez, L. Roso, and J. M. Benlloch, "Design of a thomson parabola spectrometer for the detection of laser-accelerated protons and ions," in "2015 IEEE Nuclear Science Symposium and Medical Imaging Conference (NSS/MIC)," (2015), pp. 1-4.

[75] P. Bellido, R. Lera, M. Seimetz, A. R. de la Cruz, S. Torres-Peirò, M. Galán, P. Mur, I. Sánchez, R. Zaffino, L. Vidal, A. Soriano, S. Sánchez, F. Sánchez, M. Rodríguez-Álvarez, J. Rigla, L. Moliner, A. Iborra, L. Hernández, D. GrauRuiz, A. González, J. García-Garrigos, E. Díaz-Caballero, P. Conde, A. Aguilar, L. Roso, and J. Benlloch, "Characterization of protons accelerated from a 3 tw table-top laser system," Journal of Instrumentation 12, T05001 (2017).

[76] P. McKenna, F. Lindau, O. Lundh, D. Neely, A. Persson, and C.-G. Wahlström, "High-intensity laser-driven proton acceleration: Influence of pulse contrast," Philosophical Transactions: Mathematical, Physical and Engineering Sciences 364, 711-723 (2006).

[77] T. Ceccotti, A. Lévy, H. Popescu, F. Réau, P. D’Oliveira, P. Monot, J. P. Geindre, E. Lefebvre, and P. Martin, "Proton acceleration with high-intensity ultrahigh-contrast laser pulses," Phys. Rev. Lett. 99, 185002 (2007).

[78] Y. Oishi, T. Nayuki, T. Fujii, Y. Takizawa, X. Wang, T. Yamazaki, K. Nemoto, T. Kayoiji, T. Sekiya, K. Horioka, Y. Okano, Y. Hironaka, K. G. Nakamura, K. Kondo, and A. A. Andreev, "Dependence on laser intensity and pulse duration in proton acceleration by irradiation of ultrashort laser pulses on a cu foil target," Physics of Plasmas 12, 073102 (2005). 
[79] I. Spencer, K. W. D. Ledingham, P. McKenna, T. McCanny, R. P. Singhal, P. S. Foster, D. Neely, A. J. Langley, E. J. Divall, C. J. Hooker, R. J. Clarke, P. A. Norreys, E. L. Clark, K. Krushelnick, and J. R. Davies, "Experimental study of proton emission from 60-fs, 200-mj high-repetition-rate tabletop-laser pulses interacting with solid targets," Phys. Rev. E 67, 046402 (2003).

[80] A. Yogo, H. Daido, S. Bulanov, K. Nemoto, Y. Oishi, T. Nayuki, T. Fujii, K. Ogura, S. Orimo, A. Sagisaka et al., "Laser ion acceleration via control of the near-critical density target," Physical Review E 77, 016401 (2008).

[81] T. Fujii, Y. Oishi, T. Nayuki, Y. Takizawa, K. Nemoto, T. Kayoiji, K. Horioka, Y. Okano, Y. Hironaka, K. G. Nakamura, and K. ichi Kondo, "Mev-order proton and carbon ion acceleration by irradiation of $60 \mathrm{fs}$ tw laser pulses on thin copper tape," Applied Physics Letters 83, 1524-1526 (2003).

[82] D. Neely, P. Foster, A. Robinson, F. Lindau, O. Lundh, A. Persson, C.-G. Wahlström, and P. McKenna, "Enhanced proton beams from ultrathin targets driven by high contrast laser pulses," Applied Physics Letters 89, 021502 (2006).

[83] M. Nishiuchi, H. Daido, A. Yogo, S. Orimo, K. Ogura, J. Ma, A. Sagisaka, M. Mori, A. Pirozhkov, H. Kiriyama et al., "Efficient production of a collimated mev proton beam from a polyimide target driven by an intense femtosecond laser pulse," Physics of Plasmas 15, 053104 (2008).

[84] A. Henig, S. Steinke, M. Schnürer, T. Sokollik, R. Hörlein, D. Kiefer, D. Jung, J. Schreiber, B. Hegelich, X. Yan et al., "Radiation-pressure acceleration of ion beams driven by circularly polarized laser pulses," Physical Review Letters 103, 245003 (2009).

[85] P. Antici, J. Fuchs, E. d'Humières, E. Lefebvre, M. Borghesi, E. Brambrink, C. Cecchetti, S. Gaillard, L. Romagnani, Y. Sentoku et al., "Energetic protons generated by ultrahigh contrast laser pulses interacting with ultrathin targets," Physics of Plasmas 14, 030701 (2007).

[86] A. Robinson, P. Foster, D. Adams, D. Carroll, B. Dromey, S. Hawkes, S. Kar, Y. Li, K. Markey, P. Mckenna et al., "Spectral modification of laser-accelerated 
proton beams by self-generated magnetic fields," New Journal of Physics 11, 083018 (2009).

[87] J. Green, D. Carroll, C. Brenner, B. Dromey, P. Foster, S. Kar, Y. Li, K. Markey, P. McKenna, D. Neely et al., "Enhanced proton flux in the mev range by defocused laser irradiation," New Journal of Physics 12, 085012 (2010).

[88] K. Zeil, S. D. Kraft, S. Bock, M. Bussmann, T. E. Cowan, T. Kluge, J. Metzkes, T. Richter, R. Sauerbrey, and U. Schramm, "The scaling of proton energies in ultrashort pulse laser plasma acceleration," New Journal of Physics 12, 045015 (2010).

[89] A. Macchi, A. Sgattoni, S. Sinigardi, M. Borghesi, and M. Passoni, "Advanced strategies for ion acceleration using high-power lasers," Plasma Physics and Controlled Fusion 55, 124020 (2013). 